Prepared for the U.S. Department of Energy

Under Contract DE-AC05-76RL01830

\title{
Technical Basis for Scaling Relationships for the Pretreatment Engineering Platform
}

WL Kuhn DE Kurath

ST Arm SD Rassat

JL Huckaby

July 2008

\section{Pacific Northwest}

NATIONAL LABORATORY 


\title{
DISCLAIMER
}

This report was prepared as an account of work sponsored by an agency of the United States Government. Neither the United States Government nor any agency thereof, nor Battelle Memorial Institute, nor any of their employees, makes any warranty, express or implied, or assumes any legal liability or responsibility for the accuracy, completeness, or usefulness of any information, apparatus, product, or process disclosed, or represents that its use would not infringe privately owned rights. Reference herein to any specific commercial product, process, or service by trade name, trademark, manufacturer, or otherwise does not necessarily constitute or imply its endorsement, recommendation, or favoring by the United States Government or any agency thereof, or Battelle Memorial Institute. The views and opinions of authors expressed herein do not necessarily state or reflect those of the United States Government or any agency thereof.

\author{
PACIFIC NORTHWEST NATIONAL LABORATORY \\ operated by \\ BATTELLE \\ for the \\ UNITED STATES DEPARTMENT OF ENERGY \\ under Contract DE-AC05-76RL01830
}

Printed in the United States of America
Available to DOE and DOE contractors from the Office of Scientific and Technical Information,
P.O. Box 62, Oak Ridge, TN 37831-0062;
ph: (865) 576-8401
fax: $(865)$ 576-5728
email: reports@adonis.osti.gov

\begin{abstract}
Available to the public from the National Technical Information Service, U.S. Department of Commerce, 5285 Port Royal Rd., Springfield, VA 22161 ph: (800) 553-6847 fax: $(703) 605-6900$ email: orders@ntis.fedworld.gov online ordering: http://www.ntis.gov/ordering.htm
\end{abstract}

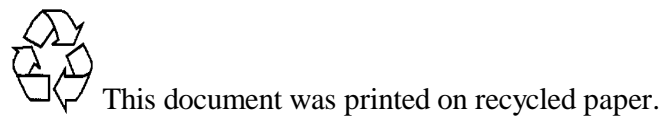


PNNL-16948

WTP-RPT-160, Rev 0

\title{
Technical Basis for Scaling Relationships for the Pretreatment Engineering Platform
}

\author{
W.L Kuhn \\ S.T. Arm \\ J.L. Huckaby \\ D.E. Kurath \\ S.D. Rassat
}

July 2008

Prepared for

the U.S. Department of Energy

under Contract DE-AC05-76RL01830

Pacific Northwest National Laboratory

Richland, Washington 99352 



\section{Executive Summary}

Pacific Northwest National Laboratory (PNNL) has been tasked by Bechtel National Inc. (BNI) on the River Protection Project-Waste Treatment Plant (RPP-WTP) project to perform research and development activities to resolve technical issues identified for the Pretreatment Facility (PTF). As part of this, the Pretreatment Engineering Platform (PEP) is designed and constructed as part of a plan to respond to issue M12, "Undemonstrated Leaching Processes," raised by the WTP External Flowsheet Review Team (EFRT). The PEP replicates the WTP leaching process using prototypic equipment and control strategies. The approach for scaling PEP performance data to predict WTP performance is critical to the successful resolution of the EFRT issue. This report describes the recommended PEP scaling approach.

\section{Results and Performance Against Objectives}

This report describes the scale-up methodology for PEP results as authorized through Subcontract Change Number SCN-26. The technical approach was a set of internal objectives that were satisfied as summarized in Table ES.1.

Table ES.1. Task Objective Evaluation

\begin{tabular}{|l|c|l||}
\hline \multicolumn{1}{|c|}{ Task Objective } & Objective Met & \multicolumn{1}{c|}{ Discussion } \\
\hline \hline $\begin{array}{l}\text { Review the rationale for the PEP } \\
\text { scale of 4.5. }\end{array}$ & Yes & $\begin{array}{l}\text { The rationale for the PEP scale of 4.5 was reviewed, } \\
\text { documented, and endorsed. }\end{array}$ \\
\hline $\begin{array}{l}\text { Define the technical basis for the } \\
\text { approach in scaling PEP } \\
\text { performance data to predict WTP } \\
\text { performance. }\end{array}$ & Yes & $\begin{array}{l}\text { The unit operations of the WTP replicated in the } \\
\text { PEP were analyzed to define scaling approaches } \\
\text { based on established engineering analysis } \\
\text { techniques. }\end{array}$ \\
\hline $\begin{array}{l}\text { Define how PEP performance data } \\
\text { will be used to predict WTP } \\
\text { performance. }\end{array}$ & Yes & $\begin{array}{l}\text { Anticipated PEP performance data were analyzed } \\
\text { and cross-referenced to the appropriate WTP } \\
\text { performance parameters. }\end{array}$ \\
\hline $\begin{array}{l}\text { Analyze the PEP system to identify } \\
\text { scaling issues and consider their } \\
\text { potential impacts and mitigation } \\
\text { approaches. }\end{array}$ & Yes & $\begin{array}{l}\text { The PEP design was considered in terms of scale-up } \\
\text { based on maintaining similitude. A number of } \\
\text { scaling issues were identified. A mitigating test } \\
\text { approach was recommended. }\end{array}$ \\
\hline $\begin{array}{l}\text { Provide input to test conduct and } \\
\text { data requirements. }\end{array}$ & Yes & $\begin{array}{l}\text { A test approach was recommended based on scaling } \\
\text { issues. }\end{array}$ \\
\hline
\end{tabular}

\section{Quality Requirements}

PNNL implements the RPP-WTP quality requirements by performing work in accordance with the River Protection Project - Waste Treatment Plant Support Program (RPP-WTP) Quality Assurance Plan (RPP-WTP-QA-001, QAP). Testing and analytical activities were performed to the quality requirements of NQA-1-1989 Part I, Basic and Supplementary Requirements, NQA-2a-1990, Part 2.7, and DOE/RW0333P, Rev 13, Quality Assurance Requirements and Descriptions. These quality requirements are implemented through the River Protection Project - Waste Treatment Plant Support Program (RPP-WTP) Quality Assurance Manual (RPP-WTP-QA-003, QAM). The analytical requirements are implemented through RPP-WTP's Statement of Work (RPP-WTP-QA-005) with the Radiochemical Processing Laboratory Analytical Service Operations. 
The RPP-WTP project addresses internal verification and validation activities by conducting an independent technical review of the final data report in accordance with RPP-WTP's procedure QA-RPPWTP-604. This review verifies that the reported results are traceable, that inferences and conclusions are soundly based, and the reported work satisfies the Test Plan objectives.

\section{Task Methodology}

The task described in this report essentially involved three activities:

1. A review of the applicable WTP unit operations and the PEP design and operational approach to achieve the desired M12 objectives. The PEP design was well-advanced when this task was initiated, so there was no opportunity to effect design changes. In particular, the scale factor of 4.5 (meaning the PEP is 4.5 linear times smaller than the WTP) was already established, but a review indicates its selection is sound. A review of the fundamental theory underpinning the applicable processes and physical and chemical phenomena (leaching and filtration and mixing and heat transfer) was undertaken, given the PEP unit operations. Given the fundamental theory, scaling assumptions and issues were identified and developed for resolution later in the task.

2. The fundamental theory was developed to formulate an approach for scaling PEP performance data to predict WTP behavior. Development was undertaken considering the specific mixing, leaching, heat transfer, filtration processes, and phenomena expected to occur in the PEP and WTP.

3. Given the scaling approach, an approach was developed for interpreting the anticipated PEP data for scaling up to predict WTP performance. A testing approach was also developed that mitigated the scaling issues identified in the first activity.

\section{Results Summary}

\section{Rationale for PEP Scale Factor of 4.5}

The basis of the PEP scale of 4.5 has been considered by the RPP-WTP project. Assessments of the techniques for scaling processes and evaluating the model data did not lead to a rationale for selecting the PEP scale. However, consideration of a number of factors led to the conclusion that the PEP scale factor should not exceed five. The intent to test full-scale filter membranes created the need to test at two processing rates to evaluate both the expected mixing and filter-flux behaviors. The simplest approach is to use a single filter assembly for testing WTP-scale processing rates and all five filters for PEP-scale process rates. Using a single 10-ft filter to represent the PEP-scale produces a scale factor of 4.58 . A scale factor 4.5 is obtained by including 12 filter membranes in the PEP filter bundle.

\section{PEP Scaling Issues}

Several scaling issues were identified. In general, the issues relate to inconsistencies between scaled mixing processes and various processes (filtration, washing, and leaching) that cannot practicably be scaled. For example, caustic and oxidative leach rates in the PEP will be equal to those expected in the WTP. However, the contents of the PEP vessels will be mixed faster because the vessels are smaller than in the WTP, and yet the air sparge velocity will be maintained and the pulse-jet mixer (PJM) frequency scaled to maintain the same turbulence characteristics. The impact of enhanced mixing could be assessed 
by conducting a test at reduced WTP PJM frequency. Similarly, filtration will occur at the same flux rate expected in the WTP. The effect of enhanced mixing can be assessed by employing more filter area to scale the flux rate.

\section{Technical Basis for the Approach in Scaling PEP Performance Data}

The foundations of comparing systems at different length and time scales are similitude and dimensional analysis. Similitude is the theory and art of predicting prototype performance from model observations. The art of similitude enters the problem when the engineer must make decisions about model design, model construction, performance of tests, or analysis of results that are not included in the basic theory. The principal use of dimensional analysis is to deduce, from a study of the dimensions of the variables in any physical system, certain limitations on any possible relationship between those variables.

Some of the key physical and chemical attributes affecting the performance of the pretreatment facility are summarized below.

- Characteristic Times. It is useful to define characteristic times for residence times associated with volumetric flow rates. The characteristic time is defined as the ratio of the characteristic length scale and the characteristic velocity imposed on the system. For cyclic processes, there are also interval times, such as the pulse interval and the pulse duration for PJMs. Concentrations change relative to the characteristic hydrodynamic time because the time constant for a mass balance transient mass is a residence time. The characteristic time to attain a specified chemical conversion depends on intensive variables (concentration, temperature, and particle-size distribution) and hence does not scale with the length scale of a system. On the other hand, the characteristic time to heat or cool a system depends on the mass of the system and hence on the length scale. Whether or not other phenomena (e.g., filtration, washing, or leaching) proceed in the PEP with more or less mixing than in the WTP is represented by the ratio of the characteristic mixing time to the characteristic time for that process. If the ratio is greater for the PEP than for the WTP, then for that process, PEP is "under mixed;" if the ratio is less, then the PEP is "over mixed."

- Hydrodynamics. Process slurries in the WTP tend to be either Newtonian fluids or non-Newtonian "Bingham plastics," which are fluids for which that the strain rate is proportional to the shear stress reduced by a "yield stress." In addition, stagnant Bingham plastic fluids can exhibit a shear strength greater than the yield stress that must be attained before any flow begins. The forces acting on fluid systems can be described as stresses categorized by an imposed characteristic velocity and a characteristic length together with the fluid properties of density, viscosity, yield stress, and interfacial tension. The ratios of these stresses form well-established dimensionless groups. Given the same solids, particle-size distribution, and liquid composition to provide representative solids for leaching, the shear strength will be the same for materials in the PEP as for materials in the WTP. Therefore, jet velocities (filter loop recirculation entrance, PJM orifice) are matched between the PEP and the WTP so that the shear strength Reynolds number is matched.

For systems undergoing "forced" convection, a characteristic velocity is imposed on the fluid. For systems undergoing "free" convection, no velocity is imposed as a boundary condition. Instead, a density difference is imposed through temperature or concentration differences, and the density difference causes flow through a force imbalance under the influence of gravity. Two characteristic 
terminal velocities are caused by density differences under the influence of gravity. A particle settles in Newtonian fluids in accordance with Stokes law. A bubble rises at a rate determined by the Froude number based on the size of the bubble, and the size of the bubble is based on the Bond number. Consequently, both the settling rate of particles and the rise velocity of bubbles scale with fluid properties; neither scales with the size of the system in which it is embedded. Therefore, jet velocities (filter loop recirculation entrance, PJM orifice) are matched between the PEP and the WTP so that the ratio of jet velocity to particle settling velocity also is matched.

The heat flux at the boundary of a liquid in motion is expressed in terms of a heat-transfer coefficient that depends on the fluid motion and the difference between the temperature at the boundary and the average temperature in the fluid. Heat-transfer coefficients are scaled in terms of the dimensionless Nusselt number, a function of either the Reynolds number for forced convection or the Grashof number for natural convection. The heat-transfer coefficient is proportional to the Nusselt number and inversely proportional to the length scale. Analogous relationships apply for mass transfer.

- Submerged Turbulent Jets. When fluid is injected through an orifice at the boundary of a system, the fluid penetrates the system volume as a jet that ultimately breaks up turbulently and mixes with the surrounding fluid thereby mixing that fluid. The inertial forces are opposed by the shear stresses. The more motive inertial forces prevail over dampening shear stresses, the greater the jet momentum agitates the fluid in the tank. Although we do not formally define the "intensity of mixing," it is greater for larger Reynolds numbers, and hence it will be greater near submerged jets in the WTP than in the PEP.

The extent of the jet compared to the length scale will be the same for systems that are geometrically similar, which is true for the PEP compared to the WTP. This is also true if the yield Reynolds number is matched between systems. Therefore, we must rely on similitude between the PEP and the WTP to match the existence, size, and shape of mixing cavities in the PEP and the WTP. To this end, one must match the shear strength Reynolds number between the PEP and the WTP. Given the same rheological properties of the slurry in the PEP and the WTP, one must match velocities at the origins of jets between the PEP and the WTP, including notably the filter loop recirculation jet and PJM jets.

- Heat Transfer. A scaling analysis of heat transfer associated with the scaled and full-scale process vessels may be reasonably divided into processes internal to the vessel and those from the vessel wall outwards. External heat transfer is further subdivided: natural convection over the outer surface of the vessel with radiative exchange between the vessel exterior and its surroundings; forced convection through vessel jackets; and heat exchange between the vessel and all piping attachments, either through conduction along the pipes or via the flux of energy into or out of the vessels by fluid convection. The heat flow across the tank boundaries scales roughly as the area of the tank boundary. Internally, the vessel contents will convectively exchange heat with its surroundings, the vessel walls and the gas phase above.

In addition, heat is added through the direct addition of steam. However, the characteristic temperature difference attending heat transfer between injected steam and vaporization into sparge gas should be insubstantial.

- Filtration. The filtrate flux depends on the pressure drop from the filter feed to the filtrate and the degree of clogging of the filter media by fines in the slurry, which depends on the concentration of 
fines in the slurry and the elapsed time of filtration. If a filter cake also forms on the filter media, the filtrate rate depends also on the solids concentration in the slurry and the cross-flow velocity, which acts to continuously strip off solids from the filter cake. If the ratio of initial solids (and also initial fines) mass in the slurry to the filter area is equal between the WTP and PEP systems, then in either system, one can expect the filter flux to be the same function of a time constant that depends on the solids mass to filter area. Then the functional form observed in one system applies to the other system.

The rate of change of loading of clogging fines or caking solids during filtration are functions of concentrations of fines and solids in the slurry and time. This requires that, ultimately, the loading depends on the time and the solids mass/filter area ratio.

- Leaching. During caustic or oxidative leaching, suspended solids will react with the surrounding liquid to form soluble species. The conversion from the solid to dissolved form depends on the attributes of individual particles, the initial concentration of solids (as particles) in the slurry, the surface area of the particles relative to the initial volume of particles, a kinetic constant, the concentration of the reactant, and the concentration of the dissolved reaction product in solution. In the case of imperfect but homogenous mixing, the temperature varies randomly about a spatial mean. Because the leach rate increases exponentially with temperature, the volume-averaged conversion is greater than the conversion evaluated at the volume-averaged temperature if the temperature variations are randomly distributed. Therefore, quantifying modest variations in temperature is not particularly important to predicting the conversion; however, quantifying errors in measuring the volume-averaged temperature is very important because this sets a lower bound on the expected conversion. The effect of the mass-transfer resistance near the surface of a particle is determined by the dependence of the leach rate, in the absence of any mass-transfer resistance, on reactant and product concentrations in solution; the diffusivity of the reactants and products; and the size of the particle. The importance of a mass to any transfer resistance can be inferred from a single dimensionless group that combines all these dependencies. However, the time constant is short enough that any mass-transfer effect will not be important to interpreting PEP leach data, irrespective of the leach rate occurring in the absence of the mass-transfer resistance. In addition, mixing effects on mass transfer between waste particles and the leach solution will not be important.

\section{Methodology for Scaling Performance Data}

Five primary unit operations are associated with the PEP: dewatering, which is essentially filtration; washing, which is essentially combined mixing and filtration; caustic leaching, which includes heating and cooling operations; oxidative leaching; and addition of leaching reagents. Mixing performance is affected mainly by the Shear Reynolds number, if the slurry is non-Newtonian, and by the power/volume if the slurry is Newtonian. Slurry washing is affected mainly by the ability to mix condensate uniformly with slurry in a tank. Caustic leach rates are too slow to be limited by mass transfer and mixing effects, and even if leach rates are fast, then mass transfer limited-leaching would be too fast for the mass transfer rate to prevent acceptable performance. Therefore, for the same slurry properties, the caustic and oxidative leach factors observed in the PEP will be the same in the WTP for the same temperature and reaction time. The evolution of the filtrate flux during dewatering measured in the PEP will be a prediction of the evolution in the WTP given the same slurry composition, particle size and density distribution since full-length filters are being tested. 
Departures from ideal behavior (practically, where the concentration in the effluent from a tank equals the volume-averaged concentration in the tank) in the PEP or the WTP will probably be small because the systems are conservatively designed to be well-mixed. However, even if true, this may not be obvious because any test of the data against this assumption inevitably involves both actual departures from ideal behavior and errors in measuring variables that describe departures from ideal behavior. Thus, these contributions to the error must be separated to evaluate the correctness of the presumed model. Therefore, statistical methods should be applied that provide estimates of the true values of measured variables while simultaneously imposing constraints representing the presumed ideal behavior.

\section{Recommendations for Test Conduct and Data Requirements}

It is recommended that a test matrix for the PEP be established in terms of permutations of characteristic times that are practical to adjust. To preserve similitude in the sense of the ratios of time scales of processes affecting mixing, all characteristic process times must be kept in the same ratio between the PEP and the WTP. However, we expect behavior to develop in the PEP 4.5 times faster (time scale 4.5 smaller) than in the WTP. This is important to interpreting any transient behavior, which includes notably leaching and the evolution of a steady-state filtrate flux for a given solids concentration. Because characteristic velocities - i.e., at the orifice of the filter loop recirculation re-entry and the jet at the PJM orifice - are matched between the PEP and the WTP, the characteristic hydrodynamic times must be proportional to the length scales. The length scale is less for the PEP; therefore, the "engineered" hydrodynamic times are less than for the WTP. The effect of departures from similitude can be investigated by varying these ratios in the PEP relative to the WTP. 


\section{Acronyms and Abbreviations}

$\begin{array}{ll}\text { BNI } & \text { Bechtel National Inc. } \\ \text { DOE } & \text { U.S. Department of Energy } \\ \text { EFRT } & \text { External Flowsheet Review Team } \\ \text { HLW } & \text { high-level waste } \\ \text { LAW } & \text { low-activity waste } \\ \text { PEP } & \text { Pretreatment Engineering Platform } \\ \text { PJM } & \text { pulse-jet mixer } \\ \text { PNNL } & \text { Pacific Northwest National Laboratory } \\ \text { PTF } & \text { Pretreatment Facility } \\ \text { ROB } & \text { regions of bubbles } \\ \text { RPP } & \text { River Protection Project } \\ \text { TMP } & \text { trans-membrane pressure } \\ \text { UFP } & \text { Ultra-Filtration Process } \\ \text { WTP } & \text { Waste Treatment and Immobilization Plant } \\ \text { ZOI } & \text { zone of influence }\end{array}$




\section{Nomenclature}

\begin{tabular}{|c|c|c|c|}
\hline Symbol & Meaning & Units & Notes \\
\hline$A$ & Area & Meter $^{2}$ & $\begin{array}{l}\text { Specific meanings } \\
\text { indicated by subscripts as } \\
\text { explained in text }\end{array}$ \\
\hline$\widetilde{A}(X)$ & $\begin{array}{l}\text { Particle area distribution } \\
\text { function }\end{array}$ & None & $\begin{array}{l}\text { Area of particles after } \\
\text { conversion X }\end{array}$ \\
\hline$a, b, c, d, \ldots$ & Exponents & None & $\begin{array}{l}\text { In dimensional analysis } \\
\text { (see Appendix A, } \\
\text { Section A.1.5) }\end{array}$ \\
\hline$C$ & Concentration & (kilogram or mole) $/$ meter $^{3}$ & $\begin{array}{l}\text { Specific meanings } \\
\text { indicated by subscripts as } \\
\text { explained in text }\end{array}$ \\
\hline$C_{D}$ & Particle drag coefficient & None & \\
\hline$C_{p}$ & Specific heat & Joule/kilogram/Kelvin & \\
\hline$C_{w}$ & Average mass fraction & None & $\begin{array}{l}\text { In solids suspension } \\
\text { correlations }\end{array}$ \\
\hline$D$ & $\begin{array}{l}\text { Diameter or } \\
\text { characteristic transverse } \\
\text { dimension }\end{array}$ & Meter & $\begin{array}{l}\text { In hydrodynamic } \\
\text { relationships }\end{array}$ \\
\hline$D$ & Diffusivity & Meter $^{2} /$ second & $\begin{array}{l}\text { In mass transfer } \\
\text { relationships }\end{array}$ \\
\hline$D_{u}$ & Dispersion coefficient & Meter $^{2} /$ second & \\
\hline E & Activation energy & Joule/mole & \\
\hline Eo & Eotvos number & & $\begin{array}{l}\text { Defined in Appendix A, } \\
\text { Eq. A. } 212\end{array}$ \\
\hline$F$ & Volumetric flowrate & Meter ${ }^{3} /$ second & $\begin{array}{l}\text { In mass balance } \\
\text { relationships; } \\
\text { specific meanings } \\
\text { indicated by subscripts as } \\
\text { explained in text }\end{array}$ \\
\hline$F$ & Force & Joule-meter/second ${ }^{2}$ & In force balances \\
\hline$f_{Q}$ & Diffusive flux vector & (Units of Q) $/$ meter $^{2} /$ second & \\
\hline$F r$ & Froude number & None & $\begin{array}{l}\text { Defined in Appendix A, } \\
\text { Eq. A.190 }\end{array}$ \\
\hline$g$ & $\begin{array}{l}\text { Magnitude of } \\
\text { gravitational } \\
\text { acceleration (or } \\
\text { acceleration vector, if } \\
\text { shown in bold) }\end{array}$ & Meter/second ${ }^{2}$ & \\
\hline
\end{tabular}




\begin{tabular}{|c|c|c|c|}
\hline Symbol & Meaning & Units & Notes \\
\hline$G r$ & Grashof number & None & $\begin{array}{l}\text { Defined in Appendix A, } \\
\text { Eq. A.206 }\end{array}$ \\
\hline$h$ & Heat transfer coefficient & Watts/meter ${ }^{2} /$ Kelvin & $\begin{array}{l}\text { Specific meanings } \\
\text { indicated by subscripts as } \\
\text { explained in text }\end{array}$ \\
\hline$\Delta H^{\text {vap }}$ & $\begin{array}{l}\text { Enthalpy/mass of } \\
\text { vaporization }\end{array}$ & Joule/kilogram & \\
\hline$j$ & Mass flux & Kilogram $/$ meter $^{2} /$ second & \\
\hline$J_{0}$ & $\begin{array}{l}\text { rate of momentum } \\
\text { transport }\end{array}$ & Kilogram-meter/second ${ }^{2}$ & \\
\hline$k$ & Thermal conductivity & Watts/meter/Kelvin & $\begin{array}{l}\text { In heat transfer } \\
\text { relationships }\end{array}$ \\
\hline$k$ & Kinetic constant & Meter/second & \\
\hline$k_{C}$ & $\begin{array}{l}\text { Generalized mass } \\
\text { transfer resistance }\end{array}$ & Meter/second & $\begin{array}{l}\text { Defined implicitly in } \\
\text { Appendix A, Eq. A.97 }\end{array}$ \\
\hline$k_{m}$ & $\begin{array}{l}\text { Effective mass transfer } \\
\text { resistance }\end{array}$ & Meter/second & $\begin{array}{l}\text { Defined in Appendix A, } \\
\text { Eq. A. } 95\end{array}$ \\
\hline$k_{p}, k_{r}$ & $\begin{array}{l}\text { Mass transfer } \\
\text { coefficients for near- } \\
\text { particle diffusion of } \\
\text { reaction products ("p") } \\
\text { and reactants ("r") }\end{array}$ & meter/second & $\begin{array}{l}\text { Defined implicitly in } \\
\text { Appendix A, Eq. A.94 }\end{array}$ \\
\hline$L$ & Characteristic length & Meters & $\begin{array}{l}\text { Specific meanings } \\
\text { indicated by subscripts as } \\
\text { explained in text }\end{array}$ \\
\hline$m$ & Mass & Kilogram & $\begin{array}{l}\text { Specific meanings } \\
\text { indicated by subscripts as } \\
\text { explained in text }\end{array}$ \\
\hline$\dot{m}$ & Mass flow rate & Kilogram/second & \\
\hline$M$ & Molecular weight & Kilogram/mole & \\
\hline$M, L, t$ & Mass, length, time & Kilogram, meter, second & $\begin{array}{l}\text { In dimensional analysis } \\
\text { (see Appendix A, } \\
\text { Section A.1.5) }\end{array}$ \\
\hline$n$ & Exponent & None & \\
\hline$n \dot{n}$ & Molar flow rate & Moles/second & \\
\hline$N u$ & $\begin{array}{l}\text { Nusselt number, a } \\
\text { dimensionless heat } \\
\text { transfer } \\
\text { coefficient }=h L / k\end{array}$ & None & $\begin{array}{l}\text { In heat transfer } \\
\text { correlations }\end{array}$ \\
\hline$p, P$ & Pressure & Pascal & \\
\hline
\end{tabular}




\begin{tabular}{|c|c|c|c|}
\hline Symbol & Meaning & Units & Notes \\
\hline$P_{\text {sat }}$ & $\begin{array}{l}\text { Vapor pressure of } \\
\text { saturated liquid }\end{array}$ & Pascal & \\
\hline$p_{z}$ & Dynamic pressure & Pascal & See Appendix A, Eq. A.5 \\
\hline$Q$ & Rate of heat transfer & Watts & \\
\hline$q$ & Heat flux & Watt/meter ${ }^{2}$ & \\
\hline$Q(\boldsymbol{x}, t)$ & $\begin{array}{l}\text { General conserved } \\
\text { quantity, function of } \\
\text { vector position } \mathbf{x} \text { and } \\
\text { time } t\end{array}$ & Depends on the quantity & \\
\hline$r$ & Particle radius & Meter & $\begin{array}{l}\text { In particle dissolution } \\
\text { relationships }\end{array}$ \\
\hline$R$ & Gas constant & Joule/mole/Kelvin & \\
\hline$\widetilde{r}(X)$ & $\begin{array}{l}\text { Dimensionless reaction } \\
\text { rate function }\end{array}$ & None & $\begin{array}{l}\text { Rate of reaction after } \\
\text { conversion } X \text {, compared } \\
\text { to initial rate }\end{array}$ \\
\hline $\operatorname{Re}$ & Reynolds number & & $\begin{array}{l}\text { Specific meanings } \\
\text { indicated by subscripts as } \\
\text { explained in text; but has } \\
\text { general form } u L / v\end{array}$ \\
\hline$t$ & Time & Seconds & $\begin{array}{l}\text { Specific meanings } \\
\text { indicated by subscripts as } \\
\text { explained in text }\end{array}$ \\
\hline$T$ & Temperature & Kelvin & $\begin{array}{l}\text { Specific meanings } \\
\text { indicated by subscripts as } \\
\text { explained in text }\end{array}$ \\
\hline$u$ & Velocity & Meters/second & $\begin{array}{l}\text { Specific meanings } \\
\text { indicated by subscripts as } \\
\text { explained in text }\end{array}$ \\
\hline$\tilde{U}$ & Internal energy per mass & Joules/kilogram & \\
\hline$u_{F}$ & $\begin{array}{l}\text { Filter flux expressed as } \\
\text { velocity of liquid phase } \\
\text { normal to filter media }\end{array}$ & Meter/second & \\
\hline$V$ & Volume & Meter $^{3}$ & $\begin{array}{l}\text { Specific meanings } \\
\text { indicated by subscripts as } \\
\text { explained in text }\end{array}$ \\
\hline$w_{i}$ & $\begin{array}{l}\text { Mass fraction of } \\
\text { component ' } i \text { ' }\end{array}$ & None & \\
\hline$X$ & $\begin{array}{l}\text { Fractional chemical } \\
\text { conversion }\end{array}$ & None & \\
\hline$x$ & $\begin{array}{l}\text { General position } \\
\text { coordinate }\end{array}$ & Meters & \\
\hline
\end{tabular}




\begin{tabular}{|c|c|c|c|}
\hline Symbol & Meaning & Units & Notes \\
\hline$y$ & $\begin{array}{l}\text { Coordinate transverse to } \\
\text { flow }\end{array}$ & Meter & $\begin{array}{l}\text { In hydrodynamic } \\
\text { relationships }\end{array}$ \\
\hline$y_{i}$ & Mole fraction of "i" & None & \\
\hline$z$ & Upward coordinate & Meter & Hydrostatic relationships \\
\hline$z$ & Stream-wise coordinate & Meter & $\begin{array}{l}\text { Hydrodynamic } \\
\text { Relationships }\end{array}$ \\
\hline$\alpha$ & $\begin{array}{l}\text { Fraction of slurry } \\
\text { volume that is unmixed }\end{array}$ & None & $\begin{array}{l}\text { In mass balance } \\
\text { relationships }\end{array}$ \\
\hline$\alpha$ & $\begin{array}{l}\text { Relative mass transfer } \\
\text { resistance }\end{array}$ & None & $\begin{array}{l}\text { Defined in Appendix A, } \\
\text { Eq. A. } 96\end{array}$ \\
\hline$\alpha, \beta, \gamma, \delta$ & constants & None & $\begin{array}{l}\text { In dimensional analysis } \\
\text { (see Appendix A, } \\
\text { Section A.1.5) }\end{array}$ \\
\hline$\beta$ & $\begin{array}{l}\text { Fraction of slurry } \\
\text { volume consisting of } \\
\text { settled particles }\end{array}$ & None & \\
\hline$\beta_{T}, \beta_{C}$ & $\begin{array}{l}\text { Normalized } \\
\text { (temperature, } \\
\text { concentration) } \\
\text { dependence of density }\end{array}$ & & $\begin{array}{l}\text { Defined in Appendix A, } \\
\text { Eqns. A. } 203 \text { and A. } 206\end{array}$ \\
\hline$\Gamma$ & $\begin{array}{l}\text { Signifies Boundary for } \\
\text { flux boundary } \\
\text { conditions }\end{array}$ & None & \\
\hline$\delta$ & $\begin{array}{l}\text { Thicknesses associated } \\
\text { with fouling or filter } \\
\text { cake formation }\end{array}$ & meter & $\begin{array}{l}\text { Defined for specific } \\
\text { subscripts in conceptual } \\
\text { model for evolution of } \\
\text { filter flux }\end{array}$ \\
\hline$\Delta$ & $\begin{array}{l}\text { Rate of deformation of a } \\
\text { fluid }\end{array}$ & $1 /$ second & $\begin{array}{l}\text { Applies only in stress- } \\
\text { strain relationships }\end{array}$ \\
\hline$\varepsilon$ & Void fraction & None & $\begin{array}{l}\text { In hydrodynamic } \\
\text { relationships }\end{array}$ \\
\hline$\varepsilon$ & $\begin{array}{l}\text { Kinetic energy } \\
\text { dissipation per mass }\end{array}$ & Meter $^{2} /$ second $^{3}$ & $\begin{array}{l}\text { In hydrodynamic } \\
\text { relationships }\end{array}$ \\
\hline$\varepsilon_{X}$ & $\begin{array}{l}\text { Fraction of solids } \\
\text { approaching filter media } \\
\text { that are captured as } \\
\text { filter cake }\end{array}$ & None & $\begin{array}{l}\text { In filter flux conceptual } \\
\text { model }\end{array}$ \\
\hline$\Theta_{i}$ & $\begin{array}{l}\text { second derivative of } \\
\text { chemical conversion } \\
\text { with respect to } \\
\text { independent variable "i" }\end{array}$ & & \\
\hline
\end{tabular}




\begin{tabular}{|c|c|c|c|}
\hline Symbol & Meaning & Units & Notes \\
\hline$\lambda$ & $\begin{array}{l}\text { Ratio of heat added to } \\
\text { heat lost }\end{array}$ & None & Defined in Eq. 3.6 \\
\hline$\mu$ & Viscosity & Pascal-second & \\
\hline$\rho$ & Density & Kilogram/meter ${ }^{3}$ & $\begin{array}{l}\text { Specific meanings } \\
\text { indicated by subscripts as } \\
\text { explained in text }\end{array}$ \\
\hline$\sigma$ & Total stress & Pascal & \\
\hline$\tau$ & Dimensionless time & None & In transient analyses \\
\hline$\tau$ & Shear stress & Pascal & In hydrodynamic analyses \\
\hline$\tau_{0}$ & Yield shear stress & & \\
\hline$v$ & $\begin{array}{l}\text { Kinematic viscosity } \\
=\mu / \rho\end{array}$ & Meter $^{2} /$ second & $\begin{array}{l}\text { In hydrodynamic } \\
\text { relationships }\end{array}$ \\
\hline$v$ & $\begin{array}{l}\text { Mass of reaction } \\
\text { product produced per } \\
\text { mass of solid reaction }\end{array}$ & None & $\begin{array}{l}\text { In chemical conversion } \\
\text { relationships }\end{array}$ \\
\hline$\varphi$ & Volume fraction & None & \\
\hline$\varphi_{S 0}$ & $\begin{array}{l}\text { Volume fraction of } \\
\text { solids at start of } \\
\text { chemical conversion }\end{array}$ & None & \\
\hline$\chi$ & $\begin{array}{l}\text { Concentration or density } \\
\text { ratio }\end{array}$ & None & \\
\hline$\psi$ & $\begin{array}{l}\text { Concentrating volume } \\
\text { ratio }\end{array}$ & & \\
\hline$\omega$ & Wash factor & None & \\
\hline$\sim$ & $\begin{array}{l}\text { Above a symbol, } \\
\text { signifies the variable } \\
\text { divided by a } \\
\text { characteristic value }\end{array}$ & None & $\begin{array}{l}\text { See Appendix A, } \\
\text { Section A.1.4.1 }\end{array}$ \\
\hline$\nabla$ & Gradient operator & $1 /$ meter & \\
\hline
\end{tabular}




\section{Contents}

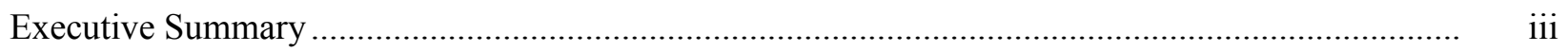

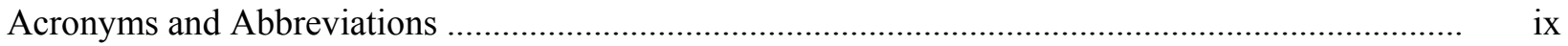

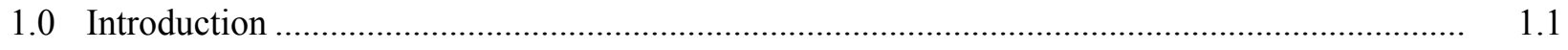

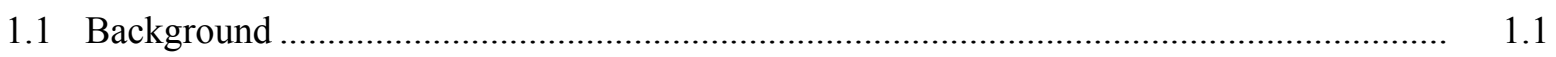

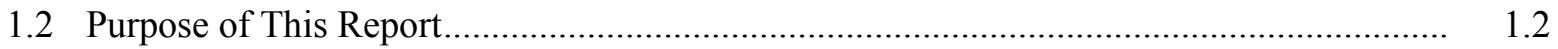

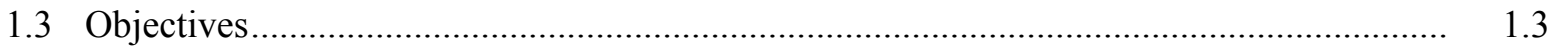

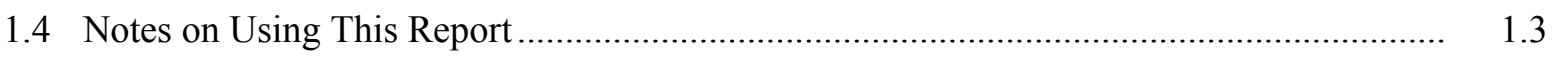

2.0 Technical Bases for Predicting the Performance of the PTF ................................................ 2.1

2.1 Predicting the Performance of the PTF From Similitude ................................................. 2.2

2.2 Predicting the Performance of the PTF From Design ....................................................... 2.3

3.0 Phenomena Controlling the Performance of the PTF .......................................................... 3.1

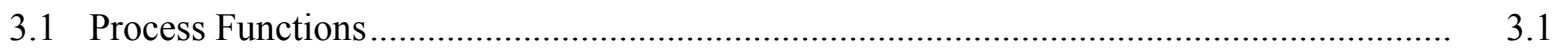

3.2 Considerations of Scale Common to All Process Steps ................................................. 3.1

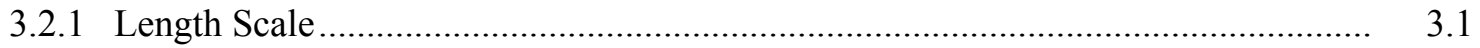

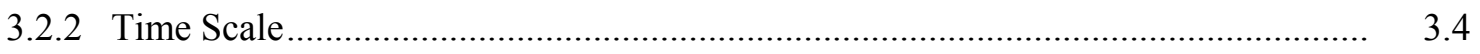

3.2.3 Thermal and Chemical "Scale" .................................................................... 3.5

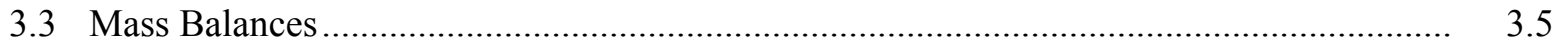

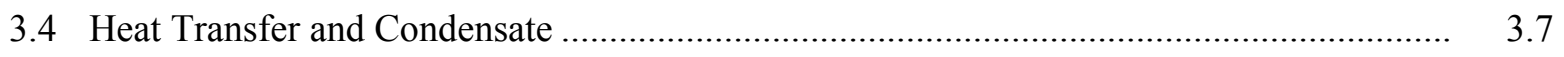

3.4.1 Effect of Size on Dilution by Condensation.................................................... 3.8

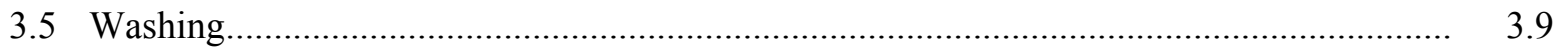

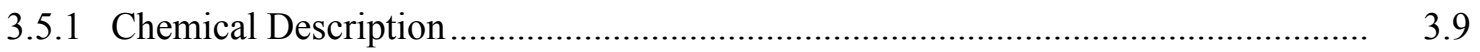

3.5.2 Design Basis Wash Factors ...................................................................... 3.10

3.5.3 Effects of Settled or Stagnant Regions ............................................................... 3.10

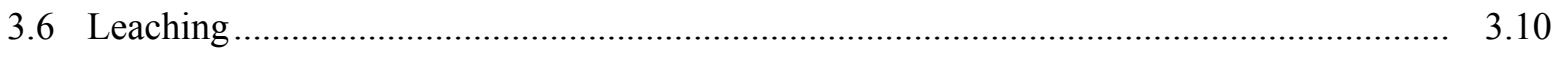

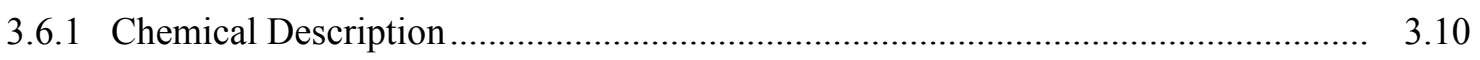

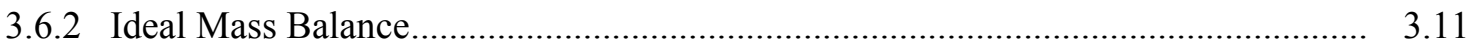

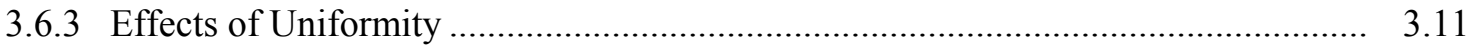

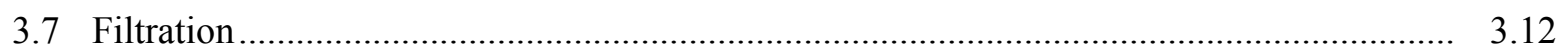

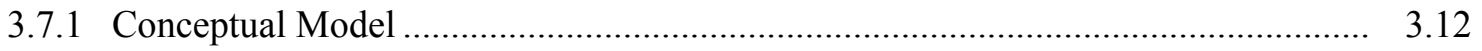

3.7.2 Complexity of Constituent Phenomena............................................................. 3.13

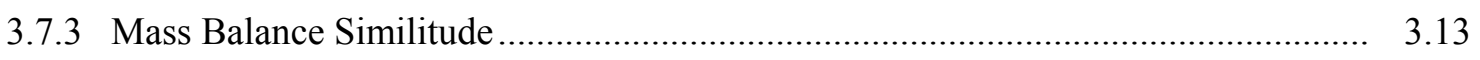

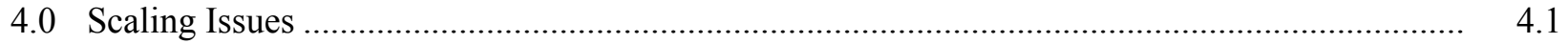

4.1 Limits on Application of Principles of Similitude ….................................................. 4.1

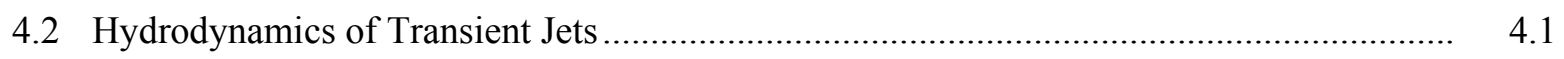

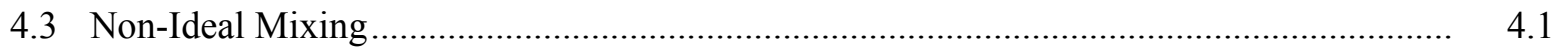

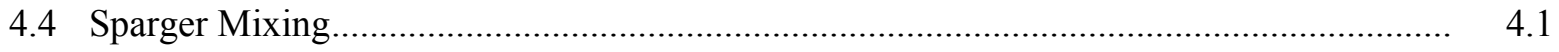




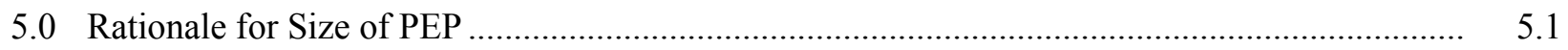

5.1 Practical Considerations Determining the Approximate Size ......................................... 5.1

5.2 Rationale for Exact Size of PEP ............................................................................ 5.2

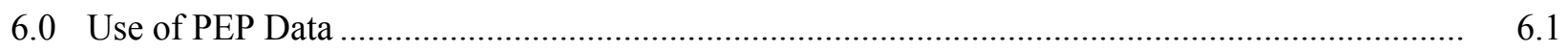

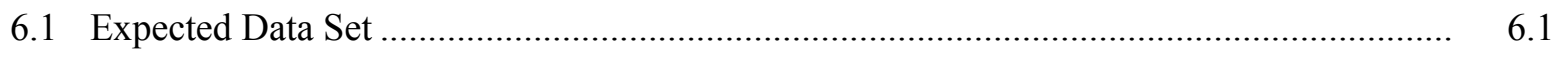

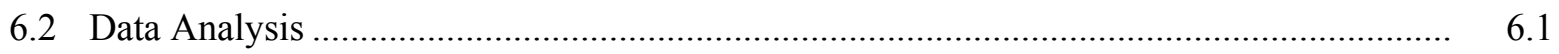

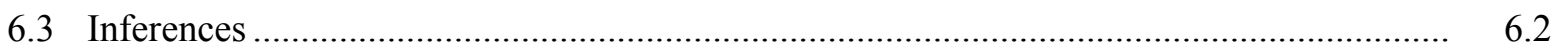

6.4 Correcting Mass Balances .................................................................................... 6.2

7.0 Technical Guidance for PEP Test Planning....................................................................

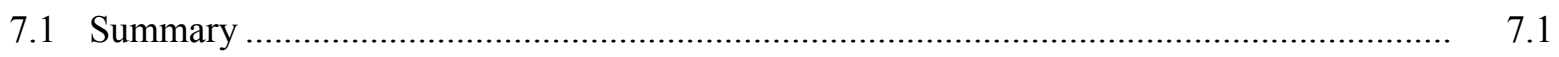

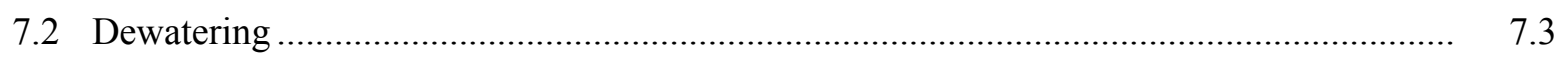

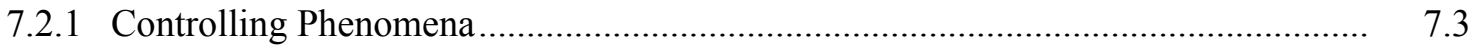

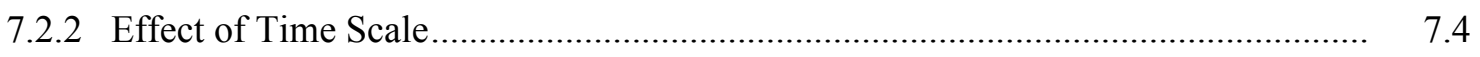

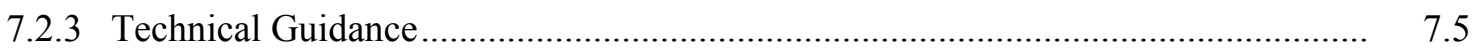

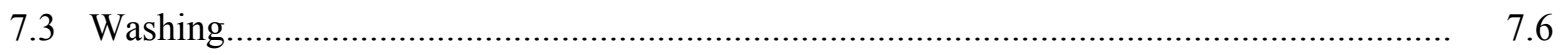

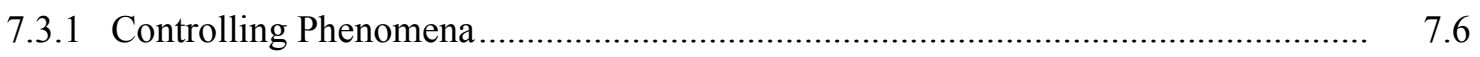

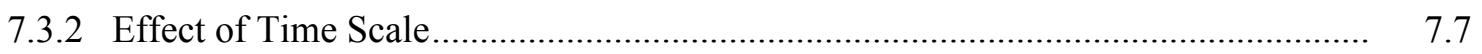

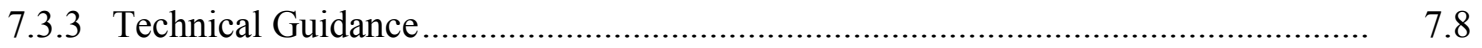

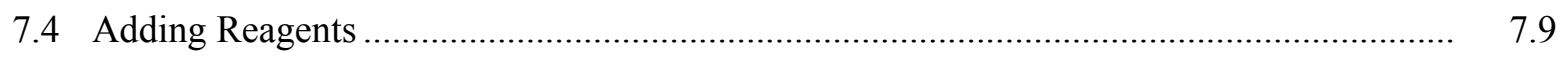

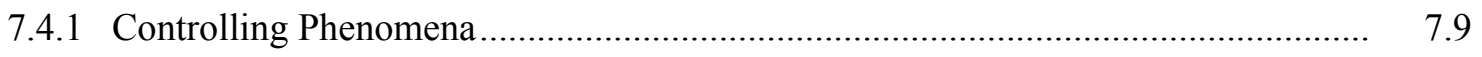

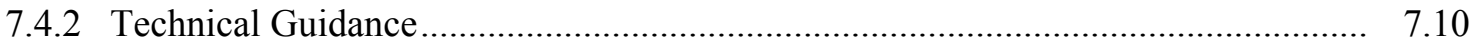

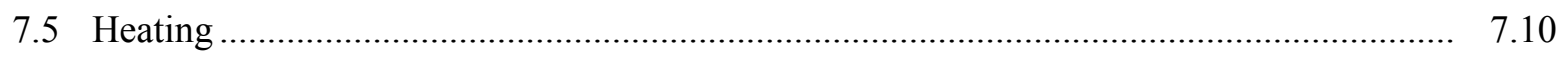

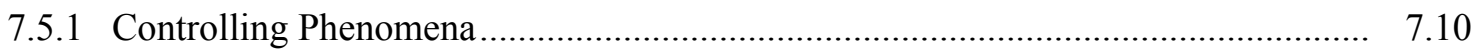

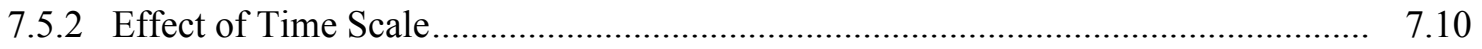

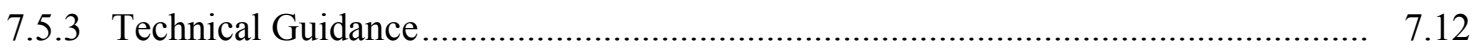

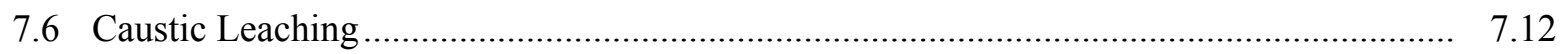

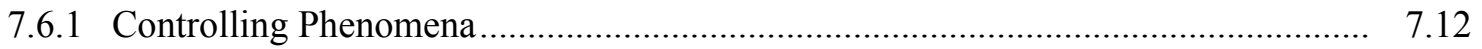

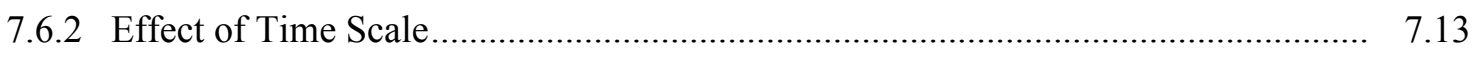

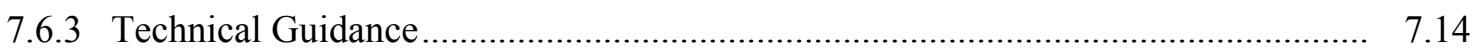

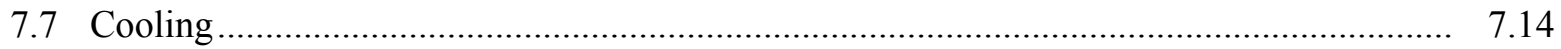

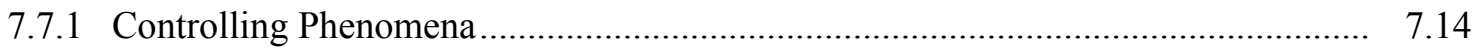

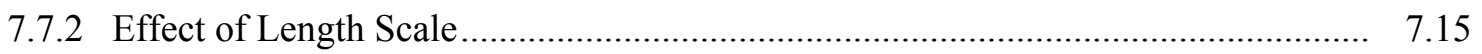

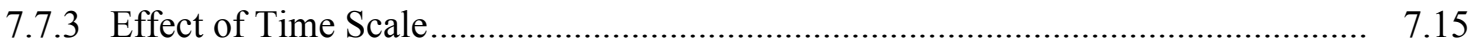

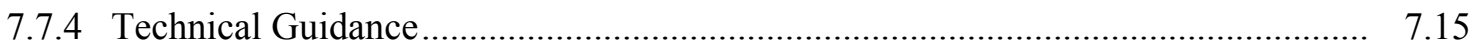

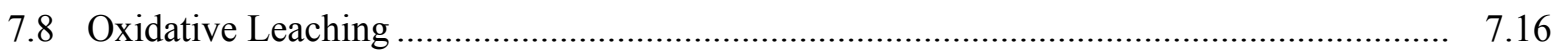

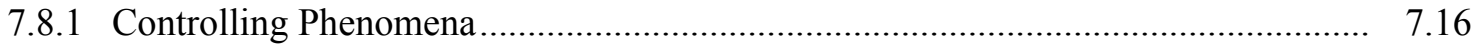

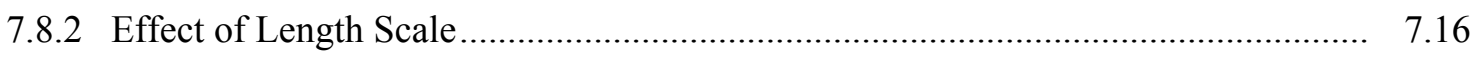

7.8.3 Effect of Time Scale ......................................................................................... 7.16

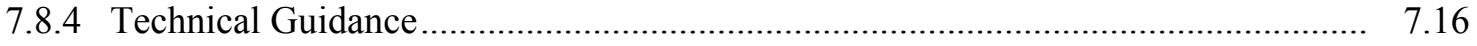




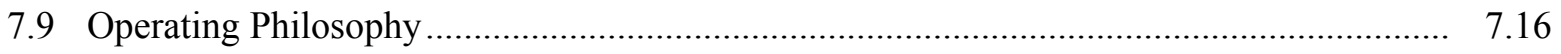

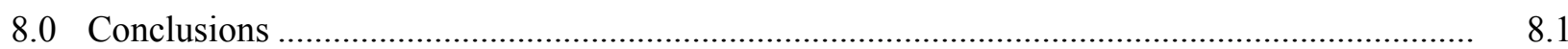

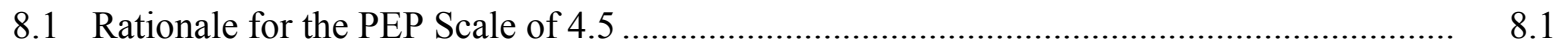

8.2 Technical Basis for the Approach in Scaling PEP Performance Data to Predict

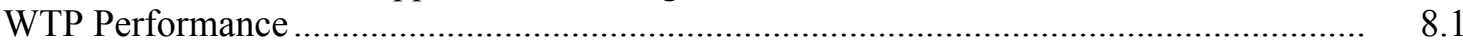

8.3 Use of PEP Performance Data to Predict WTP Performance ............................................ 8.1

8.4 Scaling Issues of PEP Design, Their Potential Impacts, and Mitigation Approaches.......... $\quad 8.1$ Appendix A - Process Details and Physico-Chemical Phenomena .................................................. A.1 Appendix B - Scaling Sparger Operations ….................................................................... B. 1

\section{Figures}

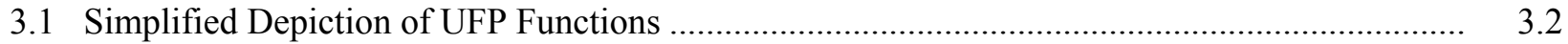

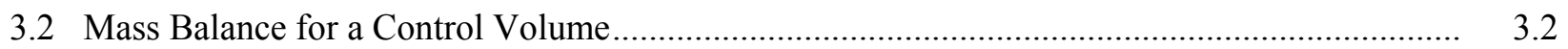

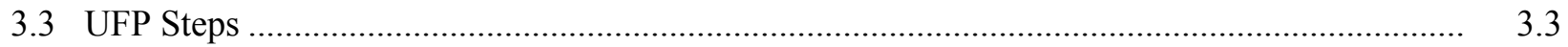

3.4 Filtration Hydrodynamic Environment ............................................................................. 3.12

\section{Tables}

ES.1 Task Objective Evaluation.......................................................................................... iii

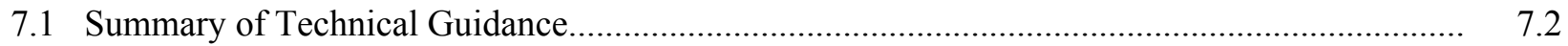





\subsection{Introduction}

This report provides the technical basis for scaling relationships for the Pretreatment Engineering Platform (PEP). "Scaling relationship" denotes a relationship of the behavior of one system to another with similar physical form with similar functions but built at a different length scale (usually smaller) and operated at a different time scale (usually faster). This is done to enable understanding of the process more economically, safely, and faster than by building and testing the system of ultimate interest.

\subsection{Background}

Pacific Northwest National Laboratory (PNNL) was tasked by Bechtel National Inc. (BNI) on the River Protection Project-Waste Treatment Plant (RPP-WTP) project to perform research and development activities. The purpose of the RPP-WTP project is to design, construct, and commission a plant to treat and immobilize high-level waste (HLW) and low-activity waste (LAW) stored in underground storage tanks at the Hanford Site.

In October 2005, an External Flowsheet Review Team (EFRT) was assembled with the charter to challenge and provide a critical review of the design of the Hanford Tank Waste Treatment and Immobilization Plant (WTP) through a thorough and critical review. The review ${ }^{1}$ identified a number of issues and potential issues that, when resolved, will significantly improve operation of the plant. One issue that must be addressed in the Pretreatment Facility (PTF) is number M12, "Undemonstrated Leaching Processes." The EFRT reported that neither the caustic-leaching nor the oxidative-leaching processes has been demonstrated at greater than bench scale. The EFRT argued that without a scale-up study, the ability to predict the effectiveness of these processes is limited.

The RPP-WTP project with the U.S. Department of Energy (DOE) developed an Issue Response Plan $^{2}$ to resolve the "Undemonstrated Leaching Processes" issue. The plan also addresses related topics that are not specifically in response to EFRT concerns. These include caustic addition and leaching issues that were revealed since the EFRT report was issued, information to support revision of the contract design basis for the PTF including system capacities, and earlier initiatives on enhancing plant throughput capacity. The selected solution for closure of these issues is to conduct scaled testing of the leaching (caustic and oxidative), washing processes, and filtration scenarios.

On October 23 and 24, 2006, the RPP-WTP Project "Ultrafiltration Leaching Process Technical Review Meeting" was held in Richland, Washington to review the M12 Response Plan. Meeting

\footnotetext{
${ }^{1}$ Letter: JP Henschel to RJ Schepens. March 17, 2006. Subject: Contract No. DE-AC27-01RV14136 - Report of External Flowsheet Review Team for the Hanford Tank Waste Treatment and Immobilization Plant - Final Report Titled: Comprehensive Review of the Waste Treatment Plant Flowsheet and Throughput, CCN: 132846, Bechtel National, Inc., Richland, Washington. (Attachment: Comprehensive Review of the Hanford Waste Treatment Plant Flowsheet and Throughput.)

${ }^{2}$ Barnes SM and R Voke. 2006. Issue Response Plan for Implementation of External Flowsheet Review Team (EFRT)Recommendations - M12, Undemonstrated Leaching Processes, 24590-WTP-PL-ENG-06-0024, Rev. 0, Bechtel National, Inc., Richland, Washington.
} 
participants included members of DOE, EFRT, the RPP-WTP project, and Battelle. ${ }^{1}$ As part of the review, some recommendations were made on the methodology and scale for performing the engineeringscale demonstration of the leaching processes. However, specific recommendations used in this report are referenced in Section 5.

Design and construction of the PEP has been undertaken by BNI and subcontractors to meet several general system requirements. The design includes principal processing equipment elements of the UltraFiltration Process (UFP) system, including UFP-VSL-00001 A/B, UFP-VSL-00002, the filter loop, recirculation pumps, and the ultrafilters with supporting equipment. Geometric similarity of the key pieces of processing equipment (including UFP-VSL-00001 A/B and UFP-VSL-00002) is being maintained. The ultrafilter assemblies with a prototypic length and tube diameter are scaled down by using fewer filter elements. Process monitoring instrumentation similar to that of the WTP has also been incorporated in the PEP design so it may be operated and controlled similarly to the WTP. The intent is for the PEP to perform in a similar manner to the $\mathrm{WTP}^{2}$, demonstrating aspects of the processes that meet expectations and helping to identify potential problems in the WTP.

The results from the PEP tests will provide input to improve predictions of WTP performance. Improved performance predictions will be based on actual waste and Phase 1 simulated waste in laboratory testing (i.e., characterization and parametric behavior testing), on Phase 1 simulated waste testing in the PEP, and on scaling analyses that relate the behaviors of the PEP and WTP processes. The PEP test results will be correlated with laboratory Phase 1 simulated waste test results and combined with scaling laws and corrections to our understanding of the process. The resulting correlations will allow improved estimates of the WTP performance, given actual waste characterization and parametric behavior testing results.

The improved full-scale, facility-performance predictions are based on process data from single-scale (4.5-scale) and laboratory testing of the Phase 1 simulated and actual wastes performed under idealized conditions (uniform temperature, constant and uniform mixing, etc.) rather than on a series of simulation tests conducted at different scales. This approach requires the "scale-up" of the PEP process data to fullscale to be well-understood and quantifiable. Therefore, PNNL was directed to formulate and document the best analytical approaches for predicting WTP performance from PEP data.

\subsection{Purpose of This Report}

The purpose of this report is to provide the RPP-WTP project with a documented approach to analyzing PEP performance data necessary to predict the corresponding performance in the WTP. The report also provides a means to document the important decisions related to PEP scaling (e.g., the scale factor of 4.5). This report is intended to address only Phase I testing in the PEP.

\footnotetext{
${ }^{1}$ Barnes SM (Chairperson). October 23-24 (meeting date). Meeting Minutes: WTP Project Ultrafiltration Leaching Process Test Platform Scaling Recommendation Technical Review, CCN: 147164 Bechtel National, Inc., Richland, Washington.

${ }^{2}$ Markillie JR. 2007. Pretreatment Engineering Platform (PEP) Testing (Phase 1), 24590-PTF-TSP-RT-07-001, Rev. C, Bechtel National, Inc., Richland, Washington.
} 


\subsection{Objectives}

The primary objectives of this report are to:

- Define the technical basis for the approach in relating PEP performance data to the expected performance of the UFP system in the PTF (discussed in Sections 2 and 5).

- Review the rationale for designing the PEP to be $1 / 4.5$ the linear size of the PTF (discussed in Section 4).

- Document the technical basis for recommending an air sparger design (discussed in Appendix B).

- Analyze the PEP system to identify scaling issues and consider their potential impacts and mitigation approaches (discussed in Section 3.7).

- Provide technical guidance for test conduct and data requirements (discussed in Section 6).

\subsection{Notes on Using This Report}

Because the physical and chemical processes controlling the important performance attributes of the PTF, and hence of the PEP, are complex and interrelated, the application of principles of scaling in the design of equipment or interpretation of test data is correspondingly complicated. The report is organized to enable the reader to delve into the complexities at different levels of detail. The scheme is as follows.

- Two principles for relating PEP data to PTF performance are introduced in Section 2: similitude per se (2.1) and confirming the technical basis for design (2.2).

- The concepts of characteristic scales of length and time are introduced in Section 3 (3.1 and 3.2), followed by descriptions of the major physical and chemical phenomena affecting essentially all processes, viz., mass and heat balances (3.3 and 3.4). Then the major phenomena are described in more detail in terms related to major performance attributes: washing (3.5), leaching (3.6), and filtration (3.7).

- After setting the context in Sections 2 and 3, the nature of "scaling" and issues in applying its principles to the complex phenomena of the PTF and PEP are presented in Section 4, with emphasis on inevitable limitations (4.1), difficulties associated with inherently transient systems (4.2), nonideal mixing (4.3), and mixing by spargers (4.4).

- The specific rationale for the size of the PEP relative to the PTF is derived in Section 5, including both general considerations (5.1) and specific arguments for similitude in filtration performance that established the exact ratio of length scales (5.2).

- The use of PEP data is anticipated in Section 6, where we describe the kind of information to be inferred from the data and point to corresponding sections of the report providing the technical basis, but also noting that the actual analysis of the data will be determined also by the data itself-i.e., any surprises will be cause to revisit basic principles described in this report to reconsider how best to interpret and use the data.

- Technical guidance for test planning is provided in Section 7, organized around the major process steps in the PTF and their performance measures, referencing corresponding developments of ideas or relationships in Sections 3, 4, 5, and Appendix A. 
- Appendix A and Appendix B provide greater levels of detail supporting the technical sections in the main text. The technical sections in the main text are supported by sections in Appendix A, which includes essentially tutorials spanning several levels of technical depth (e.g., A.1.1 to A.1.5, A.4, and A.5) and the development of the main concepts used in the main text report (e.g., A.1.6, A.2, A.3). Appendix B is more specialized, and provides several layers of technical depth on the single topic of sparger design. 


\subsection{Technical Bases for Predicting the Performance of the PTF}

There are two principal means of predicting the performance of the PTF from operation of the PEP. First, we can apply of principles of similitude to interpret PEP data as virtual PTF data, as though we were operating the PTF. This follows the objective stated in the PEP test plan" to "perform in a similar manner to the WTP, demonstrating those aspects of the processes that meet expectations and helping to identify potential problems in the WTP." Unfortunately, there is no set of adjustable parameters that establishes full similitude between the PEP and the PTF, and therefore, not all predictions of PTF performance can be based on similitude between the PEP and PTF. Instead, we must optimize what can be deduced from similitude and dimensional analysis and apply it in terms of the simplest relationships between PTF performance and PEP data.

Second, we can predict the performance of both the PTF and PEP from their design basis-i.e., from understanding the process chemistry and process operations. The system is designed to attain specific performance objectives. Each piece of the design process involves implicitly predicting a corresponding piece of the performance. For example, a pipe is designed not to exceed a certain pressure drop by using correlations that predict the pressure drop from a given design. The design step is essentially a "backwards prediction" step in which the design is chosen so that the available basis-first principles, correlations, data - predict that the specified performance will be attained. The combination of all such correlations, first principles (e.g., mass or heat balances), and data specific to the project (e.g., leach or wash data), constitutes the basis on which the PTF is designed; its "design basis." Similarly, the combined predictions of the performance of the corresponding pieces of the PTF constitute a prediction of the performance of the PTF overall, such as the filtration rate, leach factors, and wash factors. This is the "design basis prediction."

The performance of the PEP will be compared to the design basis prediction of its performance, utilizing laboratory data such as those describing the leach rate of the simulated waste. If the actual performance is consistent with the design basis prediction, the PEP design basis is confirmed. To the extent that the PEP is designed so its performance is controlled by the same phenomena that will control the performance of the PTF, the design basis for the PEP and PTF are the same. Confirming the PEP design basis also confirms the design basis for the PTF, which confirms the validity of a design basis prediction of PTF performance.

Complementally, differences between the design basis prediction and the actual performance of the PEP provide information from which we can improve or correct the design basis, which then also improves or corrects the design basis for the PTF and thereby also improves or corrects the design basis prediction of PTF performance. This is a crucial link between the PEP and PTF by which PEP data are used to predict the performance of the PTF. These two concepts - prediction through similitude and a design basis prediction - are discussed further below.

${ }^{1}$ Markillie JR. 2007. Pretreatment Engineering Platform (PEP) Testing (Phase 1), 24590-PTF-TSP-RT-07-001, Rev C, Bechtel National, Inc., Richland, Washington. 


\subsection{Predicting the Performance of the PTF From Similitude}

The objective of the PEP, to "perform in a similar manner to the WTP, demonstrating those aspects of the processes that meet expectations and helping to identify potential problems in the WTP," requires that behavior in the PEP represents behavior in the PTF. "Similar" operation implies that phenomena and processes observed in the PEP represent those in the PTF, although they occur in a physically smaller system and where some relation is known between the rate of processes observed in the PEP and the PTF.

This objective calls for establishing and understanding "similitude" between the systems. "Similitude" and "dimensional analysis" are commonly invoked in experimental engineering and research. Some pertinent definitions are

"Similitude is the theory and art of predicting prototype performance from model observations [which] involves the application of dimensionless numbers, such as the Reynolds number or Froude number. ... The art of similitude enters the problem when the engineer must make decisions about model design, model construction, performance of tests, or analysis of results that are not included in the basic theory. [when underlying equations are known] by considering a dimensionless form of that equation, we [can] obtain a set of dimensionless parameters with which to correlate the data. [if one does not know the equations, one] must seek the dimensionless parameters by using ... dimensional analysis." 1

and

"The principal use of dimensional analysis is to deduce from a study of the dimensions of the variables in any physical system certain limitations on the form of any possible relationship between those variables. The method is of great generality and mathematical simplicity" 2

One must apply principles of similitude to determine the design and operating conditions for the PEP to represent the PTF. Thus, one objective of the design and operation of the PEP is to establish similitude with the PTF to the extent practical, notwithstanding the considerable practical constraints on establishing similitude, which are discussed in Appendix A, Section A.2.4. Essentially, the PEP is designed and will be operated to provide virtual experience in operating the UFP of the PTF because its performance and behavior are plausibly limited by the same phenomena and processes limiting the performance in the PTF.

The phenomena controlling the performance of the UFP in the PTF are described in Section 3 and in the Appendices. They are physically and chemically complex, involving chemically reacting slurries of chemically diverse particles spanning particle size distributions of several orders of magnitude.

Hydrodynamic interactions among particles can produce non-Newtonian rheological behavior. The fluid motion of greatest importance is turbulent, prominently transient, and prominently inhomogeneous.

1 Crowe CT, DF Elger, and JA Roberson. 2005. Engineering Fluid Mechanics (Eighth Edition), John Wiley \& Sons, Inc., Hoboken, New Jersey.

2 Bridgman PW. 1969. "Dimensional Analysis," in Encyclopedia Britannica (William Haley, Editor-in-Chief), Vol. 7, pp. 439-449, Encyclopedia Britannica, Chicago. (Quoted in Sonin, AA, 2001, The Physical Basis of Dimensional Analysis, Second Edition, Department of Mechanical Engineering, MIT, Cambridge, Massachusetts.) 
Therefore, while it is important to understand and optimally apply principles of similitude, it is equally important to understand and accept that no set of adjustable parameters establishes full similitude between the UFP in the PEP and the PTF and that not all predictions of PTF performance can be based on similitude between the PEP and PTF. Instead, what can be deduced from similitude and dimensional analysis must be optimized and (mainly) applied in terms of the simplest relationships between PTF performance and PEP data.

Furthermore, as discussed in Appendix A, Section A.1.3.2, the only practical parameter available to adjust to establish any aspect of similitude is the characteristic velocity, or equivalently the hydrodynamic characteristic time. Aside from being limited to affecting similitude only by adjusting the velocity, the general approach to similitude must be empirical because the system is far too complicated to extract credible conditions for similitude only from first principles (i.e., too complicated to rely on the dimensional analysis of the conservation laws described in Appendix A, Section A.1.4). Instead, we must base key decisions on dimensional analysis applied to experiments relevant to the behavior in the PTF we want to simulate in the PEP. An example is referring to correlations of settling experiments described in Appendix A, Section A.3.2.2.

On the other hand, approximate mechanistic analysis can be combined with dimensional analysis to provide useful conditions of similitude. An example is the analysis of the simultaneous effect of turbulent dispersion and settling developed in Appendix A, Section A.1.4.4.

\subsection{Predicting the Performance of the PTF From Design}

Chemical processes are designed to attain specific performance objectives. Predicting performance is inherent to the design process but this is not always obvious because it is done piecemeal, each piece limited to attaining sub-objectives of the overall performance. For example, one part of the design is developed to attain a certain heat transfer rate to meet some thermal objective, and another part is developed to attain flow rates to meet a mixing objective or a time objective, and so on. In each case, there is some technical basis for the design that could include basic principles such as mass and heat balances or principles of fluid mechanics; engineering correlations such as pressure drop; heat transfer correlations, etc. and laboratory or pilot scale data obtained by the project such as leach rates or filter flux, etc. All of these combined is the technical basis for the design of the full-scale system from which system performance is predicted to attain its performance objectives. This prediction is available at the same confidence level as for the technical basis itself.

The PTF is designed and will be operated to achieve a certain performance. Its design is the result of many analyses and calculations based on

- identifying the physical and chemical processes providing or limiting the performance;

- understanding those processes quantitatively based on first principles, standardized information, and experience and common practice; and

- configuring the design so the resulting design basis calculations predict the desired performance.

The PEP is designed using the same principles and information as is the PTF. Therefore, operating the PEP will provide data quantifying its performance that will be compared to the performance predicted from these design principles and information, including laboratory data such as those describing the leach 
rate of the simulant. If the actual performance is consistent with that predicted, the technical basis for designing both systems is vindicated. If not, the difference is the basis for reevaluating and correcting the technical basis.

To the extent that the PEP involves the same phenomena that will control the performance of the PTF, the technical basis for the design of the PEP and PTF is the same. Vindicating the correctness of the technical basis for the PEP by comparing design-based predictions to PEP data then also vindicates the technical basis for the PTF. Similarly, correcting the technical basis for the PEP design also corrects the technical basis for the PTF design. Although the physical design of the PTF is not changed, the ability to predict its performance from the technical basis - mass balances, heat balances, engineering correlations, and project laboratory and pilot data-is confirmed or improved and corrected.

The nominal technical basis of the design of the UFP of the PTF is that

- The filtration rate depends on the solids fraction in the feed, the viscosity of the liquid phase in the feed, the pressure between feed and filtrate, and the cross-flow velocity for a given filter and filter feed; however, the relationships are complicated and involve fines accumulating in the filter and potentially a filter cake accumulating on it. All of this is determined from bench scale tests and mass balances based on nominal design conditions in the PTF.

- Wash factors are the same as those measured in laboratory tests if, in the laboratory, one imposes the same solids fraction and liquid composition as in the PTF prior to equilibration of the solid and liquid phases.

- Leach factors are the same as those observed in laboratory tests if, in the laboratory, one imposes the same reactive species, with the same particle size distribution, at the same temperature, with the same initial reagent concentrations as in the PTF.

- Mass balances and heat balances are simple control volume implementations of the conservation of mass and energy assuming uniform concentrations and temperatures in the tanks.

Section 3 describes the physical and chemical processes involved and the reasons the actual technical design bases might differ from ideal behavior implicitly assumed in the above nominal set.

The fundamental issue to be resolved by the PEP has been formally stated in the WTP project as "The chemistry has been found to work in the lab... but not in a large scale. Although large-scale mixing tests showed good mixing in the vessel, the heating and chemical reaction rates were not tested, and the design's effectiveness was not definitively demonstrated." This concise statement contains five component issues that are addressed as follows.

1. Process Chemistry - the most fundamental part of the design basis is the chemistry itself, which is revealed through laboratory testing of actual waste, while actual waste cannot be processed in the PEP. The process chemistry is determined in other tasks and is not part of the scope of the PEP, per se.

\footnotetext{
${ }^{1}$ Barnes S and R Voke. 2006. Issue Response Plan for Implementation of External Flowsheet Review Team (EFRT) Recommendations - M12, Undemonstrated Leaching Process, 24590-WTP-PL-END-06-0024, Rev 0., Bechtel National, Inc., Richland, Washington.
} 
2. Scale - at laboratory scale, uniform temperatures and concentrations are attained on time scales that are short compared to the elapsed time of chemical processes, but this is not ensured at full-scale. The PEP is designed at a scale sufficiently large to reveal non-uniform distributions of mass and energy that might occur as the size of the system increases from laboratory to full-scale.

3. Mixing - in laboratory experiments, mixing is easy to accomplish and usually taken for granted, but in a full-scale process often good mixing is difficult to attain, difficult to characterize, and cannot be taken for granted. The PEP is designed to employ the same physical mixing processes as in the PTF.

4. Design - because the process chemistry is the essence of the UFP, one must be careful to recognize it is not the entire basis; in particular, the performance of the PTF is predicted based on both the process chemistry and on mass balances over control volumes in which uniform concentrations and temperatures implicitly are presumed. This is essentially issue 3 above.

5. Demonstration - the principal purpose of the PEP is to resolve the "undemonstrated leaching processes" issue through demonstration. The PEP is designed to provide the same basic process functions as the UFP system in the PTF. Providing this while addressing issues 2, 3, and 4 provides a meaningful demonstration in which successful operation and satisfactory performance basis implies successful operation and satisfactory performance of the PTF.

One objective of this report is to analyze the PEP system to identify scaling issues and consider their potential impacts and mitigation approaches. The context for identifying "scaling issues" for the PEP is ensuring that operation of the PEP and analysis of resulting data address issues 2 through 5 above. 



\subsection{Phenomena Controlling the Performance of the PTF}

Application of both similitude and the design basis to predict the performance of the PTF is based on the chemical and physical phenomena controlling the performance. The essential process functions involving these phenomena are described below.

\subsection{Process Functions}

The essential performance measures of the PTF are the fractions of components in the tank waste that are delivered to HLW and low-level waste (LAW) vitrification. These are determined by a combination of physical and chemical separation steps; the performance of each can be similarly characterized in terms of the fractions of components delivered to the next step in the PTF. A crucial part of the PTF is the UFP, in which dissolved components are delivered to LAW vitrification (after subsequent chemical separation of Cs to HLW) and undissolved aluminum and chromium are converted to soluble forms and also delivered to LAW vitrification. While solid and dissolved components are separated, ultimately these separations are physical and accomplished by filtration.

Figure 3.1 depicts the essential functions of the UFP flowsheet, which are quantified by three performance measures: wash factors, leach factors, and the filtration rate. The functions are described for a simplified set of components: water (alkaline), dissolved species, soluble solids, aluminum solids, $\mathrm{Al}(\mathrm{OH})_{4}{ }^{-}$from leaching the aluminum solids, chromium solids, chromate from leaching the chromium solids, and residual solids (residual solids not leached). Components of the various unit operations are identified in the bulleted boxes. "Residual solids" are insoluble and are not converted to a soluble form

during the process. Figure 3.2 depicts a simplified control volume for mass balances. Figure 3.3 presents a more detailed view of the process operations and the evolution of waste components. Figure 3.3, as presented, corresponds to caustic leaching following a concentration step. Because this figure does not allude to equipment, the alternative case of "upfront leaching" corresponds to the same steps except excluding the initial concentration step.

\subsection{Considerations of Scale Common to All Process Steps}

Several effects of scale - referring generally to all "scales" associated with phenomena affecting the performance - apply to many of the process steps in the UFP. These are discussed below. Considerations of scale - i.e., "time scale" (often, velocity choices) — specific to individual process steps are discussed in Section 7.

\subsubsection{Length Scale}

Given geometric similitude, the length scale determines the size of all volumes and areas affecting the process: areas are proportional to the length scale squared, and volumes are proportional to the length scale cubed. Complete geometric similitude implies that the relative sizes of all objects affecting system performance are the same between systems. "Complete" implies the sizes of particles and bubbles relative to the size of the system are the same between systems. However, these sizes are controlled by the physical and chemical attributes of the materials processed in the systems, which, in combination with 


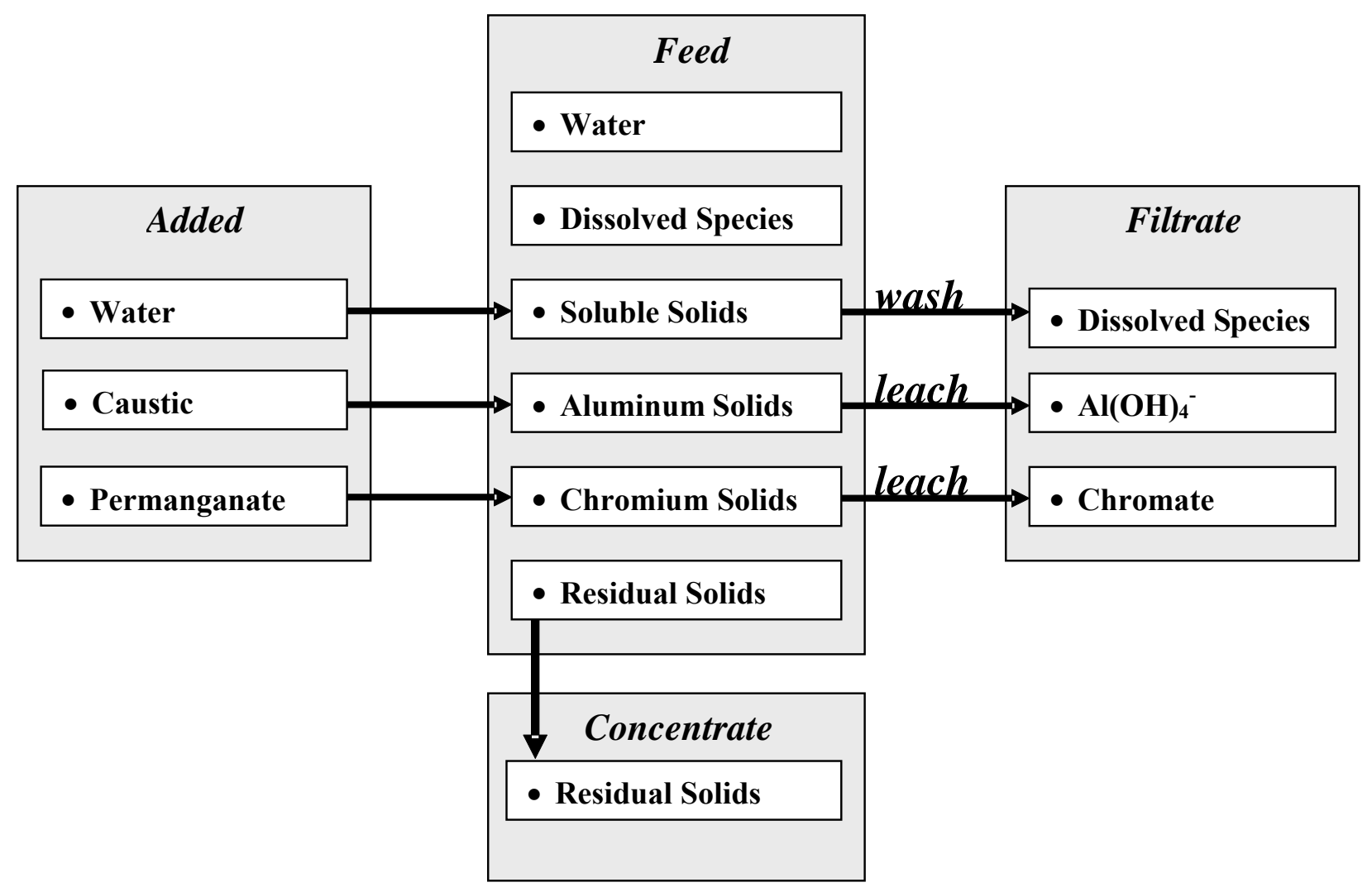

Figure 3.1. Simplified Depiction of UFP Functions

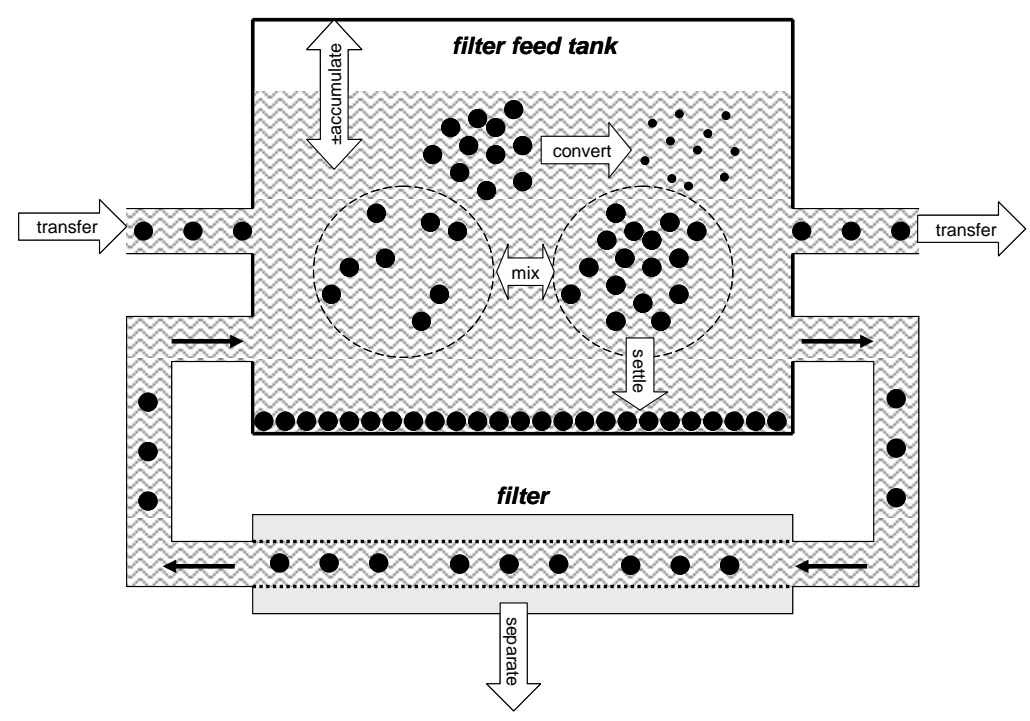

Figure 3.2. Mass Balance for a Control Volume (Tank) 
To Tank Transfer Feed

- Water

- Dissolved Species

- Aluminum Oxide

- Chromium Oxide

- Intractable Solids
In Tank

Concentrate Solids

- Water

-.....Dissolved Species

- Soluble Solids

- Aluminum Oxide

- Chromium Oxide

- Intractable Solids
From Tank Filter

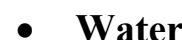

- Dissolved Species

Add Reagent

- Caustic

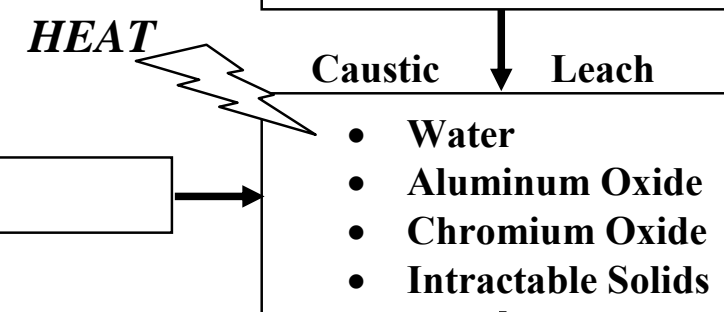

Reconcentrate Solids

Filter

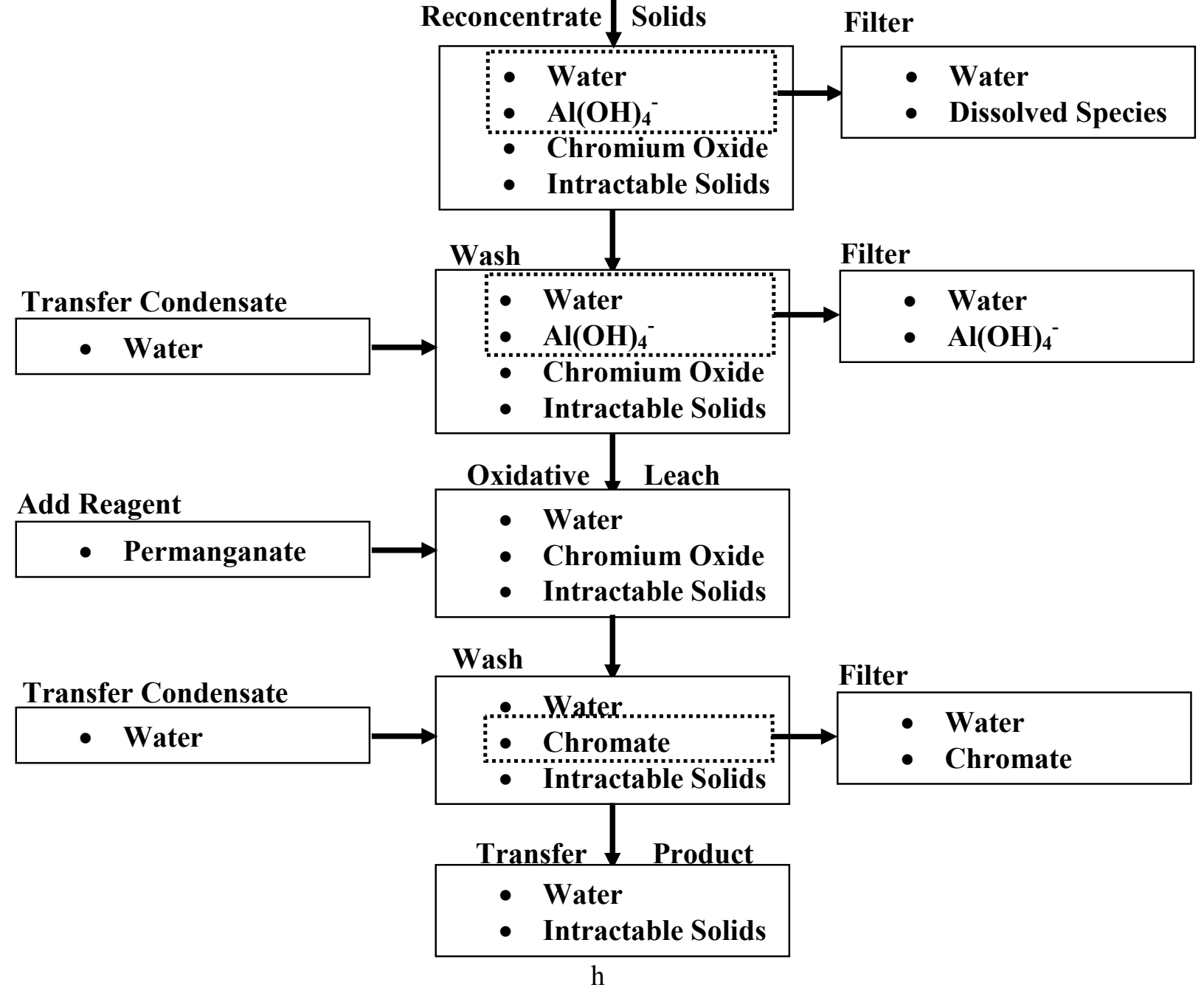

Figure 3.3. UFP Steps 
enforcing thermal and chemical similitude, are difficult if not impossible to adjust to enforce the desired size ratios. Thus, for practical reasons, the "length scale" pertains only to the size of features of process equipment.

Therefore, because the size and shape of the equipment in the PEP cannot be changed between tests, "length scale" is not a test choice if one maintains geometric similitude. However, the size of individual components can be changed where, for specific reasons, we accept the lack of geometric similitude in interpreting the results.

\subsubsection{Time Scale}

We can change the ratio of time between systems required for corresponding processes to occur; therefore, "time scale" denotes the time required to accomplish a specified change. Such changes can be

- hydrodynamic, e.g., a characteristic volume divided by a characteristic volumetric flow rate (volume/time);

- process control, e.g., based on the time allotted between beginning and ending a step such as heating or a pulse-jet mixer (PJM) pulse;

- physicochemical, e.g., based on the motion of particles or bubbles;

- chemical, e.g., accomplishing a given conversion by a chemical reaction.

We must choose a characteristic change as a basis for defining the time scale. The time scale can then be adjusted by adjusting a flow rate or elapsed time between events.

The volumetric flow rate in the filter loop is the sum of the volumetric flow rates in the individual filter tubes. The velocity in the filter tubes is the cross-flow velocity, which is matched between the PEP and PTF. The volumetric flow rate in the filter loop is determined by the number of filter tubes. The PEP is designed to allow adjusting the number of filter tube bundles connected in series, but does not allow adjusting the number of filter tubes in a bundle. Therefore, the volumetric flow rate in the filter loop is fixed during filter testing. The velocity at the filter loop inlet to the vessel is the ratio of the volumetric flow rate to the nozzle area (the cross-sectional area of the filter loop pipe as it enters the tank), and the latter is fixed by the length scale and choosing to maintain geometric similitude. Therefore, the inlet velocity is fixed, and the velocity at the filter loop inlet nozzle in the vessel is not available as a test choice during filter testing. The PJM nozzle velocity is determined by the volume of liquid expelled divided by the drive time; this is available as a test choice by adjusting the PJM drive time.

Taking a specific adjustable velocity as our characteristic velocity $\mathrm{u}$, for example the PJM nozzle velocity, and any length for which we maintain geometric similarity between the PTF and PEP as our characteristic length $\mathrm{L}$, then we can express the velocity-based kinematic in the form of the product (L)(un), where $\mathrm{u}$ is velocity and $\mathrm{n}$ is an exponent associated with the time scale. This links the time scale to the length scale through the exponent $\mathrm{n}$. For example, if we established a time scale based on the yield Reynolds number, then $\mathrm{n}=0$ and the time scale does not depend on the length scale: we would be matching $\mathrm{uL}^{0}$, for which the ratio of velocities is not a function of the ratio of lengths. Then we would match the velocities to match the time scales. If instead we established a time scale based on 
power/volume, which is proportional to $u^{3} / \mathrm{L}$, we would be matching $u L^{-1 / 3}$, in which case $n=-1 / 3$ and the time scale does depend on the length scale. In that case, to establish equal time scales we must to adjust the ratio of velocities accordingly.

As discussed in Appendix A, Section A.3.1, we expect the uniformity of suspended solids to "scale" as the ratio of the particle settling velocity to the characteristic velocity imposed on the system. Because the settling velocity is a property of the slurry and because such properties will be matched between the PEP and the PTF, we expect the ability to maintain a uniform solids concentration is matched by matching the velocities imposed in the PEP and the PTF. Put into a more general context, this corresponds to matching the product $u L^{0}$.

As discussed in Appendix A, Section A.3.2.2, in steady-state systems not dominated by nonNewtonian behavior, the concentration of a slurry with given particle attributes that can be kept suspended has been found empirically to be a function of the product of the velocity imposed and the length scale to approximately the $-1 / 6$ power. We do not have a firm basis to conclude the same functional form applies to suspension by transient jets, yet currently we have no better premise available from the engineering literature as a basis for anticipating the ability to suspend solids in the PEP or PTF. To adopt this criterion, we would match the product $u L^{-1 / 6}$.

Industrial experts advise that the power/volume ratio is often used as a criterion for good mixing and suspension of solids in industrial blenders and stirred-tank reactors. As discussed in Appendix A, Section A.1.4.4, this would require matching the product $u L^{-1 / 3}$ between the PEP and the PTF.

As is discussed in Section A.3.3, an energy analysis of the effects of density differences in organized transient flows leads to an argument for matching $\mathrm{uL}^{-1 / 2}$ between systems in order to observe a representative stratification due to limitations (if any) of the "cloud height" during a PJM cycle.

Finally, the relative intensity of turbulence - the ratio of the magnitude of turbulent fluctuations in velocity to the time-averaged velocity - is determined by the Reynolds number, albeit not necessarily strongly. The Reynolds number would be matched between systems by matching the product $u L^{l}$.

\subsubsection{Thermal and Chemical "Scale"}

We can define the thermal and chemical "scale" of a system by the range of temperatures and concentrations within it. Because interactions between physical and chemical attributes depend on both temperature and concentration, they are exceedingly complex. The only practical choice to provide thermal and chemical similitude is to match the thermal and chemical scales between systems. Therefore, these scales are not available to be varied between tests.

\subsection{Mass Balances}

UFP tanks constitute control volumes for mass balances used to predict the masses of liquid and solids resulting from the addition, removal, or conversion of components in the liquid and solid phases. The mass balances used to compute the disposition of components being washed or leached are described further in Sections 5.3 and 5.4. The wash and leach factors are computed directly from the dissolved (dissolved during washing or converted during leaching) masses of solids removed in filtrate compared to the entering masses of the same solids. 
The foundations for predicting the behavior of the PTF based on information obtained in the laboratory, in the literature, or from engineering experiments are mass and heat balances over the process tanks. The main elements of mass balances for UFP tanks are depicted in Figure 3.2 and include

- accumulation of liquid

- accumulation of solids

- transfer of liquid and solids

- settling of solids from the control volume to the bottom boundary

- mixing

- conversion of solids to dissolved forms

- separation of liquid from solid.

The mass concentrations can only be measured through samples. However, no mass transfers across solid boundaries, thereby facilitating calculating mass concentrations from mass balances.

Mass balances for specific chemical components are composites of several coupled separate balances between the liquid volume, the composition of the liquid expressed as masses of dissolved solids, and the masses of undissolved solids, as follows.

$$
\begin{gathered}
\frac{d m_{L}}{d t}=\frac{d}{d t}\left(V \overline{\phi_{L}}\right)=\phi_{L, \text { in }} F_{\text {in }}-\phi_{L, \text { out }} F_{\text {out }}-F_{\text {filtrate }} \\
\frac{d m_{i}}{d t}=\frac{d}{d t}\left(V\left(1-\overline{\phi_{S}}\right) \overline{C_{i}}\right)=\left(1-\phi_{S, \text { in }}\right) C_{i, \text { in }} F_{\text {in }}-\left(1-\phi_{S, \text { out }}\right) C_{i, \text { out }} F_{\text {out }}-C_{i, \text { filtrate }} F_{\text {filtrate }} \\
\frac{d m_{j}}{d t}=\frac{d}{d t}\left(V \overline{\phi_{S}} \overline{C_{j}}\right)=F_{\text {in }} \phi_{S, \text { in }} C_{j, \text { in }}-F_{\text {out }} \phi_{S, \text { out }} C_{j, \text { out }}
\end{gathered}
$$

Where $m_{L}, m_{i}, m_{j}$ are the masses in a tank of liquid, dissolved species $\mathrm{i}$, and solids species $\mathrm{j}$; $V$ is the volume of slurry in the tank; $\overline{\phi_{S}}, \phi_{S, \text { in }}, \phi_{S, \text { out }}$ are the volume fractions of solids averaged over the volume of a tank, the volume fraction in a stream entering the tank, and in a stream exiting the tank; $F_{\text {in }}, F_{\text {out }}, F_{\text {filtrate }}$ are the volumetric flow rates (volume/time) of streams entering and exiting a tank and of filtrate; and $\overline{C_{i}}, C_{i, i n}, C_{i, i n}, C_{i, f i l t r a t e}$ are the volume fractions of solids averaged over the volume of a tank, the volume fraction in a stream entering the tank, in a stream exiting the tank.

For multiple dissolved species $i$ or solids species $\mathrm{j}$ the equations repeat accordingly. In any event, additional relationships are needed to relate these mass balances, namely

$$
\phi_{S, \text { out }}=f\left(\overline{\phi_{S}}, \phi_{S, i n}, \text { flow field }\right) \text { and } C_{j, \text { out }}=f\left(\overline{C_{j}}, C_{j, i n}, \text { flow field, chemical reactions }\right)
$$

where $f$ is some function relating the outlet solids fraction to the average in the tank and the fraction in the inlet(s) and relating the solids fraction to the flow field in the tank and chemical reactions that transfer species from the solid to dissolved state. Most engineering design basis mass balances are used assuming these functions are simply

$$
\phi_{S, \text { out }}=\overline{\phi_{S}} \text { and } C_{j, \text { out }}=\overline{C_{j}}
$$


This is a practical definition of ideal mixing; the solids volume fraction and the composition of the solids (averaged over all particles) are uniform over all points including exits from the tank. But depending on the flow field, mixing may be imperfect due to failure to suspend solids, failure to maintain a uniform solid volume fraction over suspended solids, or failure to sustain volumetric uniformity of chemical reactions in a tank.

That is, mass balances over control volumes are straightforward whenever the distribution of mass is uniform. In that case, the effluent mass flow is simply the product of the effluent volumetric flow rate and the mass concentration is assumed to characterize all of the volume. However, if the mass distribution is not uniform, there must be some means for calculating the mass departure of the mass concentration in the effluent from the average for the tank, which will depend on quantifying phenomena such as mixing and settling.

Nominally, wash and leach factors for either the PTF or PEP are computed directly from the dissolved (dissolved during washing or converted during leaching) masses of solids removed in filtrate compared to the entering masses of the same solids. In the case of the PTF, these are design calculations. In the case of the PEP, they are part of analyzing the data.

The mass balances used to compute the disposition of components being washed or leached are described further in Appendix Sections A.2 and A.3.

\subsection{Heat Transfer and Condensate}

The main use of heat balances for the PTF will be to relate the expected times to heat or cool the contents to the mass of the slurry and the flows and temperatures of process streams, for the purpose of process control. The temperature is relatively simple to measure at specific locations in a tank. It is far less straightforward to determine at what point to measure the temperature so it represents the volume average for the tank, or equivalently to determine the representativeness of a value measured at any specified location. The temperature would be inherently uniform if any heat added or lost occurred uniformly over the tank, but actually heat will be added at a single point during caustic leaching and removed non-uniformly over the walls of the tank and due to evaporation and especially sparging.

The main elements of heat balances for UFP tanks include

- accumulation of heat (change in temperature)

- transfer of hot/cool slurry

- mixing

- condensation of steam

- evaporation of water

- conduction of heat through boundaries, including heat exchangers.

The accumulation and transfer contributions to a heat balance calculation also are simple if the temperature is uniform, but contributions from conduction and evaporation, especially if incidental rather than the result of an intentional process, are difficult to estimate. 


\subsubsection{Effect of Size on Dilution by Condensation}

Heat transfer by conduction through boundaries is predicted using heat transfer coefficients describing conduction in solid boundaries, forced convective heat transfer in fluids at a boundary, and natural convection heat transfer in fluids at a boundary. The flow of heat can be in parallel through alternative paths, in series through multiple paths, or both; each heat flow step along a flow path is characterized by a heat transfer coefficient. An overall heat transfer coefficient describing the ratio of total heat flow to total temperature difference is constructed from individual coefficients given the geometric and material properties describing the heat transfer path(s). The dependence on characteristic length and velocity of the coefficients and of the predicted heat flow is addressed using dimensional analysis as discussed in Appendix A, Section A.5.2. Approximately, the heat balance for a vessel with simultaneous steam injection and sparging

$$
m C p \frac{d\left(T-T_{0}\right)}{d t}=\Delta H^{v a p}\left(\frac{d m_{\text {inject }}}{d t}\right)-\Delta H^{v a p}\left(\frac{d m_{s p \text { arge }}}{d t}\right) f(T)-A_{0} h_{0}\left(T-T_{0}\right)
$$

where $m$ is the thermal mass, $C_{p}$ is the specific heat, $T$ is the temperature, $T_{0}$ is the ambient temperature, $\Delta H^{v a p}$ is the heat of vaporization, $\mathrm{dm}_{\text {inject }} / \mathrm{dt}$ is the mass rate of injection of live steam, $\left(d m_{\text {sparge }} / d t\right)_{\text {sparge }}$ is the mass rate of injection of sparge gas, $f(T)$ is a complicated function involving the growth of sparge bubbles, $A_{0}$ is the area of heat transfer across boundaries, and $h_{0}$ is the overall heat transfer coefficient at the boundary.

Consider the case of maintaining a steady temperature. Setting the derivative to zero and rearranging,

$$
\Delta T=\lambda(1-F(T))
$$

where

$$
\Delta T=T-T_{0} \quad \lambda=\frac{\Delta H^{\text {vap }}}{A_{0} h_{0}}\left(\frac{d m_{\text {inject }}}{d t}\right) \quad F(T)=\left(\frac{d m_{\text {sp arge }}}{d m_{\text {inject }}}\right) f(T)
$$

Differentiating with respect to $\lambda$,

$$
\frac{d \Delta T}{d \lambda}=1-F(T)-\lambda \frac{d F(T)}{d T} \frac{d \Delta T}{d \lambda} \quad \therefore \frac{d \Delta T}{d \lambda}=\frac{1-F(T)}{1+\lambda(d F / d T)}
$$

The difference $1-F(T)$ is positive because the heat loss due to sparging must be less than the heat added by injecting steam. The derivative $d F / d T$ is $d f / d T$ is positive due to the effect of the vapor pressure and of temperature on the vapor pressure. Therefore, the steady-state temperature difference increases with $\lambda$, and hence decreases with the area and with the size if all else is held constant (injection rate, sparge rate) because, as noted above, the heat transfer coefficient is only a weak function of the size of the system. Therefore, the effect of heat loss through boundaries seen at a smaller scale will be a conservative estimate of the effect at a larger scale. 
The steady-state heat balance can also be written in terms of the mass of water evaporated by sparging, as follows.

$$
\Delta H^{\text {vap }}\left(\frac{d m_{\text {added, injecting }}}{d t}\right)-\Delta H^{\text {vap }}\left(\frac{d m_{\text {lost, sparging }}}{d t}\right)=A_{0} h_{0} \Delta T
$$

Rearranging, the rate of accumulation of condensate is

$$
\frac{d m_{\text {water }}}{d t}=\frac{d m_{\text {added, injecting }}}{d t}-\frac{d m_{\text {lost, sparging }}}{d t}=\frac{A_{0} h_{0}}{\Delta H^{\text {vap }}} \Delta T
$$

Hence, for a given $\Delta T$ being maintained, the rate of accumulation of water is proportional to the area, i.e., to the square of the size of the system. On the other hand, the mass of slurry being diluted by the accumulating water is proportional to the cube of the system. Therefore, the rate of dilution expressed as the fractional mass added per time, i.e., the dilution per time, increases in inverse proportion to the size of the system if the overall heat transfer coefficient $h_{0}$ is the same between systems. Consequently, during caustic leaching, the concentrations of both reagents and reaction products will be less in a smaller system than in a larger system. The net effect on the chemical conversion needs to be determined from the reaction rate law determined in the laboratory.

In addition, the rate of increase in the volume of the slurry during caustic leaching will be greater in proportion to the tank volume in a smaller system than in a larger system, which must be addressed in operational plans for the smaller system.

\subsection{Washing}

\subsubsection{Chemical Description}

Washing separates dissolved components from solids. There are two steps: first, solids components are concentrated by separating them from dissolved components in the waste while waste is added; second, water is added (as condensate collected from process operations) to the remaining solids to replace interstitial liquid, which also dissolves any soluble solids in the slurry.

The first step is adding waste slurry and removing liquid from the tank holding the slurry, whether or not all of the solids are suspended as a slurry or reside on the tank floor. The separation is essentially perfect; only the filtration rate is affected by the scale of the system.

The second step consists of replacing with condensate the interstitial fluid among solid particles and potentially dissolving soluble solids among the solid particles. Although condensate is not added continuously, we treat this approximately as though condensate is added and filtrate is removed continuously at volumetrically equal rates such that the volume of the liquid phase remains constant. The replacement step is affected potentially by the uniformity in the distribution of the added condensate over the interstitial liquid volume of the slurry and the uniformity in the distribution of the solids mass over the volume of the slurry. The first affects the local fraction of interstitial liquid replaced by condensate; the second affects the fraction of any soluble solids locally dissolved by the added condensate. 


\subsubsection{Design Basis Wash Factors}

The relationship of wash factors to operational parameters is developed in Appendix A, Section A.2.1. For the design basis, which is ideal mixing, the wash factor is

$$
\omega=1-\frac{1}{\psi} \frac{\chi_{0} e^{-\left(\lambda-\lambda_{\min }\right)}}{\left(1-\phi_{S 0}-\phi_{I 0}\right) \chi_{0}+\phi_{S 0}}
$$

where $\chi_{0}$ is $\mathrm{C}_{0} / \rho_{\mathrm{S}}, \mathrm{C}_{0}$ is the initial concentration of dissolved species in the liquid phase of the waste and $\rho_{\mathrm{S}}$ is the density of the soluble solid, $\lambda$ is the condensate ratio $\Delta V / V, \lambda_{\min }$ is the minimum value of $\lambda$ for which all soluble solids are dissolved, $\varphi_{\mathrm{S} 0}$ is the volume fraction of soluble solids in the tank waste, $\varphi_{\mathrm{I} 0}$ is the volume fraction of insoluble solids in the tank waste, and $\psi$ is the concentration ratio $V_{0} / V$, where $V$ is the volume of slurry in the UFP vessel, $V_{0}$ is the volume of waste concentrated in the UFP, and $\Delta V$ is the volume of concentrate added to wash the concentrated solids.

\subsubsection{Effects of Settled or Stagnant Regions}

Several possible cases of non-ideal mixing are considered in Appendix A, Section A.2.1.2. Based on the hypothesized departures from ideal mixing, the wash factor is reduced to

$$
\omega_{\text {unmixed }, \text { settled }}=1-\frac{1}{\psi} \frac{\beta \phi_{S, \text { settled }}+\alpha \chi_{0}+(1-\alpha) \chi_{0} e^{-\frac{\lambda-\lambda_{\min }}{1-\alpha}}}{\left(1-\phi_{S 0}-\phi_{I 0}\right) \chi_{0}+\phi_{S 0}}
$$

where $\alpha$ is the fraction of the slurry volume that is unmixed, and $\beta$ is the fraction of the slurry volume that is settled particles.

\subsection{Leaching}

\subsubsection{Chemical Description}

Leaching is a chemical conversion of insoluble solids to a soluble form followed by separation of the dissolved soluble form from residual solids. The separation is essentially the same as for washing: concentration of the residual solids by removing saturated liquid, and potentially addition of condensate to promote further dissolution of the converted solid form if not already completely dissolved. The leach factors are the mass fraction of the initial mass of the target insoluble solid that is removed from the waste by filtration. This is determined by the fraction of the solid converted to the soluble form, the fraction of the soluble form dissolved, and the fraction of the dissolved soluble form removed as filtrate. Technically, leach factors depend on both the chemical conversion and the wash factor for the resulting dissolved form. However, because the separation of the dissolved form is inherent to the leaching function, we describe the combined steps using a single factor. 


\subsubsection{Ideal Mass Balance}

For uniform temperature and concentrations in the leaching vessel, the dependence of leaching time on the chemical conversion attained is shown in Appendix A, Section A.2.2.1 to be

$$
\frac{\rho_{S} A_{S}(0) r(0)}{m_{s, 0}} t=F_{X}(X)=\int_{0}^{X} \frac{d X}{\widetilde{A}(X) \widetilde{r}\left(X, \phi_{S 0}\right)}
$$

where $X$ is the fractional conversion, $t$ is time, $T(t)$ is temperature as a function of time, $C_{R 0}$ and $C_{P 0}$ are the initial concentrations in the liquid of the reagent $(\mathrm{OH})$ and reaction product $\left(\mathrm{Al}(\mathrm{OH})_{4}^{-}\right)$, and $\varphi_{\mathrm{SO}}$ is the initial value of the volume fraction of reactive solids and other parameters are explained in the appendix. The function $\widetilde{A}$ is a dimensionless particle area distribution function, and $\widetilde{r}$ is a dimensionless reaction rate law. Inverting this relationship, the conversion depends on time according to

$$
X=F_{X}^{-1}\left(F_{X}(X)\right)=F_{X}^{-1}\left(\frac{\rho_{S} A_{S}(0) r(0)}{m_{s, 0}} t\right)=F_{X}^{-1}(\tau)
$$

which can be written functionally as

$$
X=X\left(t, T(t), C_{\mathrm{R} 0}, C_{P 0}, \phi_{S 0}\right)
$$

where the functional form of $X$ is as developed above, which includes the reaction rate model that must be determined from laboratory data. The concentration and volume fraction ratios are affected by the mixing in the tank.

\subsubsection{Effects of Uniformity}

Rearranging, the departure of the average conversion from the conversion that would occur if the parameters occurred uniformly at their average values is

$$
\delta X=\bar{X}-X\left(t, \bar{\xi}_{i}\right)=\frac{1}{2} \sum_{i} \Theta_{i} \int_{m(0)}\left(\delta \xi_{i}\right)^{2} \frac{d m(0)}{m(0)}
$$

The integrals over the second variations $\left(\delta \xi_{i}\right)^{2}$ are inherently positive. Hence, the effect of the variations on the average conversion depends on both the magnitude of the variations and the sign of the second derivatives $\Theta_{i}$ of the conversion $X$ relative to the parameters $\xi_{i}$.

In summary, $\Theta_{T}$ is positive and $\Theta_{\varphi}$ is negative. Therefore, for a given average temperature, a nonuniform temperature causes a conversion that is greater than for a uniform temperature. Conversely, for $a$ given average initial volume fraction of reactive solids, a non-uniform initial distribution of the volume fraction causes a conversion that is lesser than for a uniform solids volume fraction. That is, these are corrections that must be made to the conversion predicted based on the average temperature and average solids volume fractions, which are subject to measurement error. 


\subsection{Filtration}

The filtration rate is affected both by the accumulation over time of fines in the filter media and any filter cake on the filter media. Because the PEP will utilize full-scale, prototype filter tubes operated with the prototype trans-membrane pressure and prototype cross-flow velocity, the remaining issues are the filter area (number of tubes per bundle) and similitude in time related to the fine and coarse solids in the filter feed. The feed properties change with time through a mass balance over the filter feed tank and filter. The mass balance is affected by the uniformity of solids over the volume of the feed tank if the outlet concentration from the tank differs significantly from the average concentration in the tank.

\subsubsection{Conceptual Model}

The filtrate flux (mass flow rate per area) is a hydrodynamic phenomenon controlled by the physical conditions in and on top of the filter media. The media and its proximate environment is depicted in Figure 3.4.

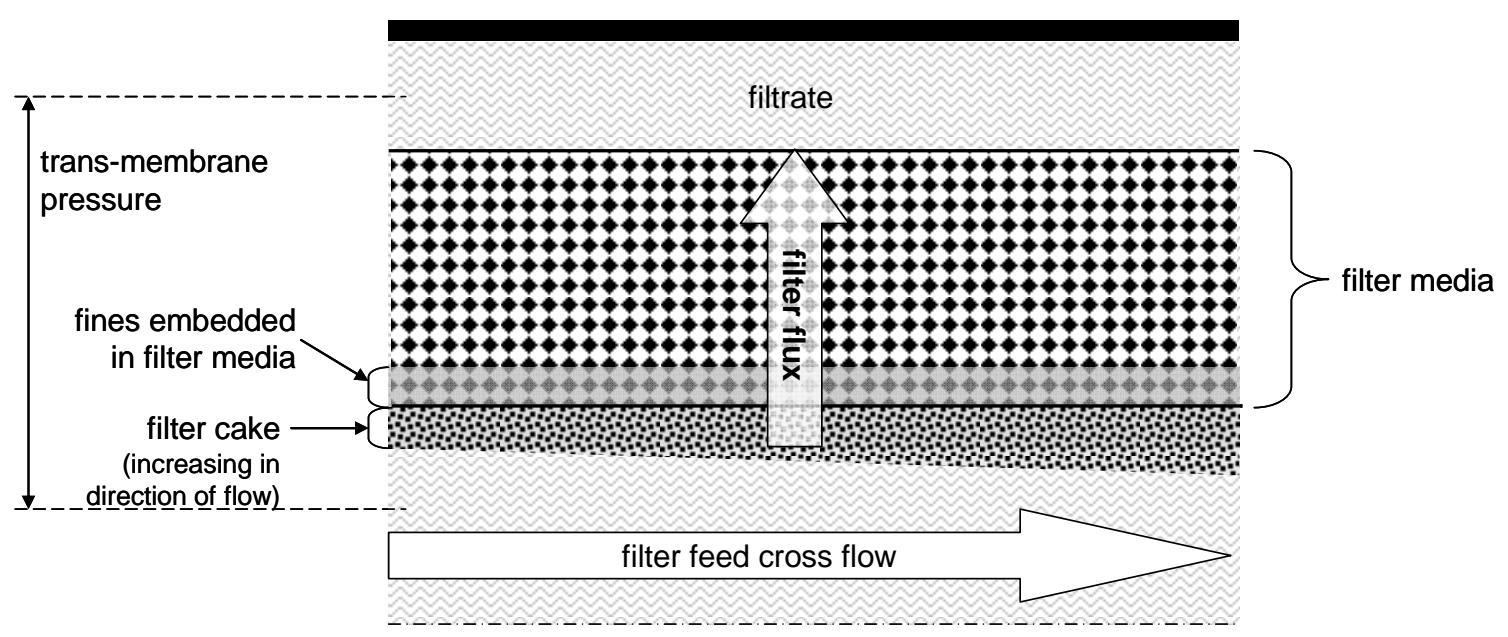

Figure 3.4. Filtration Hydrodynamic Environment

The filtrate moves under pressure from the filter feed, which flows across the surface of the media through a filter cake (when one exists) consisting of solids too coarse to enter the filter media, through a portion of the filter media partially filled by solids fine enough to follow the filtrate some distance into the filter media and through it to the filtrate collection annulus surrounding it. The cross flow is designed to create a shear stress in the filter feed at the surface of the filter to minimize the accumulation of the filter cake.

The filtrate passes through three hydrodynamic domains: 1) filter cake; 2) filter media partially occluded by fines; and 3) un-occluded filter media. Each domain is characterized by an apparent hydrodynamic permeability relating the flux to the local radial pressure gradient. As time passes, fine solids too small to be rejected by the filter media are carried by the filtrate into the media and become caught within it, thereby creating a region of permeability reduced from that of the un-occluded media. The cross flow prevents coarse solids separated from the filtrate from accumulating on the surface of the filter. However, for sufficiently large solids concentrations, the filter flux carries solids to the surface faster than the cross-flow can remove them, at which point a filter cake begins to form. 
As time passes, the loss of filtrate from the feed tank increases the solids loading in the feed, which increases the tendency for solids to accumulate on the media. Eventually the solids loading increases sufficiently that a filter cake forms. This adds a pressure drop along the path of the filtrate that decreases the filter flux for a given total pressure drop available between the inside and outside of the filter tube, denoted the trans-membrane pressure. Finally, when a filter cake exists, the ability of the cross flow to remove solids decreases in the direction of flow because any accumulated solids stripped from the surface add to the local solids loading immediately down-flow, so that in the slurry adjacent to the filter cake the effective solids loading increases in the direction of flow. The local rate of growth of the filter cake increases with the solids concentration in the adjacent slurry. Therefore, the thickness of the filter cake tends to increase in the direction of flow, such that the filter flux tends to decrease in the direction of flow once a filter cake forms. In combination, these phenomena determine the filter flux and how it varies with time for a given solids concentration in the filter feed.

Through a mass balance over the filter feed tank, the filter flux determines how the solids concentration in the feed varies with time. The mass of solids in the tank distributes roughly uniformly over the filter area. Hence, the thickness of fines in the filter or cake on the filter is proportional to the ratio of solids mass to filter area.

\subsubsection{Complexity of Constituent Phenomena}

As discussed in Appendix A, Section A.2.3 (see for the nomenclature), even the simple model developed of the progression of the filter flux involves five transient mass balances (differential equations) in five dependent functions of time, concentration of fines, concentration of coarse solids, thickness of fines accumulated in the filter media, and thickness of filter cake on the media. These equations have solutions for the concentrations of the form

$$
C_{-,+, L}=f\left(\tau ;\left(A_{L} / V_{S 0}\right) C_{F-,}\left(A_{L} / V_{S O}\right) C_{F+}, \varepsilon_{X}\right)
$$

and for the depths of the form

$$
\delta_{-,+}=f\left(\tau ;\left(A_{L} / V_{S 0}\right) C_{-,}\left(A_{L} / V_{S 0}\right) C_{F+}, \varepsilon_{X}\right)
$$

Therefore, the functional dependence of the filter flux is

$$
\tilde{u}_{F}=f\left(\left(A_{F} / V_{S 0}\right) u_{F 0} t ;\left(A_{F} / V_{S 0}\right) C_{-},\left(A_{F} / V_{S 0}\right) C_{+}, \varepsilon_{X}\right)
$$

where $A_{F} / V_{S 0}$ is the ratio of filter area to initial volume (or mass) of solids.

\subsubsection{Mass Balance Similitude}

The dimensionless transient mass balances describing the key phenomena affecting the filtration rate show similitude as established by the following:

- Between the PEP and the PTF, match the properties of the solids being filtered

- Between the PEP and the PTF, match the ratio of filter area to initial mass of solids being filtered.

The resulting filter flux, compared to that for a clean filter measured in the PEP, will decrease as the same function of dimensionless time as in the PTF. By using the same filter media and matching the 
filter area to the initial solids mass, the ratio of dimensionless times will be the ratio of actual time. The resulting filter flux, compared to that for a clean filter measured in the PEP, will evolve with time in the PEP as in the PTF.

If similitude is established as described above, the filter flux measured in the PEP is a prediction of that expected in the PTF at the same times elapsed after establishing a clean filter. 


\subsection{Scaling Issues}

One of the objectives of this report is to analyze the PEP system to identify scaling issues and consider their potential impacts and mitigation approaches. These include at least the following.

\subsection{Limits on Application of Principles of Similitude}

As is discussed in Section 2.1, the phenomena controlling the performance of the UFP in the PTF are physically and chemically complex. Therefore, while it is important to understand and optimally apply principles of similitude, it is equally important to understand and accept that no set of adjustable parameters establishes full similitude between the UFP in the PEP and the PTF and therefore accept that not all predictions of PTF performance can be based on similitude between the PEP and PTF. Consequently, we must optimize what can be deduced from similitude and dimensional analysis and (mainly) apply it in terms of the simplest relationships between PTF performance and PEP data.

\subsection{Hydrodynamics of Transient Jets}

The pulse-jet mixers, as the name implies, utilize transient submerged jets to agitate fluid in a tank. We have drawn inferences about the resulting mixing from information available about steady jets and applied principles of similitude and dimensional analysis to estimate the functional form of the dependence of mixing performance on length and time scales, but these inferences are tenuous without applicable data on transient jets. Thus, the recommendations in this report are subject to revision if more experience with transient systems is acquired, such as through tests conducted in the separate M3 task.

\subsection{Non-Ideal Mixing}

Simple conceptual models of non-ideal mixing are postulated in Section A.3. They are intended to be used as a basis for correcting, if necessary, ideal mass balance models implicit in the PTF and PEP design bases. However, these are speculative before examining both the uniformity of concentrations over a tank volume and compliance of the mass data to ideal mass balances. As is noted in Section 6.4, the form of any deviations from the ideal need to be inferred from the data itself before expressing it as a model (e.g., hydrodynamic) to be used as a correction to ideal mass balances. Therefore, the means of testing for ideal mixing or correcting ideal mass balances to address non-ideal mixing are themselves subject to interpretation of the data; i.e., inevitably, we will learn from the data how best to interpret the data.

\subsection{Sparger Mixing}

Air spargers are deployed in the UFP-2 vessels to supplement PJMs, creating a "hybrid" mixing system, as is discussed in detail in Appendix B. The derivation of expressions for sparge bubble mixing power, calculations guiding the distribution of spargers in the vessels, and a specific example describing the scaling of sparger design and operation from the WTP- to PEP-scale UFP-2 vessels is provided in Appendix B.

The sparger "mixing effectiveness" is a product of the "intensity of mixing" and the distribution of this "mixing energy" across the slurry contained in the vessel. A suitable distribution is predicted from empirical relationships for the "region of bubbles" (ROB) and "zone of influence" (ZOI) of the bubbles 
on the fluid through which they rise. These are arranged to agitate the fluid over the horizontal extent of the upper region of slurry in a tank while also providing regions for downward "return" flow to enable vertical circulation.

The effect of sparging on mixing and the effect of that mixing on the uniformity of suspended solids is extremely complex. As noted in Section 4.1, it is important to accept that not all predictions of PTF performance can be based on similitude between the PEP and PTF. Based on the analysis in Appendix B, the spargers in the PEP will be arranged and operated to attempt create mixing commensurate with that in the PTF. However, quantitative relationships are not available to infer aspects, if any, of non-uniform mixing observed in the PEP caused by insufficient sparging. Consequently, while every effort has been made to make sparging in the PEP representative of that in the PTF, scaling relationships are not available to propose specific variations of sparging attributes (e.g., air flow rate) in test plans. Rather, the same sparging is proposed for all tests so as not to confound the results by varying attributes for which we cannot propose scaling relationships. 


\subsection{Rationale for Size of PEP}

The concept of a characteristic length serving as the basis for establishing similitude between systems is discussed in Appendix A, Section A.1.4.2. The PEP is designed with a characteristic length $L_{P E P}$ to be $1 / 4.5$ of the characteristic length $L_{P T F}$ of the PTF. Following is the rationale for this size.

\subsection{Practical Considerations Determining the Approximate Size}

Practical lower limits on the size possible for the PEP will still meet the objective of demonstrating an integrated process. The characteristic hydrodynamic time - i.e., the time to fill, empty, or "turn over" a tank or for fluid to "ride" a certain distance along a jet is proportional to the ratio of length scale to velocity, while the time to leach solids is essentially independent of the length scale.

Flows in the PTF on the scale of the equipment are fully turbulent; hence, flows in the PEP must be fully turbulent to represent conditions in the PTF affecting its performance. While this criterion does not require matching Reynolds numbers, it does require sufficiently large Reynolds numbers in the PEP to ensure full turbulence. The Reynolds number is proportional to the product of the length scale and the characteristic velocity. Hence, the characteristic hydrodynamic time is proportional to the square of the length scale at the limit of maintaining fully turbulent flow. For the PEP length scale, this corresponds to hydrodynamic times that are $1 / 20$ of those in the PTF and hence are $1 / 20$ of fixed times such as the time to leach a specified fraction of the Boehmite. Because such ratios differ by more than an order of magnitude, better knowledge of the factors affecting similitude is required to compensate for the disparity in similitude.

As shown in Appendix A, Section A.3.4, the uniformity of suspended solids is affected by the ratio of the settling velocity to the imposed hydrodynamic velocity scale, while the settling velocity does not depend on the length scale. As is shown in Appendix A, Section A.4.3.5, when the slurry exhibits a shear strength, the tendency to form a mixing cavern and the size and shape of any cavern is affected by the yield Reynolds number, which depends on velocity but not the length scale. Hence, there are advantages to matching the velocity in making certain key behavior in the PEP represents that in the PTF. At the same velocity, the Reynolds number is proportional to the length scale. To ensure fully turbulent flow, and more specifically to ensure flow that is as turbulent in the PEP as in the PTF, implies making the PEP not much smaller than the PTF.

The rate of heat loss from the equipment is roughly proportional to the square of the length scale for a specified temperature in a tank. The rate of condensate addition resulting from adding steam to maintain the temperature also is proportional to the square of the length scale, and this continues during caustic leaching. The time for caustic leaching does not depend on the length scale. Therefore, the mass of steam added during leaching is proportional to the square of the length scale, while the mass of slurry being diluted is proportional to the cube of the length scale. Therefore, the ratio of mass of condensate added to mass of slurry diluted is inversely proportional to the length scale. One can compensate for this by decreasing the heat loss using insulation, but the resistance to the heat loss depends on natural convection and radiation heat transfer around the exterior of the equipment, which itself depends on the length scale as well as the detailed configuration of the array of equipment. Thus, while adjusting the heat loss through insulation might alleviate some of the disparity in similitude from dilution by condensation, calculating how to adjust it is difficult, and at best the PEP would not be fully 
representative of the PTF and would require some interpretation whether or not insulation is adjusted. Therefore, regarding the problem of dilution by condensation, it is best to invoke only a moderately small length scale for the PTF.

Finally, maintaining geometric similarity between PEP and PTF equipment becomes difficult because the length scale is so small that commercial equipment or standard fabrication methods cannot be used to create the geometrically similar small system.

The cost of the PEP increases dramatically with its length scale, invoking a cost-benefit analysis based on the effect of length scale described above. During workshops, industrial and other topical experts considered the following:

- Mixing in the ultra-filtration permeate and other vessels was evaluated using scale factors of 4.3 and 4.9 by Meyer et al. ${ }^{1}$ Sparger mixing was simulated in these vessels, as described by Poloski et al. ${ }^{2}$ The staff that performed these tests felt that these scales were approaching the practical limit for sparger-based mixing evaluations.

- PJMs were tested using a scale factor of nine as part of the basis for demonstrating the scalability of PJM-induced mixing by Meyer et al. However, these tests were difficult to perform because of the very short PJM pulse duration.

In summary, technical concerns cause one to design the PEP as large as possible, while constraints on cost require it to be as small as possible. Experts determined that a reasonable compromise is to make the PEP on the order of $1 / 5$ the size of the PTF.

\subsection{Rationale for Exact Size of PEP}

Ultimately, the exact size of the PEP was set to enable both interpreting the evolution of the filtration flux with time and observing any effect of tank mixing on the filter performance. This was accomplished by considering two cases, as follows.

Case I: To match the filtration time constant between the PEP and WTP so the evolution of the filter flux occurs over the same time in the PEP as in the WTP and the ratio of filter fluxes remains close to unity, the ratio of filtration areas must be the cube of the ratio of the characteristic lengths.

Case II: To match this ratio of filtration time to residence time in the PEP to the ratio in the WTP, i.e., to match between the PEP and WTP the ratio of the volume of liquid circulated through the tank to the volume of water removed through the filter, the ratio of filtration areas must be the square of the characteristic lengths if circulation flow velocities entering vessels are matched between the systems as planned.

\footnotetext{
${ }^{1}$ Meyer PA, DE Kurath, and CW Stewart. 2005. Overview of the Pulse Jet Mixer Non-Newtonian Scaled Test Program. PNWD-3677. Battelle-Pacific Northwest Division, Richland, Washington. (WTP-RPT-127)

${ }^{2}$ Poloski AP, ST Arm, JA Bamberger, B Barnett, R Brown, BJ Cook, CW Enderlin, MS Fountain, M Friedrich, BG Fritz, RP Mueller, F Nigl, Y Onishi, LA Schienbein, LA Snow, S Tzemos, M White, and JA Vucelick. 2005. Technical Basis for Scaling of Air Sparging Systems for Mixing in Non-Newtonian Slurries. PNWD-3541. Battelle_Pacific Northwest Division, Richland, Washington. (WTP-RPT-129)
} 
These cases illustrate the need to decide which aspect of similitude is to be established by choosing the characteristic velocity (discussed in detail in Appendix A, Section A 1.3.3).

The ratio of filter areas $A_{F}$ between Case II and Case I is

$$
\frac{A_{F, I I}}{A_{F, I}}=L_{P T F} / L_{P E P}
$$

If the same number of tubes is used for Case I and Case II, the total tube length over the bundles must be in this ratio. Because the available bundles and associated tube lengths are determined from other objectives, the maximum ratio of length scales is also determined. The available bundles are three with 10 -foot tubes and two with 8 -foot tubes. Therefore, the maximum length scale ratio is

$$
\frac{L_{W T P}}{L_{P E P}}=\frac{3(10)+2(8)}{1(10)}=4.58
$$

An integer number of filter tubes must also be employed. To match the flow velocities entering vessels, the volumetric flow rate must be in proportion to the square of the length scale (i.e., the square of the entrance nozzle diameter). Because both the cross-flow velocity and tube diameters are matched between systems, for a scale factor of 4.58 , the number of filter tubes in each filter in the PEP would be the number in the PTF divided by the square of the length scale: $241 /(4.58 * 4.58)$ or 11.49 filters. The closest integer no smaller than 11.49 is 12 filter membranes for the PEP, corresponding to the scale factor 4.5.

The concept of a characteristic length as the basis for establishing similitude between systems is discussed in Appendix A, Section A.1.4.2. The PEP is designed with a characteristic length $L_{P E P}$ to be $1 / 4.5$ of the characteristic length $L_{P T F}$ of the PTF. The rationale for this size is described below.

Case I: To match the filtration time constant between the PEP and WTP so the evolution of the filter flux occurs over the same time in the PEP as in the WTP and the ratio of filter fluxes remains close to unity, the ratio of filtration areas must be the cube of the ratio of the characteristic lengths.

Case II: To match this ratio of filtration time to residence time in the PEP to that ratio in the WTP, i.e., to match between the PEP and WTP the ratio of the volume of liquid circulated through the tank to the volume of water removed through the filter, the ratio of filtration areas must be the square of the characteristic lengths.

These cases illustrate the need to decide which aspect of similitude is to be established by choosing the characteristic velocity (discussed in detail in Appendix A, Section A.2.3.3).

The ratio of filter areas $A_{F}$ between Case II and Case I is

$$
\frac{A_{F, I I}}{A_{F, I}}=L_{P T F} / L_{P E P}
$$


If the same number of tubes is used for Case I and Case II, the total tube length over the bundles must be in this ratio. Because the available bundles and associated tube lengths are decided to meet other objectives, the maximum ratio of length scales is also decided. The available bundles are three with 10 -foot tubes and two with 8 -foot tubes. Therefore, the maximum length scale ratio is

$$
\frac{L_{W T P}}{L_{P E P}}=\frac{3(10)+2(8)}{1(10)}=4.58
$$

An integer number of filter tubes must also be employed. For a scale factor of 4.58 , the number of filter tubes in each filter would be $241 /(4.58 * 4.58)$ or 11.49 filters. Rounding this to 12 filter membranes for the PEP modifies the scale factor to 4.5. 


\subsection{Use of PEP Data}

Data obtained from operating the PEP will be interpreted using the strategy described in Section 2: apply principles of similitude to interpret the data as virtual data from operating the PTF, subject to practical constraints on establishing full similitude between the systems; compare the actual performance of the PEP to that predicted from its design basis to confirm the design basis; and equate the design basis of the PEP and PTF based on comparing the chemical and physical processes that control their performance.

\subsection{Expected Data Set}

PEP operations will obtain

- samples of the slurry at multiple locations and times in the main tanks

- measurements of temperatures at multiple locations and times in the main tanks

- chemical and physical analyses of the samples, including the fraction of solids, composition of the solids, and the composition of the liquid

- measurements of flow rates and volumes transferred at the inlets and outlets of mass balance control volumes, which include the main tanks and filtrate collection.

These elementary measurements will be analyzed to identify and describe the actual process events that resulted in the data, as follows.

\subsection{Data Analysis}

Analysis of the PEP measurements will include

- event mass balances associated with transfers of slurry between tanks, which provide specifically

- masses transferred between tanks

- masses filtered

- masses contained in tanks, including

- solid fractions in slurries

○ composition of solids

$\circ$ composition of liquids

- cumulative mass balances over multiple events or over time, which provide

- estimates of any accumulation of settled solids

- estimates of mass balance measurement uncertainties

- statistical tests and uncertainty analysis of uniformity and of fidelity to mass balances when applicable to the data.

These analyses create a history of the actual evolution of the contents of process tanks that can be compared to that predicted from the design basis combined with logs of the operation. 


\subsection{Inferences}

From the PEP data can be inferred

a. the evolution of filtration rate over time under conditions expected in the PTF (see Section 3.7.3); this directly meets one of the objectives of the PEP

b. the uniformity of concentrations, temperatures over the slurry volume in the main tanks, which is the foundation of design basis mass balances (see Section 5.1 and Appendix A, Section A.2)

c. the validity of design basis predictions of wash and leach factors (see Sections 5.3 and 5.4) made using mass balances for which uniform concentrations and temperatures are presumed.

Then

- inference (a) predicts the expected performance of the PTF, using principles of similitude

- inference (b) tests the fundamental assumption in the design basis that the composition of streams exiting a tank is the average composition in a tank (see Section 3.3) and that point measurements of temperature acceptably represent that averaged over the volume of a tank during leaching

- inference (c) confirms the design basis used to predict the expected performance of washing and leaching the PTF.

\subsection{Correcting Mass Balances}

Specific conceptual models of systematically non-uniform distribution of solids are proposed in Appendix A, Section A.2.1.2. These models provide trial forms for the function " $F$ " presented in Section 5.1, i.e.,

$$
\phi_{S, \text { out }}=f\left(\overline{\phi_{S}}, \phi_{S, i n}, \text { flow field }\right) \text { and } C_{j, \text { out }}=f\left(\overline{C_{j}}, C_{j, \text { in }}, \text { flow field, chemical reactions }\right)
$$

that can be tested using statistical tests of fidelity to mass balances identified in the previous section. Actual corrections to the mass balance may be of this form if found to explain any discrepancies in the mass balances, but in any event will be determined by the data itself. 


\subsection{Technical Guidance for PEP Test Planning}

\subsection{Summary}

The PEP has been designed and is being constructed. Thus, the size (length scale) is set, but the time scale (imposed velocities and durations of process steps) and thermal and chemical attributes can be set to best obtain data from which to meet the objectives of the PEP. The purpose of this section is to provide technical guidance supporting the development and evolution of test plans for the PEP. Essentially, this guidance is based on a combination of the principles presented in Sections 2.1 and 2.2; i.e., either

- interpreting PEP data to predict the performance of the PTF based on similitude - essentially extrapolating PEP data to the length and time scale of the PTF to predict certain aspects of its performance-or

- comparing PEP performance with that predicted from the design principles and process data to demonstrate the adequacy of (or correct) those principles and process data, and then apply the same or corrected principles to predict the performance of the PTF.

These two complementary philosophies - extrapolation via similitude and demonstrating the technical basis for the PTF design - can lead to alternative technical guidance. In the first case, the objective is to discover the behavior that will occur in the PTF by establishing representative conditions in the PEP. In the second case, the objective is to discover any inadequacies in the PEP design by operating it under conservative conditions; i.e., under conditions more challenging in the PEP than are actually expected in the PTF so that adequate performance of the PEP provides confidence in the robustness of the design principles and process data used to design the PTF.

The resulting alternatives can be mutually exclusive, in which case one must choose between establishing representative conditions or conservative conditions. Where it is possible to establish similitude for some specific performance attribute, this is a compelling choice. But as is noted in Section 4.1,

“... while it is important to understand and optimally apply principles of similitude, it is equally important to understand and accept that no set of adjustable parameters establishes full similitude between the UFP in the PEP and the PTF and therefore accept that not all predictions of PTF performance can be based on similitude between the PEP and PTF. Consequently, we must optimize what can be deduced from similitude and dimensional analysis and (mainly) apply it in terms of the simplest relationships between PTF performance and PEP data."

The final point, applying "in terms of the simplest relationships between PTF performance and PEP data," invokes the need to control variations over interacting variables that otherwise confound interpretation of performance data. In particular, as is noted in Section 3.2.3,

"Because interactions between physical and chemical attributes depend on both temperature and concentration, they are exceedingly complex. The only practical choice to provide thermal and chemical similitude is to match the thermal and chemical scales between systems." Matching "chemical scales" means using the same simulated waste and the same process reagents in the PEP as will be used in the 
PTF. Matching "thermal scales" means imposing the same temperatures at corresponding times during corresponding steps during the process. The complex and strong interactions among mass balance, kinematic, hydrodynamic, chemical, and thermal processes preclude establishing these correspondences through similitude. Therefore, in some cases it is necessary to directly impose the desired temperature sequences through direct control by steam injection and active heat exchange. In other cases it may be possible to match the thermal scale by adjusting insulation to adjust heat transfer coefficients in the PEP to differ as necessary from those in the PTF.

Given this, where it is difficult to establish similitude, we must rely on a conservative vindication of design principles and process data to validate the technical basis for designing the PTF and thereby validating predictions of its performance attending the design calculations.

The actual operating sequences may be different than as might be inferred here, such as utilizing upfront leaching in vessel UFP-1 instead of nominally in UFP-2 as is implied here. The operations implicit in the following recommendations serve only to define the phenomenal and variables relevant to the significance of scaling issues to test schemes and do not constitute recommendations about process alternatives.

This section is divided into seven subsections, each providing technical guidance for planning operation of the PEP for the seven corresponding process functions discussed in Section 3: dewatering, washing, adding reagents, heating, caustic leaching, cooling, and oxidative leaching (effects of length and time scale common to all process functions are described in Section 3.2). The purpose of the functions is to provide data from which to quantify the performance of the PTF in terms of the same functions. The following subsections describe phenomena controlling the performance of the process function, effects of time scale specific to the function, and specific technical guidance, as summarized in Table 7.1. The rationale underlying the guidance are discussed in each subsection.

Table 7.1. Summary of Technical Guidance

\begin{tabular}{|c|c|c|c|}
\hline Process Function & Important Phenomena & Time Scale Parameters & $\begin{array}{l}\text { Technical Bases for Test } \\
\text { Planning }\end{array}$ \\
\hline Dewatering & $\begin{array}{l}\text { - Filtration } \\
\text { - Mixing } \\
\text { - Blending }\end{array}$ & $\begin{array}{l}\text { - Ratio of filtrate volume to } \\
\text { solids mass filtered } \\
\text { - PJM nozzle velocity } \\
\text { - Ratio of PJM mixing to } \\
\text { mixing by circulation }\end{array}$ & $\begin{array}{l}\text { Similitude in filtration } \\
\text { performance: } \\
\text { - Match solids mass/filter } \\
\text { area } \\
\text { Conservative test of design } \\
\text { assumptions: } \\
\text { - Match Yield Reynolds } \\
\text { number }\end{array}$ \\
\hline Washing & $\begin{array}{l}\text { - Mixing } \\
\text { - Suspension of solids } \\
\text { - Chemical reaction } \\
\text { - Filtration }\end{array}$ & $\begin{array}{l}\text { - Ratio of PJM velocity to } \\
\text { settling velocity } \\
\text { - Yield Reynolds number } \\
\text { - Froude number } \\
\text { - Reynolds number }\end{array}$ & $\begin{array}{l}\text { Similitude in washing } \\
\text { phenomena: } \\
\text { - Match yield Reynolds } \\
\text { number } \\
\text { Conservative test of design } \\
\text { assumptions: } \\
\text { - Match Froude Number }\end{array}$ \\
\hline Adding Reagents & $\begin{array}{l}\text { - Local blending in pipe flow } \\
\text { - Turbulence in filter loop }\end{array}$ & $\begin{array}{l}\text { - Local blending time } \\
\text { - Tank blending time }\end{array}$ & $\begin{array}{l}\text { Similitude: } \\
\text { - Match ratio of reagent } \\
\text { injection rate to pipe flow }\end{array}$ \\
\hline
\end{tabular}

\footnotetext{
${ }^{1}$ in case non-Newtonian behavior decreases mixing and affects solids loading in filtration feed
} 
Table 7.1. (contd)

\begin{tabular}{|c|c|c|c|}
\hline Process Function & Important Phenomena & Time Scale Parameters & $\begin{array}{l}\text { Technical Bases for Test } \\
\text { Planning }\end{array}$ \\
\hline Heating & $\begin{array}{l}\text { - Heat balance } \\
\text { Thermo-physical properties } \\
\text { - Chemical reaction during heating }\end{array}$ & $\begin{array}{l}\text { - Ratio of steam injection } \\
\text { rate to tank mass } \\
\text { - Ratio of steam injection } \\
\text { rate to heat transfer losses }\end{array}$ & $\begin{array}{l}\text { Direct Control } \\
\text { - Match temperature versus } \\
\text { time }\end{array}$ \\
\hline Caustic Leaching & $\begin{array}{l}\text { - Chemical Reaction } \\
\text { - Mixing }\end{array}$ & $\begin{array}{l}\text { - Chemical reaction rate } \\
\text { - Ratio of mixing time to } \\
\text { reaction time } \\
\text { - Ratio of PJM velocity to } \\
\text { settling velocity }\end{array}$ & $\begin{array}{l}\text { Direct Control: } \\
\text { - Match reaction time } \\
\text { - Match either power/volume } \\
\text { or Yield Reynolds Number }\end{array}$ \\
\hline Cooling & $\begin{array}{l}\text { - Heat balance } \\
\text { - Thermo-physical properties } \\
\text { - Chemical reaction during cooling, } \\
\text { including precipitation reactions }\end{array}$ & $\begin{array}{l}\text { - Ratio of heat transferred to } \\
\text { mass in tank }\end{array}$ & $\begin{array}{l}\text { Direct Control } \\
\text { - Match cooling time }\end{array}$ \\
\hline Oxidative Leaching & $\begin{array}{l}\text { - Chemical reaction } \\
\text { - Mixing }\end{array}$ & $\begin{array}{l}\text { - Chemical reaction rate } \\
\text { - Ratio of mixing time to } \\
\text { reaction time } \\
\text { - Ratio of PJM velocity to } \\
\text { settling velocity }\end{array}$ & $\begin{array}{l}\text { Direct Control: } \\
\text { - Match reaction time } \\
\text { - Match either power/volume } \\
\text { or Yield Reynolds Number }\end{array}$ \\
\hline
\end{tabular}

\subsection{Dewatering}

Dewatering removes liquid from the slurry to increase the concentration of solids. Liquid is removed by filtration as slurry is fed to UFP-2. Because filtration occurs as the solids concentration increases, and filter performance is challenged by the increasing concentration of solids, the relationship between filter flux and solids concentration is the principal technical issue and basis for the design of dewatering tests in the PEP.

\subsubsection{Controlling Phenomena}

During dewatering, the filter flux and solids concentration are coupled through a mass balance over the tank and filter; the mass balance depends on the relationship between the mass of solids in the tank and the concentration in its effluent flowing to the filter, which is a consequence of ideal mixing in the tank. Therefore, the phenomena controlling the performance of the PTF and PEP during dewatering substantially affect both the filter flux and mixing in the filter feed tank.

\subsubsection{Filtration}

Because dewatering removes liquid from the vessels, the controlling phenomenon is filtration. The filter tubes will be identical between the PEP and PTF; the resulting lack of geometric similitude is accepted and intentional. The filter flux is affected by the viscosity of the liquid phase, which is affected by temperature, the concentration of solids in the slurry, and parameters to be matched between the PEP and PTF: the trans-membrane pressure and cross-flow velocity. Fine solids accumulate within the filter media during filtration and decrease filter flux (depth fouling), and as the solids concentration increases, eventually a filter cake can form on the surface of the filter media further decreasing filter flux. Thus, the evolution over time of the filter flux depends on the evolution over time of the liquid composition and solids concentration in the slurry fed to it. 


\subsubsection{Mixing}

Maintaining a uniform concentration is incidental to the dewatering function per se; i.e., the measure of performance, per se, is only water removed. However, accumulation of solids at the level of the filter loop inlet detrimentally increases the solids concentration fed to the filter and also uniformity affects the validity of the mass balance on which interpretation of the filter flux evolution is based. Therefore, it is important to keep solids suspended uniformly during any process step involving filtration.

The evolution over time of the slurry attributes during filtration is determined by the conservation of solids mass in the feed tank and the filter during filtration. Mass balances used as the design basis, and hence as the means of interpreting process data to control the process, are based on the assumption that the exiting solids concentration equals the average concentration in the tank, which is the mass of solids in the tank divided by the volume of slurry in the tank. See Section 7.3.1.2 for a discussion of Mixing.

\subsubsection{Blending}

Blending denotes the mixing of mass entering a vessel with the mass in the vessel as slurry flows through the tank. To distinguish this from mixing, blending denotes processes of perfect mixing. If the inlet concentration remains constant, eventually the outlet concentration and average concentration in the tank will equal the inlet concentration. However, if the inlet concentration changes continuously, as occurs during dewatering, the outlet and inlet concentrations will always differ by an amount that depends on the rate of change of the inlet concentration compared to the residence time in the vessel; that is, it depends on the ratio of two characteristic times. For filtration, these are the residence time in the tank, which is the slurry volume divided by the volumetric flow rate in the filter loop, and the time to filter the tank volume, which is the slurry volume divided by the volumetric flow rate of filtrate through the filter.

\subsubsection{Effect of Time Scale}

Several time scales are pertinent to dewatering: the circulation residence time in the feed vessel, $t_{C}$, the time to filter the vessel volume, $t_{F}$, the PJM cycle time, $t_{J}$, and the PJM drive time, $t_{D}$. The time $t_{C}$ is set by the recirculation rate; the time $t_{F}$ is set by the filter area for a given filter flux.

The velocities pertinent to mixing during dewatering are velocity at the filter loop inlet to the tank and velocity at the PJM nozzles. The filter loop nozzle jet velocity is determined by the filter loop volumetric flow rate and the nozzle diameter. The PJM velocity is transient; its time-average is determined by both the cycle time $t_{J}$ and the drive time $t_{D}$ of the PJM, the volume of the PJM pulse, and the size of the PJM nozzle. The resulting nozzle velocity during the pulse, $u_{J}$, is proportional to the volume of the pulse divided by the product of the nozzle area and the drive time, which is proportional to the length scale divided by the drive time. The nozzle velocity time-averaged over the cycle, $u_{J, a v e}$ is the product of transient velocity and the ratio $t_{D} / t_{J}$, which is proportional to the volume of the pulse divided by the product of the nozzle area and the cycle time, which is proportional to the length scale divided by the cycle time.

Transient flows cannot be described by a single parameter such as a constant velocity. Because the PTF and PEP tanks are very complex flow situations, we cannot determine a priori some means of reducing, say, the velocity-time relation at the PJM nozzle to a single attribute on which to base some performance measure such as uniformity of mixing or fraction of solids remaining settled. Although the PJM nozzle velocity averaged over the cycle might suffice, until PEP data are available to analyze, we assume the transient characteristics also could be important. In that case, one would want to match both 
the transient and the average PJM nozzle velocities in the PEP to those in the PTF by matching the product of the drive time and the cycle time and the length scale. We can vary the transient or average PJM nozzle velocity independently of the circulation nozzle velocity to invoke any overall effect on the dewatering process.

The filter flux is a test result that will depend on the choice of test parameters, which can include the filter area. Therefore, the test choices affecting the pertinent time scales are the recirculation rate and the filter area. However, during filtration the cross-flow velocity is proportional to the circulation rate, and the cross-flow velocity is matched between systems. Therefore, the available test choices affecting the ratio of pertinent time scales are the filter area and the PJM drive time.

\subsubsection{Technical Guidance}

As discussed in the previous section, the available choices of test parameters affecting dewatering are the filter area and the PJM drive time. As discussed in Section 5, the length scale of the PEP was set to either match the filtration time scale of the PEP to that in the PTF or to match the filtration time scale in the PEP to the feed vessel residence time scale in the PEP. Matching the filtration times causes the filter flux to evolve over time in the PEP as in the PTF, except for any effect of the relative time scales for filtration and blending in the feed tank. Matching the filtration time scale to the vessel residence time matches the time scales of filtration and blending, but as shown in Appendix A, Section A.2.3, the evolution of the filter flux depends on the ratio of the filter area to the initial mass of solids. The effect of not matching the area/mass ratio is accounted for in part by comparing fluxes as a function of volume of filtrate passed instead of time elapsed, but the area/mass ratio also can affect the evolution of the flux as a function of filtrate volume passed. Therefore, not matching the ratio of filter area to initial solids mass introduces difficulties in interpreting the filter flux but allows examination of possible effects of the rate of change of solids concentration in the vessel, which is determined by the filter area, to the residence time in the vessel.

The two available choices of the filter area are operating with one filter bundle in the loop and with all five filter bundles in the loop. As noted above, the former choice matches the filtration time between the PEP and the PTF, while the latter matches the filtration time to the residence time in the feed vessel if the velocity also is matched at the circulation inlet nozzle in the tank. The former provides similitude in the form of an evolution of the filter flux due to loading with fines or filter cake, which represents the evolution in the PTF, if mixing effects in the feeds vessel are unimportant, such as if the mixing is more than adequate to maintain a uniform solids concentration in the tank. Also, the latter choice allows collecting filtrate over several lengths along the sequence of filter tubes in series, which allows determining the axial uniformity of the filter flux as the filters load with fines or with filter cake.

Therefore, scaling arguments suggest that the PEP should be operated primarily using a single filter bundle to match the ratio of filter area to solids mass. However, secondarily the PEP should also be operated using all filter bundles to 1) enable the filter flux over several bundles in series to be compared to the flux occurring in the first bundle, from which one can estimate the variation of flux with downstream distance caused by axially non-uniform profiles in filter plugging or formation of filter cake, and 2) determine if the rate of blending in the filter feed tank-i.e., the ratio of the "turnover" time to the time to filter the tank contents - affects the solids concentration in the filter feed sufficiently to affect the filter flux. 


\subsection{Washing}

\subsubsection{Controlling Phenomena}

Washing is simultaneous addition of (essentially) water, thorough mixing of suspended solids with the liquid phase, and removal of liquid by filtration. The controlling phenomena are bulk mixing of the liquid phase over the volume of the tank, suspension of solids in the liquid, chemical equilibrium between solids and the liquid, and filtration.

Ideal washing is defined here to be the washing achieved if each incremental addition of wash water (condensate) were instantaneously mixed throughout the entire slurry. Thus, ideal washing requires ideal mixing: either stagnant regions or particle settling thwart ideal washing. Given ideal mixing, any soluble particles dissolve as the otherwise saturated surrounding liquid is diluted by the condensate.

\subsubsection{Filtration}

Because, averaged over time, the liquid is replaced approximately as fast as liquid is removed by filtration, filtration is the principle phenomenon controlling the rate of washing. The technical issues are discussed in Section 7.2.1.1.

\subsubsection{Mixing}

Mixing is a complex set of physical processes; herein terms such as "mixing," "mixed," "good mixing," or "well mixed" denote the extent to which the mass of solids in a vessel equals the product of the slurry volume and the concentration of solids exiting the vessel.

Imperfect mixing can include settling of solids, stagnant regions of slurry, and a "short circuit," denoting that part of the entering flow proceeds to the outlet without mixing at all with the contents of the vessel.

Mixing in the vessels is determined by the properties of the slurry, which ostensibly are matched between systems, and by the velocities imposed on the slurry. Velocities are imposed at the filter loop inlet to the tank and at the PJM nozzles.

\subsubsection{Suspension of Solids}

One potential cause of poor mixing is solids settling - the accumulation of solids on the floor of a vessel. During washing, the slurry probably will be non-Newtonian, exhibiting a yield stress that could obviate settling. For sufficiently large and dense particles, settling is still possible. The average concentration of solids in a tank is the total solids mass - including settled solids - divided by the slurry volume. The exiting concentration is the suspended mass divided by the slurry volume. Hence, any settling could cause the concentration of solids exiting a vessel to be less than the average concentration in the vessel.

As discussed in Appendix A, Section A.3.2.1, in steady-state systems not dominated by nonNewtonian behavior (that is, the less likely case), the concentration of a slurry with given particle attributes that can be kept suspended has been found empirically to be a function of the product of the velocity imposed and the length scale to approximately the $1 / 6$ power (i.e., an exponent of about $1 / 6$ ). 
We do not have a firm basis to conclude the same functional form applies to suspension by transient jets, yet currently we have no better premise available from the engineering literature as a basis for anticipating the ability to suspend solids in the PEP or PTF. Industrial experts advise that equal power/volume often is relied on to scale-up mixers to maintain adequate solids suspension and uniformity of concentration.

\subsubsection{Mixing of Suspended Solids}

Solids that are kept suspended may or may not form a vertically uniform concentration under the influence of gravity. Two cases of adverse processes can potentially prevent solids from mixing uniformly. First, if particles settle fast enough, there is a competition between the settling velocity that is everywhere downward and the dispersion of particles by turbulence (see Appendix A, Section A.3.1). Second, even if particles do not settle rapidly, transient horizontal density gradients caused by jet flow into a vertically non-uniform solids concentration can impede the penetration of PJM jet flows into the upper part of tanks, thereby perpetuating the impeding density difference (see Appendix A, Section A.3.3). We expect the first condition, if in fact important, to be represented correctly in the PEP if we match hydrodynamic velocities between the PEP and PTF. As discussed in Appendix A, Section A.3.3, application of a simple conceptual model and a similitude analysis based on work done in lifting solids suggests that the potential for the second case to occur in the PEP is represented in the PEP by matching $u_{J}^{3} t_{D} / L^{2}$, where $u_{J}$ is the PJM nozzle velocity, $t_{D}$ is the PJM drive time, and $L$ is the length scale.

\subsubsection{Chemical Reaction}

Washing includes chemical reactions in that dissolution of a soluble solid is a chemical reaction between the solid and (in this case, essentially) water to form the dissolved species in water. This reaction is often fast, but generally the rate decreases as the solubility decreases, and where the dissolution rate potentially limits the overall rate of washing, it defines a characteristic time to be matched in ratio to other, e.g., hydrodynamic, time constants. We assume here that dissolution occurs fast enough that the ratio of hydrodynamic times to "chemical" times during washing has no effect on wash factors.

\subsubsection{Effect of Time Scale}

In the likely case of washing solids in a non-Newtonian slurry, the principal concern is the formation of a mixing cavern surrounded by stagnant slurry. Condensate added to accomplish the washing would be poorly distributed to the stagnant region. As is discussed in Appendix A, Section A.4.3.5, we expect the occurrence of mixing caverns to depend on the yield Reynolds number. That is, we need to match the yield Reynolds number to provide similitude specific to this phenomenon. Given the same fluid properties, matching the yield Reynolds number requires matching the velocities. That is, the yield Reynolds numbers scales as $\mathrm{uL}^{0}$.

In the event the slurry behaves as a Newtonian fluid during washing, to match the ability to suspend solids - i.e., the ability to prevent solids from accumulating on the floor of a vessel—we match the product $u L^{-1 / 6}$ or $u L^{-1 / 3}$ between the PEP and the PTF. As discussed in Appendix A, Section A.3.1, if the settling velocity can cause concentration gradients, we expect the uniformity of suspended solids to "scale" as the ratio of the particle settling velocity to the characteristic velocity imposed on the system. Then, as for the yield Reynolds number, because the settling velocity is a property of the slurry and 
because such properties will be matched between the PEP and the PTF, we expect the ability to maintain a uniform solids concentration will be matched by matching the velocities imposed in the PEP and the PTF. That is, we would match $\mathrm{uL}^{0}$.

If transient horizontal density gradients impeding the penetration of PJM jet flows into the upper region of tanks is an issue, the phenomenon is represented by matching the term $u_{J}^{3} t_{D} / L^{2}$ between the PEP and PTF (see Section 7.3.1.4). Given geometric similarity and relating the drive time to the nozzle velocity, this relationship is equivalent to matching $\mathrm{uL}^{-1 / 2}$, corresponding to matching the densimetric Froude number.

Industrial experts advise that the power/volume ratio is often used as a criterion for good mixing and suspension of solids in industrial blenders and stirred-tank reactors. This requires matching the product $u L^{-1 / 3}$ between the PEP and the PTF.

Finally, the relative intensity of turbulence - the ratio of the magnitude of turbulent fluctuations in velocity to the time-averaged velocity - is determined by the Reynolds number, albeit not necessarily strongly. The Reynolds number is matched between systems by matching the product $u L^{1}$.

\subsubsection{Technical Guidance}

Based on the above, possible "scale-up" options related to mixing include matching any of the products $u L^{1}, u L^{0}, u L^{-1 / 6}, u L^{-1 / 3}$, or $\mathrm{uL}^{-1 / 2}$. Matching $u L^{0}$ provides a representative ratio of imposed velocity to settling velocities and provides a representative yield Reynolds number, which describes the tendency for stagnant regions to form at the top of a vessel when the slurry displays a shear strength, as is plausible for concentrated slurries. It also matches the ratio of the velocity to the settling velocity. Anticipating the solids will be washed in non-Newtonian slurries, matching the yield Reynolds number is the first priority, meaning matching $\mathrm{uL}^{0}$ (matching velocities).

Matching $u L^{-1 / 6}$ or $u L^{-1 / 3}$ ought to represent in the PEP the same tendency to suspend solids as in the PTF. Experts advise that matching $u L^{1 / 3}$ (power/volume) ought to represent the same tendency both to suspend solids and establish a uniform solids concentration over suspended solids. The benefit of matching the Reynolds number is not established for lack of a clear link between the relative intensity of turbulence and wash factors. However, it is important for the Reynolds number in the PEP to be large enough to correspond to fully turbulent flow as in the PTF.

Matching $\mathrm{uL}^{-1 / 2}$ ought to represent the effect of bulk density differences-i.e., the persistence of "clouds" over multiple PJM pulses - on suspension of solids. Because a significant fraction of the volume in the UFP tanks is occluded by the PJM mixers, the resulting complex flow patterns might disorganize horizontal density gradients that otherwise might persist due to impeded penetration of PJM jets. If so, jets would tend to penetrate the tank volume, probably making the potential problem unimportant and decreasing the benefit of matching $\mathrm{uL}^{-1 / 2}$.

Matching $\mathrm{uL}^{-1 / 2}$ results in the lowest velocity in the PEP. No phenomenon is identified in this report causing the performance of the PEP or PTF to decrease with increasing velocities. Therefore, the least velocity is the most conservative choice. However, as is discussed in Section 7.1, the most conservative 
choice can result in decreased similitude. As is apparent from the above discussion, there are multiple aspects of similitude pertinent to mixing processes affecting washing, or any of the process steps sensitive to mixing.

In the event non-Newtonian behavior — such as cavern formation — does not dominate during washing, matching power/volume $\left(\mathrm{uL}^{-1 / 3}\right)$ as has been recommended by industrial experts, provides a good balance between conservatism and similitude in phenomena affecting suspension and mixing.

When matching power/volume, one could match $\mathrm{uL}^{-1 / 3}$ between systems given some definition of $\mathrm{u}$. However, because PJM mixing is inherently transient, it would be best to match the ratio of the power added per cycle to the volume of slurry being mixed, taking into consideration all parameters, such as volume displaced during a pulse and the drive and cycle times, rather than defining a specific velocity to scale.

In summary, the first priority is to match $\mathrm{uL}^{0}$ — match the velocity, in anticipation of non-Newtonian behavior dominating the mixing process. The second priority is to match power/volume (match this directly, but noting that nominally this is equivalent to matching $\mathrm{uL}^{-1 / 3}$ ) to provide a more conservative case (lesser velocity used in the PEP).

\subsection{Adding Reagents}

Leaching reagents (caustic, permanganate) will be added in the filter loop to accomplish in-line mixing. Reagents are added at a specified volumetric flow rate and mix with the volumetric flow rate of slurry in the pipe of the filter loop. Perfect in-line mixing would be a uniform dilution of the reagent by the slurry as it exits the pipe into the vessel.

\subsubsection{Controlling Phenomena}

The controlling phenomenon is turbulent mixing in the filter loop pipe. Blending of the loop discharge into the tank is also important, but blending in the tank of liquid with comparable properties is less an issue than blending two liquids with differing densities and viscosities, an issue noted by the EFRT. Successful turbulent mixing in the pipe will prevent the properties of the loop effluent from deviating significantly from the tank contents, thereby mitigating the issue.

The effectiveness of mixing in the filter loop pipe is described by the local blending time. The physical parameters affecting in-line mixing include the volumetric flow rate of the slurry and the size, length, fittings, etc. of the filter loop. For example, the caustic is added upstream of two large centrifugal pumps, is blown through five sets of ultrafilters, and goes through a spiral plate heat exchanger, a flow meter, U-turns, etc. These determine the characteristic mixing time between materials traveling together in the pipe, the properties of the two materials being mixed, and the relative volumetric flow rates of the materials.

The transit time for reagents added a certain length upstream of the exit from the pipe is the product of that length and the pipe cross-sectional area divided by the flow rate of the slurry, which includes the flow rate of the reagent being added. Assuming geometric similarity, this time is proportional to the cube of the length scale divided by the volumetric flow rate. Therefore, the ratio of transit time to characteristic mixing time would be the same between systems if the ratio of volumetric flow rate to 
length scaled cube is the same, but for practical reasons they are not. However, the filter loop flow is highly turbulent in both systems, and the ratio of pipe diameters maintains geometric similarity.

The volumetric flow rate is set by setting conditions in the filter tubes through which the slurry circulates. As is discussed in Section 5.2, full-size filter tubes are used in the PEP, and the cross-flow velocity is matched to that in the PTF. The filter cross-sectional flow area is proportional to the number of filter tubes through which the slurry flows in parallel, and the volumetric flow rate through each tube is that in the PTF; hence, the ratio of volumetric flow rates is proportional to the ratio of filter tubes. This ratio is discussed in Section 5.2.

\subsubsection{Technical Guidance}

Combining the information in the previous section, the lack of full geometric similarity in the filter loop piping precludes directly matching the ratio of in-line characteristic mixing time to in-line transit time in the PEP and PTF. However, if the materials are the same and the relative volumetric flow rates of the reagent and slurry are the same, the uniformity of mixing at the filter loop exit into the PEP feed vessel should adequately represent that in the PTF. Therefore, one should match the ratio of reagent volumetric feed rate to filter loop volumetric flow rate between the PEP and the PTF.

\subsection{Heating}

The slurry is raised to the temperature required for caustic leaching by adding live steam.

\subsubsection{Controlling Phenomena}

The controlling phenomena are a heat balance and mixing the locally heated liquid with the rest of the slurry. Mixing is discussed in Sections 7.3.1.2 and 7.3.2.

\subsubsection{Effect of Time Scale}

The transient heat balance has the form

$$
m C_{p} \frac{d T}{d t}=\dot{m}_{\text {steam }} \Delta H^{\text {vap }}-A h\left(T-T_{a}\right)
$$

where $m$ and $C_{P}$ are the mass and specific heat of the slurry being heated, $T$ is the temperature, $T_{a}$ is the temperature of the surroundings, $t$ is time, $\dot{m}_{\text {steam }}$ is the mass rate of addition of steam, $\Delta H^{v a p}$ is the enthalpy change from steam to water dissolved in the slurry, $A$ is the area of transfer of heat to the surroundings, and $h$ is the effective heat transfer coefficient to the surroundings. For a constant rate of addition of steam,

$$
\frac{T-T_{a}-\frac{\dot{m}_{\text {steam }} \Delta H^{\text {vap }}}{A h}}{T_{0}-T_{a}-\frac{\dot{m}_{\text {steam }} \Delta H^{\text {vap }}}{A h}}=e^{-\frac{A h}{m C_{p}} t}
$$

where $T_{0}$ is the initial temperature. Then if some temperature $T_{H}$ has been attained after time $t_{H}$, 


$$
t_{H}=\frac{m C_{p}}{A h} \ln \frac{1-\left(T_{0}-T_{a}\right) \frac{A h}{\dot{m}_{\text {steam }} \Delta H^{\text {vap }}}}{1-\left(T_{H}-T_{a}\right) \frac{A h}{\dot{m}_{\text {steam }} \Delta H^{\text {vap }}}}
$$

For sufficiently large rates of addition of steam, this reduces to

$$
t_{H}=\frac{m C_{p}}{\dot{m}_{\text {steam }} \Delta H^{\text {vap }}}\left(T_{H}-T_{0}\right)
$$

The mass $m$ is proportional to the cube of the length scale, therefore, so is $t_{H}$ for a given rate of addition of steam, or, the rate of addition of steam $\dot{m}_{\text {steam }}$ to attain a temperature in a specified time is proportional to the cube of the length scale. The rate of steam can be adjusted as needed to cause the heating time in the PEP to equal that in the PTF. The rate of heat transfer, which through the area $A$ is proportional to the square of the length scale, does not affect the heating time if the heating rate is great enough to satisfy

$$
\left(T_{H}-T_{a}\right) \frac{A h}{\dot{m}_{\text {steam }} \Delta H^{\text {vap }}}<<1
$$

where $T_{H}$ is the target temperature.

As is discussed in Section 3.4.1, for a given $\Delta T$ being maintained, the rate of accumulation of water is proportional to the area, i.e., to the square of the size of the system. On the other hand, the mass of slurry being diluted by the accumulating water is proportional to the cube of the system. Therefore, the rate of dilution expressed as the fractional mass added per time, i.e., the dilution per time increases in inverse proportion to the size of the system if the overall heat transfer coefficient, $h_{0}$, is the same between systems. Therefore, to minimize the difference between systems of the fractional dilution caused by condensation, some adjustment of the overall heat transfer coefficient or some direct adjustment of the heat transferred is needed. Such adjustments complicate interpretation (e.g., extrapolation to larger scale) of any mal-distribution of temperature seen in the PEP. However, nominally, the temperature distribution in the PEP is expected to be nearly uniform while the effect of dilution by condensation likely could affect the leach rate by changing the concentrations of reagents and reaction products.

As is discussed in Section 2.1, the phenomena controlling the performance of the UFP in the PTF are physically and chemically complex, and we must accept that not all predictions of PTF performance can be based on similitude between the PEP and PTF. In this case, it is more important to control the temperature to enable interpreting leach factors than to maintain similarity in the thermal boundary conditions to enable interpreting the resulting uniformity of temperature during caustic leaching.

An estimate of the extent of dilution-whether it will be similar to that expected in the PTF or inordinately greater - will be estimated during functional testing of the PEP. If found to be necessary, the thermal insulation can be adjusted, and also direct temperature control can be imposed through the use of heat exchangers to decrease the rate of condensation during caustic leaching. 


\subsubsection{Technical Guidance}

Because the conversion during caustic leaching is affected by the entire temperature history after adding caustic, the leach factors observed will be difficult to interpret unless the temperature history is the same between the PEP and PTF. Thus, the temperature in the PEP should increase, remain constant, or decrease as a function of time in the PEP as in the PTF. Consequently, steam should be added to establish the desired relationship of temperature to time. Secondarily, the mixing and agitation provided also should be the same during heating, caustic leaching, and cooling. However, as is noted in Section 7.1, the combined phenomena are too complex to allow using similitude to extrapolate the temperature distributions measured during PEP operation to the PTF.

\subsection{Caustic Leaching}

Caustic leaching comprises a chemical reaction with caustic converting leachable aluminum species to the soluble form $\mathrm{Al}(\mathrm{OH})_{4}{ }^{-}$. The caustic leaching step is described in process models in terms of a leach factor, which is the fraction of the initial mass of the target insoluble solid that is removed from the waste by filtration after dissolution by leaching. This factor is affected by 1) the fraction of the solid converted to the soluble form, 2) the fraction of the soluble form dissolved, and 3) the fraction of the dissolved soluble form removed as filtrate. Consequently, leach factors are a combination of the chemical conversion and the subsequent separation of soluble from insoluble materials.

\subsubsection{Controlling Phenomena}

\subsubsection{Dewatering and Washing}

Although ultimately dewatering and washing affect leach factors, they are treated as separate topics in this report. The phenomena controlling dewatering and washing are discussed in Sections 7.2 and 7.3.

\subsubsection{Chemical Conversion}

Caustic leaching of aluminum oxides is a reversible chemical reaction with a rate determined by the temperature, concentration of the reactant (caustic), and concentration of the reaction product anion, $\mathrm{Al}(\mathrm{OH})_{4}{ }^{-}$. The phenomena controlling the chemical conversion are discussed in detail in Appendix A, Section A.2.2. In summary, the chemical conversion has the approximate functional dependence

$$
X=X\left(t, T(t), C_{\mathrm{OH}^{-}, \text {initial }}, C_{A l(\mathrm{OH})_{4}^{-}, \text {initial }},\left(m_{S}(t) / V\right)\right)
$$

where

$$
\begin{aligned}
X & =\text { the conversion (mass of } \mathrm{AlO}(\mathrm{OH}) \text { converted per initial mass of } \mathrm{AlO}(\mathrm{OH})) \\
T & =\text { time } \\
T(t) & =\text { the temperature history during the leaching step, where } d X / d T>0 \\
& =\text { the concentration of caustic at the start of the leaching step, where }\left(d X / d C_{\mathrm{OH}^{-}}\right)>0 \\
C_{\mathrm{OH}^{-}, \text {initial }} & =\text { the reaction product anion at the start of the leaching step, where }\left(d X / d C_{A l(\mathrm{OH})_{4}^{-}}\right)<0 \\
C_{A L(O H)_{4}^{-}, \text {initial }} & =\text { history of the mass of solids per slurry volume, where }\left(d X / d\left(m_{S} / \mathrm{V}\right)\right)<0 \\
\left(m_{S}(t) / V\right) & =0
\end{aligned}
$$


The caustic leaching step requires a certain temperature at a certain time to dissolve the relatively slowly reacting Boehmite $(\mathrm{AlO}(\mathrm{OH}))$. Gibbsite $\left(\mathrm{Al}(\mathrm{OH})_{3}\right)$ also dissolves essentially instantaneously (compared to Boehmite) at the start of the leaching step. Therefore, the fraction of Gibbsite initially in the solids affects $\mathrm{C}_{\mathrm{AL}(\mathrm{OH})_{4}^{-}, \text {initial }}$ for the caustic leaching of Boehmite.

\subsubsection{Mixing}

Mixing potentially affects $\left(m_{S}(t) / V\right)$, the mass of reacting solids per slurry volume, which affects the chemical conversion and hence the leach factor. If a region is stagnant during the leaching step, the chemical conversion will be unaffected if $\left(m_{S}(0) / V\right)$, the initial value of the solids loading, is the same as for regions that subsequently are mixed because the history $\left(m_{S}(t) / V\right)$ for each is determined by the leaching reaction rate law. However, if solids settle, they will have a higher value of $\left(m_{S}(0) / V\right)$, which will suppress the leaching rate due to excessive accumulation of reaction products in the interstitial liquid around the locally concentrated solids. This results in a lower conversion over the duration of the leaching step, which will lower the leach factor obtained for the vessel. Thus, it is important to prevent settling in order to provide a uniform value of $\left(m_{S}(t) / V\right)$ over the vessel.

\subsubsection{Agitation}

The same hydrodynamic processes that mix the slurry also agitate the fluid around solid particles. As discussed in Appendix A, Section A.2.2.2, this can potentially affect the leaching rate by affecting the concentrations of both reagents and reaction products at the reaction surface compared to the concentrations accumulating or decreasing in the liquid surrounding a particle. As discussed in Appendix A, Section A.2.2.3, an order-of-magnitude analysis of diffusion in surrounding embedded particles is sufficiently rapid, even if the particle is motionless relative to the liquid, to prevent concentration differences between a particle surface and surrounding liquid. Therefore, we do not expect agitation to increase the leach rate by increasing diffusion rates around particles. Note that we have distinguished "agitation" around individual particles from "mixing," which by redistributing solids over the total liquid can affect the local volume ratio of interstitial liquid to solids.

This conclusion can be tested by changing the agitation rate by changing the PJM nozzle velocities. However, any effect observed must be discerned from an effect of increased velocity on settling of particles and its consequence to the concentration in the interstitial liquid of reaction products. That is, because particle agitation and suspension of solids are both facilitated by increasing the PJM nozzle velocity, one must be sure only one effect is dominant before drawing conclusions about it.

\subsubsection{Filtration}

Filtration is precluded during operation near the boiling point due to cavitation in the recirculation pump. Consequently, caustic leaching occurs with neither filtration nor mixing provided by circulation through the filter loop.

\subsubsection{Effect of Time Scale}

The temperature, time at temperature, solids loading in slurries, and concentration of caustic are matched between the PEP and PTF (see Section 7.2.3). Therefore, from the functional dependence shown 
in the prior section, and if the unimportance of agitation is confirmed, the chemical conversion will progress at a rate nominally independent of velocities imposed in vessels. That is, neither the time nor temperature for a specified chemical conversion can be manipulated as a test parameter. Accordingly, the duration and temperature of the caustic mixing step are matched between the PEP and the PTF.

The significance of the PJM operation attributes time (i.e., significance of the PJM cycle time and nozzle velocity) to the time scale is discussed in part in Sections 7.2.2 and 7.3.2, except the time constant for comparison is a characteristic leaching time (implicit in the functional dependence described in Section 7.6.1.2) that is matched between the PEP and PTF.

\subsubsection{Technical Guidance}

The first priority is to establish a representative temperature sequence and establish the same or at least known chemical initial conditions in the PEP as in the PTF. This means matching the temperature at corresponding times, and ideally, matching the composition and solids loading at the start of the heating cycle. Recognizing that the actual chemical initial condition depends on the dewatering and washing steps, whose performance itself must be confirmed, the second priority is to maintain representative mixing during caustic leaching so that resulting local solid/liquid ratios create the same chemical conditions as in the PTF. Then given the same temperature and chemical initial condition, as is apparent from the previous sections, the only adjustable parameters during caustic leaching are the PJM operation attributes. The rationale for adjusting them is to vary the extent of agitation of particles and (potentially) the extent of suspension of settled particles (if any settling occurs).

As noted above, the effect of agitation is expected to be small, but this can be tested by measuring leach factors for different ratios of velocities between the PEP and PTF. Given the probable importance of non-Newtonian behavior, matching the PJM nozzle velocities to match yield Reynolds numbers is advisable. However, because the ensuing mixing per cycle is a transient phenomenon, there is no simple correspondence of the nozzle velocity during the drive phase and the resulting yield Reynolds number, time- and volume-averaged over a PJM cycle and over the vessel. Therefore, there is no clear rationale for one choice of velocity scaling over another. However, for two tests it would be reasonable to compare leach factors when matching the velocities (matching the nominal yield Reynolds number as well as ratio velocity/settling velocity) to those when matching the power/volume. Then leach factors would be determined for velocities in the ratio $(4.5)^{1 / 3}$ or 1.65 - enough to test the effect. Considering the reverse perspective, the power/volume, which scales as $\mathrm{u}^{3} / \mathrm{L}$, would be in the ratio 4.5 between the two tests-a significant ratio.

\subsection{Cooling}

\subsubsection{Controlling Phenomena}

The controlling phenomena are as for heating, without the addition of steam. 


\subsubsection{Effect of Length Scale}

The result of the heat balance reduces approximately to

$$
\frac{T_{C}-T_{a}}{T_{0}-T_{a}}=e^{-\sum_{i}^{M} \frac{A_{i} h_{i}}{m C_{p}} t_{C}}
$$

where the initial temperature $T_{0}$ for cooling is the caustic leaching temperature, and the target temperature $T_{C}$ is that at the end of the cooling step, which is attained at time $t_{C}$, and the heat transfer is summed over the $i$-th of $M$ total heat transfer paths, such as through vessel insulation and active heat exchange. The relation is only approximate in that each cooling path can have a different driving temperature difference, but this difference is determined more by the fluid temperature than the differences in heat transfer paths, and the expression suffices to illustrate the effect of length scale. The cooling time is

$$
t_{C}=\sum_{i}^{M} \frac{m C_{p}}{A_{i} h_{i}} \ln \frac{T_{0}-T_{a}}{T_{c}-T_{a}}
$$

which is proportional to the ratio of mass being cooled to area of heat transfer, which is proportional to the length scale. Hence, for given starting, ending, and "driving" temperatures for cooling for the actual heat transfer paths, the cooling time is roughly proportional to the length scale.

\subsubsection{Effect of Time Scale}

The effective environmental heat transfer coefficient, say, $h_{l}$ in the above expression, is affected by the heat transfer coefficient on the interior of the tank walls, which is affected by the fluid motion. However, much of the total heat transfer resistance will be on the exterior, where only radiation and natural convection act to remove heat. Therefore, the effect of fluid motion on the effective $h_{l}$ will be small. Therefore, any changes in fluid motion attempted based on time-similitude will have little effect on the cooling time.

Probably the greatest effect of time scale will be due to the imposition of active heat transfer by circulation through heat exchange equipment if the time scale cannot be controlled adequately by adjusting thermal insulation or other attributes determining the environmental heat transfer coefficient. In that case, the time scale is controlled directly and independently of the length scale. Of course, the active control of such heat transfer is calculated based in part on the length scale because it determines the mass and hence the thermal inertia of the slurry being cooled.

\subsubsection{Technical Guidance}

For the reasons stated in Section 7.5.3, the leach factors observed will be difficult to interpret unless the temperature history is the same between the PEP and PTF. We can achieve a prototypic cool-down time and $\mathrm{T}$ vs t curve by non-prototypic control of the water jacket in UFP-2. This could include quenching the UFP-1 caustic leach over a prototypic duration by rapidly cooling to, say, $85^{\circ} \mathrm{C}$ with the external chillers, then cooling further by prototypic heat transfer. 


\subsection{Oxidative Leaching}

Oxidative leaching consists of reacting slurry solids with sodium permanganate at filtration temperatures to convert insoluble chromium compounds into to soluble sodium chromate.

\subsubsection{Controlling Phenomena}

Similar to Boehmite dissolution by caustic, the oxidative leaching reaction occurs at particle-liquid interfaces. However, even at modest temperatures, the reaction rate is much faster than for Boehmite, and a reaction rate law has not been determined and is not expected to be needed. Instead, the chemical conversion is expected to be complete so long as the reactants are mixed well with the reacting solids. Therefore, mixing of the reagent with the slurry is the pertinent controlling phenomenon. This is discussed in Section 8.4.

\subsubsection{Effect of Length Scale}

Chemical conversion by oxidative leaching does not depend on the length scale.

\subsubsection{Effect of Time Scale}

The rate of oxidative leaching is great enough that we do not characterize it by a reaction rate law, and there is no obvious characteristic time scale for it. Nominally the effect of the time scale is only through the effect of velocity on mixing analogous to the discussion in Sections 7.6.1.3 and 7.6.1.4. However, there are additional opportunities during oxidative leaching to investigate the effect of mixing and agitation because a) the recirculation loop can be operated at the modest temperatures of this step, b) in the absence of simultaneous filtration the velocities in the filter tubes is not specified, and c) the filter loop pumps can be operated over a range of flow rates.

\subsubsection{Technical Guidance}

As for caustic leaching, the first priority is to match the temperature sequence and the chemical initial conditions between the PEP and the PTF. Secondarily, it is desirable to make the mixing conditions representative. In the case of oxidative leaching, the temperature will be low enough to allow mixing by circulation through the filter loop in addition to by the PJMs. Essentially the same arguments prevail as presented in Section 7.6.3.

\subsection{Operating Philosophy}

The PEP is designed to simulate the performance of the existing design of the PTF rather than the PTF design being determined from exploratory testing using the PEP. Consequently, the PEP should be operated to represent the actual behavior of the PTF, whatever it may be.

For the PEP to be a credible predictor of the performance of corresponding sub-systems in the PTF requires not only that the PEP and PTF be physically similar and operated to invoke similar physical and chemical behavior, but also that the PEP be operated based on the same set of process control information as the PTF. Hence, the PEP should be operated using the same limitations on information as for the PTF; 
that is, not all of the available information acquired to meet the purposes of the PEP should be made available to the operators. The notable differences between the PTF and PEP in the information available for process control include:

- extensive sampling and analysis of samples for the PEP versus very few for the PTF

- measurements of temperature over PEP tanks versus a single temperature measurement in PTF tanks.

Thus, to meet the above objective, the PEP must be operated using a small fraction of the available information. That is, there must be a credible separation between information used to operate the PEP and information used to relate the PEP performance to that of the PTF. 



\subsection{Conclusions}

\subsection{Rationale for the PEP Scale of 4.5}

The desire to test full-scale filter assemblies leads to a scale factor derived from the ratio of the filter lengths in the single assembly to the WTP number of assemblies, resulting in a scale factor of 4.5 . Furthermore, the scale factor of 4.5 is consistent with the desire for a value of less than 5 to replicate fullscale mixing as closely as reasonably practical and to minimize uncertainty in interpreting mixing data.

\subsection{Technical Basis for the Approach in Scaling PEP Performance Data to Predict WTP Performance}

There are two principal means of predicting the performance of the PTF from operation of the PEP. First, we can apply principles of similitude to interpret PEP data as virtual PTF data as though we were operating the PTF. Unfortunately, no set of adjustable parameters establishes full similitude between the PEP and the PTF, and therefore, not all predictions of PTF performance can be based on similitude between the PEP and PTF. Instead, we must optimize what can be deduced from similitude and dimensional analysis and apply it in terms of the simplest relationships between PTF performance and PEP data.

Second, we can predict the performance of both the PTF and PEP from their design basis-i.e., from understanding the process chemistry and process operations on which the design of the UFP system is based. To the extent that the PEP is designed so its performance is controlled by the same phenomena that will control the performance of the PTF, the design basis for the PEP and PTF are the same, in which case confirming the PEP design basis also confirms the PTF design basis.

\subsection{Use of PEP Performance Data to Predict WTP Performance}

Observations in the PEP can be compared to predictions based on ideal mixing to identify and quantify departures from ideal mixing, and from this, any needed corrections to predictions of performance in the WTP can be developed based on ideal mixing combined with mass and heat balances and laboratory data.

Departures from ideal behavior in the PEP or the WTP will probably be small because the systems are conservatively designed to be well-mixed. However, even if true, this may not be obvious because any test of the data against this assumption inevitably involves both actual departures from ideal behavior and errors in measuring variables that describe departures from ideal behavior. Thus, these contributions to the error must be separated to evaluate the correctness of the presumed model. Therefore, statistical methods should be applied that provide estimates of the true values of measured variables while simultaneously imposing constraints representing the presumed ideal behavior.

\subsection{Scaling Issues of PEP Design, Their Potential Impacts, and Mitigation Approaches}

Because only two choices are available to scale the design and operation of the PEP (length and time through velocity), very few physical phenomena in the WTP can be directly represented by observations 
in the PEP by enforcing similitude. The length and time scales for the PEP have been chosen to make observations in the PEP of the degradation of filtration performance and the effects of any non-Newtonian behavior on mixing directly representative of corresponding behavior in the WTP. Phenomena controlled by competition among forces different from those controlling the above phenomena must be interpreted and used to predict behavior in the WTP based on an understanding of the phenomena. Technical guidance based on phenomena particularly important to each of the process steps is provided in the "technical guidance" sub-sections in Section 7.

Simple conceptual models of non-ideal mixing to be used as a basis for correcting, if necessary, ideal mass balance models implicit in the PTF and PEP design bases are postulated in Appendix A, Section A.3. However, the very means of testing for ideal mixing or correcting ideal mass balances to address non-ideal mixing are themselves subject to interpretation of the data. That is, inevitably we will learn from the data how best to interpret the data.

The PJMs utilize transient submerged jets to agitate fluid in a tank. We have used information about steady jets to infer the effect of PJM parameters on mixing using principles of similitude and dimensional analysis. These inferences are tenuous without applicable data on transient jets. Thus, the recommendations in this report are subject to revision if more experience with transient systems is acquired, such as through tests conducted as part of the M3 task. 


\section{Appendix A}

Process Details and Physico-Chemical Phenomena 



\section{Appendix A}

\section{Process Details and Physico-Chemical Phenomena}

\section{A.1 Similitude and Dimensional Analysis}

Diverse aspects of similitude and dimensional analysis enable - or complicate - treating operation of the PEP as virtual operation of the PTF and predicting the performance of the PTF as that in the PEP. These include:

- Similitude

- application of first principles: similitude in conservation laws

- boundary, kinematic, and dynamic similitude

- practical constraints in applying principles of similitude

- Dimensional analysis:

- application of first principles: dimensional analysis of conservation laws

- dimensional analysis of empirical information

- time scale: characteristic process times.

These are described in the following sections.

\section{A.1.1 Similitude in Conservation Laws}

The hydrodynamic, thermal, and chemical behavior in a system - i.e., the distribution and transport of momentum, heat, and multi-component mass - is governed by the conservation of momentum, energy, and mass. They have a common differential (meaning describing distributions over space and time) form, as follows:

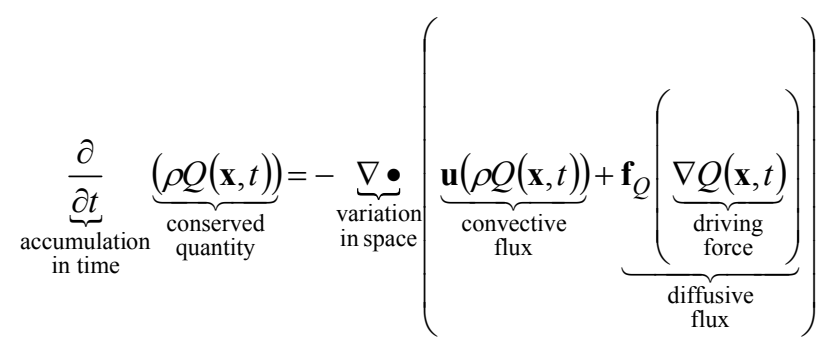

where $Q(\mathbf{x}, t)$ is a conserved scalar or vector quantity per mass generally varying over both space $\mathbf{x}$ and time $t, \mathbf{u}$ is the velocity vector of the fluid, $\mathbf{f}_{Q}$ is the diffusive flux vector or tensor of $Q$ relative to the fluid velocity, which is a function of the gradient in $Q$. The meaning of $\mathrm{Q}$ and $\mathbf{f}_{\mathrm{Q}}$ is shown in Table A.1, which identifies the generalized stresses driving the diffusive transport of the conserved quantities. The diffusive transport of momentum is actually a mechanical stress, which needs to be distinguished from the concept of the generalize stress driving the diffusive transport. Thus, for the case of momentum, the generalized stress is the momentum gradient (velocity gradient) and the resulting diffusive momentum flux is momentum transport, which is a mechanical stress. 
Table A.1. Generalized Stresses Driving Diffusive Transport

\begin{tabular}{||l|c|c|c|c||}
\hline \hline Conservation Law & $\begin{array}{c}\text { Conserved } \\
\text { Quantity } \\
\text { per Volume Q }\end{array}$ & $\begin{array}{c}\text { Convective } \\
\text { Flux } \\
\mathbf{u}(\mathbf{Q})\end{array}$ & $\begin{array}{c}\text { Diffusive } \\
\text { Flux } \\
\mathbf{f}_{\mathrm{Q}}\end{array}$ & $\begin{array}{c}\text { Diffusive } \\
\text { Stress } \\
\nabla Q(\mathbf{x}, t)\end{array}$ \\
\hline \hline Conservation of momentum & $\rho \mathbf{u}$ & $\mathbf{u}(\rho \mathbf{u})$ & $\begin{array}{c}\text { Mechanical Stress } \\
\boldsymbol{\sigma}\end{array}$ & $\nabla(\rho \mathbf{u})$ \\
\hline Conservation of energy & $\rho C_{p}\left(T-T_{0}\right)$ & $\mathbf{u}\left(\rho C_{p}\left(T-T_{0}\right)\right)$ & $\begin{array}{c}\text { Heat conduction } \\
\mathbf{q}\end{array}$ & $\nabla\left(\rho C_{p} T\right)$ \\
\hline Conservation of mass (of $i)$ & $\rho_{i}$ & $\mathbf{u}\left(\rho_{i}\right)$ & $\begin{array}{c}\text { Diffusion of } i \\
\mathbf{j}_{i}\end{array}$ & $\nabla\left(\rho_{i}\right)$ \\
\hline
\end{tabular}

One can consider the gradients in $Q$ as generalized stresses driving the diffusive flux. Hence, the conservation laws relate the distribution of $Q$ over space and time to the velocity field $\mathbf{u}$, which is also distributed over space and time, and (through the diffusive flux) the distribution of generalized stress over space and time. Thus, the conservation law is

$$
\frac{\partial Q}{\partial t}=-\nabla \bullet\left(\mathbf{u} Q+\mathbf{f}_{Q}(\nabla Q)\right)
$$

which, conceptually (and mathematically, when tractable) integrates implicitly to

$$
\mathbf{f}_{Q}=\mathbf{f}_{Q}\left(\nabla Q ; \Gamma_{Q}\right)
$$

if the velocity field $\mathbf{u}$ is known (determined from the conservation of momentum), where $\Gamma_{Q}$ denotes the boundary on which the boundary conditions for $Q$ or $\mathbf{f}_{Q}$ are specified. That is, through the conservation laws, the fluxes implicitly depend on the boundary conditions and the stresses $\nabla Q$. Clearly, the velocity field $\mathbf{u}$ plays a special role, being the basis for convective transport of all quantities, including momentum. Hence the conservation of momentum, which determines the velocity field, is of special importance and is emphasized in Section A.1.4.2.

The above expression shows that changes in conserved quantities are described, given the velocity field, in terms of diffusive fluxes that depend on the spatial distribution of the conserved quantities. Then implicitly, if one establishes geometrically similar patterns of gradients in conserve quantities acting within the geometrically similar boundaries and boundary conditions, one establishes geometrically similar patterns of the resulting fluxes. Integrating the fluxes over the boundaries describes the transport of mass (or volume) and chemical components throughout the UPF, and hence ultimately describes the principal performance measures: filter flux (volumetric flow rate), wash factor (mass flows of chemical components as solid and dissolved species), and leach factors (mass flows of chemical components as solid and dissolved species) because the distribution of components between solid and dissolved species depends ultimately on concentrations and temperatures over time, which are controlled by the conservation laws.

\section{A.1.2 Three Components of Similitude}

Implicit from the above is that the geometric pattern of the total (convective and diffusive) flux depends on the spatial variation - i.e., depends on the geometric pattern — of the stresses driving the 
diffusive flux, which are determined by gradients in the conserved quantity (momentum, energy, or mass) - i.e., depend on the geometric pattern of the generalized stresses. Thus, geometric similarity of the patterns of total flux is established by geometric similarity of the pattern of generalized stresses driving the total flux.

Hence, we can consider three forms of similitude:

- boundary similitude: geometric similitude (the same shape) of boundaries and the same distribution of boundary conditions over boundaries, depicted in Figure A.1.

- kinematic similitude: geometric similitude (the same shape) of the distribution of generalized fluxes, existing both in the interior of a system and on its boundaries

- dynamic similitude: geometric similitude (the same shape) of the distribution of generalized stresses, acting both in the interior of a system and on its boundaries
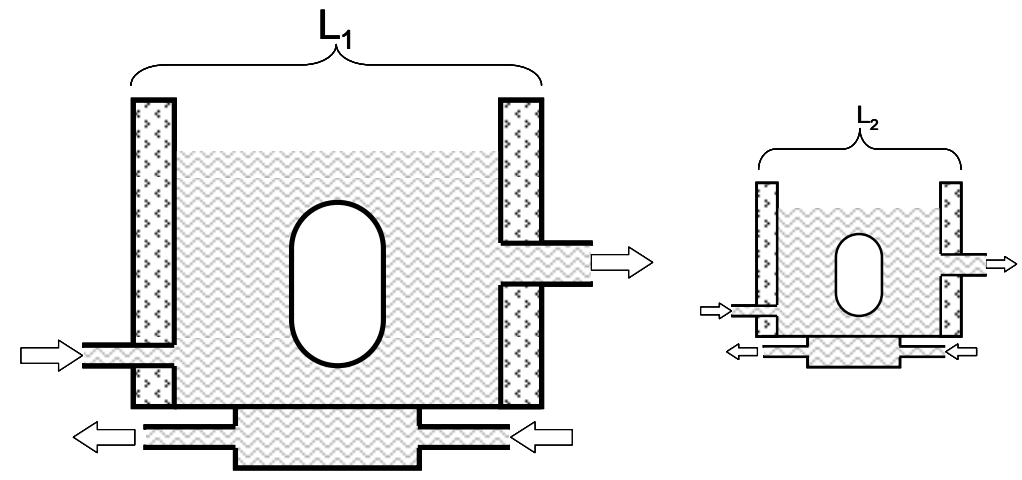

Figure A.1. Depiction of Boundary Similitude between Hypothetical Pieces of Equipment

Figure A.2 illustrates establishing similitude between systems characterized by different lengths (sizes) and times (velocities). At the top left, the initial instantaneous pattern of associated stresses and fluxes is shown at an early time, and at the top right, at a later time. Note the evolution of the pattern with time. The length scale at the top is $L_{l}$ and the difference in time to attain a certain change in the pattern is $\Delta t_{A}$. At the bottom left, again an initial instantaneous pattern is shown, and at the bottom right the evolution of the pattern at length scale $\mathrm{L}_{2}$ after time difference $\Delta \mathrm{t}_{\mathrm{B}}$ is shown where it has evolved to the same extent as for time difference $\Delta \mathrm{t}_{1}$ at length scale $\mathrm{L}_{1}$. The shape of the patterns of stresses and fluxes at the start and the shapes after the time difference are the same, signifying similitude between the two systems. As depicted, the same pattern is obtained at short times at a large size and at long times at a small size. This implies a certain time-size relationship for whatever stresses and fluxes are represented. The pattern depicted was chosen arbitrarily for the figure. The actual relationship depends on the quantities being conserved and the physical situation. The point of Figure A.2 is that the shape of the patterns is the same at corresponding times; that is, there is geometric similarity of the patterns of generalize fluxes (generalized kinematic similitude) and generalized stresses (generalized dynamic similitude). 

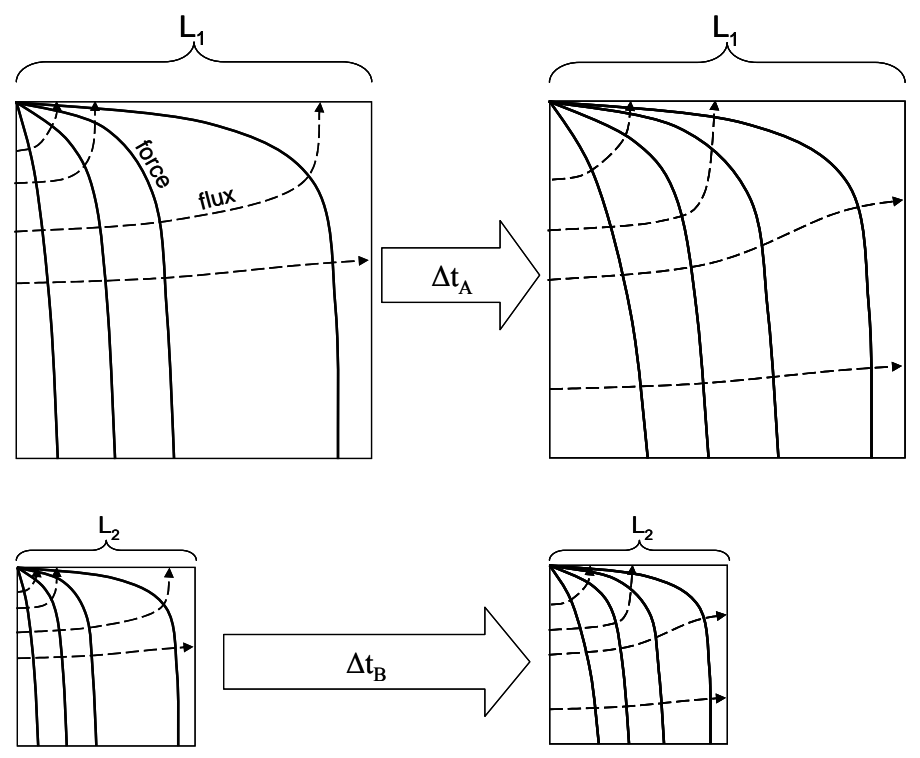

Figure A.2. Depiction of Generalized Kinematic and Dynamic Similitude Between Differing Length and Time Scales (See text for explanation)

The performance of the PTF is defined in terms of fluxes: multicomponent mass transferred (the basis of wash and leach factors) and mass transferred (separation of liquid from solids by filtration). This requires kinematic similitude (see Section 2.2) between the PEP and PTF so that the pattern of fluxes observed in the systems will have the same shape and hence the patterns observed in one system represent those that would occur in the other. This is the essential objective of establishing similitude (boundary, kinematic, and dynamic) between systems: so that behavior observed in one system represents (is geometrically similar to and proportionate in time to) behavior occurring in the other.

Kinematic similitude is established by boundary similitude and dynamic similitude. In designing and operating the PEP, one can control the shape of the boundaries and the generalized stresses but not directly the fluxes except through boundary conditions. The equipment in PEP is designed to establish boundary similitude.

\section{A.1.3 Practical Constraints on Similitude}

The actual phenomena controlling the performance of the UFP in the PTF are physically and chemically complex, involving chemically reacting slurries of chemically diverse particles spanning particle size distributions of several orders of magnitude. Hydrodynamic interactions among particles can produce non-Newtonian rheological behavior. The fluid motion of greatest importance is turbulent, prominently transient, and prominently inhomogeneous.

While it is important to understand and optimally apply principles of similitude, it is equally important to understand and accept that

- no set of adjustable parameters establishes full similitude between the UFP in the PEP and the PTF, and therefore

- not all predictions of PTF performance can be based on similitude between the PEP and PTF. 
Instead, we must optimize what can be deduced from similitude and dimensional analysis and (mainly) apply it in terms of the simplest relationships between PTF performance and PEP data. Furthermore, the only practical available parameter to adjust to establish any aspect of similitude is the characteristic velocity, or equivalently the hydrodynamic characteristic time.

Aside from being limited to affecting similitude only by adjusting the velocity, the general approach to similitude must be empirical because the system is far too complicated to extract credible conditions for similitude only from first principles (i.e., dimensional analysis of the conservation laws, described in Section A.1.4). Instead, we must base key decisions on dimensional analysis applied to experiments relevant to the behavior in the PTF we want to simulate in the PEP. An example of this is the correlation of settling experiments described in Section A.3.2.2.

On the other hand, approximate mechanistic analysis can be combined with dimensional analysis to provide useful conditions of similitude. An example of this is the analysis of the simultaneous effect of turbulent dispersion and settling developed in Section A.1.4.4.

\section{A.1.3.1 Limitations on Number of Adjustable Parameters}

One can control boundary similitude through the design of the equipment in which the hydrodynamic, thermal, and chemical phenomena occur. However, dynamic similitude is a matter of generalize stresses; only a few attributes of a system affecting generalized stresses can be adjusted while maintaining boundary similitude, which includes geometric similarity of the conditions at the boundaries.

Regarding thermal and chemical generalized forces, one can choose different characteristic temperature differences and characteristic concentration differences to impose dynamic similitude. However, the interrelationships of temperature and concentration in a complex physical and chemical system are too complex on which to ensure similitude. The only practical choice is to operate the systems at the same temperature (at corresponding times) and same concentrations (e.g., solids volume fractions in the feed to the process).

Regarding hydrodynamic stresses, where the boundary is solid and rigid, the pressure and velocity are controlled by the conservation of mass and momentum. Where the boundary is a flow inlet, one can control the velocity at that point. If there are multiple inlets, the ratios of velocities at the inlets must be the same between systems to establish boundary similarity. This leaves only a single velocity as a parameter available to adjust to establish dynamic similitude. Where the boundary is a free surface, one can control the pressure (e.g., atmospheric), in which case the local velocity and shape of the surface are controlled by the conservation of mass and momentum. In any event, variation of the nature of the boundary (solid/rigid, inlet/outlet, or free) over the entire boundary of one system must be geometrically similar to the variation in another system, to establish boundary similitude.

\section{A.1.3.2 Reduction to Characteristic Length and Velocity}

For the applicable case of imposing atmospheric pressure at free boundaries (i.e., top liquid surfaces in tanks), one has available only two adjustable parameters defined by the equipment and its operation:

- the characteristic size, and

- the characteristic velocity. 
Any other choice pertinent to hydro-dynamic similarity must involve the material properties, which mainly depend on the choice of material and to some extent the temperature and chemical composition. Then if one processes the same material in two systems, one has available only choices in the size and velocity.

The PEP is designed and being constructed; hence, its size is fixed. Given the same temperature and concentrations, one has only the choice of velocity in the PEP to affect dynamic similitude between the PEP and the PTF. Obviously, one cannot control all of the stress ratios listed above by changing the characteristic velocity. Consequently,

- we must decide which stress ratio is most important, and choose the characteristic velocity on that basis, and

- we must understand the consequences — or lack thereof — of not matching other ratios of generalize stresses.

\section{A.1.4 Dimensional Analysis of Conservation Laws}

The hydrodynamic, thermal, and chemical behavior in a system-i.e., the distribution and transport of momentum, heat, and multi-component mass - is governed by the conservation of momentum, energy, and mass. One can think of the gradients in conserved quantities as generalized stresses driving the diffusive fluxes that add to the convective contribution to the transport of the conserved quantities, which depends on the velocity field, which is determined by the conservation of momentum. Hence, the conservation laws combined relate all the fluxes to the distribution of all conserved quantities over space and time.

Implicit from the discussion in Section A.1.1 is that the geometric pattern of the total (convective and diffusive) flux depends on the spatial variation - i.e., depends on the geometric pattern - of the stresses driving the diffusive flux, which are determined by gradients in the conserved quantity (momentum, energy, or mass) - i.e., depend on the geometric pattern of the generalized stresses. Thus, geometric similarity of the patterns of total flux is established by geometric similarity of the pattern of generalized stresses driving the total flux.

Conservation Laws relate the accumulation of conserved quantities to convective fluxes and diffusive fluxes. The convective fluxes involve the velocity, which is proportional to the flux of momentum. Hence, the conservation of momentum is central to all conservation laws and the dimensional analysis of them.

\section{A.1.4.1 Dimensional Analysis of Conservation of Momentum}

The flow of incompressible fluids is described by the conservation of momentum, which is

$$
\underbrace{\frac{\partial}{\partial t}(\rho \mathbf{u})}_{\begin{array}{c}
\text { accumulation } \\
\text { of momentum }
\end{array}}=-\nabla \bullet \underbrace{\mathbf{u}(\rho \mathbf{u})}_{\begin{array}{c}
\text { convection } \\
\text { of momentum }
\end{array}}-\nabla \bullet \underbrace{\boldsymbol{\tau}}_{\begin{array}{c}
\text { shear forces } \\
\text { acting on } \\
\text { the fluic }
\end{array}}-\nabla \underbrace{p}_{\begin{array}{c}
\text { normal } \\
\text { forces } \\
\text { action on } \\
\text { the fluid }
\end{array}}+\underbrace{\rho \mathbf{g}}_{\begin{array}{c}
\text { body forces } \\
\text { acting on } \\
\text { the fluid }
\end{array}}
$$


where $t$ is time, $\rho$ is the fluid density, $\mathbf{u}$ is the velocity vector (throughout, bold font denotes a vector or tensor), $\boldsymbol{\tau}$ is the shear stress tensor induced by fluid flow, $\nabla \bullet$ is the divergence operator, $\nabla$ is he gradient operator, $p$ is the pressure, and $\mathbf{g}$ is the acceleration vector of gravity. When the fluid is static we have

$$
0=-\nabla p+\rho \mathbf{g}=-\nabla(p+\rho g z)=-\nabla p_{z}
$$

where $p_{z}$ is the "dynamic pressure"- the pressure relative to the hydrostatic pressure. We make this equation dimensionless in time and spatial dimensions by defining $\widetilde{t}=t / t_{0}, \widetilde{\mathbf{u}}=\mathbf{u} / \mathbf{u}_{0}$ and $\widetilde{\nabla}=L_{0} \nabla$ where $\mathrm{t}_{0}, \mathrm{v}_{0}$, and $\mathrm{L}_{0}$ are a characteristic time, velocity, and length for the system in which the fluid flows. Further, for an incompressible fluid the density is constant. Substituting,

$$
\frac{L_{0}}{\mathrm{u}_{0} t_{0}} \frac{\partial \widetilde{\mathbf{u}}}{\partial \widetilde{t}}=-\tilde{\nabla} \bullet(\widetilde{\mathbf{u}} \widetilde{\mathbf{u}})-\widetilde{\nabla} \bullet\left(\frac{\boldsymbol{\tau}}{\rho \mathrm{u}_{0}^{2}}\right)-\nabla\left(\frac{p_{z}}{\rho \mathrm{u}_{0}^{2}}\right)
$$

where $\Delta p_{0}=\rho \mathrm{u}_{0}^{2}$ is the characteristic inertial (stagnation) pressure. We cannot independently choose the characteristic length, time, and velocity — one is determined by the other two. Experimentally, we can most directly control the characteristic length and velocity in the system. Hence, choose $t_{0}=L_{0} / \mathrm{u}_{0}$ as the characteristic time. Then, using analogous notation for dimensionless forms of the stress tensor and dynamic pressure,

$$
\frac{\partial \widetilde{\mathbf{u}}}{\partial \widetilde{t}}=-\widetilde{\nabla} \cdot(\widetilde{\mathbf{u}} \widetilde{\mathbf{u}})-\widetilde{\nabla} \bullet \widetilde{\boldsymbol{\tau}}-\nabla \widetilde{p}_{z}
$$

Finally, we can use the identity

$$
\widetilde{\nabla} \tilde{p}_{z}=\widetilde{\nabla} \bullet\left(\boldsymbol{\delta} \tilde{p}_{z}\right)
$$

where $\boldsymbol{\delta}$ is the unit tensor (components are the Kronecker delta function) and define the total stress as

$$
\tilde{\boldsymbol{\sigma}} \equiv \tilde{\boldsymbol{\tau}}+\boldsymbol{\delta} \tilde{p}_{z}
$$

Then we can write the conservation of momentum as

$$
\frac{\partial \widetilde{\mathbf{u}}}{\partial \widetilde{t}}=-\widetilde{\nabla} \cdot(\widetilde{\mathbf{u}} \widetilde{\mathbf{u}})-\widetilde{\nabla} \cdot \widetilde{\boldsymbol{\sigma}}
$$

The left side is the rate of change of the dimensionless momentum per mass; the right side is, first, the divergence of the dimensionless convective flux of momentum per mass, and, second, the divergence of the dimensionless diffusive flux (flux relative to fluid motion) of momentum per mass driven by gradients in the momentum per mass. The diffusive flux of momentum is the total stress and vice versa; they are interchangeable concepts. 


\section{A.1.4.2 Similitude Based on Dimensionless Conservation of Momentum}

It follows that for systems with geometrically similar boundaries, the same function $\widetilde{\mathbf{u}}(\tilde{t}, \widetilde{\mathbf{x}})$ will be observed, where $\widetilde{\mathbf{x}}$ is the position vector, if the same function $\boldsymbol{\tau}=f(\nabla \mathbf{u})$ describes the stress-strain relation for the fluid. For incompressible Newtonian fluids, this is

$$
\tau=-\mu \Delta
$$

where

$\Delta=\nabla \mathbf{u}+(\nabla \mathbf{u})^{t}$ is the rate of deformation tensor,

$\nabla \mathbf{u}$ is the velocity gradient tensor, and

$(\nabla \mathbf{u})^{t}$ is the transpose of the velocity gradient tensor.

Then for a Newtonian fluid,

$$
\nabla \bullet \boldsymbol{\tau}=-\nabla \mu \bullet \nabla \mathbf{u}-\nabla \mu \bullet(\nabla \mathbf{u})^{\mathrm{t}}-\mu \nabla \bullet \nabla \mathbf{u}-\mu \nabla(\nabla \bullet \mathbf{u})
$$

Commonly, the spatial variation in viscosity is not significant (if the viscosity does vary significantly due to concentration effects, an additional parameter is introduced; if the viscosity varies significantly due to the velocity gradient, the fluid is not Newtonian), and for incompressible fluids the last term vanishes. Then

$$
-\widetilde{\nabla} \bullet \frac{\boldsymbol{\tau}}{\rho u_{0}^{2}}=\frac{\mu}{\rho u_{0}^{2}} \widetilde{\nabla} \bullet \nabla\left(u_{0} \widetilde{\mathbf{u}}\right) \equiv \frac{\mu u_{0}}{\rho u_{0}^{2} L_{0}} \widetilde{\nabla}^{2} \mathbf{u}=\frac{1}{\operatorname{Re}} \widetilde{\nabla}^{2} \widetilde{\mathbf{u}}
$$

where $R e=\rho u_{0} L_{0} / \mu$ is the Reynolds number. The dimensionless conservation of momentum becomes

$$
\frac{\partial \widetilde{\mathbf{u}}}{\partial \widetilde{t}}+\widetilde{\nabla} \bullet(\widetilde{\mathbf{u}} \widetilde{\mathbf{u}})=\frac{\partial \widetilde{\mathbf{u}}}{\partial \widetilde{t}}+\widetilde{\mathbf{u}} \bullet \widetilde{\nabla} \widetilde{\mathbf{u}}=-\frac{1}{\operatorname{Re}} \widetilde{\nabla}^{2} \widetilde{\mathbf{u}}-\nabla \widetilde{p}_{z}
$$

and hence we expect $\widetilde{\mathbf{u}}=\mathbf{g}(\widetilde{\mathbf{x}}, \widetilde{t}, \mathrm{Re})$ where $\mathbf{g}$ is a dimensionless vector function to be determined-e.g., by correlating data-from experimental results.

This is always observed for an incompressible, pure component, Newtonian fluid, although the actual dependence on the Reynolds number can be profound or negligible, depending on the situation.

For the case of turbulent flow, the equation is still valid but the solution is unstable, reflecting the instability of the flow itself. The stability of the flow is characterized by the Reynolds number, and for increasing $R e$ the flow in increasingly unstable and ultimately increasingly turbulent, yet for very turbulent flows the structure tends to become independent of Reynolds number.

For turbulent flows, time-averaged measurements of the velocity field can be compared with predictions from the time-averaged conservation of momentum, which must be described in terms of a time-averaged velocity and fluctuations about it. For Newtonian fluids the stress tensor $\tilde{\boldsymbol{\tau}}(\nabla \widetilde{\mathbf{u}})$ is linear in the velocity gradient tensor $\nabla \widetilde{\mathbf{u}}$ and can be reduced to a single material property" viscosity. However, 
time averaging of fluctuating components of the convective transport of momentum introduces a shear stress-like function non-linear in the gradient of the time-averaged velocity that is proportional to the density and not a function of the viscosity. In strongly turbulent flow, this inertial stress term dominates the viscous stress term. For such flows, the viscosity is not important.

For yield stress (Bingham plastic) fluids, the stress-strain relationship is not Newtonian (i.e., it is a "non-Newtonian" fluid), but instead

$$
\tau=-\mu_{0} \Delta-\tau_{0}(\Delta /|\Delta|)
$$

where $\tau_{0}$ is the yield stress and $|\boldsymbol{\Delta}|$, a scalar, is the magnitude of the rate of deformation tensor $\Delta$; i.e., $|\Delta|=\sqrt{\Delta: \Delta}$.

\section{A.1.4.3 Generalization to All Conservation Laws}

We obtain analogous results from the conservation of energy and the conservation of multicomponent mass. From the conservation of energy,

$$
\frac{\partial \widetilde{U}}{\partial \widetilde{t}}=-\widetilde{\nabla} \bullet(\tilde{\mathbf{u}} \widetilde{U})-\widetilde{\nabla} \bullet \widetilde{\mathbf{q}}
$$

where $\widetilde{U}$ is the dimensionless internal energy per mass and $\widetilde{\mathbf{q}}$ is the dimensionless heat flux. The left side is the time-scaled rate of change of the dimensionless internal energy per mass; the right side is, first, the divergence of the dimensionless convective flux of internal energy per mass, and, second, the divergence of the dimensionless diffusive flux (flux relative to fluid motion) of internal energy per mass per mass driven by gradients in the internal energy per mass.

From the conservation of multicomponent mass,

$$
\frac{\partial w_{i}}{\partial \widetilde{t}}=-\tilde{\nabla} \bullet\left(\widetilde{\mathbf{u}} w_{i}\right)-\widetilde{\nabla} \bullet \widetilde{\mathbf{j}}
$$

where $w_{i}$ is the mass fraction of component $i$ and $\tilde{\mathbf{j}}_{i}$ is the dimensionless mass flux of component $i$. The left side is the time-scaled rate of change of the mass fraction of $i$; the right side is, first, the divergence of the dimensionless convective flux of component $i$, and, second, the divergence of the dimensionless diffusive flux (flux relative to fluid motion) of component $i$ driven by gradients in the mass fraction of component $i$.

Hence, the results of the conservation laws are analogous in that each relates the time-scaled change of a quantity per mass to the divergence of the convective flux and the divergence of the diffusive flux of that quantity, of the form

$$
\frac{\partial \widetilde{Q}}{\partial \widetilde{t}}=-\widetilde{\nabla} \bullet(\widetilde{\mathbf{u}} \widetilde{Q})-\widetilde{\nabla} \bullet \widetilde{\mathbf{f}}_{Q}
$$


where $\widetilde{Q}$ is the quantity per volume (made dimensionless by normalizing to some characteristic value) and $\widetilde{\mathbf{f}}_{Q}$ is its dimensionless diffusive flux (flux relative to the convective flux, $\widetilde{\mathbf{u}} \widetilde{Q}$ ), which depends on the dimensionless gradient $\widetilde{\nabla} \widetilde{Q}$. Thus, we can consider $\widetilde{Q}$ as both the conserved quantity (time derivative) and the stress (spatial derivative) driving the diffusive flux, $\widetilde{\mathbf{f}}_{Q}$. Put concisely, these are interrelated such that the generalize fluxes are functions of the generalized stresses for a given boundary configuration.

\section{A.1.4.4 Example: Application to Turbulent Dispersion of Suspended Solids by Submerged Jets}

A submerged, fully turbulent jet penetrating (nominally) a semi-infinite domain is defined by its local velocity and diameter as a function of the distance along the jet (distance into the semi-infinite domain), which ultimately must be determined by the jet's initial velocity and diameter and the density of the fluid (the relative unimportance of viscosity defining "fully-turbulent" flow). In the ideal, instructive case of a fluid without a yield stress and where the jet is not transversely constrained by static boundaries, the jet does not encounter axial forces in a semi-infinite fluid, and hence its momentum flow, $J$, is conserved. Then $J$ remains at its initial value, which is

$$
J_{0}=A_{0} \rho u_{0}^{2}
$$

where $A_{0}$ is the area of the inlet nozzle and $u_{0}$ is the inlet velocity. The local area must depend on the local attributes of the jet: the velocity, jet length, momentum flow, and fluid density. Dimensional analysis shows that the only possible dimensionally consistent dependence is that the area is proportional to the square of the jet length, which for constant momentum flow requires that the local velocity vary inversely with the jet length, and also the diameter is proportional to the length $z$. Thus,

$$
u=u_{0}\left(z_{0} / z\right)
$$

where $\mathrm{z}$ is the length of the jet to the point of observation, and $\mathrm{z}_{0}$ is the effective length at which the jet becomes self-similar, denoting that the shape of the transverse velocity profile becomes independent of $z$.

The rate of turbulent mixing in the jet can be estimated from dimensional analysis and the Corrsin time scale ${ }^{1}$; the proportionality is

$$
t_{m i x}^{3} \sim D(z)^{2} / \varepsilon
$$

where $t_{m i x}$ is the characteristic time scale for turbulent mixing, the local characteristic jet dimension is $D(z)$, the jet diameter at distance $z$ along the jet, and $\varepsilon$ (units of $L^{2} / t^{3}$ ) is the rate of kinetic energy dissipation per unit mass in the jet, which is $\varepsilon=-(\tau: \Delta) / \rho)$. For Newtonian fluids, this is $\varepsilon=v(\Delta: \Delta)$ where $v=\mu / \rho$ is the kinematic viscosity. For a circular jet, then

$$
\varepsilon=v(\Delta: \Delta)=v\left(\frac{\partial u}{\partial r}\right)^{2}=\frac{v u_{0}^{2}}{L_{0}^{2}}\left(\frac{\partial \widetilde{u}}{\partial \widetilde{r}}\right)^{2}=\frac{v}{u_{0} L_{0}} \frac{u_{0}^{3}}{L_{0}}\left(\frac{\partial \widetilde{u}}{\partial \widetilde{r}}\right)^{2}=\frac{u_{0}^{3}}{L_{0}}\left[\frac{1}{\operatorname{Re}}\left(\frac{\partial \widetilde{u}}{\partial \widetilde{r}}\right)^{2}\right]
$$

\footnotetext{
${ }^{1}$ Handbook of Industrial Mixing. 2004. Edward Paul, Victor Atiemo-Obeng, and Suzanne Kresta (editors), WileyInterscience, Hoboken, New Jersey (See Section 9-5.2, "Jet Mixer Design Method").
} 
As is discussed above, we have $\widetilde{u}=\widetilde{u}(\widetilde{r}, t, \mathrm{Re})$. Therefore, for a steady turbulent flow, the term in brackets is a function of the Reynolds number. Also, as discussed above, for fully turbulent flows the effect of viscosity become unimportant, hence the effect of Reynolds number becomes unimportant, hence the term in brackets is approximately constant in this case. Consequently, $\varepsilon$ is proportional to $u_{0}^{3} / L_{0}$; we can deduce this also directly from dimensional analysis by declaring $v$ not to be a parameter. Locally in the jet, this is proportional to $u(z)^{3} / D(z)$, where the diameter of the jet is the local characteristic length scale for the velocity gradient. Combining,

$$
t_{\text {mix }}^{3} \sim D(z)^{3} / u(z)^{3}
$$

Putting this in dimensionless form,

$$
t_{\text {mix }}^{3}=\left(\frac{L_{0} \widetilde{t}_{\text {mix }}}{u_{0}}\right)^{3} \sim \frac{L_{0}^{3} \widetilde{D}(\widetilde{z})^{3}}{u_{0}^{3} \widetilde{u}(\widetilde{z})^{3}} \quad \because \widetilde{t}_{\text {mix }} \sim\left(\frac{\widetilde{D}(\widetilde{z})}{\widetilde{u}(\widetilde{z})}\right)=\widetilde{t}_{\text {mix }}(\widetilde{z})
$$

That is, the result obtained by applying specific knowledge of the structure of the turbulence characterizing submerged fully-turbulent jets is anticipated by dimensional analysis at the higher level of the conservation of momentum, as of course it must. This is important because it illustrates the validity at some level of detail of the higher-level analysis based on the validity of the fundamental conservation laws, which of course apply even if details about the flow structure are not available or are too complex to estimate the consequence of the conservation law in detail.

Reverting to dimensional form,

$$
t_{\text {mix }}=\left(L_{0} / u_{0}\right) \tilde{t}_{\text {mix }}(\widetilde{z})
$$

That is, for systems of differing size and imposed velocity, so long as we maintain geometric similarity, the mixing time observed at constant $\widetilde{z}$ (observed at the same ratio of jet length compared to the characteristic length) scales as the characteristic hydrodynamic time $L_{0} / u_{0}$ : the time required to traverse the characteristic length $L_{0}$ at the characteristic velocity $u_{0}$.

The above analysis is instructive but its application is limited, even aside from the simplicity of the example analyzed. It is crucial to understand that, although this analysis reveals the dependence of the mixing time on length and time (velocity) scale, it does not determine the ratio $u_{0} t_{m i x} / L_{0}$ at either scale. The dimensionless mixing time must be measured or estimated at one scale by means other than this analysis. Given that, one can estimate the mixing time at a different scale, all else remaining the same.

\section{A.1.5 Dimensional Analysis of Empirical Information}

Where the phenomena involve processes too complex to describe usefully in terms of first principles, such as the conservation of mass, momentum, and energy, the nature of relationships among dependent variables, independent variables, and physical parameters can be deduced from dimensional analysis based on the physical dimensions length $(L)$, time $(t)$, mass $(M)$, and temperature $(T)$ for the systems of interest here. Physical quantities such as velocity, energy or work, force, and pressure, and parameters such as density, viscosity, specific heat, etc., are expressed as combinations of the physical dimensions. For example, velocity [=] L/t, work [=] ML ${ }^{2} / \mathrm{t}^{2}$, viscosity [=] M/Lt, density [=] M/L $\mathrm{L}^{3}$, specific heat (per 
mass) $[=] \mathrm{L}^{2} / \mathrm{t}^{2} \mathrm{~T}$, where "[=]" means "has units of." Suppose a dependent variable $y$ depends on a set of independent variables $x_{i}$ and a set of parameters $b_{j}$. Functionally, we write

$$
y=f\left(x_{i} ; b_{j}\right)
$$

Say the physical dimensions of $y$ are $L^{\alpha_{0}} t^{\beta_{0}} M^{\gamma_{0}} T^{\delta_{0}}$; then the function $\mathrm{f}$ must have the same units. Say the physical dimensions of the $x_{i}$ are $L^{\alpha_{i}} t^{\beta_{i}} M^{\gamma_{i}} T^{\delta_{i}}$ and of the $b_{j}$ are $L^{\alpha_{j}} t^{\beta_{j}} M^{\gamma_{j}} T^{\delta_{j}}$. Then the function $\mathrm{f}$ must be constructed of the independent variables and parameters combined to give the dimensions $L^{\alpha_{0}} t^{\beta_{0}} M^{\gamma_{0}} T^{\delta_{0}}$. This is done by grouping the $\mathrm{x}_{\mathrm{i}}$ and $\mathrm{b}_{\mathrm{j}}$ both to provide the desired dimensions for $f$ from some grouping of $x_{i}$ and $b_{j}$ and to cancel the dimensions of all $x_{i}$ and $b_{j}$ not in the dimensional grouping by combining them in dimensionless groups that can be the argument of dimensionless functions within the dimensional function $f$. For example, from the conservation of momentum we found

$$
\widetilde{\mathbf{u}}=\widetilde{\mathbf{u}}(\widetilde{\mathbf{x}}, \tilde{t}, \operatorname{Re})
$$

Without knowledge of the conservation law, we could have begun with

$$
\mathbf{u}=f\left(\mathbf{x}, t, \mu, \rho, u_{0}, L_{0}\right)
$$

To be dimensionally consistent, we could propose

$$
\mathbf{u}=\mathbf{f}\left(\mathbf{x}, t, \mu, \rho, u_{0}, L_{0}\right)=u_{0} \mathbf{g}\left(\mathbf{x}, t, \mu, \rho, u_{0}, L_{0}\right)
$$

where $\mathrm{u}_{0}$ provides the dimensions of $\mathbf{f}$, and hence $\mathbf{g}$ is a dimensionless vector function and must have dimensionless arguments. Alternatively, we can make the entire form dimensionless by proposing

$$
\widetilde{\mathbf{u}}=\frac{\mathbf{u}}{u_{0}}=\mathbf{g}\left(\mathbf{x}, t, \mu, \rho, u_{0}, L_{0}\right)
$$

That is, we form a dimensionless group from the dependent variable and make it a dimensionless function of dimensionless groups of the $x_{i}$ and $b_{j}$. A simple algorithm for this is to assume the product form

$$
y=\prod_{i}^{\substack{\text { inpedendent } \\ \text { variables }}} x_{i}^{q_{i}} \prod_{j}^{\text {parameters }} b_{j}^{q_{j}}
$$

where the constants $\mathrm{q}_{\mathrm{i}}$ and $\mathrm{q}_{\mathrm{j}}$ are to be determined. Considering only the physical dimensions,

$$
L^{\alpha_{0}} t^{\beta_{0}} M^{\gamma_{0}} T^{\delta_{0}}=\prod_{i}^{\substack{\text { inpedendent } \\ \text { variables }}}\left(L^{\alpha_{i}} t^{\beta_{i}} M^{\gamma_{i}} T^{\delta_{i}}\right)^{q_{i}} \prod_{j}^{\text {parameters }}\left(L^{\alpha_{j}} t^{\beta_{j}} M^{\gamma_{j}} T^{\delta_{j}}\right)^{q_{j}}
$$


This is satisfied only if

$$
\begin{aligned}
& \alpha_{0}=\sum_{i}^{\substack{\text { independent } \\
\text { variables }}} q_{i} \alpha_{i}+\sum_{j}^{\text {parameters }} q_{j} \alpha_{j} \quad \beta_{0}=\sum_{i}^{\substack{\text { independent } \\
\text { variables }}} q_{i} \beta_{i}+\sum_{j}^{\text {parameters }} q_{j} \beta_{j} \\
& \gamma_{0}=\sum_{i}^{\substack{\text { independent } \\
\text { variables }}} q_{i} \gamma_{i}+\sum_{j}^{\substack{\text { parameters } \\
\text { var }}} \gamma_{j} \quad \delta_{0}=\sum_{i}^{\substack{\text { independent } \\
\text { variables }}} q_{i} \delta_{i}+\sum_{j}^{\text {parameters }} q_{j} \delta_{j}
\end{aligned}
$$

From this we find the $q_{i}$ and $q_{j}$ in terms of the $\alpha, \beta, \gamma$, and $\delta$ characterizing the physical dimensions of the $x$ and $b$. Usually, these four equations are insufficient to determine all of the $q$, which hence remain arbitrary. However, these constraints serve to define dimensionless groups that are admissible as arguments of the dimensionless function $g$, as is illustrated below.

Returning to the example and applying this algorithm, we proceed by proposing

$$
\mathbf{u}=\mathbf{f}\left(\mathbf{x}^{a}, t^{b}, \mu^{c}, \rho^{d}, u_{0}^{e}, L_{0}^{f}\right)
$$

where we have reverted to non-subscripted nomenclature more convenient to a specific example. In terms of physical dimensions,

$$
\frac{L}{t}=f\left(L^{a}, t^{b},\left(\frac{M}{L t}\right)^{c},\left(\frac{M}{L^{3}}\right)^{d},\left(\frac{L}{t}\right)^{e}, L^{f}\right)
$$

Then

$$
\begin{aligned}
1 & =a-c-3 d+e+f \\
-1 & =-b-c-e \\
0 & =c+d
\end{aligned}
$$

where units of temperature do not appear in this case. Given these six unknowns and three constraining equations, obviously all we can do is find three of the unknowns in terms of three others. Which three to choose is arbitrary; hence, there are multiple, equally valid solutions. However, usually some choices are preferable for various reasons, and we are free to choose them. In this case, one solution is $a=b-c-$ $f, d=-c$, and $e=1-b-c$, corresponding to

$$
\frac{\mathbf{u}}{u_{0}}=\mathbf{g}\left(\mathbf{x}^{b-c-f} t^{b} \mu^{c} \rho^{-c} u_{0}^{-b-c}\right)=\mathbf{g}\left(\left(\frac{\mathbf{x} t}{u_{0}}\right)^{b}\left(\frac{\mu}{\rho \mathbf{x} u_{0}}\right)^{c}\left(\frac{\mathbf{x}}{L_{0}}\right)^{f}\right)
$$

This is easily rearranged to obtain the mathematically identical form

$$
\frac{\mathbf{u}}{u_{0}}=\mathbf{g}\left(\left(\frac{L_{0} t}{u_{0}}\right)^{b}\left(\frac{\mathbf{x}}{L_{0}}\right)^{b}\left(\frac{\mu}{\rho L_{0} u_{0}}\right)^{c}\left(\frac{\mathbf{x}}{L_{0}}\right)^{-c}\left(\frac{\mathbf{x}}{L_{0}}\right)^{f}\right)=\mathbf{g}\left(\left(\frac{\mathbf{x}}{L_{0}}\right)^{f+b-c}\left(\frac{L_{0} t}{u_{0}}\right)^{b}\left(\frac{\mu}{\rho L_{0} u_{0}}\right)^{c}\right)
$$


to find that we can express the effect of position and time through the dimensionless position and time developed by dimensional analysis of conservation laws. Recall that the result serves to identify the dimensional and dimensionless groups admissible as arguments of the function $f$. The form of the function is not determined by dimensional analysis; hence, deduction is limited to the functional dependence in terms of dimensionless groups, which in this example is

$$
\frac{\mathbf{u}}{u_{0}}=\mathbf{g}\left(\left(\frac{\mathbf{x}}{L_{0}}\right),\left(\frac{L_{0} t}{u_{0}}\right),\left(\frac{\mu}{\rho L_{0} u_{0}}\right)\right)
$$

Using the notation of dimensionless variables introduced above, $\widetilde{\mathbf{u}}=\mathbf{g}(\widetilde{\mathbf{x}}, \widetilde{t}, \mathrm{Re})$, which, as it must be, is identical to the result obtained starting with the conservation of momentum because, for this simple case, from that expression we deduced the relationship among all the variables and parameters for this simple case.

Suppose that the fluid is a yield stress fluid. Then the same dimensional analysis leads to

$$
\widetilde{u}=\left(\widetilde{\mathbf{x}}, \tilde{t}, \operatorname{Re}, \operatorname{Re}_{\tau}\right)
$$

where $\mathrm{Re}_{\tau}$ is the yield Reynolds number: $\operatorname{Re}_{\tau}=\rho u_{0}^{2} / \tau_{0}$ where, as noted above, $\tau_{0}$ is the yield stress.

\section{A.1.6 Characteristic Times}

\section{A.1.6.1 Hydrodynamic Times}

There are two related characteristic hydrodynamic time scales. The first is a linear residence time: a characteristic length $L_{0}$ divided by a characteristic velocity $u_{0}$; i.e., the time "in residence" while traversing a characteristic length at a characteristic velocity. This is also the residence time for "plug flow" through a volume. The second is a volumetric residence time: the time "in residence" in a characteristic volume at a characteristic volumetric flow rate. The linear time scales as $L_{0} / u_{0}$, the volumetric time scales as $L_{0}{ }^{3} /\left(L_{0}{ }^{2} u_{0}\right)=L_{0} / u_{0}$. That is, they scale the same with length and velocity. If a liner residence time is matched between two geometrically similar systems, then so is a volumetric residence time. Therefore, the characteristic time for continuous hydrodynamic flows is

$$
t=\frac{L}{u}
$$

\section{A.1.6.2 Process Times}

Because the PEP and WTP use pulsed mixing in addition to the characteristic hydrodynamic time scales based on jet velocities, there are characteristic times based on the pulse cycle. There are the pulse interval (cycle length), $t_{P}$, and the pulse duration (drive time), $t_{D}$. 


\section{A.1.6.3 Mass Balance Times}

Mass balances describing the rates of changes of concentrations in the PEP and WTP have the general form

$$
V \frac{d C}{d t}=F_{\text {in }} C_{\text {in }}-F_{\text {out }} C
$$

where $\quad \mathrm{V}=$ volume of the system undergoing a change in concentration

$\mathrm{C}=$ mass concentration

$\mathrm{F}_{\text {in }}$ and $\mathrm{F}_{\text {out }}=$ volumetric flow rates into and out of the system

$\mathrm{C}_{\mathrm{in}}=$ concentration in the inlet flow.

Then, if, for the sake of simplicity, the flow rates, densities, and hence the volume are considered to be constant,

$$
\frac{d C}{d\left(t / t_{0}\right)}=-\left(C-\frac{F_{\text {in }}}{F_{\text {out }}} C_{\text {in }}\right) \quad \therefore d \ln \chi=-d\left(t / t_{0}\right) \quad \chi=C-\frac{F_{\text {in }}}{F_{\text {out }}} C_{\text {in }} \quad t_{0}=V / F_{\text {out }}
$$

Then

$$
C=C\left[\frac{F_{\text {in }}}{F_{\text {out }}} C_{\text {in }},\left(t / t_{0}\right)\right]
$$

That is, the concentration changes over time relative to the characteristic time constant $t_{0}$, which is characteristic time for the mass balance. For a characteristic length scale $\mathrm{L}$ and characteristic velocity $\mathrm{u}$, then $t_{0}$ scales as $\left(L^{3}\right) /\left(L^{2} u\right)$ or $L / u$, the corresponding hydrodynamic residence time, everything else being equal.

The above is obtained when geometric similarity is preserved. This is intentionally not true for the filtration unit operation (described in Section 6.2). The filtration area of the PEP is sized relative to the WTP, not to preserve geometric similarity, but such that the flow rate ( $F_{\text {out }}$, as treated here) makes $t_{0}$ for filtration the same between the PEP and WTP.

\section{A.1.6.4 Chemical Reaction Times}

The characteristic time for leaching is developed in some detail in Section A.2.2.1. In very general terms, this time depends on a rate law for the leaching chemical reaction:

$$
\frac{d X}{d t}=f\left(X, T, C_{0}\right)
$$


where $\mathrm{X}$ is conversion, $\mathrm{T}$ is temperature, and $\mathrm{C}_{0}$ is the pre-reaction concentration of solid reactant in the slurry. This is much simplified but sufficient to identify the corresponding characteristic time.

Integration and inversion gives

$$
t_{X}=\int_{0}^{X} \frac{d X}{f\left(X, T, C_{0}\right)}=F\left(X, T, C_{0}\right)
$$

where $t_{X}$ is the time required to attain a specified conversion, which depends on attributes that are independent of the size of the system.

\section{A.1.6.5 Heat Transfer Times}

Finite time is required to heat or cool a mass over a specified temperature difference. In the absence of significant evaporative heat loss, neglecting the power of mixing processes and lumping together all heat losses to the surroundings, the transient heat balance for a vessel is

$$
\rho V C_{p} \frac{d T}{d t}=\dot{m}_{s} \Delta H^{v a p}-A_{x} h_{x}\left(T-T_{x}\right)-A_{a} h_{a}\left(T-T_{a}\right)+\Delta \mathrm{H}_{\mathrm{rxn}} \mathrm{r}_{\mathrm{rxn}}
$$

where

$$
\begin{aligned}
\rho & =\text { average density } \\
V & =\text { volume of the system } \\
\mathrm{C}_{\mathrm{p}} & =\text { average specific heat } \\
\mathrm{T} & =\text { average temperature of the system } \\
\dot{m}_{s} & =\text { mass rate of addition of steam } \\
\Delta \mathrm{H}^{\mathrm{vap}} & =\text { heat of vaporization for the steam } \\
\mathrm{A}_{\mathrm{x}} & =\text { area of cooling heat exchanger } \\
\mathrm{h}_{\mathrm{x}} & =\text { heat transfer coefficient in cooling heat exchanger } \\
\mathrm{T}_{\mathrm{x}} & =\text { temperature of coolant in the cooling heat exchanger } \\
\mathrm{A}_{\mathrm{a}} & =\text { area of heat exchange to the surroundings } \\
\mathrm{h}_{\mathrm{a}} & =\text { composite ambient heat transfer coefficient } \\
\mathrm{T}_{\mathrm{a}} & =\text { ambient temperature } \\
\Delta \mathrm{H}_{\mathrm{rxn}} & =\text { heat of reaction when dissolving or leaching species } \\
\mathrm{T}_{\mathrm{rxn}} & =\text { corresponding rate of reaction }
\end{aligned}
$$

When the heat of reaction is important compared to vaporization and condensation, integration over time is complex and becomes a significant technical exercise. If not, then integration over time gives:

$$
\Delta T(t)=\Delta T(0) e^{-t / t_{*}} \Delta T(t)=T(t)-T_{*}-t_{*} \dot{T}_{*} \quad t_{*}=\frac{\rho V C_{p}}{A_{x} h_{x}+A_{a} h_{a}} \quad T_{*}=\frac{A_{x} h_{x} T_{x}+A_{a} h_{a} T_{a}}{A_{x} h_{x}+A_{a} h_{a}} \quad \dot{T}_{*}=\frac{\dot{m}_{s} \Delta H^{v a p}}{\rho V C_{p}}(
$$


The time-constant $t *$ scales as $L$; the temperature $T *$ is independent of scale; the temperature rate $\dot{T}_{*}$ scales as $\dot{m}_{s} / L^{3}$; and the product $t_{*} \dot{T}_{*}$ scales as $\dot{m}_{s} / L^{2}$. If there is no forced cooling, i.e., $A_{x} h_{x}=0$, then during steam heating, the ambient heat loss will be small enough that $t / t_{*}<<1$. Then

$$
T(t) \doteq T(0)+\frac{t}{t_{* *}} \quad t_{* *}=\frac{\frac{\rho V C_{p}}{\dot{m}_{s} \Delta H^{v a p}}}{1+\frac{A_{a} h_{a}}{\dot{m}_{s} \Delta H^{v a p}}\left(T_{a}-T(0)\right)}
$$

where $t_{* *}$ is the characteristic time constant for heating with simultaneous ambient heat loss. That is, so long as the rate of steam heating is such that $\dot{m}_{s} \Delta H^{v a p}<<A_{a} h_{a}$, the characteristic heating time is independent of the rate of ambient heat loss and scales as $L^{3} / \dot{m}_{s}$. For the case of forced cooling with simultaneous ambient heat loss where the rate of forced cooling is much faster than the rate of ambient heat loss, then

$$
T(t)-T_{x}=\left(T(0)-T_{x}\right) e^{-t / t_{*}} \quad t_{*}=\frac{\rho V C_{p}}{A_{x} h_{x}}
$$

and the characteristic time for cooling scales as $L$.

\section{A.2 Key Mass Balances}

\section{A.2.1 Wash Factors}

Washing separates dissolved components from solids in two steps: first, separating dissolved from solid components in the feed ("concentration"), and second, adding water to the remaining solids to replace interstitial liquid with condensate ("replace liquid"), which serves also to dissolve any soluble solids in the slurry. Water is added as condensate collected from process operations.

The first step — concentration - is accomplished simply by removing liquid from the tank holding the slurry, whether the solid phase is suspended as a slurry or residing en masse on the floor of the tank. This step is inherently efficient and unaffected by the scale of this system other than affects on the filtration rate, as discussed above.

The second step - replace liquid — consists of replacing interstitial fluid among solid particles by condensate, and potentially additionally consists of dissolving soluble solids among the solid particles. Although condensate is not added continuously, we treat this approximately as though condensate is added and filtrate is removed continuously at volumetrically equal rates, such that the volume of the liquid phase remains constant. The replacement step is affected potentially by the uniformity in the distribution of the added condensate over the interstitial liquid volume of the slurry, and the uniformity in the distribution of the solids mass over the volume of the slurry. The first affects the local fraction of interstitial liquid replaced by condensate; the second affects the fraction of any soluble solids locally dissolved by the added condensate. 
Thus, there are two phenomena to consider: mixing of the liquid phase over the volume of the slurry, and dispersion of the solids uniformly over the liquid phase. Mixing the liquid phase depends on the motion of the slurry, including the particles, where the particles affect the bulk density of the slurry and its rheological properties and hence affect the mixing. The particles are dispersed over the volume of the slurry by the turbulent motion of the slurry, confounded by settling of particles relative to the liquid, which is driven by gravity and the density of the particles relative to the liquid phase. Hence, both are hydrodynamic processes, and both depend on density differences: at the scale of the tank for mixing the slurry, and, for dispersion of the particles, at the both the scale of the tank and the scale of particles.

If no soluble solids exist in the waste, then washing is simply replacement of interstitial liquid. If soluble solids do exist, then the interstitial liquid is saturated with respect to those solids, and washing includes both dilution of the interstitial liquid by replacing it and dissolution of soluble solids into the diluted interstitial fluid. Assuming the dissolution is fast, then the interstitial liquid remains saturated during dilution until the soluble solids are dissolved entirely. This is depicted in Figure A.3 for a hypothetical slurry consisting of an insoluble solid and a soluble solid initially coexisting in solid and dissolved forms in water. "Insoluble solid" denotes a solid with a solubility small enough that only a negligible fraction can be dissolved by diluting the liquid phase with water. "Soluble solid" denotes a solid with a solubility low enough that it can coexist with its solid phase in the waste, but large enough that it can be completely dissolved by diluting the waste with concentrate.
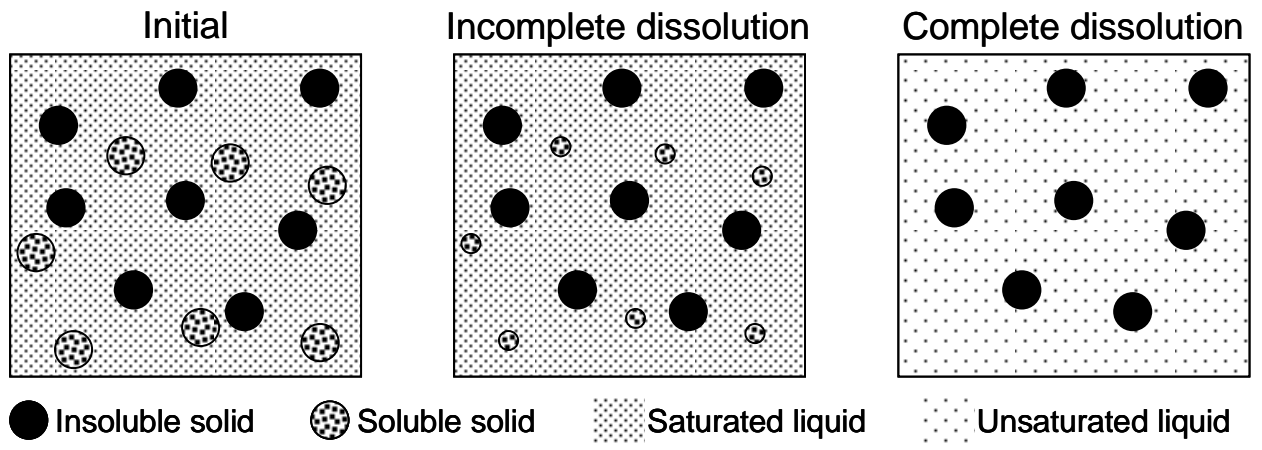

Figure A.3. Dissolution of Soluble Solids by Dilution of Interstitial Liquid

The intended process would add enough condensate to dissolve the total mass of soluble solids while replacing interstitial liquid with condensate uniformly over the slurry volume. The left of Figure A.3 depicts the initial case of undissolved soluble solids waste after concentrating the waste. The liquid is saturated. The center depicts the intended state following the "replacement" step in Figure 3.3 of the main report, where the liquid volume replaced locally by condensate is insufficient to dissolve all the local soluble solids. This can be either because, although the solids are distributed uniformly throughout the liquid, the total condensate added is insufficient to dissolve the total mass of soluble solids, or because, although the total condensate added is sufficient to dissolve the total mass of soluble solids, the solids are not distributed uniformly and locally more soluble solids exist than can be dissolved by uniform dilution of interstitial liquid.

As is noted in Section 7.3 of the main report, we will infer from PEP data the extent and nature of non-uniform distribution of the solids or of the distribution of condensate in replacing interstitial fluid. Given the simple conceptual model of non-ideal mixing imposed here, we would infer values of $\alpha$ and $\beta$ to determine if they can be statistically distinguished from zero-i.e., distinguished from ideal mixing. 
Analysis of PEP data may suggest alternative conceptual models, in which case they will be imposed and invoked through mass balances analogous to the development leading to a characterization in terms of $\alpha$ and $\beta$.

\section{A.2.1.1 Design Basis Wash Factors}

If there are no soluble solids in the waste, the mass of a soluble species in the waste is

$$
V_{0}\left(1-\phi_{\text {total }}\right) C_{0}
$$

where $\mathrm{V}_{0}$ is the volume of waste being processed, $\varphi_{\text {total }}$ is the volume fraction of all solids present, and $\mathrm{C}_{0}$ is the concentration of the soluble species in the liquid phase of the waste. After the concentration step, the mass of soluble solids in the slurry is $V\left(1-\phi_{\text {total }}\right) C_{0}$, where $V$ is the volume of slurry in the process tank. If the liquid phase remains well mixed, a mass balance over the liquid phase of the slurry volume during replacement of interstitial fluid by condensate is

$$
V d C=-C d V_{L}=-C d V_{C}
$$

where $C$ is the concentration of the soluble species in the liquid phase and we assume $d V_{L}$ and $d V_{C}$, the volumes of liquid lost by filtration and the volume of condensate added, are the same because condensate is added to maintain a constant slurry volume. Integrating over the replacement step,

$$
C=C_{0} e^{-\Delta V_{C} / V}
$$

$\Delta V_{C}$ is the volume of condensate added. The mass of soluble species at the end of the wash process is

$$
m_{\text {end }}=V C=V C_{0} e^{-\Delta V_{C} / V}
$$

The wash factor $\omega$ is the ratio of the mass of the soluble species removed to the mass in the waste, which is

$$
\omega=1-\frac{m_{\text {end }}}{m_{\text {initial }}}=1-\frac{V C_{0} e^{-\Delta V_{C} / V}}{V_{0} C_{0}}=1-\left(V / V_{0}\right) e^{-\left(\Delta V_{C} / V\right)}
$$

Thus, the wash factor depends both on the ratio of the concentration ratio $V_{0} / V$ and the dilution ratio $\Delta V_{C} / V$.

If instead there are soluble solids in the waste, the wash factor is based on the total initial mass soluble solids removed, including the mass of the dissolved solid saturating the liquid phase. If the volume fraction of soluble solids $\varphi_{S}$ is initially $\varphi_{S 0}$ in the waste, the volume fraction of soluble solids after the "concentrate solids" step is

$$
\phi_{S}=V_{S} / V=\left(V_{0} / V\right) \phi_{S 0}
$$

That is, the volume fraction of soluble solids after concentration is the fraction in the waste multiplied by the concentration ratio. This occurs by separating liquid from solids at a constant liquid composition, 
and hence, no dissolution or precipitation of soluble solids occurs. If the saturation concentration in the liquid is $C_{e q}$, then the mass of the soluble solid initially in the liquid phase in the waste is

$$
m_{S 0, L}=V_{L} C_{e q}=V_{0}\left(1-\phi_{S 0}-\phi_{I 0}\right) C_{e q}
$$

where $\varphi_{I 0}$ is the initial volume fraction of insoluble solids in the waste, and the mass of soluble solid initially in the solid phase is

$$
m_{S 0, S}=\rho_{S} \phi_{S 0} V_{0}
$$

where $\rho_{S}$ is the density of the soluble solids. Hence, the initial total mass of the soluble component is

$$
m_{S 0}=V_{0}\left(1-\phi_{S 0}-\phi_{I 0}\right) C_{e q}+\rho_{S} \phi_{S 0} V_{0}
$$

The volume of liquid removed during the "concentrate solids" is the volume of condensate added, which, from the definition of $V_{0}$, is

$$
\Delta V_{C}=V_{0}-V
$$

Therefore, the mass of the soluble solid removed during concentration of solids is

$$
\Delta m_{S 1}=\Delta V_{L} C_{e q}=\left(V_{0}-V\right) C_{e q}
$$

Addition of condensate first dissolves any soluble solids while dissolution maintains the saturation concentration in the liquid, and then dilutes the liquid phase after dissolution is complete. The minimum condensate required to dissolve the soluble solids is found from

$$
C_{e q} \Delta V_{C, \min }=m_{S, 0}
$$

Then dilution of the interstitial liquid after dissolution is complete results in a concentration

$$
C=C_{e q} e^{-\left(\Delta V_{C}-\Delta V_{C, \text { min }}\right) / V}
$$

Hence, the mass of dissolved soluble solids remaining after the wash process is

$$
m_{\text {end }}=V C=V C_{e q} e^{-\left(\Delta V_{C}-\Delta V_{C, \min }\right) / V}
$$

Then the wash factor is

$$
\begin{aligned}
& \omega=\frac{m_{S, 0}-m_{e n d}}{m_{S, 0}}=1-\frac{V C_{e q} e^{-\left(\Delta V_{C}-\Delta V_{C, \min }\right) / V}}{V_{0}\left(1-\phi_{S 0}-\phi_{I 0}\right) C_{e q}+\rho_{S} \phi_{S 0} V_{0}} \\
& =1-\frac{1}{\left(V_{0} / V\right)} \frac{\left(C_{e q} / \rho_{S}\right) e^{-\left(\Delta V_{C}-\Delta V_{C, \min }\right) / V}}{\left(1-\phi_{S 0}-\phi_{I 0}\right)\left(C_{e q} / \rho_{S}\right)+\phi_{S 0}}
\end{aligned}
$$


Denote the condensate ratio as $\lambda=\frac{\Delta V_{C}}{V}$ and also $\lambda_{\min }=\frac{\Delta V_{C, \min }}{V}$, the concentration ratio as $\psi=\frac{V_{0}}{V}$, and the dimensionless initial concentration as $\chi_{0}=\frac{C_{0}}{\rho_{S}}$ which is $\frac{C_{e q}}{\rho_{S}}$ if soluble solids exist in the waste. Then the wash factor can be written as

$$
\omega=1-\frac{1}{\psi} \frac{\chi_{0} e^{-\left(\lambda-\lambda_{\min }\right)}}{\left(1-\phi_{S 0}-\phi_{I 0}\right) \chi_{0}+\phi_{S 0}}
$$

If no soluble solids exist in the waste, then $\lambda_{\min }$ and $\varphi_{S 0}$ are both zero.

The property $\chi_{0}$ and the volume fractions in the waste depend on the waste being processed but not on the scale of the system processing the waste. The ratio $\psi$ is a ratio of volumes. The ratios $\lambda$ and $\lambda_{\min }$ are also ratios of volumes. They can be matched between systems as an operational specification. Therefore, either for the case with or without soluble solids in the waste, nominally the wash factor does not depend on the scale of the system. However, the assumptions implicit to this conclusion are that the condensate added replaces interstitial liquid uniformly over the volume of the slurry in a tank, and that soluble solids are volumetrically distributed uniformly of the volume of the slurry in a tank. That is, we have assumed two characteristics of ideal mixing.

\section{A.2.1.2 Effect of Systematic Non-Uniformity}

As noted in the External Flowsheet Review Team (EFRT) critique, ideal mixing is not ensured. Thus, we need to consider the effects of non-ideal mixing.

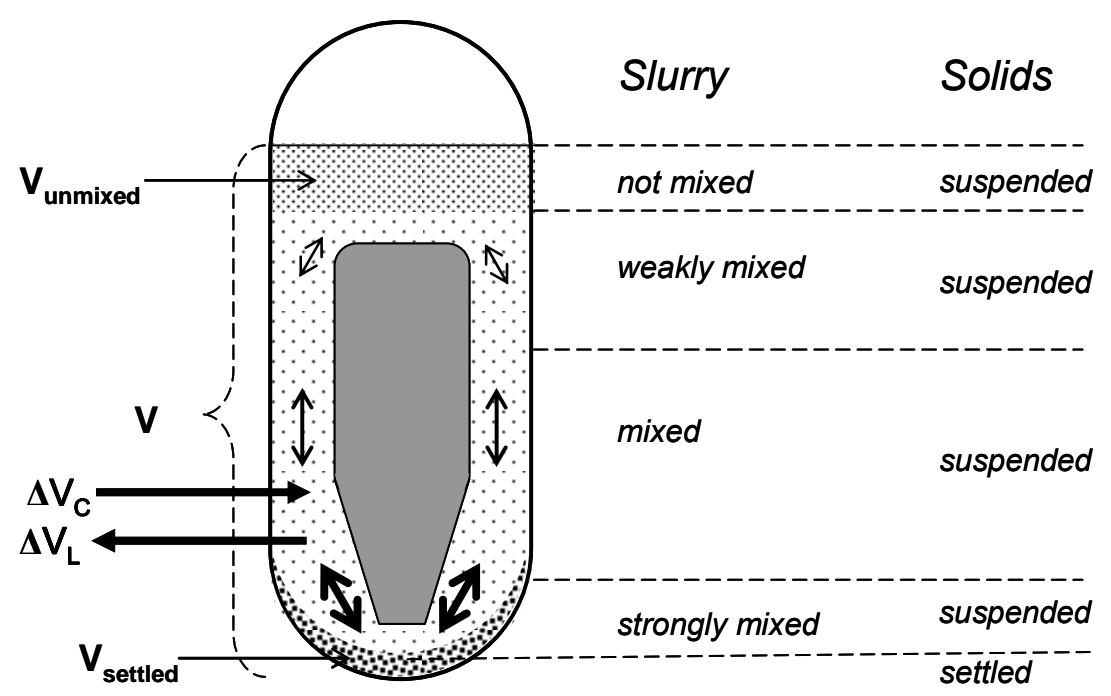

Figure A.4. Conceptual Settling and Imperfect Mixing During Washing

Complex situations potentially interfere with ideal mixing. Two extreme possibilities are depicted in Figure A.4: settling of solids to form a dense, stagnant layer on the floor of the tank, and failure of mixing to penetrate the upper region of the slurry, where the solids volume fraction in the unmixed region might not differ from the rest of the slurry but the fraction of the inlet condensate distributed to this region 
is less than for the rest of the slurry. The solids void fraction in the settled region is much greater than for the rest of the slurry and impedes fluid motion sufficiently to prevent replacement of the interstitial liquid by condensate mixing with the rest of the slurry. We assume that it also impedes suspension of the solids in spite of strong mixing of the adjacent slurry. We continue to assume that the volume $\Delta V_{L}$ of liquid removed by filtration and volume $\Delta V_{C}$ of condensate added are equal, simultaneous, and continuous.

As noted above, the mass of soluble species removed during the concentration step is unaffected by settled solids, and this remains true even if part of the liquid is unmixed. However, the unmixed region affects the mass balance over the liquid phase during the addition of condensate with removal of filtrate. The calculated mass of soluble species at the end of the wash process becomes

$$
m_{\text {end }}=m_{\text {settled }}+V_{\text {unmixed }} C_{0}+\left(V-V_{\text {unmixed }}\right) C_{0} e^{-\Delta V_{C} /\left(V-V_{\text {unmixed }}\right)}
$$

where $C_{0}=C_{e q}$ if soluble solids are present in the waste. Then the wash factor is

$$
\omega_{\text {unmixed }, \text { settled }}=1-\frac{m_{\text {settled }}+V_{\text {unmixed }} C_{0}+\left(V-V_{\text {unmixed }}\right) C_{0} e^{-\left(\Delta V_{C}-\Delta V_{C, \min }\right) /\left(V-V_{\text {unnixed }}\right)}}{V_{0}\left(1-\phi_{S 0}-\phi_{I 0}\right) C_{0}+\rho_{S} \phi_{S 0} V_{0}}
$$

Denoting $\alpha=V_{\text {unmixed }} / V$ and $\beta=V_{\text {settled }} / V$ and rearranging,

$$
\omega_{\text {unmixed }, \text { settled }}=1-\frac{1}{\psi} \frac{\beta \phi_{S, \text { settled }}+\alpha \chi_{0}+(1-\alpha) \chi_{0} e^{-\frac{\lambda-\lambda_{\min }}{1-\alpha}}}{\left(1-\phi_{S 0}-\phi_{I 0}\right) \chi_{0}+\phi_{S 0}}
$$

If solids settle, the solids volume fraction of the settled region depends on the solids in the system, which can be matched between systems as an operational specification. However, this model of the effect of non-ideal mixing introduces two additional parameters $(\alpha$ and $\beta)$ that cannot be specified directly as operational parameters, but which instead are determined by hydrodynamic phenomena that depend on the size of the system, the velocities, and the properties of the materials being processed.

If for some component

$$
\frac{\Delta V_{C}}{V}=\lambda<\lambda_{\min }=\frac{m_{S, 0}}{C_{e q} V}
$$

then the mass of soluble solids remaining after the wash process is

and the wash factor is

$$
m_{\text {end }}=\rho_{S} \phi_{S 0} V_{0}-C_{e q} \Delta V_{C}
$$

$$
\omega_{\lambda<\lambda_{\min }}=\frac{1-\frac{C_{e q}}{\rho_{S}} \frac{\Delta V_{C}}{\phi_{S 0} V_{0}}}{1+\frac{1-\phi_{S 0}-\phi_{I 0}}{\phi_{S 0}} \frac{C_{e q}}{\rho_{S}}}=\frac{\phi_{S 0}-(\lambda / \psi) \chi_{0}}{\phi_{S 0}\left(1-\chi_{0}\right)+\left(1-\phi_{I 0}\right) \chi_{0}}
$$


Note that this results even if the solids or the condensate are not distributed uniformly over the liquid, so long as locally $\lambda<\lambda_{\min }=m_{S, 0} / C_{e q} V$. Because the solubility $C_{e q}$ tends to vary by orders of magnitude from component to component, likely either $\lambda<<\lambda_{\min }$ or $\lambda>>\lambda_{\min }$ for a given component. Thus, even if the solids mass concentration $d m_{S, O} / d V$ locally differs from that averaged over the tank, $m_{S, 0} / V$, it is unlikely that locally $\lambda \sim \lambda_{\min }$, and hence no attempt is made here to quantify the effect of random, modest variations of the mass concentration to the wash factor of a specific component.

\section{A.2.2 Leach Factors}

Leaching is a chemical conversion of insoluble solids to a soluble form followed by separation of the dissolved soluble form from residual solids. The first part is dewatering and washing: concentration of the residual solids by removing saturated liquid and potentially addition of condensate to promote further dissolution of the converted solid form if not already completely dissolved. The leach factors are the mass fraction of the initial mass of the target insoluble solid that is removed from the waste by filtration. This is determined by the fraction of the solid converted to the soluble form, the fraction of the soluble form dissolved, and the fraction of the dissolved soluble form removed as filtrate. Consequently, leach factors are a combination of the chemical conversion and the wash factor for the dissolved form.

\section{A.2.2.1 Design Basis Leach Factors}

For the case of leaching of aluminum components, two chemical reactions occur: conversion of Gibbsite by caustic to the dissolved form

$$
\mathrm{Al}(\mathrm{OH})_{3(s)}+\mathrm{OH}^{-}(a q)=\mathrm{Al}(\mathrm{OH})_{4}^{-}(a q)
$$

and conversion of Boehmite by caustic to the dissolved form

$$
\mathrm{AlO}(\mathrm{OH})_{(s)}+\mathrm{OH}_{(a q)}^{-}+\mathrm{H}_{2} \mathrm{O}_{(a q)}=\mathrm{Al}(\mathrm{OH})_{4}^{-}(a q)
$$

The reactions are reversible; both dissolution and precipitation can occur within process times.

If leaching occurs at or near $100^{\circ} \mathrm{C}$, the solubility of Gibbsite is great enough that the solids dissolve essentially completely. The rate of dissolution is slow at room temperature but is very fast at the near boiling conditions of the PTF Al leaching process, and essentially the Gibbsite dissolves before the leaching temperature is reached and significant leaching of Boehmite begins.

At the process conditions, the solubility of Boehmite is small enough that its dissolution is impeded by $\mathrm{Al}(\mathrm{OH})_{4}{ }^{-}$in solution. This includes that resulting from dissolution of Gibbsite as well as from Boehmite.

The dissolution of Gibbsite is approximately instantaneous and affects the resulting aluminate in the liquid slows the dissolution of Boehmite, and the dissolution of Boehmite is controlled by the rate of the chemical reaction, which depends strongly on temperature and is impeded by accumulation of the dissolved form in solution. 
For the case of leaching of chromium oxides, the reaction converts the oxides to very soluble forms and the reaction is essentially irreversible. It is also very fast, and the rate is limited mainly by the rate of availability of permanganate to the suspended solids.

Therefore, we can divide the leaching reactions into two categories: fast (hot, caustic leaching of Gibbsite, oxidative leaching of chromium oxide), where the leach factor is limited by the wash factor, and slow (hot, caustic leaching of Boehmite), where the leach factor is limited by the chemical conversion. Washing is discussed above. Here we discuss chemical conversion.

Based on laboratory experiments and the literature, the form of the reaction of Boehmite is ${ }^{1}$

$$
r=\frac{1}{A_{p}} \frac{d V_{p}}{d t}=r_{0}(T) f_{R}\left(C_{O H}{ }^{-}(X)\right)\left[1-f_{P}\left(C_{A l(O H)_{4}^{-}}(X)\right)\right]
$$

where $r$ is the reaction rate in terms of the rate of recession of the surface of the reacting particle (i.e., units of velocity), $A_{p}$ is the area of the particle, $V_{p}$ is the volume of the particle, $r_{0}(T)$ is a rate constant (length/time) that depends strongly on temperature, $f_{R}$ is a dimensionless function of the concentration in solution at the surface of the particle of the reagent $O H, f_{P}$ is a dimensionless function of the concentration in solution at the surface of the particle of the reaction product $\mathrm{Al}(\mathrm{OH})_{4}^{-}$. The area of the reacting particle is a function of the size, and the initial area and volume of the reacting particles vary by orders of magnitude over the particles and must be described by a particle size distribution function.

As is shown in Section A.2.2.3, the particles of interest are small enough that the concentrations at the surface of the particle will not differ substantially from concentrations averaged locally over the liquid in which the particles are embedded. Essentially, the resistance to mass transfer between this liquid and the surface of the particle is negligible. Therefore, the reaction rate does not depend on the size of the particle for a given composition of the liquid phase, but the rate of loss of particle mass decreases as the particles shrink and ultimately disappear because the reaction area decreases as the particles decrease in size and number.

Over the course of the reaction, the rate of loss of mass of reactive particles decreases for two reasons: the reaction area decreases, and the concentration of reaction products increases in the liquid phase, which decreases the reaction rate as described by the empirical function $f_{P}$.

Define the chemical conversion $X$ as

$$
X=\frac{m(0)-m(t)}{m(0)}
$$

where $m$ is the remaining mass of reactive solid and $m(0)$ is its initial value. Then

$$
\frac{d X}{d t}=-\frac{1}{m_{S, 0}} \frac{d m_{S}}{d t}=\left(\frac{\rho_{S}}{m_{S, 0}}\right)\left(-\frac{d V_{S}}{d t}\right)=\left(\frac{\rho_{S}}{m_{S, 0}}\right)\left(A_{S} r\right)
$$

\footnotetext{
${ }^{1}$ Peterson RA, GJ Lumetta, BM Rapko, and AP Poloxki. 2006. Modeling of Boehmite Leaching from Actual Hanford High-Level Waste Samples. PNNL-SA-51705, Pacific Northwest National Laboratory, Richland, Washington.
} 
where $A_{S}$ is the reactive area of the particles, which is a function of the particles sizes and the number of remaining particles, as is the mass of the particles, which can be expressed in terms of the conversion. Therefore, $A_{S}=A_{S}(X)$. Substituting,

$$
\frac{d X}{d t}=\frac{\rho_{S} A_{S}(X)}{m_{S, 0}} r
$$

Because $r$ depends on $f_{P}$, which depends on the concentration of the reaction product, which depends on the conversion and the local initial volume fraction of solids, $r=r\left(X, \varphi_{S 0}\right)$. Specifically, the concentration $C_{P}$ of the reaction product is related to the conversion by

$$
d C_{P}=\frac{d m_{P}}{V}=\frac{d m_{P}}{\left(-d m_{S}\right)} \frac{\left(-d m_{S}\right)}{V}=\frac{d m_{P}}{\left(-d m_{S}\right)} \frac{m_{S 0} d X}{V}=v_{P} \rho_{S} \phi_{S 0} d X
$$

where $v_{P}$ is the mass of reaction product produced per mass of solid converted. Actually, the liquid density depends on its composition, which is not constant. But typically, the effect is small. If the volume is constant, then integrating from the initial condition gives

$$
C_{P}\left(C_{P 0}, \phi_{S 0}, X\right)=C_{P 0}+v_{P} \rho_{S} \phi_{S 0} X
$$

Similarly, for the reagent concentration,

$$
C_{R}\left(C_{R 0}, \phi_{S 0}, X\right)=C_{R 0}+v_{R} \rho_{R} \phi_{S 0} X
$$

where

$$
v_{R}=\frac{d m_{R}}{d m_{S}} \text { and } \rho_{R} \text { is the density in the dissolved state of the reagent. }
$$

However, live steam is injected into the slurry to maintain the leaching temperature, thereby diluting the slurry with condensate during leaching. The rate of addition of condensate depends on the heat loss through the tank boundary, which occurs independently of the conversion. Consequently, the concentrations of reagents and reaction products actually are functions of both conversion and time. The analysis here proceeds as though the effect is, if not insignificant, at least not dramatic.

Proceeding, define a dimensionless reaction rate and reaction area as

$$
\tilde{r}=\frac{r(X)}{r(0)} \quad \tilde{A}(X)=\frac{A_{S}(X)}{A_{S}(0)}
$$

where

$$
r(0)=r_{0}(T) f_{R}\left(C_{\mathrm{OH}^{-}}(0)\right)\left(1-f_{P}\left(C_{A l(O H)_{4}^{-}}(0)\right)\right)
$$

Substituting,

$$
\frac{d X}{\widetilde{A}(X) \widetilde{r}(X)}=\frac{\rho_{S} A_{S}(0)}{m_{s, 0}} r(0) d t
$$


Integrating,

$$
F_{X}(X)=\int_{0}^{X} \frac{d X}{\widetilde{A}(X) \widetilde{r}\left(X, \phi_{S 0}\right)}=\frac{\rho_{S} A_{S}(0) r(0)}{m_{s, 0}} t=\tau\left(X, \phi_{S 0}\right)
$$

where

$$
\tau=\left(\rho_{S} A_{S}(0) r(0) / m_{s, 0}\right) t
$$

and the function $F_{X}(X)$ is determined by the functions $\widetilde{A}(X)$ and $\widetilde{r}(X)$. Thus, the time to reach a specific conversion is

$$
t(X)=\frac{m_{s, 0}}{\rho_{S} A_{S}(0) r(0)} \int_{0}^{X} \frac{d X}{\widetilde{A}(X) \widetilde{r}\left(X, \phi_{S 0}\right)}=\frac{1}{\rho_{S}\left(A_{S}(0) / m_{S, 0}\right)} \frac{1}{r(0)} \int_{0}^{X} \frac{d X}{\widetilde{A}(X) \widetilde{r}\left(X, \phi_{S 0}\right)}
$$

The ratio $\rho_{S} A_{S}(0) / m_{S, 0}$ of initial reactive area to initial volume of reactive solid must be measured in the laboratory for a specific waste. The initial rate $r(0)$ is a function of temperature and the initial concentrations of caustic and $\mathrm{Al}(\mathrm{OH})_{4}$, where the function must be developed from laboratory measurements of the leach rate of Boehmite, and the initial concentrations must be determined from process measurements and a mass balance.

Inverting the expression for $\tau\left(X, \phi_{S 0}\right)$,

$$
X=F_{X}^{-1}\left(F_{X}(X)\right)=F_{X}^{-1}\left(\frac{\rho_{S} A_{S}(0) r(0)}{m_{s, 0}} t\right)=F_{X}^{-1}(\tau)
$$

Thus,

$$
X=X\left(t, T(t), C_{\mathrm{R} 0}, C_{P 0}, \phi_{S 0}\right)
$$

where $t$ is time, $T(t)$ is temperature as a function of time, $C_{R 0}$ and $C_{P 0}$ are the initial concentrations in the liquid of the reagent $\left(\mathrm{OH}^{-}\right)$and reaction product $\left(\mathrm{Al}(\mathrm{OH})_{4}^{-}\right)$, and $\varphi_{S 0}$ is the initial value of the volume fraction of reactive solids. The functional form of $X$ is as developed above, which includes the reaction rate model, which must be determined from laboratory data.

As noted above, the reagent and reaction product concentrations change during leaching both due to changes in the dissolved masses due to the chemical conversion, and due to dilution by condensate from steam injected to overcome heat losses during leaching. Consequently, the reaction rate is a function of both conversion and, independently, time, and we have

$$
m_{s, 0} d X=\left[\rho_{S} A_{S}(0) r(0)\right] \widetilde{A}(X) \widetilde{r}(X, t) d t
$$

which is the implicit differential equation:

$$
\frac{d X / \widetilde{A}(X)}{d t}=\left(\frac{\rho_{S} A_{S}(0) r(0)}{m_{s, 0}}\right) \widetilde{r}(X, t)
$$


Given specific test conditions for either the PEP or the PTF, the expression can be integrated to predict the conversion as a function of time, but it cannot be put in a close form: the solution requires numerical integration of the differential equation. The error in not doing this can be estimated by completing the calculation of the conversion assuming constant volume, calculating the ending concentrations of the reagent and reaction products, calculating the corresponding ending reaction rate, compute the diluted values of the ending concentrations and re-computing the ending reaction rate, and comparing. If they are not nearly the same, the actual conversion can be estimated by solving the differential equation to find the design basis (uniform concentration) leach factors.

\section{A.2.2.2 Effect of Mass Transfer Resistance Around Reacting Particles}

The mass flux from the surface of a particle-i.e., the rate of mass loss per unit area, which is the reaction rate-is described by

$$
\left(\frac{d \dot{m}}{d A}\right)=\rho_{S} k f_{r}\left(C_{r, i}\right)\left[1-f_{p}\left(C_{p, i}\right)\right]
$$

where $k$ is a kinetic constant, and $f_{r}$ and $f_{p}$ are functions describing the dependence of the mass flux on the concentration $C_{p, i}$ of the product of the dissolution and the concentration $C_{r, i}$ of the reactant at the particle/liquid interface, denoted by the subscript " $i$." If the interfacial concentrations equaled the bulk concentrations, we would have

$$
\left(\frac{d \dot{m}}{d A}\right)=\left(\frac{d \dot{m}}{d A}\right)_{b}=\rho_{S} k f_{r}\left(C_{r, b}\right)\left[1-f_{p}\left(C_{p, b}\right)\right]
$$

where the subscript " $b$ " denotes the value far from the particle.

Expanding a Taylor series about this,

$$
\left(\frac{d \dot{m}}{d A}\right)_{i}=\left(\frac{d \dot{m}}{d A}\right)_{b}-\left\{\left[-\frac{d}{d C_{p, i}}\left(\frac{d \dot{m}}{d A}\right)\right]_{b}-\left[\frac{d}{d C_{r, i}}\left(\frac{d \dot{m}}{d A}\right)\right]_{b} \frac{C_{r, x}}{C_{p, x}} \frac{k_{p}}{k_{r}}\right\}\left(C_{p, i}-C_{p, b}\right)
$$

Where at steady state (relative to the relaxation time for the concentration profile around a microscopic particle), the concentration difference from the interface to the bulk is related between the product and the reactant by the equivalence of the reaction mass flux and the diffusion mass flux as follows.

$$
\left(\frac{d \dot{m}}{d A}\right)=\frac{\rho_{S}}{C_{A l, x}} k_{p}\left(C_{A l, i}-C_{A l, b}\right)=\frac{\rho_{S}}{C_{O H, x}} k_{r}\left(C_{O H, b}-C_{O H, i}\right)
$$

where $C_{p, x}$ and $C_{r, x}$ describe the mass of product produced or mass of reactant consumed per volume of particle reacted. Combining the linearized kinetic expression with the mass-transfer expression gives the concentration difference. 


$$
\left(C_{p, i}-C_{p, b}\right)=\frac{\left(\frac{d \dot{m}}{d A}\right)_{b}}{\frac{\rho_{S}}{C_{p, x}} k_{p}+k_{m}} \quad k_{m}=\left[-\frac{d}{d C_{p, i}}\left(\frac{d \dot{m}}{d A}\right)\right]_{b}+\left[\frac{d}{d C_{r, i}}\left(\frac{d \dot{m}}{d A}\right)\right]_{b} \frac{C_{p, x}}{C_{p, x}} \frac{k_{p}}{k_{r}}
$$

Substituting back into the mass-transfer expression gives the reaction flux relative to the value that would obtain in the absence of any mass-transfer resistance.

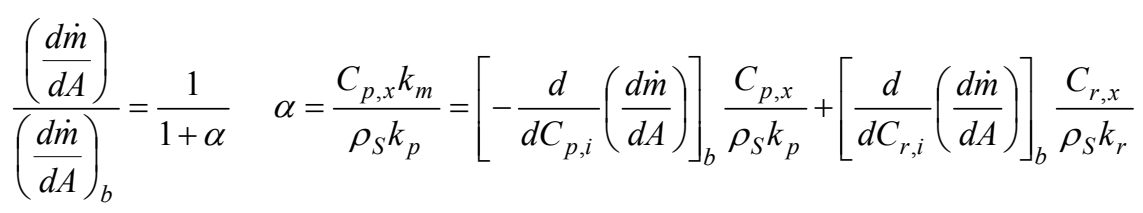

Hence, for $\alpha<<1$, the reaction rate is not affected by the mass-transfer resistance.

The above analysis requires knowledge of the chemical-reaction-rate expression for leaching. However, one can show mixing effects on mass transfer between waste particles, and the leach solution will not be important, based on mass-transfer and solubility arguments, as shown in the next section.

\section{A.2.2.3 Insignificance of Mass Transfer Resistance for Boehmite Dissolution}

One can evaluate the leach rate that would result if it were limited only by the mass-transfer resistance to determine if the resulting time constant for the conversion is consistent with measurements. As shown below, the time constant is short enough that any mass-transfer effect will not be important to interpreting PEP leach data, irrespective of the leach rate occurring in the absence of the masstransfer resistance.

The mass balance is

$$
\frac{d m}{d t}=-A(m) k_{C}\left(C_{e}-C_{b}\right)=-A(m) k_{C} \Delta C \quad \Delta C=\left(C_{e}-C_{b}\right) \approx C_{e}
$$

whereas before we considered the case of no product accumulation in the solution around the particles. The mass-transfer coefficient is

$$
\frac{k_{C} r}{D}=1+f(\operatorname{Re}, S c) \quad \therefore \frac{k_{C} r}{D} \geq 1 \quad \therefore k_{C} \geq \frac{D}{r}
$$

Combining,

$$
\frac{d m}{A(m)}=-\frac{D C_{e}}{r_{p}} d t
$$

Invoking the particle size

$$
m=\rho_{p} V_{p} \quad \therefore \frac{d m}{d t}=\rho_{p} \frac{d V_{p}}{d t}=\rho_{p} \frac{d V_{p}}{d r} \frac{d r}{d t}=\rho_{p} A(r) \frac{d r}{d t}
$$


which reduces to

$$
\rho_{p} A(r) \frac{d r}{d t}=-A(r) k_{C} C_{e}
$$

The process of separating, integrating, and rearranging gives the particle size vs time:

$$
r^{2}=r_{0}^{2}-\frac{2 D C_{e}}{\rho_{p}} t
$$

Then the total particle mass depends on time as

$$
m=\rho_{p} \frac{4 \pi}{3} r^{3}=\rho_{p} \frac{4 \pi}{3}\left(r_{0}^{2}-\frac{2 D C_{e}}{\rho_{p}} t\right)^{3 / 2}=\rho_{p} \frac{4 \pi}{3} r_{0}^{3}\left(1-\frac{2 D C_{e}}{r_{0}^{2} \rho_{p}} t\right)^{3 / 2}=m_{0}\left(1-\frac{2 D C_{e}}{r_{0}^{2} \rho_{p}} t\right)^{3 / 2}
$$

and the conversion is

$$
X=1-\frac{m}{m_{0}}=1-\left(1-\frac{2 D C_{e}}{r_{0}^{2} \rho_{p}} t\right)^{3 / 2}=1-\left(1-\frac{t}{t_{0}}\right)^{3 / 2} t_{0}=\frac{r_{0}^{2} \rho_{p}}{2 D C_{e}}
$$

We can estimate the order of magnitude of the concentration $\mathrm{C}_{\mathrm{e}}$ by noting that the order of magnitude of the solubility of $\mathrm{Al}$ in concentrated caustic is 1 molar (i.e., in the range 0.1 molar to 10 molar). Assuming this and evaluating, approximately,

$$
\widetilde{C}_{e} \sim 1 \mathrm{~mol} / \mathrm{L} \mathrm{Al}(\mathrm{OH})_{4}^{-}=1 \mathrm{~mol} / \mathrm{L} \mathrm{AlO}(\mathrm{OH}) \therefore C_{e} \sim \frac{60 \mathrm{~g} \mathrm{AlO}(\mathrm{OH})}{\mathrm{mol} \mathrm{AlO}(\mathrm{OH})} \frac{(1 \mathrm{~mol} / \mathrm{L})(0.001 \mathrm{~kg} / \mathrm{g})}{\left(0.001 \mathrm{~m}^{3} / \mathrm{L}\right)}=60 \frac{\mathrm{kg}}{\mathrm{m}^{3}}(\mathrm{Eq}
$$

Then the dissolution time constant $t_{0}$ is

$$
t_{0} \sim \frac{\left(10^{-5} \mathrm{~m}\right)^{2}\left(3000 \mathrm{~kg} / \mathrm{m}^{3}\right)}{2\left(10^{-9} \mathrm{~m}^{2} / \mathrm{s}\right)\left(60 \mathrm{~kg} / \mathrm{m}^{3}\right)}=5 \mathrm{sec}
$$

This compares to the dissolution time on the order of hours to days. Therefore, consistent with the evaluation of $\alpha$, the leach rate is orders-of-magnitude too small to be mass-transfer limited. The comparison of seconds to hours or days justifies the order-or-magnitude argument used in the approximate analysis. Further, although the above analysis is for the dissolution of Boehmite, the same order-of-magnitude will result for the oxidation of chromium species; where the reaction is essentially irreversible, the concentrations involved are not orders-of-magnitude different than for Boehmite dissolution, and the leach times are not orders-of-magnitude shorter.

Alternatively, we find that the effective particle size for which the mass transfer time constant approaches the observed leaching time constant would be $r_{0}\left(X^{1 / 2}\right)$, where $X$ is the ratio of leaching time constant to the mass transfer time constant. The value of $\mathrm{X}$ is on the order of hours to seconds-i.e., 3600 . Then the required particle size would be roughly 60 times greater in radius, or 216,000 time greater in volume, than observed. This might occur if primary particles form flocks, but the flocks would need to 
be very large. In that case, the nature of the slurry could be profoundly different from that being attempted for Phase I testing, an alternative assumption that is beyond the stated scope of this report.

The significance of the short characteristic time constant for diffusion-limited leaching obtains independence for the actual leach rate from mass transfer for the following reason. If the leach duration is long compared to the diffusion-limited time constant, then mass-transfer effects are unimportant and hence mixing at the scale of a particle is unimportant. If the leach duration is comparable to or shorter than the diffusion-limited time constant, then the effect of mass transfer does affect the leach rate, but determining the leach rate is not important to meeting WTP objectives because the conversion would occur far too fast for the rate to be measured - in the PEP or the WTP, the conversion would appear to be instantaneous. Therefore, in any event, the magnitude of the diffusion-limited time constant signifies that mass-transfer effects on leaching cannot be important to interpreting leach data from the PEP.

\section{A.2.2.4 Effect of Stochastic Non-Uniformity}

There are two important sources of uncertainty in predicting leach factors: knowing the true values of the parameters, and knowing the spatial uniformity of the parameters. The first uncertainty results from measurement uncertainty: inferring the true value through a mass balance based on imperfect measurements. Probably the most important measurement error will be of temperature because the effect of temperature is great. The measurement error also depends on uniformity, as discussed further below.

The second uncertainty results from imperfect physical uniformity: having to assume spatial uniformity in the true values when actually there are variations. Both kinds of uncertainty cause an error in predicting the conversion and hence in predicting the leach factor. Here we consider the effect of spatial uniformity.

The associated error may or may not be important compared to the measurement error; that cannot be determined except from analyzing physical samples obtained over multiple locations in tanks in the PEP. The following analysis of the effect of spatial uniformity will help determine the significance of the spatial variability we are trying to measure through sampling.

The mass of remaining reacted solids at time $t$ in some differential mass fraction $d m(0)$ of the initially unreacted solids is

$$
d m(t)=\left(1-X\left(t, \xi_{i}\right)\right) d m(0)
$$

where the set $\xi_{\mathrm{i}}$ is the parameters $\mathrm{T}, \mathrm{C}_{\mathrm{OH}-}, \mathrm{C}_{\mathrm{AlO} 2-}, \varphi_{\mathrm{S} 0}$ on which the conversion depends, evaluated in the mass element. Integrating over the total mass,

$$
m(t)=\int_{m_{\text {total }}}\left(1-X\left(t, \xi_{i}\right)\right) d m(0)=m(0)-\int_{m_{\text {total }}} X\left(t, \xi_{i}\right) d m(0)
$$


Assume that over the volume elements there is some distribution of these parameters about their values averaged over the total slurry volume. We can approximate this using a Taylor series as

$$
X\left(t, \xi_{i}\right)=X\left(t, \bar{\xi}_{i}\right)+\sum_{i}\left(\frac{d X}{d \xi_{i}}\right)_{\bar{\xi}_{i}} \delta \xi_{i}+\frac{1}{2} \sum_{i}\left(\frac{d^{2} X}{d \xi_{i}^{2}}\right)_{\bar{\xi}_{i}}\left(\delta \xi_{i}\right)^{2}+\cdots
$$

where we have assumed no correlation between the variations of parameters about their means.

Substituting,

$$
m(t)=m(0)-\int_{m(0)}\left(X\left(t, \bar{\xi}_{i}\right)+\sum_{i}\left(\frac{d X}{d \xi_{i}}\right)_{\bar{\xi}_{i}} \delta \xi_{i}+\frac{1}{2} \sum_{i}\left(\frac{d^{2} X}{d \xi_{i}^{2}}\right)_{\bar{\xi}_{i}}\left(\delta \xi_{i}\right)^{2}+\cdots\right) d m(0)
$$

Rearranging,

$$
m(t)=m(0)-X\left(t, \bar{\xi}_{i}\right) \int_{m(0)} d m(0)-\sum_{i}\left(\frac{d X}{d \xi_{i}}\right) \int_{\bar{\xi}_{i}} \int_{m(0)} \delta \xi_{i} d m(0)-\frac{1}{2} \sum_{i}\left(\frac{d^{2} X}{d \xi_{i}^{2}}\right) \int_{\bar{\xi}_{i}} \int_{m(0)}\left(\delta \xi_{i}\right)^{2} d m(0)+\cdots
$$

The first integral is the initial mass, and by the definition of the variation in the parameters, the second integral is zero. Then

$$
m(t)=m(0)-X\left(t, \bar{\xi}_{i}\right) m(0)-\frac{1}{2} \sum_{i}\left(\frac{d^{2} X}{d \xi_{i}^{2}}\right)_{\bar{\xi}_{i}} \int_{m(0)}\left(\delta \xi_{i}\right)^{2} d m(0)+\cdots
$$

The average conversion is

$$
\bar{X}=\frac{m(0)-m}{m(0)}=X\left(t, \bar{\xi}_{i}\right)+\frac{1}{2} \sum_{i} \Theta_{i} \int_{m(0)}\left(\delta \xi_{i}\right)^{2} \frac{d m(0)}{m(0)}+\cdots \quad \Theta_{i}=\left(\frac{d^{2} X}{d \xi_{i}^{2}}\right)_{\bar{\xi}_{i}}
$$

Rearranging, the departure of the average conversion from the conversion that would occur if the parameters occurred uniformly at their average values is

$$
\delta X=\bar{X}-X\left(t, \bar{\xi}_{i}\right)=\frac{1}{2} \sum_{i} \Theta_{i} \int_{m(0)}\left(\delta \xi_{i}\right)^{2} \frac{d m(0)}{m(0)}
$$

The integrals over the second variations $\left(\delta \xi_{i}\right)^{2}$ are inherently positive. Hence, the effect of the variations on the average conversion depends on both the magnitude of the variations and the sign of the second derivatives $\Theta_{i}$ of the conversion $X$ relative to the parameters $\xi_{i}$.

We assume that:

- a sufficiently uniform concentration of the reagent can be established before significant leaching occurs and that its second variation is small 
- sufficient reagent is added that its concentration is not affected much (e.g., probably $<10 \%$ ) by the conversion

- the Gibbsite dissolves fast enough that there will be time for the resulting $\mathrm{Al}(\mathrm{OH})_{4}{ }^{-}$to be distributed sufficiently uniformly that also its second variation is small.

The remaining key variations are of temperature and the initial solids volume fraction. The temperature might remain non-uniform due to imperfect mixing while the heat lost due to sparging and at the sides of the tank is replaced by steam injection. The solids volume fraction might remain non-uniform if some of the solids settle or they are held in a non-uniform vertical distribution by continuous competition between upward pulses and settling.

The effect of temperature on chemical reactions is usually important. It is often described in terms of the Ahrrenius representation of the reaction rate constant. That is,

$$
r \propto e^{-(E / R T)} \quad \frac{d r}{d T}=r \frac{(E / R T)}{T} \quad \frac{d^{2} r}{d r^{2}}=r \frac{(E / R T)^{2}}{T^{2}}-r \frac{2(E / R T)}{T^{2}}=r \frac{(E / R T)}{T^{2}}((E / R T)-2)
$$

and hence, $\Theta_{T}$ is positive for $(E / R T)>2$. The caustic leaching occurs at roughly $100^{\circ} \mathrm{C}$; then $\Theta_{T}>0$ for $E>6.2 \mathrm{~kJ} / \mathrm{mol}$, which is in fact found for the temperature dependence of leaching the Boehmite. Therefore, $\Theta_{T}$ is positive.

The development for $\Theta_{\varphi}$ is more involved, as follows.

$$
\begin{gathered}
\left(\frac{\partial}{\partial \phi_{S 0}}\right)_{t, T}\left[\int_{0}^{X} \frac{d X}{f\left(X, \phi_{S 0}\right)}-\frac{\rho_{S} A_{S}(0) r(0)}{m_{s, 0}} t\right]=0 \quad f\left(X, \phi_{S 0}\right)=\widetilde{A}(X) \widetilde{r}\left(X, \phi_{S 0}\right) \\
\left(\frac{\partial X}{\partial \phi_{S 0}}\right)_{t, T} \frac{1}{f\left(X, \phi_{S 0}\right)}-\int_{0}^{X}\left(\frac{\partial f\left(X, \phi_{S 0}\right)}{\partial \phi_{S 0}}\right)_{t, T} \frac{d X}{f\left(X, \phi_{S 0}\right)^{2}}=0 \\
\tau=\int_{0}^{X} \frac{1}{f} d X \quad f=\widetilde{r}\left(X, \phi_{S 0}\right) \widetilde{A}(X)
\end{gathered}
$$

Differentiate with respect to $\varphi_{\mathrm{S} 0}$ at constant $\tau$ to get.

$$
0=\frac{X_{\phi}}{f}+\int_{0}^{X}\left(\frac{1}{f}\right)_{\phi} d X \quad X_{\phi} \equiv\left(\frac{\partial X}{\partial \phi_{S 0}}\right)_{\tau} \quad\left(\frac{1}{f}\right)_{\phi} \equiv\left(\frac{\partial}{\partial \phi_{S 0}} \frac{1}{f}\right)_{\tau}
$$

Differentiate again to get

$$
0=\frac{X_{\phi \phi}}{f}-\frac{X_{\phi} f_{\phi}}{f^{2}}+X_{\phi}\left(\frac{1}{f}\right)_{\phi}+\int_{0}^{X}\left(\frac{1}{f}\right)_{\phi \phi} d X
$$


Solve for $X_{\varphi}$ :

$$
X_{\phi}=-f \int_{0}^{X}\left(\frac{1}{f}\right)_{\phi} d X
$$

Solve for $X_{\varphi \varphi}$ :

$$
X_{\phi \phi}=\frac{X_{\phi} f_{\phi}}{f}-X_{\phi} f\left(\frac{1}{f}\right)_{\phi}-f \int_{0}^{X}\left(\frac{1}{f}\right)_{\phi \phi} d X=X_{\phi}\left(2 \frac{f_{\phi}}{f}\right)-f \int_{0}^{X}\left(\frac{1}{f}\right)_{\phi \phi} d X
$$

Combine:

$$
X_{\phi \phi}=-2 f_{\phi} \int_{0}^{X}\left(\frac{1}{f}\right)_{\phi} d X-f \int_{0}^{X}\left(\frac{1}{f}\right)_{\phi \phi} d X=2 f^{2}\left(\frac{1}{f}\right)_{\phi} \int_{0}^{X}\left(\frac{1}{f}\right)_{\phi} d X-f \int_{0}^{X}\left(\frac{1}{f}\right)_{\phi \phi} d X
$$

Expand the derivatives

$$
\left(\frac{1}{f}\right)_{\phi}=-\frac{f_{\phi}}{f^{2}} \quad\left(\frac{1}{f}\right)_{\phi \phi}=-\frac{f_{\phi \phi}}{f^{2}}+\frac{2 f_{\phi}^{2}}{f^{3}}
$$

Substitute:

$$
X_{\phi \phi}=2 f_{\phi} \int_{0}^{X} \frac{f_{\phi}}{f^{2}} d X-f \int_{0}^{X} \frac{2 f_{\phi}^{2}-f f_{\phi \phi}}{f^{3}} d X
$$

Expand the derivatives again

$$
f_{\phi}=\frac{\partial f}{\partial \phi_{S 0}}=\frac{\partial}{\partial \phi_{S 0}}\left(\widetilde{r}\left(X, \phi_{S 0}\right) \widetilde{A}(X)\right)=\widetilde{A}(X) \frac{\partial \widetilde{r}\left(X, \phi_{S 0}\right)}{\partial \phi_{S 0}}
$$

Expand again:

$$
\frac{\partial \widetilde{r}\left(X, \phi_{S 0}\right)}{\partial \phi_{S 0}}=\frac{1}{r(0)} \frac{\partial r\left(X, \phi_{S 0}\right)}{\partial \phi_{S 0}}=\frac{r_{0}}{r(0)} \frac{\partial}{\partial \phi_{S 0}}\left(1-\left(\frac{C_{P}}{C_{P, e q}}\right)^{2}\right)=-\frac{r_{0}}{r(0)} \frac{2}{C_{P, e q}}\left(\frac{C_{P}}{C_{P, e q}}\right)\left(\frac{\partial C_{P}}{\partial \phi_{S 0}}\right)
$$

Differentiate again:

$$
\frac{\partial^{2} \widetilde{r}\left(X, \phi_{S 0}\right)}{\partial \phi_{S 0}{ }^{2}}=-\frac{r_{0}}{r(0)} \frac{2}{C_{P, e q}{ }^{2}}\left(\frac{\partial C_{P}}{\partial \phi_{S 0}}\right)^{2}-\frac{r_{0}}{r(0)} \frac{2}{C_{P, e q}}\left(\frac{C_{P}}{C_{P, e q}}\right)\left(\frac{\partial^{2} C_{P}}{\partial \phi_{S 0}{ }^{2}}\right)
$$

For Boehmite, the function $f_{P}$ is $\left(C_{P} / C_{p, e q}\right)^{2}$ where $C_{P, e q}$ is the equilibrium concentration of reaction product for the reversible reaction, which is a function of both reagent concentration and temperature. 
For a given temperature and assuming the initial reagent concentration is great enough that the reagent concentration is approximately constant over the course of the reaction, then approximately $C_{P, e q}$ is constant. Then

$$
\begin{gathered}
\frac{\partial r}{\partial \phi_{S 0}}=\frac{\partial r}{\partial C_{p}} \frac{\partial C_{P}}{\partial \phi_{S 0}} \\
\frac{\partial C_{P}}{\partial \phi_{S 0}}=v_{P} \rho_{S} X \quad \frac{\partial^{2} C_{P}}{\partial \phi_{S 0}{ }^{2}}=0
\end{gathered}
$$

Substitute

$$
\frac{\partial \widetilde{r}\left(X, \phi_{S 0}\right)}{\partial \phi_{S 0}}=-\frac{r_{0}}{r(0)} \frac{2 C_{P}}{C_{P, e q}^{2}} v_{P} \rho_{S} X
$$

Differentiate again

$$
\frac{\partial^{2} \widetilde{r}\left(X, \phi_{S 0}\right)}{\partial \phi_{S 0}^{2}}=-\frac{r_{0}}{r(0)} \frac{2}{C_{P, e q}^{2}}\left(\frac{\partial C_{P}}{\partial \phi_{S 0}}\right)^{2}
$$

Substitute

$$
f_{\phi}=-2 \frac{r_{0} \tilde{A}(X) v_{P} X}{r(0)}\left(\frac{\rho_{S}}{C_{P, e q}}\right)\left(\frac{C_{P}}{C_{P, e q}}\right)
$$

Differentiate again:

$$
f_{\phi \phi}=-2 \frac{r_{0} \tilde{A}(X)}{r(0)} \frac{v_{P} \rho_{S} X}{C_{P, e q}^{2}}\left(\frac{\partial C_{P}}{\partial \phi_{S 0}}\right)=-2 \frac{r_{0} \tilde{A}(X)\left(v_{P} X\right)^{2}}{r(0)}\left(\frac{\rho_{S}}{C_{P, e q}}\right)^{2}
$$

Therefore

$$
2 f_{\phi}^{2}-f f_{\phi \phi}=\left[2+6\left(\frac{C_{P}}{C_{P, e q}}\right)^{2}\right]\left(\frac{r_{0}}{r(0)}\right)^{2}\left(v_{P} X\right)^{2} \tilde{A}(X)^{2}\left(\frac{\rho_{S}}{C_{P, e q}}\right)^{2}
$$

Thus, the signs of the terms forming $X_{\varphi \varphi}$ are

$$
X_{\phi \phi}=2 f_{\phi} \int_{0}^{X} \frac{f_{\phi}}{f^{2}} d X-f \int_{0}^{X} \frac{2 f_{\phi}^{2}-f f_{\phi \phi}}{f^{3}} d X=2(<0) \int_{0}^{X} \frac{f_{\phi}}{f^{2}} d X-f \int_{0}^{X} \frac{(>0)}{f^{3}} d X
$$


Therefore,

$$
\Theta_{\phi}=X_{\phi \phi}<0
$$

In summary, $\Theta_{T}$ is positive, and $\Theta_{\varphi}$ is negative. Therefore, for a given average temperature, the effect of a non-uniform temperature is to cause a conversion that is greater than for a uniform temperature. Conversely, for a given average initial volume fraction of reactive solids, the effect of a non-uniform initial distribution of the volume fraction is to cause a conversion that is lesser than for a uniform solids volume fraction. That is, these are corrections that must be made to the conversion predicted based on the average temperature and average solids volume fractions, which are subject to measurement error.

\section{A.2.2.5 Similitude in Chemical Conversion}

During operation of the PTF, the average solids volume fraction will be calculated from mass balances. We presume there will be no correlation between the error in this estimate and any spatial nonuniformity in the solids volume fraction. On the other hand, the temperature will be measured at a single location and inferred to be the average temperature. Clearly, if the temperature is significantly nonuniform, the single-point measurement can be in error both due to measurement error per se and because it happens to be taken at a location with a significant spatial variation from the true average temperature. Thus, we can expect that non-uniformity in the temperature can cause both measurement error and error in the mass balance calculation of the conversion, thus increasing the important of characterizing any nonuniformity of the temperature in the PEP and predicting from that the degree of uniformity expected in the PTF.

To establish similitude in the chemical conversion between the PEP and the PTF, we need to match at the start of the caustic leach process the

- volume fraction of the reactive solid

- particle size distribution of the reactive solid

- concentration of hydroxide

- concentration of "aluminate"

and create the same temperature-time sequence.

Then we establish similitude from which we would expect the conversion to develop with time in the PEP as in the PTF.

From the analysis above, we also require a uniform spatial distribution in either system of the parameters being matched between the systems. We cannot predict the required degree of uniformity until we examine and interpret PEP data, taking into account the significant uncertainty in the chemical reaction rate parameters determined in the laboratory.

\section{A.2.3 Filtration}

As discussed in Section 3.7, the filtration rate is affected both by the accumulation over time of fines in the filter media and the accumulation over time of any filter cake on the filter media. Because the PEP will include full-scale, prototype filters operated with the prototype trans-membrane pressure and 
prototype cross-flow velocity, the remaining issue is similitude in time related to the fine and coarse solids in the filter feed. These properties change with time through a mass balance over the filter feed tank and filter. The mass balance is affected by the uniformity of solids over the volume of the feed tank. Treating the uniformity separately, first assume a uniform concentration in the feed tank.

The following equations are based on the assumption that, for the purpose of the report, solids can be divided into a portion too fine to be rejected by the filter media, defined as "fines," and the rest, defined as "coarse." Then the fines follow the liquid into the filter media, while coarse solids either are stripped by the cross flow or overwhelm the cross flow and add to the filter cake. Then some can be stripped off the cake as well. The concentration of fines (as defined) is assumed to be too small to impact the filter cake compared to coarse solids.

The resulting mass balance over the tank and the filter of fine (subscript "--") solids is

$$
\frac{d\left(V_{S} C_{-}\right)}{d t}=-A_{F} u_{F} C_{-}=-A_{F} C_{F-} \frac{d \delta_{-}}{d t}
$$

where $V_{S}$ is the volume of slurry in the filter feed tank, $C_{\text {. }}$ is the mass concentration of fine solids in the slurry, $t$ is time, $A_{F}$ is the area of the filter media, $u_{F}$ is the filter flux expressed as the superficial velocity of filtrate through the filter media, $\delta_{\text {- }}$ is the thickness of fines accumulated in the filter media, and $C_{F-}$ is the superficial concentration of the fines caught in the filter media. The mass balance over the tank and filter of coarse (subscript "+") solids is

$$
\frac{d\left(V_{S} C_{+}\right)}{d t}=-\left(\varepsilon_{X} u_{F} C_{+}-C_{F+} \dot{V}_{X}\right) A_{F} H\left(\delta_{+}\right)=-A_{F} C_{F+} \frac{d \delta_{+}}{d t}
$$

where $C_{+}$is the mass concentration of coarse solids in the slurry, $C_{F^{+}}$is the concentration of coarse solids in filter cake, $\varepsilon_{X}$ is the fraction of solids approaching the filter that are caught by the filter cake (decreases with cross-flow velocity), $\dot{V}_{X}$ is the rate (volume/area/time) of removal of filter cake by the cross-flow (increases with cross-flow velocity), $\delta_{+}$is the thickness of the filter cake, and $H\left(\delta_{+}\right)$is zero if $\delta_{+}$is zero and unity if it is positive. Finally, a mass balance of the liquid phase of the slurry over the tank and filter is

$$
\frac{d\left(V_{S} C_{L}\right)}{d t}=-A_{F} u_{F} C_{L}
$$

where $\mathrm{C}_{\mathrm{L}}$ is the mass concentration of the liquid phase. The filter flux is diminished both by accumulation of fines in the filter media and accumulation of filter cake on the media. Therefore,

$$
u_{F}=u_{F}\left(\delta_{-}, \delta_{+}\right)
$$


Define the following dimensionless variables.

$$
\begin{gathered}
\widetilde{V}_{S} \equiv V_{S}(t) / V_{S 0} \quad V_{S 0} \equiv V_{S}(t=0) \\
\widetilde{u}_{F} \equiv u_{F}(t) / u_{F 0} \quad u_{F 0} \equiv u_{F}\left(\delta_{-}=0, \delta_{+}=0\right) \\
\tau \equiv A_{F} u_{F 0} t / V_{S 0}
\end{gathered}
$$

Substituting into the mass balances,

$$
\begin{gathered}
\frac{d\left(\widetilde{V}_{S} C_{-}\right)}{d \tau}=-\widetilde{u}_{F}\left(\delta_{-}, \delta_{+}\right) C_{-}=-\left(\frac{A_{F} C_{F-}}{V_{S 0}}\right) \frac{d \delta_{-}}{d \tau} \\
\frac{d\left(V_{S} C_{+}\right)}{d \tau}=-\left(\varepsilon_{X} \widetilde{u}_{F}\left(\delta_{-}, \delta_{+}\right) C_{+}-\frac{C_{F+} \dot{V}_{X}}{u_{F 0}}\right) H\left(\delta_{+}\right)=-\left(\frac{A_{F} C_{F+}}{V_{S 0}}\right) \frac{d \delta_{+}}{d \tau} \\
\frac{d\left(\widetilde{V}_{S} C_{L}\right)}{d \tau}=-\widetilde{u}_{F}\left(\delta_{-}, \delta_{+}\right) C_{L}
\end{gathered}
$$

These comprise five transient mass balances (differential equations) in the five dependent functions of $\tau, \mathrm{C}_{-}, \mathrm{C}_{+}, \mathrm{C}_{\mathrm{L}}, \delta_{\text {-, and }} \delta_{+}$. Initially $\delta_{-}=0$ and $\delta_{+}=0$ and the initial concentrations are known. A unique solution to the equations follows from these initial conditions. The equations include the parameters $\left(A_{L} / V_{S 0}\right) C_{F-},\left(A_{L} / V_{S 0}\right) C_{F+}$, and $\varepsilon_{X}$. Therefore, these equations have solutions for the concentrations of the form $C_{-,+, L}=f\left(\tau ;\left(A_{L} / V_{S 0}\right) C_{F-,}\left(A_{L} / V_{S 0}\right) C_{F+}, \varepsilon_{X}\right)$ and for the depths of the form

$$
\delta_{-,+}=f\left(\tau ;\left(A_{L} / V_{S 0}\right) C_{-,}\left(A_{L} / V_{S 0}\right) C_{F+}, \varepsilon_{X}\right)
$$

Therefore, the functional dependence of the filter flux is

$$
\tilde{u}_{F}=f\left(\tau ;\left(A_{F} / V_{S 0}\right) C_{-},\left(A_{F} / V_{S 0}\right) C_{+}, \varepsilon_{X}\right)
$$

The solids being filtered determine the values $C_{F-}, C_{F+}$, and $\varepsilon_{X}$. Therefore, for the same solids, by matching the ratio of filter area to initial solids volume $A_{F} / V_{S O}$ between the PEP and the PTF we should find the same change in the filtration rate if expressed in terms of the dimensionless time

$$
\tau=\left(A_{F} u_{F 0} / V_{S 0}\right) t
$$

And, given the same solids, the ratio of filter area to solids volume is matched by matching the ratio of filter area to initial solids mass - the two specifications are interchangeable.

Thus, dimensionless transient mass balances describing the key phenomena affecting the filtration rate show similitude is established by the following.

- Between the PEP and the PTF, match the properties of the solids being filtered

- Between the PEP and the PTF, match the ratio of filter area to initial mass of solids being filtered 
The resulting filter flux compared to that for a clean filter measured in the PEP will decrease as the same function of dimensionless time as in the PTF. By using the same filter media and matching the filter area to the initial solids mass, then the ratio of dimensionless times will be the ratio of actual time. Then the resulting filter flux compared to that for a clean filter measured in the PEP will decrease as the same function of time as in the PTF.

If similitude is established as described above, the filter flux measured in the PEP is a prediction of that expected in the PTF at the same times elapsed after establishing a clean filter.

\section{A.3 Non-Uniform Concentrations in Tanks}

Because the wash and leach factors are predicted from mass balances combined with laboratory measurements and process measurements, and because the mass balances are predicated on a uniform concentration and temperature in tanks, the principal concerns about predicting the wash and leach factors are about the uniformity of concentrations and temperatures in the main UFP tanks. The concern about predicting the filtration rate is due to concerns about ability to predict performance full scale from measurements at a smaller scale because of concerns about adequate understanding of the interrelated phenomena controlling the filtration rate.

Mixing in the slurry and settling of solids from the slurry, mixed or not, potentially affect the uniformity of characteristics that control the washing process, leaching processes, and the filter feed. Mixing is induced primarily by PJM, often augmented by the circulation through the filter loop and the tank connected to it. Mixing consists of turbulent transport throughout the volume of the slurry of momentum injected at the pulse jet nozzles, the circulation return nozzle, or by rising bubbles from an air sparger. Dispersion is impeded by viscous dissipation of momentum to heat occurring mainly in small eddies in turbulent flow.

Because pulse jet mixing is crucial to many operations in the PTF, the EFRT singled out PJM operation as worthy of a separate study; this issue is denoted M3. The WTP developed an issue response plan for M3 in which pulse jet mixing experiments in both Newtonian and non-Newtonian slurries are designed based on principles of similitude and being characterized and correlated. These results are being obtained in parallel with the writing of this report. Eventually, this report can be revised to use engineering correlations developed by the WTP to replace the approximate relationships provided herein based on dimensional analysis of experiments that are related to PTF processes but were not established for the sake of similitude.

Below we consider two conceptual departures from ideal mixing: settling of solids and unmixed fluid regions. The first concept is a result of mixing being locally insufficient to overcome particle settling. The second concept is a result of mixing being locally insufficient to overcome phenomena impeding fluid motion such as viscous stress or a yield stress.

\section{A.3.1 Combined Settling and Turbulent Dispersion}

Newtonian slurry being agitated by PJM is beyond straightforward computation. The best we can do is to interpret related experimental studies using principles of dimensional analysis to relate the studies to the PTF, as follows. 


\section{A.3.1.1 Particle Settling}

Begin with the physics of settling for a single particle. As a particle moves through the fluid in which it is embedded, it experiences a force related to the velocity of the particle relative to the fluid. The relationship can be described as a dimensionless "drag coefficient" $C_{D}$, which is defined by

$$
F=A C_{D}\left(\frac{1}{2} \rho_{L} u_{S}^{2}\right)
$$

where $F$ is the magnitude of the force caused by the relative velocity $u_{S}$, and $A$ is the cross-sectional area of the particle in a plane perpendicular to the relative velocity. Dimensional analysis shows that $C_{D}$ is a function of the Reynolds number based on the relative velocity and the particle diameter, $R e_{p}$. For sufficiently small $R e_{p}$, the $C_{D}$ is inversely proportional to $R e_{p}$; for sufficiently large $R e_{p}, C_{D}$ is independent of $R e_{p}$. The gravitational force acting on the particle is

$$
F=\left(\rho_{S}-\rho_{L}\right) V g
$$

where $V$ is the volume of the particle. At the settling velocity of the particle these forces balance; hence

$$
u_{S}^{2}=\frac{1}{C_{D}\left(R e_{p}\right)}\left(\frac{\rho_{S}-\rho_{L}}{\rho_{L}}\right)\left(\frac{2 V}{A}\right) g
$$

For a sphere, $2 V / A=D_{p} / 3$. Then,

$$
\operatorname{Re}_{p}^{2}=\left(\frac{u_{S} D_{p}}{v}\right)^{2} \propto\left(\frac{D_{p}}{v}\right)^{2} \frac{1}{C_{D}\left(\operatorname{Re}_{p}\right)}\left(\frac{\rho_{S}-\rho_{L}}{\rho_{L}}\right) D_{p} g
$$

Rearranging, we define a function $F_{R e}\left(R_{p}\right)$ as

$$
F_{\mathrm{Re}}\left(\operatorname{Re}_{p}\right)=\operatorname{Re}_{p}^{2} C_{D}\left(\operatorname{Re}_{p}\right) \propto \frac{D_{p}^{3} g}{v^{2}}\left(\frac{\rho_{S}-\rho_{L}}{\rho_{L}}\right)
$$

Inverting,

$$
\operatorname{Re}=F_{\operatorname{Re}}^{-1}\left(\frac{D_{p}^{3} g}{v^{2}}\left(\frac{\rho_{S}-\rho_{L}}{\rho_{L}}\right)\right)
$$

Then the settling velocity is

$$
u_{S}=\frac{v}{D_{p}} \operatorname{Re}=\frac{v}{D_{p}} F_{\operatorname{Re}}^{-1}\left(\frac{D_{p}^{3} g}{v^{2}}\left(\frac{\rho_{S}-\rho_{L}}{\rho_{L}}\right)\right)=f\left(D_{p},\left(D_{p}^{3} g\left(\rho_{S}-\rho_{L}\right) / \rho_{L} v^{2}\right), v\right)
$$

For sufficiently small particles, the settling velocity is as is discussed in Section A.4.3.3. 


\section{A.3.1.2 Dispersion}

The settling velocity is downward and relative to the fluid motion. Hence, relative to the system containing the fluid, the velocity is

$$
\mathbf{u}_{p}=\mathbf{u}+u_{S} \mathbf{e}_{g}
$$

where $\mathbf{u}_{p}$ is the particle local velocity, $\mathbf{u}$ is the fluid local velocity, and $\mathbf{e}_{g}$ is direction of the acceleration of gravity. The mass flux of particles averaged over some horizontal plane is

$$
\left\langle\rho_{S} \phi \mathbf{u}_{p}\right\rangle=\rho_{S}\left\langle\phi \mathbf{u}+\phi u_{S} \mathbf{e}_{g}\right\rangle \doteq \rho_{S}\langle\phi \mathbf{u}\rangle+\rho_{S}\langle\phi\rangle u_{S} \mathbf{e}_{g}
$$

where $\varphi$ is the volume fraction of the particles and \langle\rangle signifies averaging over time and horizontal area. For turbulent flow, the direction and magnitude of the fluid velocity $\mathbf{u}$ is nearly random in time and position; hence the component of the mass flux due to the fluid motion can be treated as a diffusive phenomenon characterized by a dispersion coefficient $D_{u}$ :

$$
\left\langle\rho_{S} \phi \mathbf{u}_{p}\right\rangle \doteq \rho_{S} D_{u} \frac{d\langle\phi\rangle}{d z}+\rho_{S}\langle\phi\rangle u_{S} \mathbf{e}_{g}
$$

Assuming the vertical distribution of particles attains a steady state, then the mass flux is zero at every vertical location; that is,

$$
0 \doteq \rho_{S}\left(-D_{u} \frac{d\langle\phi\rangle}{d z}\right) \mathbf{e}_{g}+\rho_{S}\langle\phi\rangle u_{S} \mathbf{e}_{g}
$$

Rearranging,

$$
\frac{d \ln \langle\phi\rangle}{d z}=L_{0} \frac{d \ln \langle\phi\rangle}{d z}=\frac{u_{S} L_{0}}{D_{u}}
$$

Thus, scaled to the size of the system, the degree of non-uniformity of the solids volume fraction over the height of the fluid depends on the dimensionless group $u_{S} L_{0} / D_{u}$, which has the form of a Reynolds number.

\section{A.3.1.3 Dispersion Coefficient}

It remains to determine how $D_{u}$ depends on the size and characteristic velocity in a system. For fully turbulent mixing, we can assume mixing does not depend significantly on viscosity. Likely independent parameters are the same set considered in the previous section on suspension of solids by a submerged jet: $L_{0}, u_{0}, \rho_{L}, \rho_{S}, C_{w}, D_{p}$, and geometric ratios in the tank and contents. 
From dimensional analysis,

$$
D_{u} / u_{0} L_{0} \propto g_{D}\left(\left(\rho_{S}-\rho_{L}\right) / \rho_{L}, C_{w}, D_{p} / L_{0}\right)
$$

where $g_{D}$ is a dimensionless function of material properties, and hence, for a given material, we can treat the function as a constant: $g_{D}$. Then

$$
u_{S} L_{0} / D_{u} \propto u_{S} / u_{0} g_{D}
$$

One obtains the same result by starting with a dimensional analysis of the system parameters if we include $u_{S}$ directly as a parameter, e.g., in place of $D_{p}$.

Alternatively, one could attempt a more mechanistic estimate of $\mathrm{D}_{\mathrm{u}}$ based on

$$
D_{u} \sim L_{m i x}^{2} / t_{m i x}
$$

where $L_{m i x}$ and $t_{m i x}$ are local characteristic mixing distances and mixing times. If we envision the mixing to result from disruption and dissipation of a submerged jet as discussed in Section A.4.3.5, we have

$$
t_{\text {mix }} \sim D(z) / u(z)
$$

where $\mathrm{D}(\mathrm{z})$ is the nominal jet diameter and $\mathrm{u}(\mathrm{z})$ is the nominal jet velocity, each a function of distance along the jet from its origin. Taking $L_{m i x}=D(z)$, then

$$
D_{u} \sim D(z)^{2} /(D(z) / u(z))=D(z) u(z)
$$

For a submerged jet, we have

$$
J_{0}=A_{0} \rho u_{0}^{2}=\rho \frac{\pi}{4} D(z)^{2} u(z)^{2}
$$

where $J_{0}$ is the momentum flow in the jet at its origin. Then

$$
D_{u} \sim \sqrt{4 J_{0} / \pi \rho}
$$

That is, for the underlying assumption that the jet momentum flow is conserved, which at best is true only if the fluid is Newtonian, we expect the dispersion coefficient not to vary substantially along the length of a jet. Since

$$
J_{0} \propto u_{0}^{2} L_{0}^{2}
$$

again we find

$$
D_{u} \propto u_{0} L_{0}
$$

again corresponding to

$$
u_{S} L_{0} / D_{u} \propto u_{S} / u_{0} g_{D}
$$


Then we have

$$
\frac{d \ln \langle\phi\rangle}{d \widetilde{z}}=L_{0} \frac{d \ln \langle\phi\rangle}{d z}=\frac{u_{S} L_{0}}{D_{u}}
$$

However, the actual situation is that multiple jets originate downward arrayed circumferentially near the floor of the tank, are turned upward by the tank walls, and proceed upward and bounded by the static tank wall and the pulse jet tanks, thereby dissipating the jet momentum flow by shear forces at the static boundaries. Hence, an alternative approach is to recognize that actually the momentum flow $J$ decreases with distance along the upward course of the jets; a plausible dimensional approach is to assume

$$
J(z)=J_{0}(1-(z / h))
$$

where $h$ is the height of the mixed region (see Section A.4.3.5 about potential constraints on $h$ ). Then

$$
J_{0}(1-(z / h))=\rho A(z) u(z)^{2}
$$

Because the flow cross-section is constrained by the total horizontal area, then in that region

$$
A(z) \propto L_{0}^{2}
$$

Combining,

$$
J(z) \propto A_{0} \rho u_{0}^{2}(1-(z / h)) \propto \rho L_{0}^{2} u(z)^{2}
$$

and hence

$$
u(z) \propto u_{0} \sqrt{1-(z / h)}
$$

At the same time, the characteristic dispersion length is constrained to some multiple (given geometric similarity) of $L_{0}$ instead of the jet diameter that would obtain for a transversely unconstrained jet. Then the characteristic mixing time is

$$
t_{\text {mix }} \propto L_{0} / u_{0} \sqrt{1-(z / h)}
$$

and hence the dispersion coefficient is

$$
D_{u} \propto L_{0}^{2} /\left(L_{0} / u_{0} \sqrt{1-(z / h)}\right)=L_{0} u_{0} \sqrt{1-(z / h)}
$$

Returning to the vertical mass balance,

$$
\frac{d \ln \langle\phi\rangle}{d \widetilde{z}}=L_{0} \frac{d \ln \langle\phi\rangle}{d z} \propto \frac{u_{S}}{u_{0}} \sqrt{1-(z / h)}=\frac{u_{S}}{u_{0}} \frac{1}{\sqrt{1-\widetilde{z} /\left(h / L_{0}\right)}}
$$

If, as is suggested by the analysis of Section A.4.3.5, similitude can be established such that the ratio $h / L_{0}$ is preserved between systems. Assuming this, either because $\mathrm{h}$ is set directly by process control or 
by creating similar mixing caverns, if caverns exist, then again the uniformity of the average solids volume fraction depends on the ratio $u_{S} u_{0}$, which for a given material (given $u_{S}$ ), depends on $u_{0}$.

\section{A.3.1.4 Significance}

From this analysis, we conclude that the solids distribution over the height of the turbulent fluid is uniform for sufficiently small values of $u_{S} / u_{0}$, which is intuitive. Further, we conclude that two systems will have the same degree of uniformity of the solids volume fraction - in the sense of the same shape of the volume fraction profile - if we match the ratio of settling velocity to the imposed characteristic velocity. We have shown above that, intuitively, the settling velocity depends only on the particle and fluid properties. Therefore, for two systems containing the same materials, the degree of uniformity in the solids volume fraction will be matched if the characteristic imposed velocity $u_{0}$ is matched.

However, the analysis includes the steady state condition that there is no mass flux at any vertical location and hence none at the flow of the tank, and hence, inherently that there is no accumulation of solids on the floor. Therefore, this analysis applies only to the question of uniformity above the tank floor, but not settling. The conditions required to avert settling are described in the example above of dimensional analysis and the correlation for the solids mass fraction that can be suspended without settling by a submerged jet mixing a slurry in a tank.

\section{A.3.2 Suspension of Settled Particles}

\section{A.3.2.1 Dimensional Analysis of Settling}

This analysis is a specific application of the general principles of dimensional analysis presented in Section A.1.5. Consider the example of a submerged jet suspending solid particles in nominally a Newtonian fluid but where interactions among particles causes an apparent stress-strain relationship that is not linear in the rate of strain tensor; that is, the fluid can be non-Newtonian but the form or descriptive parameters for the resulting stress-strain relationship are not known. The experimental parameters now include the density of both the liquid and solid (particle) phases, the viscosity of the pure liquid, and the acceleration of gravity.

Note that we circumvented this last parameter in the analysis of the conservation of momentum by reducing the pressure gradient to that of the pressure relative to the hydrostatic pressure. This is acceptable if none of the boundary conditions are in terms of pressure, such as occurs at a boundary between phases of differing density, as we now have due to the particles. Therefore, we must include $g$ as a parameter.

Further, we have more than one velocity to consider. The velocity of the fluid remains as a dependent variable, but each particle has a velocity. Considering particle individually is intractable, but we can define a volume-averaged velocity of particles relative to the volume-averaged fluid velocity, and, of course, for turbulent flow we include time in the averaging.

Further, because the distribution of the particles is not necessarily uniform, we have either the volume- and time-averaged solids volume fraction or particles or the volume- and time-averaged bulk density of the fluid and particles combined as a dependent variable. 
Combining, we have

$$
\begin{gathered}
\mathbf{u}=\mathbf{f}_{u}\left(\mathbf{x}, t, \mu, \rho_{L}, \rho_{S}, D_{p}, u_{0}, L_{0}, g, m_{S}\right) \\
\mathbf{u}_{p}=\mathbf{f}_{p}\left(\mathbf{x}, t, \mu, \rho_{L}, \rho_{S}, D_{p} u_{0}, L_{0}, g, m_{S}\right) \\
C_{w}=f_{w}\left(\mathbf{x}, t, \mu, \rho_{L}, \rho_{S}, D_{p}, u_{0}, L_{0}, g, m_{S}\right)
\end{gathered}
$$

where $u_{p}$ is the local average velocity of particles relative to the fluid, $C_{w}$ is the local average mass fraction of particles in the fluid, $\rho_{S}$ is the density of the particles, $D_{p}$ is the diameter of the particles, $m_{S}$ is the mass of solids in the system, and $f_{u}, f_{p}$, and $f_{w}$ are dimensional functions (vectors, for the velocities) of the arguments shown.

Applying dimensional analysis as discussed in Section A.1.5, we obtain

$$
\mathbf{u}=u_{0} \mathbf{g}_{u}\left(\widetilde{\mathbf{x}}, \widetilde{t}, \operatorname{Re},\left(\rho_{S} / \rho_{L}\right),\left(D_{p} / L_{0}\right),\left(u_{0}^{2} / g L_{0}\right),\left(m_{S} / \rho_{L} L_{0}^{3}\right)\right)
$$

where $\mathbf{g}_{u}$ is the dimensionless vector function corresponding to $\mathbf{f}_{u}$. The effect of the particles on the stress-strain relationship will depend on the volume fraction of particles, which depends on the mass fraction though the particle density. Hence, we need to retain the dimensionless group $\rho_{L} /\left(\rho_{S}-\rho_{L}\right)$ as a separate parameter. Considering the conservation of momentum separately for a single particle, we can further deduce that the form will be

$$
\mathbf{u}=u_{0} \mathbf{g}\left(\widetilde{\mathbf{x}}, \tilde{t}, \operatorname{Re},\left(\rho_{L} u_{0}^{2} /\left(\rho_{S}-\rho_{L}\right) g D_{p}\right),\left(m_{S} / \rho_{L} L_{0}^{3}\right)\right)
$$

where $D_{p}$ is the diameter of the particles. On the other hand, considering the effect of density differences over the full system, we expect the form to be

$$
\mathbf{u}=u_{0} \mathbf{g}\left(\widetilde{\mathbf{x}}, \widetilde{t}, \operatorname{Re},\left(\rho_{L} u_{0}^{2} /\left(\rho_{S}-\rho_{L}\right) g L_{0}\right),\left(m_{S} / \rho_{L} L_{0}^{3}\right)\right)
$$

Because both effects are potentially important, then we need to adopt the form

$$
\mathbf{u}=u_{0} \mathbf{g}\left(\widetilde{\mathbf{x}}, \widetilde{t}, \operatorname{Re},\left(\rho_{L} /\left(\rho_{S}-\rho_{L}\right)\right),\left(\rho_{L} u_{0}^{2} /\left(\rho_{S}-\rho_{L}\right) g L_{0}\right),\left(D_{p} / L_{0}\right),\left(m_{S} / \rho_{L} L_{0}^{3}\right)\right)
$$

where $\left(\rho_{L} u_{0}^{2} /\left(\rho_{S}-\rho_{L}\right) g L_{0}\right)$ is the densimetric Froude number, denoted Fr. The dimensionless group $\left(m_{S} / \rho_{L} L_{0}^{3}\right)$ is the characteristic mass fraction of particles for the system. This can be alternatively expressed in terms of the solids volume fraction or the characteristic mass fraction of solids in the liquid. If we choose the last of these and denote it as $C_{w 0}$, then we have

$$
\begin{gathered}
\widetilde{\mathbf{u}}=\mathbf{g}_{u}\left(\widetilde{\mathbf{x}}, \widetilde{t}, \operatorname{Re}, F r, \rho_{L} /\left(\rho_{S}-\rho_{L}\right), C_{w 0}\right) \\
\widetilde{\mathbf{u}}_{p}=\mathbf{u}_{p} / u_{0}=g_{p}\left(\widetilde{\mathbf{x}}, \widetilde{t}, \operatorname{Re}, \operatorname{Fr}, \rho_{L} /\left(\rho_{S}-\rho_{L}\right), C_{w 0}\right) \\
\widetilde{C}_{w}=C_{w} / C_{w 0}=g_{w}\left(\widetilde{\mathbf{x}}, \widetilde{t}, \operatorname{Re}, F r, \rho_{L} /\left(\rho_{S}-\rho_{L}\right), C_{w 0}\right)
\end{gathered}
$$


Assuming we identified all pertinent physical variables and parameters, we expect the above dimensionless expressions to describe the behavior of a given system of a given geometry. The length scale and time scale of a system are reflected in the parameters $\mathrm{L}_{0}$ and $\mathrm{L}_{0} / \mathrm{u}_{0}$, but to apply expressions such as these to systems of differing shape we must also characterize the shape though appropriate parameters. To do this comprehensively and generally is intractable, but for a given geometric class of systems only a few parameters are needed.

Consider the case of suspending solid particles in fluid in a circular tank of diameter $\mathrm{D}_{\mathrm{T}}$ with a deep, submerged jet with orifice of diameter $D_{J}$ and orifice velocity $u_{J}$. Including these in the dimensional analysis gives the same result except the dimensionless dependent variables are functions also of the dimensionless geometric parameters $D_{T} / L_{0}$ and $D_{J} / L_{0}$. However, we are free to choose $L_{0}=D_{T}$, reducing the number of additional parameters to one: $D_{J} / D_{T}$. The dimensionless material property parameter $D_{p} / L_{0}$ becomes $D_{p} / D_{T}$.

If our objective is to correlate the ability of a system to keep the solids suspended, we are not interested in the velocity distributions over the tank except as ultimately they determine the suspension of the solids, and are not interested in the variation of the solids mass fraction over the tank if we assume we are characterizing conditions where this is essentially uniform, and hence $\mathrm{C}_{\mathrm{w} 0}=\mathrm{C}_{\mathrm{w}, \max }$ where $\mathrm{C}_{\mathrm{w} \text {,max }}$ is the maximum solids mass fraction that can be suspended at the prescribed values of $R e, F r$, and the geometric and material parameters. Thus, our objective is information in the form

$$
\frac{C_{w, \max }}{C_{w 0}}=1=g_{w}\left(\operatorname{Re}, F r, D_{J} / D_{T}, D_{p} / D_{T}, C_{w, \max }\right)
$$

Since $C_{w}$ stands as a dimensionless argument, we can invert the dimensionless expression $g_{w}=1$ to get

$$
C_{w, \max }=g_{w}\left(\operatorname{Re}, F r,\left(\rho_{S}-\rho_{L}\right) / \rho_{L}, D_{J} / D_{T}, D_{p} / D_{T}\right)
$$

This describes the functional dependence - how variables and parameters are constrained as groups to depend on each other and hence how certain descriptions of behavior scale with length and time. That much we obtained above from dimensional analysis alone. But, the functional form of $g_{w}$ must be determined from experiments because the fundamental conservation equations are intractable, considering both the existence of particles and for turbulent flow. Of course, the functional form can be determined only to the extent that experiments "explore" the domain of the variables on which it depends. Realistically, only certain key aspects of the dependence could be determined from the relatively few variations of test parameters practical when operating the PEP during Phase I testing, which involves a single simulated waste.

\section{A.3.2.2 Example Dimensional Analysis of Empirical Information - Jet Suspension of Solids}

Data from experiments on suspending solids of density $\rho_{S}$, diameter $D_{p}$, and average mass fraction $C_{w 0}$ from the bottom of a tank of diameter $D_{T}$ by a jet with initial velocity $u_{0}$ from an orifice of diameter 
$D_{J}$ in a liquid of density $\rho_{L}$ and viscosity $\mu$ have been correlated ${ }^{1}$ in the form of the minimum velocity $u_{\min }$ required to just suspend the solids particles as

$$
u_{\min }=2\left(\rho_{S}-\rho_{L}\right) / \rho_{L}{ }^{2.08} \frac{v^{0.16} g^{0.42} D_{T}^{1.16} D_{p}^{0.1} C_{w 0}^{0.24}}{D_{J}}
$$

Rearranging,

$$
C_{w 0}^{0.24}\left(\left(\rho_{S}-\rho_{L}\right) / \rho_{L}\right)^{1.66}=\frac{1}{2}\left(\frac{D_{J}}{D_{T}}\right)^{0.84}\left(\frac{D_{p}}{D_{T}}\right)^{-0.1} \operatorname{Re}_{J}^{0.16} F r^{0.42}
$$

Solving for the mass fraction suspended,

$$
C_{w 0}=0.0557\left(\left(\rho_{S}-\rho_{L}\right) / \rho_{L}\right)^{-6.92}\left(\frac{D_{J}}{D_{T}}\right)^{3.5}\left(\frac{D_{p}}{D_{T}}\right)^{-0.42} \operatorname{Re}_{J}^{0.67} \mathrm{Fr}^{1.75}
$$

where $R e_{J}$ is the Reynolds number at the jet orifice: $\operatorname{Re}_{J}=u_{0} D_{J} / v$. That is, the characteristic velocity is the jet velocity. Then the correlation describes the minimum jet velocity that will suspend solids at the prescribed mass fraction. Therefore, also it is an expression for the maximum solids mass fraction that can be suspended at a prescribed jet velocity.

Thus, as expected from dimensional analysis, this correlation is of the form

$$
C_{w, \max }=g_{w}\left(\widetilde{\mathbf{x}}, \tilde{t}, \operatorname{Re}, F r, \rho_{L} /\left(\rho_{S}-\rho_{L}\right), C_{w 0}, D_{J} / D_{T}, D_{p} / D_{T}\right)
$$

But more importantly, the correlation obtained from a certain class of experiments includes the functional form, not just the functional dependence, for this class. Specifically, experimentalists found the maximum solids mass fraction depends very strongly on the particle density, both and as a separate parameter and as it affects the densimetric Froude number, which is

$$
F r=\frac{\rho_{L} u_{J}^{2}}{\left(\rho_{S}-\rho_{L}\right) g D_{T}}
$$

Note that if we vary the jet velocity and the size $D_{T}$ of the system while maintaining geometric similitude - i.e., while maintaining $D_{J} / D_{T}$ - and using the same particles—i.e., maintaining $D_{p}$ but not $D_{p} / D_{T}$-we have

$$
C_{w 0} \propto D_{T}^{0.16} u_{J}
$$

\footnotetext{
${ }^{1}$ Handbook of Industrial Mixing. 2004. Paul E, V Atiemo-Obeng, and S Kresta (Eds.), Section 10-3.4, "Suspension of Solids in Liquid-Jet Stirred Vessels," Wiley-Interscience, Hoboken, New Jersey.
} 
Therefore, for two geometrically similar systems to suspend the same mass fraction of the same solids, we must have

$$
I=\frac{\left(C_{w 0}\right)_{\text {large }}}{\left(C_{w 0}\right)_{\text {small }}} \propto \frac{\left(D_{T}^{0.16} u_{J}\right)_{\text {large }}}{\left(D_{T}^{0.16} u_{J}\right)_{\text {small }}} \quad \therefore\left(u_{J}\right)_{\text {small }}=\left(u_{J}\right)_{\text {large }}\left[\frac{\left(D_{T}\right)_{\text {large }}}{\left(D_{T}\right)_{\text {small }}}\right]^{1 / 6}
$$

That is, the velocity scales as $\operatorname{size}^{-1 / 6}(-0.15$ to the nearest inverse integer power) to suspend a specified solids mass fraction. Our best guess is that a similar relationship will describe the effect of pulse jet orifice velocities on suspending solids in UFP tanks of differing size scale.

An analogous analysis of the Zwietering Correlation ${ }^{1}$, describing the suspension of particles by rotating blade mixers, leads to essentially the identical $(-0.16$ instead of -0.15$)$ relationship: to suspend a specified solids mass fraction, the velocity scales as $\operatorname{size}^{-1 / 6}$, where the velocity is that of the blade tip.

\section{A.3.3 Density-Driven Resistance to Mixing}

Even if the settling velocity of particles is modest, it is possible for "clouds" of settling particles to form when suspended by a PJM pulse and then to descend very fast, resulting in little upward penetration of the cloud into the tank volume. This is a transient phenomenon; possibly correlations from steady-state tests cannot be applied even to approximate the effect.

Hence, it is worth considering how this effect "scales" with size and adjustable test parameters. There are three kinds of hydrodynamic processes to consider: 1) those suspending the solids from the bottom, 2) those affecting the rise and fall of the resulting solids cloud, and 3) those affecting the vertical uniformity of solids in a cloud. Apparently, within clouds the solids concentration has been uniform yet the cloud itself fails to mix with the surrounding liquid. The flow organization apparent in both the pulsedriven and gravity-driven flow durations suggests considering the pulse and gravitational work dominate the flow. To consider this, turbulent dissipation of energy should be ignored.

\section{A.3.3.1 Analysis}

Figure A.5 depicts a simple conceptual model. On the left is the hypothetical start of a PJM cycle, where solids are concentrated toward the bottom of a tank. On the right is the hypothetical situation at some point in the cycle where the solids have reached their maximum height and are about to descend due to bulk density differences under the effect of gravity (technically, a Taylor instability). Note the solids are not distributed horizontally uniformly. They rise toward the center in the test tank (which does not have the center volume excluded by a PJM support), displaying a uniform concentration within, and fall en masse with little mixing with surrounding liquid.

\footnotetext{
${ }^{1}$ Handbook of Industrial Mixing. 2004. Paul E, V Atiemo-Obeng, and S Kresta (Eds.), Section 10-3.1, ”JustSuspended Speed in Stirred Tanks," Wiley-Interscience, Hoboken, New Jersey.
} 


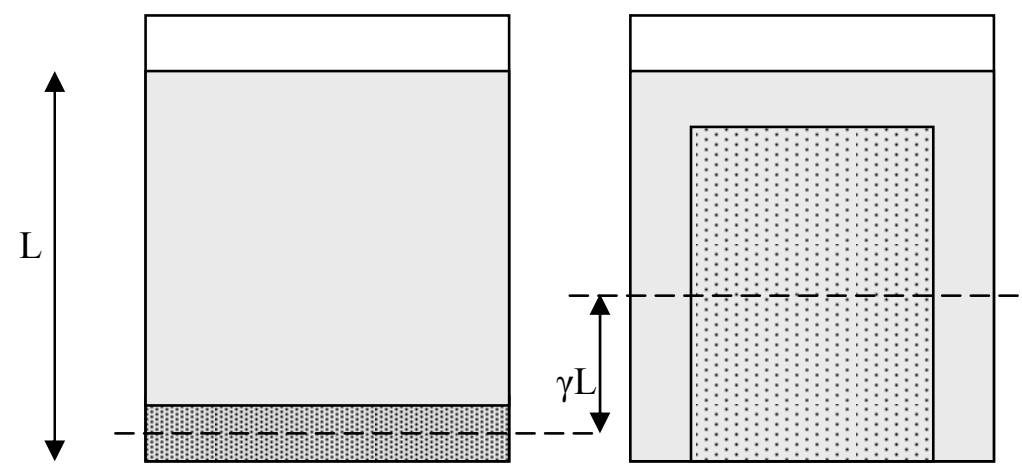

Figure A.5. Basic Elements of Density-Driven Transient Cycle

The cycle is a follows. Mass $m$ of solids initially resides near the bottom, then a pulse jet distributes the mass over a higher average elevation. The change in average elevation is $\gamma L$, where $L$ is the liquid height, taken to be the characteristic length of the system. Assume this change is repeated every PJM cycle. The work done in lifting the solids per cycle is

$$
W=\Delta \rho V_{S} g \gamma L
$$

where $V_{S}$ is the volume of solids and $\Delta \rho=\rho_{S}-\rho_{L}$. The work done by the pulse jet per cycle is the product (power)(time/cycle). The time per cycle is the drive time. The power due to the jet while it is driven is

$$
P=\rho_{L} u_{J}^{3} \frac{\pi}{4} \alpha^{2} L^{2}
$$

where $u_{J}$ is the pulse jet nozzle velocity and $\alpha$ is the ratio of the jet diameter to $L$. The ratio of the work done by the jet during a PJM cycle and the work done to lift the solids, in terms of $\gamma$, is

$$
\theta=\frac{\rho_{L} u_{J}^{3} \pi \alpha^{2} L^{2} t_{D}}{\Delta \rho V_{S} 4 g \gamma L}
$$

where $t_{D}$ is the drive time of the jet. The volume of the solids is

$$
V_{S}=\phi_{S} V=\phi_{S} \kappa L^{3}
$$

where $\varphi_{S}$ is the superficial solids volume fraction and $\kappa$ is a geometric factor describing the tank. Combining,

$$
\theta=\frac{\rho_{L} \pi \alpha^{2}}{\Delta \rho 4 \phi_{S} \kappa} \frac{u_{J}^{3} t_{D}}{g \gamma L^{2}}
$$




\section{A.3.3.2 Significance}

If we match the nozzle velocity between the PEP and PTF and reduce the drive time by the length scale ratio, then

$$
\frac{\theta_{P E P}}{\theta_{P T F}}=\frac{\frac{u_{J, P E P}^{3} t_{D, P E P}}{L_{P E P}^{2}}}{\frac{u_{J, P T F}^{3} t_{D, P T F}}{L_{P T F}^{2}}}=\frac{u_{J, P E P}^{3} t_{D, P E P} L_{P T F}^{2}}{u_{J, P T F}^{3} t_{D, P T F} L_{P E P}^{2}}=\frac{\left(t_{D, P T F} \frac{L_{P E P}}{L_{P T F}}\right) L_{P T F}^{2}}{t_{D, P T F} L_{P E P}^{2}}=\frac{L_{P T F}}{L_{P E P}}=4.5
$$

That is, the ratio of work done by the pulse jet in a cycle to the work required to lift the same solids in the same liquid to the same relative height $\gamma$ (ideally close to unity) is 4.5 greater in the current default PEP test than in the PTF. Thus, such tests would be non-conservative if cloud height is an issue. To represent the ability to raise the solids and thereby enable mixing nominally would require matching $u_{J}^{3} t_{D} / L^{2}$ between systems. Increasing $u_{J}$ or $t_{D}$, holding the other constant, probably would increase $\gamma$, tending to confound the "scale" relationship. Nevertheless, an energy balance points toward conditions to consider when interpreting PEP performance in terms cloud height. Specifically, the drive time is proportional to the volume displaced divided by the PJM volumetric flow rate, which is proportional to the product of the square of the nozzle diameter and the nozzle velocity. If the nozzle diameter is kept geometrically similar between systems, and the drive time scales as $\mathrm{L}^{3} /\left(\mathrm{L}^{2} \mathrm{u}\right)$ or $\mathrm{L} / \mathrm{u}$, then $\mathrm{u}^{3} t / \mathrm{L}^{2}$ scales as $\mathrm{u}^{2} / \mathrm{L}$.

A dimensional analysis of the general effect of stresses caused by bulk differences in density is included in Section A.4.2, which identifies the governing dimensionless group as the Froude number, which is proportional to $\mathrm{u}^{2} / \mathrm{L}$. Hence, we find ultimately the effect of a bulk density difference on the flow behavior can be represented in terms of the Froude number, as predicted by dimensional analysis. Matching the Froude number is equivalent to matching $\mathrm{uL}^{-1 / 2}$ between systems.

Because this phenomenon involves self-organization of a density-driven flow, concerns about effects on mixing could be moot if the PJM tanks and other occlusions disrupt the initial upward flow sufficiently to prevent organized density differences to persist over a PJM cycle, in which case this phenomenon would not be important. However, in lieu of PEP data, one can only assume this.

\section{A.4 Hydrodynamics}

\section{A.4.1 Rheology}

Rheology is the study of the relation between fluid motion and the forces that drive fluid motion; the relationship is often termed simply the rheology of the fluid. Many common fluids are described by Newton's Law: that the rate of strain, i.e., the velocity gradient, is proportional to the shear stress driving the flow. For a simple two-dimensional flow with only one velocity component varying in only one direction, Newton's law takes its simplest form,

$$
\tau_{y x}=\mu \frac{d u_{x}}{d y}
$$


where $u_{x}$ is the velocity component in the $x$-direction, $y$ is a direction perpendicular to $x$, and $\tau_{y x}$ is the shear stress in the fluid acting in the $x$-direction on surfaces aligned perpendicular to the $y$-direction. The velocity gradient (derivative) is the strain rate and has units of $1 /$ time.

Process slurries in the WTP can be non-Newtonian for sufficiently large solids concentrations, denoting that such fluids do not obey Newton's law of viscosity. There are many models of such behavior; the fluids of interest here tend to be either Newtonian fluids or "Bingham plastics," which are fluids that behave as Newtonian fluids except the strain rate is proportional to the shear stress reduced by a "yield stress" that must be attained before any flow occurs. That is, for shear stresses less than the yield stress, the fluid does not move (does not "yield"). The stress-strain relationship corresponding to the flow field invoked above is

$$
\tau_{y x}-\tau_{0}=\mu \frac{d u_{x}}{d y}
$$

Put in terms of the strain rate as a function of the shear stress,

$$
\frac{d u_{x}}{d y}=\frac{\tau_{y x}-\tau_{0}}{\mu_{0}}
$$

In addition, Bingham plastic fluids can exhibit a shear strength, $\tau_{s}$. This differs from the yield stress, $\tau_{0}$, discussed above, which is a parameter describing the stress-strain relationship for a flowing fluid. However, a Bingham plastic that has been stagnant for some time essentially "sets up" as a very weak solid that can be deformed only after experiencing a shear stress equal to the shear strength, which is substantially greater than the yield stress. The stress-strain history for a Bingham plastic would be as depicted, conceptually, in Figure A.6, where the "one-way" dashed line depicts the stress during the initial strain of a stagnant fluid, and the solid line depicts the subsequent stress-strain relationship, valid for either increasing or decreasing strain so long as a strain rate is maintained. The relative magnitude of the yield strength and yield stress can vary widely and may not be as dramatic as depicted.

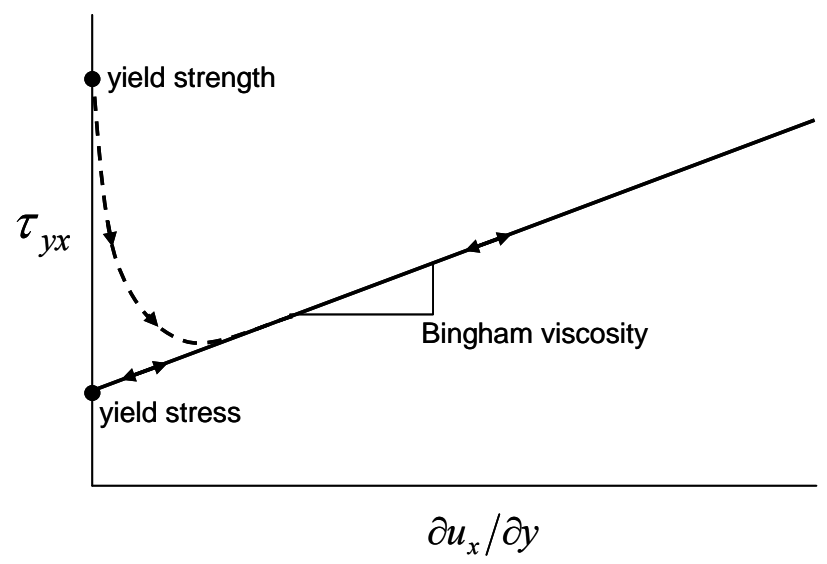

Figure A.6. Stress-Strain History for a Bingham Plastic

No strain rate is possible until $\tau_{y x}>\tau_{s}$. Once this is attained, the fluid moves and decreases the shear strength to an asymptotic value, the yield stress, that applies until the fluid come to rest. At rest, the fluid 
"sets up" again. If enough time passes, the full shear strength is recovered. If little time passes, some stress between the shear strength and the yield stress must be attained to restart fluid motion.

\section{A.4.2 Characteristic Stresses}

Forces acting on single- and multiple-phase fluid systems can be described in terms of mechanical stresses, force/area, and categorized as follows in terms of an imposed characteristic velocity $u$ and a characteristic length $L$ together with equilibrium fluid properties: density, viscosity, yield stress, yield strength, and interfacial tension:

- Inertial stress, $\rho u^{2}$, is the normal stress (pressure) differential associated with stopping a flowing fluid

- Viscous stress, $\mu(u / L)$, results from fluids resisting a strain rate

- Yield stress or shear strength, $\tau_{0}$ or $\tau_{s}$, that must be applied to a Bingham plastic for it to continue to flow or begin to flow

- Gravitational stress, $4 \rho g L$, resulting from the gravitational acceleration of two adjacent phases with different densities

- Capillary stress, $\sigma / L$, resulting from a curved interface between two phases, where $\sigma$ is the interfacial tension.

The ratios of these stresses are dimensionless groups as named in Table A.2.

Table A.2. Dimensionless Groups

\begin{tabular}{|c|c|c|c|}
\hline 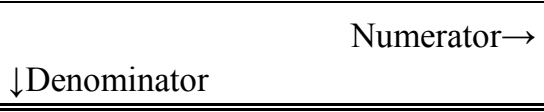 & Inertial $\rho u^{2}$ & Viscous $\mu \frac{u}{L}$ & $\begin{array}{c}\text { Gravitational } \\
(\Delta \rho) g L \\
\end{array}$ \\
\hline \multicolumn{4}{|l|}{ Inertial $\rho u^{2}$} \\
\hline Viscous $\mu \frac{u}{L}$ & $\begin{array}{c}\mathrm{Re} \\
\text { (Reynolds Number) }\end{array}$ & & $\begin{array}{c}\mathrm{Ga} \\
\text { (Gravity Number) }\end{array}$ \\
\hline Gravitational $(\Delta \rho) g L$ & $\begin{array}{c}\text { Fr } \\
\text { (Froude Number) }\end{array}$ & & \\
\hline Capillary $\frac{\sigma}{L}$ & $\begin{array}{c}\text { We } \\
\text { (Weber Number) }\end{array}$ & $\begin{array}{c}\mathrm{Ca} \\
\text { (Capillary Number) }\end{array}$ & $\begin{array}{c}\text { Bo } \\
\text { (Bond Number) }\end{array}$ \\
\hline Yield Stress/Shear Strength $\tau_{0}, \tau_{s}$ & $\begin{array}{c}\operatorname{Re}_{\tau}, \operatorname{Re}_{\mathrm{s}} \\
\text { (Yield/Shear Strength } \\
\text { Reynolds Number) }\end{array}$ & - & - \\
\hline
\end{tabular}

The significance of these groups to the similitude between the PEP and the WTP includes the following.

- Re: Reynolds number- $\frac{\rho u L}{\mu}$-pertains to competition between flow-destabilizing inertial stresses and flow-stabilizing viscous forces; the greater the Reynolds number, the greater the tendency for turbulence. This is important at the scale of recirculating flows in tanks and at the scale of the flow of liquid around a rising bubble or a settling solid particle. 
- $\operatorname{Re}_{\tau}$ : Yield Reynolds number- $\frac{\rho u^{2}}{\tau_{0}}$-where $\tau_{0}$ is the yield stress of a Bingham plastic - pertains to the dissipation of kinetic energy dissipation due to work done against the yield stress; affects the turbulent strain rate caused by kinetic energy dissipation.

- $\mathrm{Re}_{\mathrm{s}}$ : Shear Strength Reynolds number- $\frac{\rho u^{2}}{\tau_{s}}$-where $\tau_{\mathrm{s}}$ is the shear strength of a Bingham plasticpertains to the ability of jets to penetrate stagnant regions of a Bingham plastic fluid- $-\operatorname{Re} \tau \sim 1$ at the velocity near the boundary of a mixing cavity.

- Bond (or Eotvos) number- $\frac{\Delta \rho g L^{2}}{\sigma}$ - pertains to competition between gravitational forces tending to collapse the roof of bubbles (because fluid above is denser than the gas below) and capillary forces tending to resist deformation of the sphericity of a bubble. This sets the maximum stable size of bubbles, which rise as spherical caps.

- Froude number $-\frac{\rho u^{2}}{\Delta \rho g L}$ - pertains to competition between inertial stresses tending to resist fluid accelerating into PJM cylinders during refill and gravitational forces tending to accelerate the fluid into them; the competition determines the magnitude of the refill velocity at the PJM orifices (modified by imposing a pressure difference at the surface of the liquid in the cylinder). The Froude number also describes the competition between buoyancy forces acting to push a bubble upward and inertial forces - i.e., drag forces - acting to resist motion, which determines the rise velocity of large, spherical cap bubbles.

- Gravity number- $\frac{\Delta \rho g L^{2}}{u \mu}$ pertains to competition between gravitational stresses tending to move fluids due to density imbalances, such as resulting from temperature gradients, and viscous stresses resisting the motion; the competition determines the magnitude of the resulting fluid velocity.

\section{A.4.3 Characteristic Velocities}

\section{A.4.3.1 Forced Convection}

For systems undergoing "forced" convection, a characteristic velocity is imposed on the fluid at some point, such as the velocity of a jet at its orifice or the velocity of the tip of an agitator blade. In such cases, the characteristic velocity invoked in the characteristic forces noted above is just this forced velocity; i.e., a hydrodynamic boundary condition.

\section{A.4.3.2 Free (Thermal) Convection}

For systems undergoing "free" convection, no velocity is imposed on the system. Instead, a density difference is imposed as a boundary condition through differences in temperature or concentrations. Then 
the magnitude of the resulting velocities adjusts such that gravitational and viscous forces are comparable; i.e., such that the Gravity number is of an order of unity. That is,

$$
u \sim \frac{(\Delta \rho) g L^{2}}{\mu}
$$

The density difference due to temperature differences is

$$
\Delta \rho=\frac{d \rho}{d T} \Delta T=\rho \beta_{T} \Delta T \quad \beta_{T} \equiv \frac{d \ln \rho}{d T}
$$

Substituting

$$
u \sim \frac{\rho \beta_{T} g L^{2} \Delta T}{\mu}
$$

At this velocity, the Reynolds number is

$$
\operatorname{Re}=\frac{\rho u L}{\mu} \sim \frac{\rho^{2} \beta_{T} g L^{3} \Delta T}{\mu^{2}}=G r
$$

where Gr is the Grashof number, which, being a form of the Reynolds number, describes the tendency for thermally driven free convection to become turbulent. If instead, the free convection is driven by concentration differences, then

$$
G r=\frac{\rho^{2} \beta_{C} g L^{3} \Delta C}{\mu^{2}} \quad \beta_{C}=\frac{d \ln \rho}{d C}
$$

The analysis is complicated further by the existence of a yield stress in a non-Newtonian fluid. Assuming that density-induced stresses arise that are greater than the yield stress, the resulting fluid motion will be less than that predicted from correlations based on Newtonian fluids. At a minimum, one would need to define an effective Grashof number. Any need for such corrections and, if needed, the corrections themselves, will have to be inferred from any substantial difference between predicted and actual behavior in the PEP.

\section{A.4.3.3 Particle Terminal Velocity}

The solid particles in the WTP slurry are small enough that their motion relative to the surrounding fluid is determined by shear stresses compared to the gravitational force. For Newtonian fluids, this is described by Stokes law:

$$
u=\frac{\delta^{2} \Delta \rho g}{18 \mu}
$$


where $\delta$ is the size of the particle, and $\Delta \rho$ is the difference in density between the particle and the fluid. This is because the shear force is the product of the area and the shear stress, $\delta^{2} \mu(u / \delta)$, and the buoyancy force is proportional to $\Delta \rho \delta^{3} g$. Equating these, the terminal velocity is proportional to

$$
u \propto \frac{\Delta \rho g \delta^{2}}{\mu}
$$

which is essentially Stokes law for settling. In a Bingham plastic fluid, the order of the shear stress is

$$
\tau_{*}+\mu(u / \delta)
$$

where $\tau_{*}$ is $\tau_{0}$ if the fluid is not stagnant - i.e., the case of settling within a sheared fluid —and is $\tau_{s}$ if the fluid is stagnant. Then the order of the terminal velocity is

$$
u_{\text {particle terminal }} \sim \frac{\delta}{\mu} \max \left(\Delta \rho \delta g-\tau_{*}, 0\right)
$$

That is, the terminal velocity is zero unless

$$
\delta>\delta_{\text {critical }} \frac{\tau_{*}}{\Delta \rho g}
$$

For Newtonian fluids, $\tau_{*}$ is zero and some settling always occurs, although not necessarily of any significance. In a Bingham plastic fluid, particles below the critical size $\delta_{\text {critical }}$ will not settle.

\section{A.4.3.4 Bubble Terminal Velocity}

Air injected to sparge the liquid forms bubbles that rapidly expand as they rise due to the decreasing surrounding hydrostatic pressure combined with continual equilibration with the vapor pressure of water. As they expand, capillary forces tend to stabilize the bubbles while gravitational forces tend to destabilize them because the fluid above the bubble is denser than the bubble-i.e., as the bubble grows in size, eventually the "roof caves in" to create a toroidal bubble because capillary forces can no longer resist gravitational forces, and the top surface of the bubble becomes unstable. Once a toroid forms, it quickly breaks into smaller bubbles. Thus, the stable size of the bubble is determined by the ratio of gravitational to capillary forces, which is the Bond number. For bubbles, this ratio is often termed the Eotvos number; it is

$$
E o=\frac{\Delta \rho g d^{2}}{4 \sigma}
$$

where $\Delta \rho=$ density difference between the gas and the liquid

$G=$ acceleration of gravity

$D=$ bubble diameter

$\Sigma=$ surface tension of the liquid. 
At the limit of their stable size, bubbles exist as spherical caps, and as they rise, the flow around them is turbulent, such that the drag force and hence the rise velocity essentially does not depend on viscosity. Joseph $^{1}$ shows that for spherical cap bubbles in this domain, the Froude number (the ratio of inertial to gravitational forces) based on the terminal velocity of the bubble-i.e., the velocity of the bubble relative to the fluid enclosing it-is constant and equal to $\sqrt{2} / 3$. That is,

$$
\mu=0 \quad u=\frac{\sqrt{2}}{3} \sqrt{g d}
$$

where $u$ is the terminal velocity. Then the velocity can be written in terms of the Eotvos number by substituting for the bubble diameter to get

$$
u=\left(\frac{8}{9} \frac{g \sigma}{\rho} E o\right)^{1 / 4}
$$

Joseph reports that a numerical study by Boulton-Stone et al. ${ }^{2}$ suggests that spherical cap bubbles are stable only when the Eotvos number based on an equivalent spherical radius is less than about 32 . For higher values of Eo, an unstable toroidal bubble is formed before breakup. Substituting for the Eotvos number,

$$
u \sim\left(\frac{256}{9} \frac{g \sigma}{\rho}\right)^{1 / 4}=2.3\left(\frac{g \sigma}{\rho}\right)^{1 / 4}
$$

For either water or WTP process liquids, this is on the order of $1 \mathrm{ft} / \mathrm{s}$. The above analysis is based on observations and correlations for single bubbles rising in an otherwise stagnant liquid, in which case, the rise in velocity does not exhibit any inherent proportionality to the characteristic velocities imposed on the PEP, WTP, PJMs or pumps. This should also be true for a column of bubbles rising due to their buoyancy. The situation is more complicated if a forced flow field is superimposed; in that case, it is best to assume that the fluid motion induced by rising bubbles adds simply to any velocity imposed by PJMs or pumps.

Also, the above analysis is based on observations and correlations for bubble rising in Newtonian fluids. The existence of a yield stress or shear strength will impede the bubble rise, but if the yield Reynolds number is on the order of the viscous Reynolds number for which spherical bubble caps exist in Newtonian fluids ( $\operatorname{Re} 100)$, probably the behavior in non-Newtonian fluids will be similar, and the above analysis provides at least an order-of-magnitude analysis and the proper functional forms.

\section{A.4.3.5 Mixing Caverns}

As is shown below, the penetration of jets into stagnant regions of a Bingham plastic fluid exhibiting a shear strength depends on the shear-strength Reynolds number. This penetration quantifies the formation of any "mixing cavern." Therefore, to make the existence and extent of mixing caverns in the

\footnotetext{
${ }^{1}$ Joseph DD. 2003. "Rise Velocity of Spherical Cap Bubble." Journal of Fluid Mechanics 488:213-223.

2 Boulton-Stone JM, PB Robinson, and JR Blake. 1995. "A Note on the Axisymmetric Interaction of Pairs of Rising, Deforming Gas Bubbles.” International Journal of Multiphase Flow 21:1237-1241.
} 
PEP represent expected behavior in the PEP, the shear strength Reynolds number must be matched. Given the same solids, particle-size distribution, and liquid composition to provide representative solids for leaching, the shear strength will be the same for materials in the PEP as for materials in the WTP. Therefore, jet velocities (filter loop recirculation entrance, PJM orifice) are matched between the PEP and the WTP so that the shear-strength Reynolds number is matched.

\section{Jet Penetration}

The processes limiting the streamwise extent of a jet can be idealized in several ways.

1. If the jet is confined transversely by a rigid boundary that imposes a shear stress at the periphery of the jet, the momentum decreases along its length according to

$d J=-\tau_{\Gamma}(z) \Gamma(z) d z$ or $J=\rho u_{0}^{2} A_{0}-\int_{0}^{z} \tau_{\Gamma}(z) \Gamma(z) d z$ until the jet is subsumed as the center velocity becomes small compared to nearby velocities imposed by other processes. The peripheral shear stress scales as $\tau \sim \mu(u / L)$, where $\mu$ is the viscosity of the fluid. Then the ratio of the momentum flux $\mathrm{J}$ to the flux at the origin of the jet is given by

$$
\begin{aligned}
& \therefore \frac{J}{\rho u_{0}^{2} A_{0}}=1-\int_{0}^{z}\left(\frac{\tau_{\Gamma}(z)}{\rho u_{0}^{2}}\right)\left(\frac{\Gamma(z / L)}{L}\right)\left(\frac{L^{2}}{A_{0}}\right) d(z / L)=\Lambda(\eta)=1-\frac{1}{\operatorname{Re}_{0}} \int_{0}^{\eta} \frac{\psi(\eta) \phi(\eta) \gamma(\eta)}{\alpha_{0}} d \eta \\
& \Lambda(\eta)=\frac{J(g h)}{J_{0}} \quad \eta=\frac{z}{L} \quad \psi(\eta)=\frac{\tau(\eta)}{\mu\left(u_{0} / L\right)} \quad \phi(\eta)=\frac{u(\eta)}{u_{0}} \quad \gamma(\eta)=\frac{\Gamma(\eta)}{L} \quad \alpha_{0}=\frac{A_{0}}{L^{2}} \quad \operatorname{Re}=\frac{\rho u_{1} L}{\mu}
\end{aligned}
$$

where $U_{0}=$ velocity at the start of the jet

$L=$ characteristic length scale for the system in which the jet is embedded

$A_{0}=$ jet area at the origin

$\Gamma=$ length of the periphery of the jet over which shear stress occurs.

The shear stress will scale with the system as $\mu(u / L)$, the periphery will scale as $L$, the velocity will scale as $u_{0}$, and the jet area will scale as $L^{2}$; therefore, the dimensionless terms $\Lambda, \psi, \varphi, \gamma$, and $\alpha_{0}$ are independent of scale. Therefore, the ratio of the momentum flow compared to the initial value decays along the extent of the jet relative to the length scale as a function of the jet Reynolds number, Re. The greater this Reynolds number, the greater the extent of the self-similar region of the jet compared to the length scale of the system. For large jet Reynolds numbers, the jet will extend until deflected by a boundary, in which case the location of the disruption is determined by the tank geometry instead of hydrodynamic processes occurring in the jet.

2. The jet and parallel jets expand until they transversely bound each other. In that case, the area can no longer increase, and hence the velocity does not decay. However, together the jets form a larger jet that is more likely to encounter rigid boundaries at its edge, causing the jet to decay as noted above. Because the width of a self-similar jet expands proportionately to the ratio of the distance from the origin to the orifice diameter, and the orifice diameter is in a specified proportion to the length scale, two jets will merge at a location determined by the tank geometry. Hence, if the arrangement of jet orifices preserves geometric similitude, the flow patterns of jets will be geometrically similar.

3. The jet can be dramatically disrupted by impinging on a complex boundary (e.g., the boundaries of features inside a tank) that essentially divides the jet into many jets proceeding in different directions. Practically, this amounts to converting a jet to unstructured turbulence. The location of the disruption 
is the location of the complex boundary. Consequently, the extent of the jet is determined by the geometry of rigid boundaries in the tank. Hence, if the boundaries preserve geometric similitude, the extent of jets will be geometrically similar.

4. In a non-Newtonian fluid, even if the momentum flow is sustained, the jet can expand until the center velocity is too small to induce inertial stresses equal to the shear strength of surrounding stagnant fluid. At that point, the surrounding fluid acts a rigid boundary forcing the jet to spread and decay as though impinging on a wall, and we have

$$
\tau_{s}=\rho u^{2}=\rho u_{0}^{2} \sqrt{\frac{A_{0}}{A_{1}}} \quad \therefore \frac{A_{1}}{A_{0}}=\operatorname{Re}_{s}^{2}
$$

where $A_{0}$ and $A_{1}$ are the jet cross-sectional area at the start (" 0 ") and some location " 1 " at which the jet effectively ends, and as noted above $\mathrm{Re}_{\mathrm{s}}$ is the shear strength Reynolds number.

5. Because the ratio of the jet area to the initial area for a self-similar jet is a function of the distance along the jet compared to the orifice diameter that is proportional to the length scale of the system, the extent of the jet relative to the length scale is a function of the yield Reynolds number.

\section{Significance of the Shear Strength Reynolds Number}

For 1, 2, and 3 above, the extent of the jet is determined by the tank geometry. Therefore, the extent of the jet compared to the length scale will be the same for systems that are geometrically similar, which is true for the PEP compared to the WTP. For 4 above, this is also true if the shear strength Reynolds number is matched between systems.

The potential existence and size and shape of any mixing cavity is defined by the penetration of jets into fluids exhibiting a shear strength. From the above, this penetration, and hence the size and shape of any mixing cavity, depends primarily on the shear strength Reynolds number. The processes leading to the size and shape of a mixing cavity are too complex to calculate or otherwise estimate. Therefore, we must rely on similitude between the PEP and the WTP to match the existence, size, and shape of mixing cavities in the PEP and the WTP. To this end, one must match the shear strength Reynolds number between the PEP and the WTP. Consequently, given the same rheological properties of the slurry in the PEP and the WTP, one must match velocities at the origins of jets between the PEP and the WTP, including notably the filter loop recirculation jet and PJM jets. Consequently, the ratio of the hydrodynamic time scale, i.e., length/velocity, is the ratio of the length scales, and hence hydrodynamic events and processes in the PEP should occur proportionately faster than in the WTP when the corresponding jet velocities are matched.

\section{A.5 Heat Transfer}

\section{A.5.1 Scaling Analysis}

A scaling analysis of heat transfer associated with the scaled and unscaled UFP vessels may be reasonably divided into processes internal to the vessel and those from the vessel wall outwards. By constructing an analytical apparatus to understand the external heat transfer, this information can then serve as a global boundary condition to the internal heat-transfer processes. 
The external heat-transfer problem may be loosely subdivided into three smaller problems.

- The first is natural convection ${ }^{1}$ over the outer surface of the vessel with an associated radiative exchange between the vessel exterior and its surroundings. The Nusselt number associated with natural convection generally scales with the Rayleigh number to some exponent. If the flow is laminar or turbulent, the exponent is one quarter or one third, respectively. The form and coefficients of such correlations depends also on the orientation of the surface relative to vertical.

- The second is forced convection through the side and bottom jackets. In the laminar case, Nusselt numbers are essentially constant after a development phase. For the turbulent case, at fixed Prandtl number, the transfer scales approximately as the Reynolds number (based on the pipe diameter) raised to the power 4/5 (this is a classic result; for example, see White ${ }^{2}$ )

- Third is heat exchange between the vessel and all piping attachments, either through conduction along the pipes or via the flux of energy into or out of the vessels by fluid convection. This contribution is more difficult to assess because it depends on the rates and conditions of any flows as well as the temperature gradients along the pipe axes.

The internal heat transfer is generally less amenable to scaling analyses than is the external problem. It includes the following.

- The slurry within the vessel may exchange heat with its surroundings: the vessel walls and the gas phase above. Slurries may be either Newtonian or non-Newtonian. As temperature differences are generally small within the vessel, net radiative exchange is likely minimal. Heat is exchanged convectively between the vessel walls and the slurry. This occurs within boundary layers, and periodic stagnation point flows directly adjacent to PJM exhaust nozzles.

- Slurry energy may be changed via both conduction through the piping, which connects the vessel to the outside world, and by convection-induced enthalpy changes caused by the flux of material, through the piping, into or out of the vessel.

- PJMs provide kinetic energy to the slurry, which is transformed into heat via viscous dissipation. Work done by the fluid on the inside mixer walls also adds energy to the slurry. Energy associated with the suction process must also be included. Additional viscous dissipation arises to the slurry from viscous dissipation associated when the recirculation pumps are in operation.

- Temperature changes are caused by chemical reactions and phase changes. Beyond the chemical reactions inherent in the processing of the waste, whether exothermic or endothermic, evaporation/ sublimation and condensation occur. Eductor tubes introduce hot steam at $172^{\circ} \mathrm{C}$ while sparger tubes introduce dry, room-temperature air. These will give rise to condensation or vaporization of the water. Vaporization will occur into dry air bubbles until there is a partial pressure balance of the water between the bubble and the adjacent slurry. Both of these processes may give rise to local temperature inhomogeneities.

- Radioactive material imparts energy as decay heat.

\footnotetext{
${ }^{1}$ The Rayleigh Number scales as the length cubed. Similar dependencies of the Nusselt Number on the Rayleigh number for laminar versus turbulent flow are observed for various free-convection domains. A useful reference is: B Gebhart, Y Jaluria, RL Mahajan, and B Sammakia. 1988. Buoyancy-Induced Flows and Transport, Hemisphere Publishing Company, New York.

${ }^{2}$ White FM. 2006. Fluid Mechanics, $6^{\text {th }}$ Edition, McGraw-Hill Higher Education.
} 
It is important to distinguish between the concepts of heat transfer and temperature distribution. Heat transfer is traditionally associated with the heat flux vector whereas temperature distributions focus on the entire energy equation. Even with a vanishing heat flux vector, energy can be transported simply by convection. In this context, mixing causes a large-scale advection of energy followed by conduction processes occurring at small scales. Conduction processes are often insufficient to achieve a uniform temperature field and must be supplemented with convective/mixing processes. Both the PJMs and buoyancy-driven flows (and possibly the sparger bubbles) are mixing agents, causing the gradients to steepen and increase the volume in which significant gradients exist. Recent computational fluid dynamic simulations for a Newtonian fluid suggest that the slurry will be nearly isothermal.

A particular aspect of this study that merits mention is the fact that the principal item being scaled in these studies is the characteristic length scale. Between the scaled and unscaled UFP vessels, the change in the characteristic length scale is slightly greater than half an order of magnitude, i.e., there is little scale separation between the full- and reduced-scale vessels. This potentially gives rise to a situation where uncertainties in proportionalities can be of similar magnitude to changes in other terms. For instance, laminar natural convection occurring over spheroids is well described by

$$
N u \sim R a^{1 / 4} \sim L^{3 / 4} \quad R a=G r \operatorname{Pr}
$$

Even for well-studied flows, the proportionality constant has a wide variation in the literature. If a UFP vessel is scaled by a factor of 4.5, the Nusselt number would change by a factor of 3.1. However, even in canonical flow fields, there is uncertainty in the proportionality coefficients. Estimating heat transfer in real engineering environments by appealing to idealized results adds further uncertainty to the proportionality coefficients that could approach a factor of three. For this reason, there is not enough scale separation to allow proportionalities to be ignored.

A general caveat to the scaling analysis is that it is incapable of transparently alerting readers as to when a physical process moves from one regime to another. For instance, an order of magnitude change in the Reynolds number may be the difference between a laminar and turbulent boundary layer. A small change in temperature may send a material through a phase change where completely different empirical correlations apply. The problem is that these transition points can be somewhat context dependent.

\section{A.5.2 Heat Transfer Across Tank Boundaries}

Enthalpy flow as heat transfer through the boundary of the tank, distinct from enthalpy added by steam injection or removed by vaporization due to sparging, is a complex process driven by the difference between the average temperature of the fluid in the tank and the temperature outside the tank and involving various heat-transfer resistances in parallel and in series. Enthalpy lost by vaporization is replaced by condensate, which by itself causes essentially no net addition of mass. Furthermore, the enthalpy flow between steam injection and vaporization by sparging is expected to cause small temperature differences in the fluid, as discussed below. However, enthalpy lost by heat transfer across the boundary must be replaced by injecting additional steam, which provides a net addition of water to the tank. Heat transfer across the boundary can be represented conceptually as shown Figure A.7; no attempt is made to depict the actual location of heat-transfer processes within the boundary. 


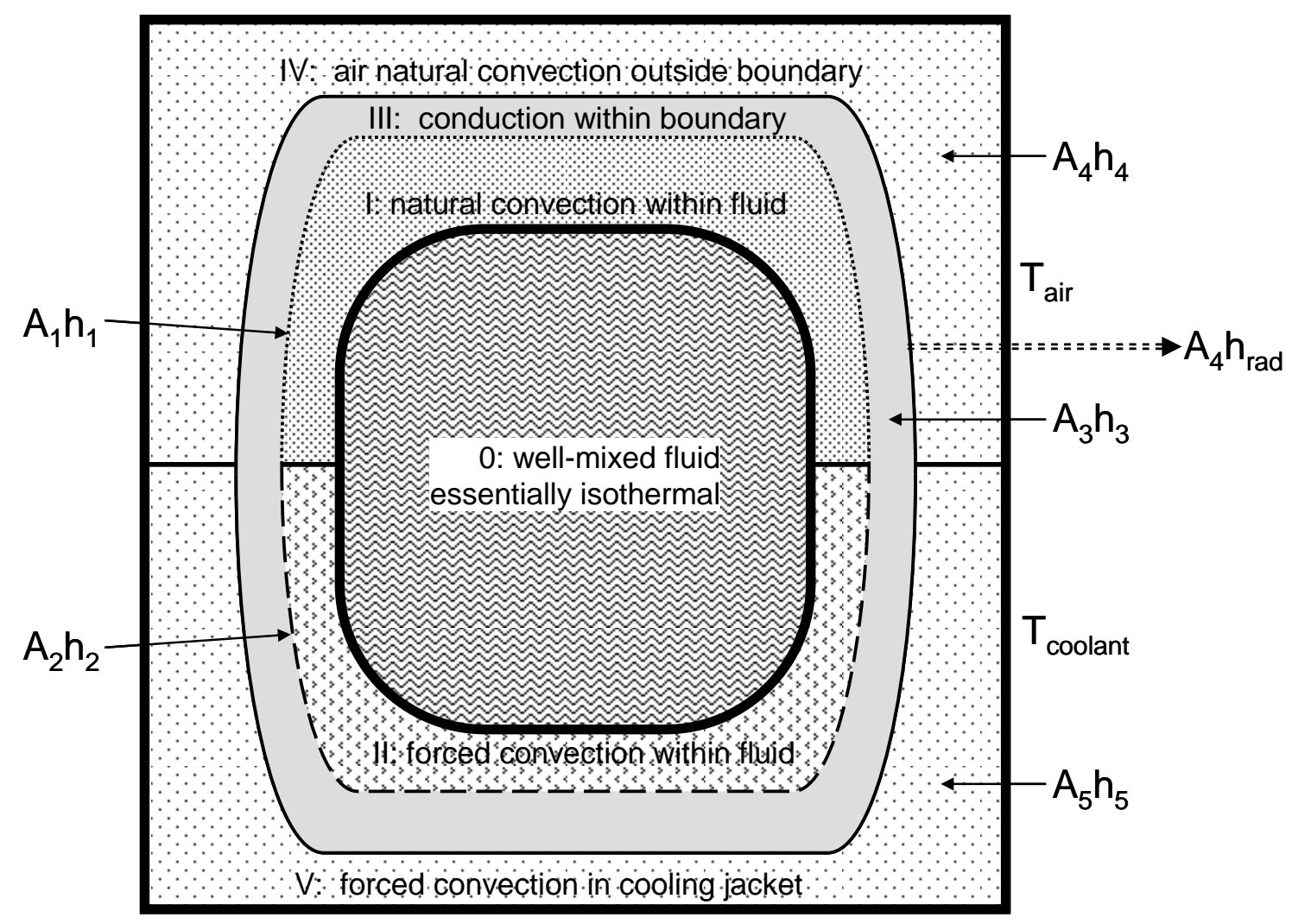

Figure A.7. Heat Transfer Across the Boundary

Region 0 in Figure A.10 is essentially isothermal well-mixed fluid within the tank. Region I is fluid undergoing natural convection inside the tank. Region II is fluid undergoing forced convection (e.g., from PJMs or recirculation) inside the tank. Both Region I and II could include some stagnant fluid if a significant yield stress is exhibited by the fluid, which would decrease the heat-transfer coefficients. Region III is the tank wall. Region IV is air outside the tank undergoing natural convection. Region V is the coolant in the cooling jacket. In addition, radiation heat transfer occurs in parallel over area $\mathrm{A}_{4}$.

To make the analysis tractable while retaining relevance to the actual, complex heat-transfer paths, consider heat transfer in parallel in Regions I and II and in parallel in Regions IV and V, and the combined heat transfer in Regions I/II and IV/V in series with the heat transfer in Region III. Also, the temperature difference across Region I is the same as for II, while the temperature difference across Region V can be controlled independently of that for Region IV because the coolant temperature can be controlled independently of the ambient temperature, $\mathrm{T}_{\text {air }}$. Associated with each region is a temperature difference $\Delta T$ across it, a heat-transfer area $A$, and a heat-transfer coefficient $h$.

Because the tank boundary is thin and more conductive than the other regions, the temperature difference across it will be relatively small enough to neglect for the purposes of this analysis and hence can be characterized simply by a temperature. 
For this conceptual analysis, there is no basis for associating fractions of areas $A_{1}$ or $A_{2}$ with fractions of areas $A_{4}$ or $A_{5}$. Instead, we simply equate the sum of the heat fluxes through Regions I and II with the sum through Regions IV and V and also consider the temperature of Region III to be an average over $A_{3}$, i.e., $T_{3}$. Then the heat flows are

$$
\begin{aligned}
& \dot{Q}_{1}=h_{1} A_{1}\left(T_{0}-T_{3}\right) \\
& \dot{Q}_{2}=h_{2} A_{2}\left(T_{0}-T_{3}\right) \\
& \dot{Q}_{4}=\left(h_{4}+h_{\text {rad }}\right) A_{4}\left(T_{3}-T_{\text {air }}\right) \\
& \dot{Q}_{5}=h_{5} A_{5}\left(T_{3}-T_{\text {coolant }}\right) \\
& \dot{Q}_{1}+\dot{Q}_{2}=\dot{Q}_{4}+\dot{Q}_{5}
\end{aligned}
$$

where $h_{\text {rad }}$ is a linearized radiation heat-transfer coefficient that does not depend on the length scale. This form is sufficient when, as in this case, the temperature difference is small compared to the average absolute temperature. Solving for the temperature at the boundary and the total heat flux,

$$
\begin{aligned}
& T_{3}=\frac{\left(h_{1} A_{1}+h_{2} A_{2}\right) T_{0}+\left(h_{4}+h_{\text {rad }}\right) A_{4} T_{\text {air }}+h_{5} A_{5} T_{\text {coolant }}}{\left(h_{4}+h_{\text {rad }}\right) A_{4}+h_{1} A_{1}+h_{2} A_{2}+h_{5} A_{5}} \\
& \dot{Q}=\dot{Q}_{1}+\dot{Q}_{2}=\gamma\left(h_{4}+h_{\text {rad }}\right) A_{4}\left(T_{0}-T_{\text {air }}\right)+\gamma h_{5} A_{5}\left(T_{0}-T_{\text {coolant }}\right) \quad \gamma=\frac{h_{1} A_{1}+h_{2} A_{2}}{h_{2} A_{2}+h_{1} A_{1}+\left(h_{4}+h_{\text {rad }}\right) A_{4}+h_{5} A_{5}}
\end{aligned}
$$

For a Newtonian fluid, the heat-transfer coefficients take the form

$$
h=\frac{k}{L} N u
$$

where $k$ is the thermal conductivity of the fluid (liquid in the tank, air outside), $L$ is a characteristic length, and $N u$ is the Nusselt number, a dimensionless heat transfer coefficient. For forced convection, $N u_{2}=$ $\left(k_{f} / L\right) f(R e, P r)$, where $k_{f}$ is the thermal conductivity of the fluid, and Re is the Reynolds number based on the flow forcing the heat transfer. Typically, $N u=($ constant $) \operatorname{Re}^{a} \operatorname{Pr}^{b}$, which for constant characteristic velocity scales as $L^{a}$, where $a$ is typically $1 / 2$ to $3 / 4$. For natural convection, $N u_{1 \text { or } 4}=(k / L) f(G r$, $P r)$, where $G r$ is the Grashof number associated with the natural convection domain, and typically $N u=($ constant $) G r^{c} P r^{d}$, which for constant characteristic temperature difference scales as $L^{3 c}$ where typically $c$ is $1 / 4$ to $1 / 3$. Hence, we can approximate the Nusselt numbers to be roughly proportional to $L$. Then the heat-transfer coefficients are weak functions of the length scale, and hence,

$$
\dot{Q} \propto A \propto L^{2}
$$

which is a crude but instructive evaluation of the order of the dependence of the heat loss on the length scale at steady state.

\section{A.5.3 Effect of Sparger Evaporation on Temperature Uniformity}

Consider the heat transfer between the point of steam injection and the top of the zone of vaporization in the combined sparge bubble regions, as shown in Figure A.8. 


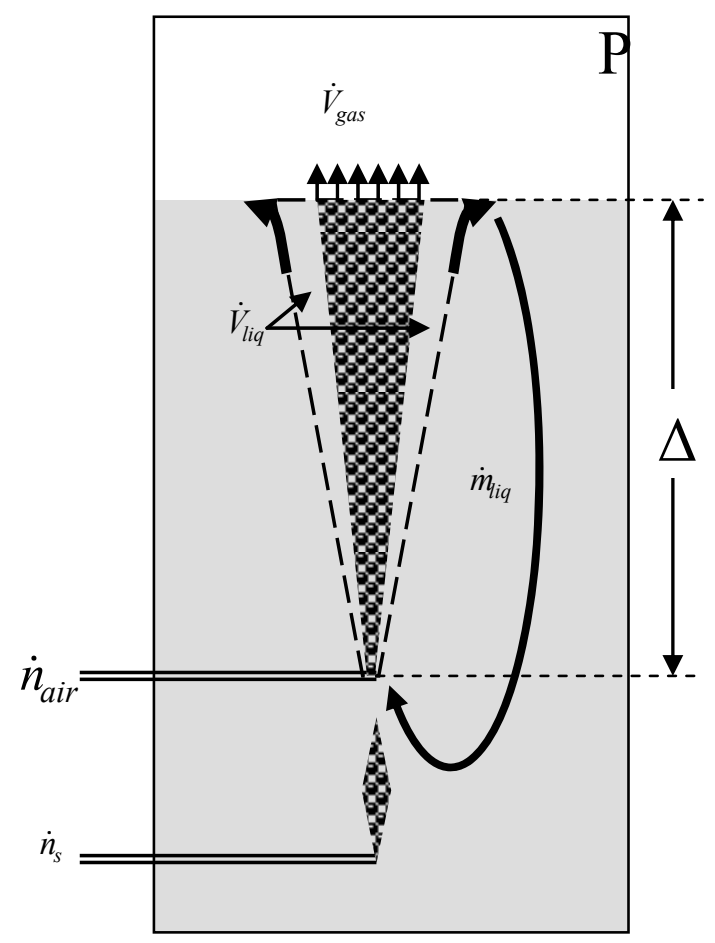

Figure A.8. Heat Transfer in Combined Bubble Regions

Although some of the enthalpy of the steam injected is rejected via heat transfer through the boundary of the tank, much of it is required to compensate for the enthalpy of water vaporized in the sparge regions. To approximate the temperature difference caused by the combination of steam injection and sparging, equate the enthalpy flow associated with these:

$$
\dot{Q}_{s}=\dot{Q}_{\text {air }}
$$

where $\dot{Q}$ is enthalpy flow, $s$ denotes injected steam, and air denotes air injected to form the sparge regions. The rate of enthalpy added due to condensation of the injected steam is

$$
\dot{Q}_{s}=\dot{n}_{s} M_{w} \Delta H^{v a p}
$$

where $\dot{n}$ is a molar flow rate, $M_{w}$ is the molecular weight of water, and $\Delta H^{v a p}$ is the enthalpy per mass of water vaporized, neglecting the small specific heat of air. The rate of enthalpy lost due to sparging is the product of the rate of vaporization of water and the enthalpy of vaporization:

$$
\dot{Q}_{a}=\dot{n}_{\text {vap }} M_{w} \Delta H^{v a p}=\frac{y_{w}}{y_{\text {air }}} \dot{n}_{\text {air }} M_{w} \Delta H^{v a p}=\frac{P_{\text {sat }}}{P-P_{\text {sat }}} \dot{n}_{\text {air }} M_{w} \Delta H^{v a p}
$$


where $P$ is the pressure at the top of the sparge region, $P_{\text {sat }}$ is the vapor pressure of water at the top of the region, and $y$ denotes a mole fraction in the sparge gas. Equating these,

$$
\dot{Q}_{s}=\dot{n}_{s} M_{w} \Delta H^{v a p}=\dot{Q}_{a}=\frac{P_{\text {sat }}}{P-P_{\text {sat }}} \dot{n}_{\text {air }} M_{w} \Delta H^{v a p}
$$

Therefore,

$$
\frac{\dot{n}_{\text {air }}}{\dot{n}_{s}}=\frac{P-P_{\text {sat }}}{P_{\text {sat }}}
$$

Sparging induces an upward mass flow of slurry due to the force imbalance imposed by the reduced average density of the fluid caused by bubbles in the sparge region. Assume we can define an effective mass flow of liquid $\dot{m}_{L}$ that provides the heat lost to vaporization during sparging. Equating the heat injected as steam with the temperature difference from the point of injection to the top of the sparge region,

$$
\dot{Q}_{s}=\dot{m}_{L} C_{p} \Delta T=\dot{n}_{s} M_{w} \Delta H^{v a p}
$$

where $\dot{m}_{L}$ is the effective mass flow rate of liquid induced by the sparging, $C_{p}$ is the specific heat, and $\Delta T$ is the temperature drop from the point of steam injection to the top of the sparge region.

Invoke the conservation of momentum to estimate the liquid velocity resulting from the void fraction in the bubble column. The hydrostatic upward force imbalance (left side of equation) is matched by the divergence of the upward liquid momentum (right side of equation) as follows.

$$
\rho_{L} g \varepsilon A(z)=\frac{d}{d z}\left[\dot{m}_{L}(z) u_{L}(z)\right]
$$

where $A(z)=$ effective flow cross-section area

$g=$ acceleration of gravity

$\varepsilon=$ void fraction in the bubble column

$u_{L}=$ upward velocity of the liquid in the bubble column.

Implicit is the assumption that profiles in the velocity and void fraction can be approximated by average values associated with the area $A(z)$. Because the gas is expanding and bubbles are continuously breaking up, assume that the void fraction is at a maximum value while the area $A(z)$ increases due to the increasing gas volumetric flow rate. The gas volumetric flow rate is related to the rise velocity by

$$
\begin{gathered}
\dot{V}_{\text {gas }}=u_{\text {rise }} A(z) \varepsilon \quad \therefore A(z) \varepsilon=\frac{\dot{V}_{\text {gas }}}{u_{\text {rise }}} \\
\rho_{L} g \frac{\dot{V}_{\text {gas }}}{u_{\text {rise }}}=\frac{d}{d z}\left[\dot{m}_{L}(z) u_{L}(z)\right]
\end{gathered}
$$


Integrate from the bottom $\left(\mathrm{z}_{0}\right)$ to the top $\left(\mathrm{z}_{1}\right)$ of the bubble column

$$
\int_{z_{0}}^{z_{1}} \rho_{L} g \frac{\dot{V}_{\text {gas }}}{u_{\text {rise }}} d z=\dot{m}_{L}\left(z_{1}\right) u_{L}\left(z_{1}\right)-\dot{m}_{L}\left(z_{0}\right) u_{L}\left(z_{0}\right)
$$

Neglect the mass flow at bottom of column compared to top. Treat the rise velocity as though it is approximately constant. Then

$$
\dot{m}_{L}\left(z_{1}\right) u_{L}\left(z_{1}\right)=\frac{\rho_{L} g}{u_{\text {rise }}} \int_{z_{0}}^{z_{1}} \dot{V}_{g a s} d z
$$

The gas flow rate is a function of local pressure:

$$
\dot{V}_{g a s}=\dot{n}_{a i r} \frac{R T}{P-P_{\text {sat }}}
$$

Substituting and integrating over the height of the bubble column,

$$
\begin{aligned}
& \dot{m}_{L}\left(z_{\text {top }}\right) u_{L}\left(z_{\text {top }}\right)=\frac{\rho_{L} g \dot{n}_{\text {air }}}{u_{\text {rise }}} \int_{z_{0}}^{z_{1}} \frac{R T}{P-P_{\text {sat }}} d z=\frac{\rho_{L} g \dot{n}_{\text {air }}}{u_{\text {rise }}} \int_{z_{\text {botom }}}^{z_{\text {top }}} \frac{R T}{P_{\text {top }}-P_{\text {sat }}-\rho_{L} g\left(z-z_{\text {top }}\right)} d z \\
& =-\frac{R T \dot{n}_{\text {air }}}{u_{\text {rise }}} \int_{z_{\text {bottom }}}^{z_{\text {top }}} \frac{d\left[P_{\text {top }}-P_{\text {sat }}-\rho_{L} g\left(z-z_{\text {top }}\right)\right]}{P_{\text {top }}-P_{\text {sat }}-\rho_{L} g\left(z-z_{\text {top }}\right)}=\frac{R T \dot{n}_{\text {air }}}{u_{\text {rise }}} \ln \frac{P_{\text {top }}-P_{\text {sat }}+\rho_{L} g\left(z_{\text {top }}-z_{\text {bottom }}\right)}{P_{\text {top }}-P_{\text {sat }}}
\end{aligned}
$$

Rearranging,

$$
\dot{m}_{L}\left(z_{\text {top }}\right)=\frac{R T \dot{n}_{\text {air }}}{u_{\text {rise }} u_{L}\left(z_{\text {top }}\right)} \ln \left(1+\frac{\rho_{L} g\left(z_{\text {top }}-z_{\text {bottom }}\right)}{P_{\text {top }}-P_{\text {sat }}}\right)
$$

The liquid mass flow rate can be written in terms of the flow cross-section and the void fraction as

$$
\dot{m}_{L}\left(z_{\text {top }}\right)=\rho_{L}(1-\varepsilon) u_{L} A=\frac{\rho_{L}(1-\varepsilon) u_{L}}{u_{r i s e} \varepsilon} \dot{V}_{\text {gas }}=\frac{\rho_{L}(1-\varepsilon) u_{L}}{u_{\text {rise }} \varepsilon} \dot{n}_{\text {air }} \frac{R T}{P_{\text {top }}-P_{\text {sat }}}
$$

Solving for the velocity of the liquid,

$$
u_{L}=\sqrt{\frac{\varepsilon\left(P_{\text {top }}-P_{\text {sat }}\right)}{\rho_{L}(1-\varepsilon)} \ln \left(1+\frac{\rho_{L} g\left(z_{\text {top }}-z_{\text {bottom }}\right)}{P_{\text {top }}-P_{\text {sat }}}\right)}
$$

The rise velocity of the gas is the terminal velocity of bubbles plus the liquid velocity

$$
u_{\text {rise }}=u_{g}+u_{L}
$$


The recirculating liquid mass-flow rate is related to the gas volumetric flow rate at the top of the bubble column by

$$
\dot{m}_{L}=\rho_{L} u_{L} A=\rho_{L} u_{L} \frac{\dot{V}_{g a s}}{u_{\text {rise }} \varepsilon}=\rho_{L} \frac{u_{L}}{\left(u_{g}+u_{L}\right) \varepsilon} \dot{V}_{g a s}
$$

Having treated the gas terminal velocity $u_{g}$, liquid velocity $u_{L}$, and void fraction $\varepsilon$ as constants to within the error associated with those approximations, we find the mass flow rate of the recirculating liquid to be proportional to the volumetric gas flow rate at the top of the bubble column. That is, we have

$$
\dot{m}_{L}=\rho_{L} \kappa \dot{V}_{\text {gas }} \quad \kappa=\frac{u_{L}}{\left(u_{g}+u_{L}\right) \varepsilon}
$$

This approach is consistent with the apparent proportionality between the size of the Zone of Influence and the Region of Bubbles found from sparging tests. Given that $\varepsilon \leq 1$ and expecting $u_{L}$ to be on the order of $u_{g}$, a reasonable guess for $\kappa$ is unity. The volumetric gas flow rate is

$$
\dot{V}_{\text {gas }}=\dot{V}_{\text {air }} \frac{P}{P-P_{\text {sat }}}=\dot{n}_{\text {air }} \frac{R T}{P} \frac{P}{P-P_{\text {sat }}}=\dot{n}_{\text {air }} \frac{R T}{P-P_{\text {sat }}}
$$

Substituting,

$$
\dot{m}_{w}=\rho \kappa \dot{n}_{\text {air }} \frac{R T}{P-P_{\text {sat }}}=\rho \kappa \dot{n}_{s} \frac{R T}{P_{\text {sat }}}
$$

Substituting into the heat balance over the induced liquid mass flow and solving for the temperature difference for operating conditions near $100^{\circ} \mathrm{C}$,

$$
\Delta T=\frac{1}{\kappa} \frac{M_{w} P_{\text {sat }} \Delta H^{v a p}}{\rho R T C_{p}} \sim \frac{1}{\kappa} \frac{(0.018 \mathrm{~kg} / \mathrm{mol})\left(10^{5} \mathrm{~J} / \mathrm{m}^{3}\right)(2,260,000 \mathrm{~J} / \mathrm{kg})}{\left(1000 \mathrm{~kg} / \mathrm{m}^{3}\right)(8.314 \mathrm{~J} / \mathrm{mol}-\mathrm{K})(373 \mathrm{~K})(4186 \mathrm{~J} / \mathrm{kg}-\mathrm{K})}=\frac{0.313 \mathrm{~K}}{\kappa} \text { (Eq. A.244) }
$$

That is, the temperature difference associated with the heat flow from the point of steam injection to the top of the sparge region is on the order of $0.3 \mathrm{~K}$ for $\kappa \sim 1$.

Although several assumptions underlie the approximation, a robust result is that the magnitude of the temperature difference is small. 



\section{Appendix B}

\section{Scaling Sparger Operations}





\section{Appendix B}

\section{Scaling Sparger Operations}

Air spargers are deployed in the Ultra-Filtration Process (UFP)-2 vessels to supplement pulse-jet mixers (PJMs), creating a "hybrid" mixing system. Additionally, steam rings in the UFP-1 and UFP-2 vessels are to be purged with air when steam is not flowing to prevent the back-flow of slurry. Because the steam rings contain numerous orifices and are located deep within the slurry-filled vessels, they too act as air spargers and contribute mixing energy when air is flowing. The following sections address the design of the dedicated air spargers in the UFP-2 vessels and the scaling of steam ring purge air flow rates in both UFP-1 and UFP-2. The latter is based on the general design principles established in the first section.

\section{B.1 Air Sparger Design}

The primary mixing work of air spargers is the result of the net vertical rise of sparge bubbles through relatively dense slurry and the resulting localized entrainment and bulk motion of slurry fluid. The sparger "mixing effectiveness" is a product of the "intensity of mixing" and the distribution of this "mixing energy" across the slurry contained in the vessel. The derivation of expressions for sparge bubble mixing power, calculations guiding the distribution of spargers in the vessels, and a specific example describing the scaling of sparger design and operation from the WTP- to PEP-scale UFP-2 vessels is provided in this section.

\section{B.1.1 Sparge Bubble Mixing Power}

The pressure-volume expansion work of a bubble rising through a differential pressure change $\mathrm{d} P$ resulting from a differential elevation change $\mathrm{d} z$ in a slurry of constant density ${ }^{1} \rho$ is

$$
d W_{b}(z)=v_{b, z} d P=v_{b, z} \rho g d z
$$

where $g$ is the acceleration of gravity and $v_{b, z}$ is the elevation-dependent bubble volume. The work accomplished in some finite elevation change from an initial elevation $z_{0}$, typically $z_{0}=0$ at the sparge tip nozzle, to an arbitrary elevation $z$ is found by integrating Equation (B.1)

$$
W_{b, z}=\int_{z_{0}}^{z} v_{b, z} \rho g d z
$$

\footnotetext{
1 The constant density assumption neglects relatively small differences due to temperature variation and is limited to cases where steam addition (as water) is approximately equal to the amount vaporized and stripped by sparge air.
} 
The power associated with the rise of a steady stream of bubbles is the work per unit time, which is simply the work of a bubble multiplied by the bubble frequency. Neglecting bubble coalescence and breakup, the bubble frequency $f_{b}$ is the flow rate of a sparge air stream $Q$ divided by the individual bubble volume

$$
f_{b}=\frac{Q_{0}}{v_{b 0}}=\frac{Q_{z}}{v_{b, z}}
$$

Implicit in Equation (B.3) is that the elevation dependence of the volume of a single bubble and the bulk sparge gas volumetric flow rate (including water vapor) are directly proportional. After passing through slurry, sparge bubbles will contain air and water vapor. Since both are gaseous, the general phrase sparge "gas" is used here when referring to bubbles containing air and water vapor. Sparge "air" refers explicitly to the essentially water vapor-free air delivered through the sparge tubes.

Substituting Equation (B.3) in (B.2) and dividing by the volume of slurry affected, $V_{s l}$, gives the volume specific integrated bubble power, $P_{b V}$

$$
P_{b V}=\frac{\int_{z_{0}}^{z} Q_{z} \rho g d z}{V_{s l, z}}=\frac{\int_{z_{0}}^{z} Q_{z} \rho g d z}{A_{s l}\left(z-z_{0}\right)}=\frac{\int_{z_{0}}^{z} U_{z}^{*} \rho g d z}{\Delta z}=\rho g \bar{U}^{*}
$$

The rightmost integral form of Equation (B.4) shows $P_{b V}$ in terms of the elevation-dependent sparge gas superficial velocity $U_{z}^{*}$

$$
U_{z}^{*}=\frac{Q_{z}}{A_{s l}}
$$

where $A_{s l}$ is the cross-sectional area of the slurry in the vessel influenced by the sparge gas. It is assumed that the PJM-free cross section projected normal to the vertical sparge tubes is the area "affected" by the sparge gas. ${ }^{1}$ Equation (B.4) indicates that the local sparge gas flow rate and superficial velocity, $Q_{z}$ and $U_{z}^{*}$, are representative of, and proportional to, the differential (or incremental) bubble power per unit volume.

Equation (B.4) also shows that for constant slurry density, the integrated bubble power per unit volume of slurry is directly related to the integrated average sparge gas superficial velocity, $\bar{U}^{*}$. To help assess sparged gas mixing effects, it is of interest to look at both the integrated forms $\left(P_{b V}\right.$ or $\left.\bar{U}^{*}\right)$ and local distribution $\left(Q_{z}\right.$ or $\left.U_{z}^{*}\right)$ of bubble mixing power. However, the elevation-dependent superficial velocity is most representative of the local slurry velocity and "mixing intensity." It is the metric used in this analysis.

\footnotetext{
${ }^{1}$ Other than the PJM cluster, no correction is made for internal structures (e.g., tubing and hardware) in the vessel that would effectively reduce the free cross-sectional area. The correction is assumed to be small, and it would apply uniformly to all cases.
} 
Equation (B.4) is applied separately to the annular and central slurry volumes of the UFP-2 vessel, utilizing the specified deep and shallow sparge air feed flow rates, respectively. An overall average superficial velocity at elevation $\mathrm{z}$ is determined from the total gas flow rate resulting from operation of all spargers divided by the total vessel cross-sectional area affected (i.e., area normalization). At elevations lower than the shallow sparger nozzle tips, the normalized $U_{z}^{*}$ (or $\left.Q_{z}\right)$ values are equal to those for the deep spargers alone.

The elevation $(z)$ dependence of the volume of an individual bubble is a function of temperature change, the change in moles of gas and vapor species (e.g., due to water vapor addition), and the change in hydrostatic pressure in the vicinity of the bubble. For a bubble of initial volume $v_{b 0}$ and other initial conditions identified by the subscript 0 , the z-dependent bubble volume is given by

$$
\frac{v_{b, z}}{v_{b 0}}=\left(\frac{T_{b, z}}{T_{b 0}}\right)\left[\left(\frac{P_{z}}{P_{z}-\eta_{z} P_{s a t, z}}\right)\left(\frac{P_{0}-\eta_{0} P_{s a t, 0}}{P_{0}}\right)\right]\left(\frac{P_{0}}{P_{z}}\right)=\frac{Q_{z}}{Q_{0}}
$$

where the first term is the thermal expansion factor ( $T_{b}$ is the bubble temperature), the middle term accounts for water vaporization (or condensation), and the last term is the pressure expansion (or compression) factor. The absolute pressure at elevation $z$ above the sparger nozzle and depth $h$ from the slurry surface, which is the submergence of the sparger nozzle less the current elevation, $H-z$, is

$$
P_{z}=P_{a}+\rho g(H-z)
$$

where $P_{a}$ is the ambient pressure in the headspace of the vessel.

The middle term of Equation (B.6) is a function of both the absolute pressure at elevation and the temperature-dependent saturation partial pressure of water vapor, $P_{\text {sat }}$, for the slurry composition surrounding the bubble. The saturation fraction $\eta$ can be a function of $z$, and is unity when air bubbles are in mass equilibrium with the slurry (i.e., saturated with water vapor) and is zero for dry air. In the analysis of hot sparging described below, the sparge bubbles are assumed to saturate nearly instantaneously upon departure from the sparge tube $(\eta=1) .{ }^{1}$ If the bubble temperature is constant, for example in the case where the sparge gas is pre-heated during transit through the sparge tubes and sufficient heat is provided to maintain nearly constant $T_{b}$ throughout the bubble rise, Equation (B.6) reduces to

$$
\frac{v_{b, z}}{v_{b 0}}=\left(\frac{P_{0}-P_{\text {sat }}}{P_{z}-P_{\text {sat }}}\right)=\left[\frac{P_{a}+\rho g\left(H-z_{0}\right)-P_{\text {sat }}}{P_{a}+\rho g(H-z)-P_{\text {sat }}}\right]=\frac{Q_{z}}{Q_{0}}
$$

\footnotetext{
${ }^{1}$ The assertion of rapid attainment of water-vapor equilibrium in the sparge-gas bubbles is based in part on the work of Smith (2006), who estimated reaching near equilibrium water concentration in stagnant small (e.g., $<1 \mathrm{~cm})$ bubbles in a few seconds or less. Convection within and around rising bubbles would increase the rate of watervapor mass transfer. However, depending on sparge air-flow rates and/or slurry physical properties, sparge bubbles may be relatively large upon separation from the nozzle tip $($ e.g., $10 \mathrm{~cm}$ ), which would result in an increased time to saturation.

Smith JM. 2006. “Large Multiphase Reactors, Some Open Questions.” Trans IChemE, Part A, 84(A4):265-271.
} 
As $P_{\text {sat }}$ approaches the absolute pressure at elevation z, bubble volume (and flow rate) can become quite large as the denominator of Equation (B.8) becomes small. Since $P_{\text {sat }}$ increases with temperature, sparging hot can lead to dramatic increases in sparge gas flow rates and commensurate enhancement in (mixing intensity), particularly near the slurry surface. $P_{\text {sat }}$ is also a function of slurry composition, generally decreasing with increasing concentration of ionic species (e.g., sodium hydroxide and salts).

One sparger scaling approach is to match the elevation-specific gas superficial velocity achieved under design basis operating conditions. Of interest is the flow rate $Q_{\text {std }}$ of dry sparge air under standard conditions $\left(P_{0}=P_{a}=1 \mathrm{~atm} ; \eta P_{\text {sat }}=0 ; T_{0}=T_{b 0}=25^{\circ} \mathrm{C}\right)$ needed to provide a specified gas superficial velocity $U_{z}^{*}$ at elevation $z$ under hot operating conditions. Rearranging Equation (B.5) gives $Q_{z}$ for the cross-sectional area affected by the spargers $\left(=U^{*}{ }_{z} A_{s l}\right)$. Assuming constant slurry and bubble temperature conditions $\left(T=T_{s l}=T_{b}\right)$ and equilibrium saturation of the bubbles to the water vapor partial pressure immediately upon exiting from the sparger nozzle $(z=0)$, and substituting for $Q_{z}$ into Equation (B.6) gives

$$
Q_{s t d}=\left(\frac{T_{0}}{T_{s l}}\right)\left(\frac{P_{a}+\rho g(H-z)-P_{s a t}}{P_{a}}\right) U_{z}^{*} A_{s l}=\left(\frac{T_{0}}{T_{s l}}\right)\left(\frac{P_{z}-P_{s a t}}{P_{a}}\right) U_{z}^{*} A_{s l}
$$

The inverted form of Equation (B.9) is also useful to determine the elevation-dependence of the total sparge gas flow rate (hence, superficial gas velocity) for a given standard sparge air feed rate

$$
Q_{z}=\left(\frac{T_{s l}}{T_{0}}\right)\left(\frac{P_{a}}{P_{z}-P_{s a t}}\right) Q_{s t d}
$$

Equation (B.10) is subject to the assumptions of Equation (B.9). This equation is utilized to determine the sparge air flow rate required in the PEP UFP-2 vessel to match superficial velocity criteria of the WTP-scale vessel. It is also used to compare the local bubble mixing power in the two vessels, as shown in examples below.

\section{B.1.2 Calculations Guiding the Spatial Distribution of Spargers}

Claghorn and Waddell ${ }^{1}$ specify the WTP-scale UFP-2 "design basis" sparger operating conditions including gas flow rates to 16 spargers at three nozzle depths. Calculated sparger regions of bubbles (ROBs) and overlap of zones of influence (ZOIs) were used as a basis to layout spargers to provide adequate mixing. Rising bubbles in ROBs entrain slurry resulting in a net upward movement of slurry, while ZOIs define regions for slurry to return to depth completing a recirculation pathway (Poloski et al. 2005). ${ }^{2}$ In support of WTP vessel mixing studies, Poloski et al. (2005) evaluated mixing resulting from

${ }^{1}$ Claghorn R and P Waddell. 2007. System Description for Pulse Jet Mixers and Supplemental Mixing Subsystems. 24590-WTP-3YD-50-00003 Rev B, Bechtel National, Inc., Richland, Washington.

2 Poloski AP, ST Arm, JA Bamberger, B Barnett, R Brown, BJ Cook, CW Enderlin, MS Fountain, M Friedrich, BG Fritz, RP Mueller, F Nigl, Y Onishi, LA Schienbein, LA Snow, S Tzemos, M White, and JA Vucelick. 2005. Technical Basis for Scaling of Air Sparging Systems for Mixing in Non-Newtonian Slurries. PNNL-3541 (WTPRPT-129 Rev. 0), Battelle - Pacific Northwest Division, Richland, Washington. 
air sparging in non-Newtonian simulants at ambient temperature and obtained expressions relating sparge gas flow rate and ROB and ZOI diameters, $D_{R O B}$ and $D_{Z O I}$,

$$
D_{R O B}=11 Q^{0.34} \quad \text { and } \quad D_{Z O I}=34 Q^{0.34}
$$

where $Q$ is the actual flow rate at the sparger nozzle in actual cubic feet per minute (acfm) and the diameters are in inches. Note that $D_{Z O I}$ is essentially three-times $D_{R O B}$. If spargers are distributed such that the nozzles are separated by $2 / 3 D_{Z O I}$, then ZOI circles of neighboring spargers just meet adjacent ROB circles, as projected on the slurry surface. In this way, the upward flow of bubbles and slurry in ROBs interferes minimally with the downward recirculation in ZOIs, while providing maximum coverage over the slurry surface area. This is the approach taken to establish the sparger layout and flow rates at ambient temperature in the WTP UFP-2 vessel. ${ }^{1}$ Equation (B.11) is not directly applicable to the airsparge rates in hot-slurry systems because of the thermal and water-vapor effects on bubble expansion [e.g., Equation (B.6)].

In Equation (B.11), the approximate proportionality of the ROB and ZOI diameters to the flow rate raised to the one-third power is included for convenience in the scaling development to follow.

As noted in the example to follow, the fundamental approach to scale the sparge air flow rate and bubble mixing power for different vessel sizes is to maintain a constant superficial gas velocity at a specified vertical location (e.g., the slurry surface, or the sparge nozzle depth). The superficial velocity is defined in Equation (B.5) above. For geometrically scaled vessels, the cross-sectional area varies as the square of the linear scale factor $S$. Defining $S$ as the ratio of the linear size of equipment in the WTP to the corresponding size in the PEP $(S=4.5)$, the cross-sectional areas are related by

$$
A_{s l, W T P}=A_{s l, P E P} S^{2}
$$

Combining Equations (B.5) and (B.12), the actual sparge air flow rates required to maintain constant superficial velocity at sparge nozzle depth in the scale vessels are also related by the square of the scale factor

$$
Q_{W T P}=Q_{P E P} S^{2}
$$

Substituting this result into the proportionality Equation (B.11) with the 0.34 power approximated as $1 / 3$ leads to expressions relating WTP and PEP-scale ROB diameters and ROB areas $\left(=\pi D^{2} / 4\right)$

$$
D_{R O B, P E P}=D_{R O B, W T P} S^{-2 / 3}
$$

and

$$
A_{R O B, P E P}=A_{R O B, W T P} S^{-4 / 3}
$$

\footnotetext{
${ }^{1}$ Claghorn R and P Waddell. 2007. System Description for Pulse Jet Mixers and Supplemental Mixing Subsystems.
} 24590-WTP-3YD-50-00003 Rev B, Bechtel National, Inc., Richland, Washington. 
Implicit in these equations is an assumption that ROB and ZOI regions are fully developed in the smaller scale vessel, ${ }^{1}$ even though the nozzle and stimulant depths are a factor of $S$ less. Using Equations (B.15) and (B.12), the fractional area of a ROB relative to the slurry surface area in the two vessel scales are related by

$$
\left(\frac{A_{R O B}}{A_{S l}}\right)_{P E P}=\left(\frac{A_{R O B}}{A_{S l}}\right)_{W T P} S^{2 / 3}
$$

This shows that in the case of constant sparge air superficial velocity at the sparger nozzles, the coverage of a single sparger is a factor of $S^{2 / 3}$ larger in the smaller-scale vessel. Since ROB and ZOI both vary as a function of flow rate to approximately the one-third (0.34) power [Equation (B.11)], the WTP and PEP ZOI diameters [Equation (B.14)], areas [Equation (B.15)], and fractional surface area coverage [Equation (B.16)] vary with the scale factor raised to a power in the same way as the ROB. For $S=4.5$, the calculated improvement is $\sim 2.7\left(=4.5^{2 / 3}\right)$. This suggests that fewer spargers are required in the PEP than in the WTP UFP-2 vessel.

To a first approximation, the number of spargers $n_{s p}$ at full-scale are related by the area ratio factor $S^{2 / 3}$ of Equation (B.16)

$$
n_{s p, P E P}=\frac{n_{s p, F S}}{S^{2 / 3}}=n_{s p, F S} S^{-2 / 3}
$$

However, reducing the number of spargers requires increasing the flow rate to the remaining spargers in order to satisfy the constant superficial velocity criterion, which in turn increases the ROB and ZOI diameters according to Equation (B.11). The volumetric flow rate to each of the scaled spargers would be increased by a factor of $S^{2 / 3}$ to compensate for the reduction in $n_{s p}$.

$$
Q_{P E P, 2}=Q_{P E P, 1} S^{2 / 3}
$$

where the subscripts 1 and 2 indicate initial and second estimates. The relative increase in ROB (and ZOI) diameter and area are determined by substituting Equation (B.18) into Equation (B.11)

$$
D_{R O B, P E P, 2}=D_{R O B, P E P, 1} S^{2 / 9}
$$

and

$$
A_{R O B, P E P, 2}=A_{R O B, P E P, 1} S^{4 / 9}
$$

The ROB (and ZOI) diameter and area for the revised number of spargers in the PEP are $40 \%$ $\left(4.5^{2 / 9}=1.40\right)$ and $95 \%\left(4.5^{4 / 9}=1.95\right)$ greater, respectively. This suggests further iterations in the reduction in the number of spargers, again with compensating proportional increases in the flow rate per remaining sparge tube.

${ }^{1}$ At $1.8 H_{s l} / D$, the deep spargers will be $\sim 62$-in. deep and the shallow spargers above the PJMs will only be $\sim 19$-in. deep. Therefore, the full ROB and ZOI diameters might not be attained (Poloski et al. 2005). 
Other practical factors must be considered in setting the number of spargers in the PEP vessel. The number and layout of spargers is also dictated by symmetry. In the case of UFP-2, there is a strong driver to keep the number of deep spargers in the annular portion of the tank to multiples of five, corresponding with the number of PJMs in the outer ring of the PJM cluster (five outer plus one center). Likewise, symmetry in the spargers in the center of the vessel over the top of the PJMs is desirable. The ROB and ZOI layout criteria used in the WTP vessel design basis should also be considered (i.e., minimize overlap of ZOIs and ROBs, optimally with sparger nozzles separated by $2 / 3 \mathrm{ZOI}$ ).

\section{B.1.3 Sparger Scaling in the PEP UFP-2 Vessel}

Figure B.1 is a flow diagram for the process of scaling sparger operating parameters and layout from the WTP- to PEP-scale UFP-2 vessels. Input parameters include the established "design basis" sparger flow rates and sparge tube distribution in the full-scale WTP UFP-2 vessel. After selecting an approach to scale the bubble mixing power or "mixing intensity" from WTP- to PEP-scale, the total sparge air feed flow rate required in the PEP-scale vessel is determined using calculations outlined in Section B.1.1. Finally, the number and distribution of spargers in the PEP vessel is set using guidelines described in Section B.1.2. As shown in the scaling process flow diagram, mixing intensity is the primary consideration, and sparger spatial layout is a secondary, although important, factor.

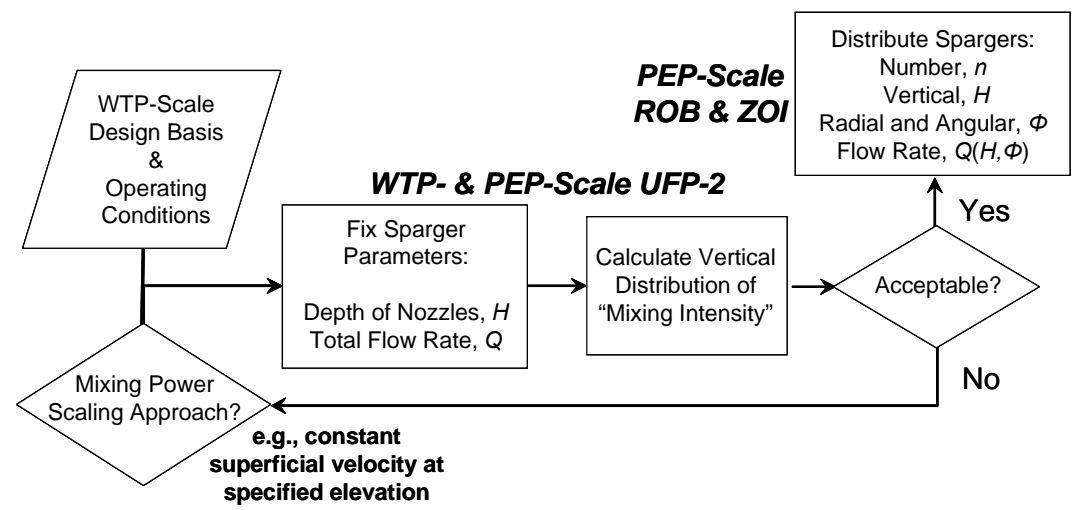

Figure B.1. Process Flow Diagram for Scaling Sparger Operation and Layout in the PEP-Scale UFP-2 Vessel

\section{B.1.3.1 UFP-2 Vessel Model and Sparger Design Basis}

Applying the equations above to estimate the sparge gas superficial velocity, the required sparge gas flow rate in the PEP vessel, and to layout the spargers in the reduced-scale UFP-2 model requires knowledge of the WTP-scale vessel configuration and operating parameters. This includes slurry fill level, sparger depths, sparger coverage areas and volumes, and slurry physical properties. The basis of values used in the PEP UFP-2 vessel sparger-scaling calculations is discussed in this section.

Figure B.2 shows two simplified, but substantively to-scale, schematics of the full-scale UFP-2 vessel. The image on the left is derived from descriptions in Claghorn and Waddell ${ }^{1}$ and UFP-2 vessel

\footnotetext{
${ }^{1}$ Claghorn R and P Waddell. 2007. System Description for Pulse Jet Mixers and Supplemental Mixing Subsystems. 24590-WTP-3YD-50-00003 Rev B, Bechtel National, Inc., Richland, Washington.
} 
drawings. ${ }^{1,2}$ Claghorn and Waddell is the source of the design-basis sparger operating conditions including $360 \mathrm{scfm}$ total sparge gas flow rate, a slurry density of $1.35 \mathrm{~kg} / \mathrm{L}$, and a slurry fill level of $2.35 H_{s l} / D$ (= slurry depth to tank bottom/vessel diameter). The $2.35 H_{s l} / D$ corresponds to filling the vessel with slurry to the elevation of the overflow port. ${ }^{3}$

In normal operation, the nominal fill level is expected to be closer to $1.8 H_{s l} / D, 4,5$ as shown in the working UFP-2 vessel model on the right in Figure B.2.
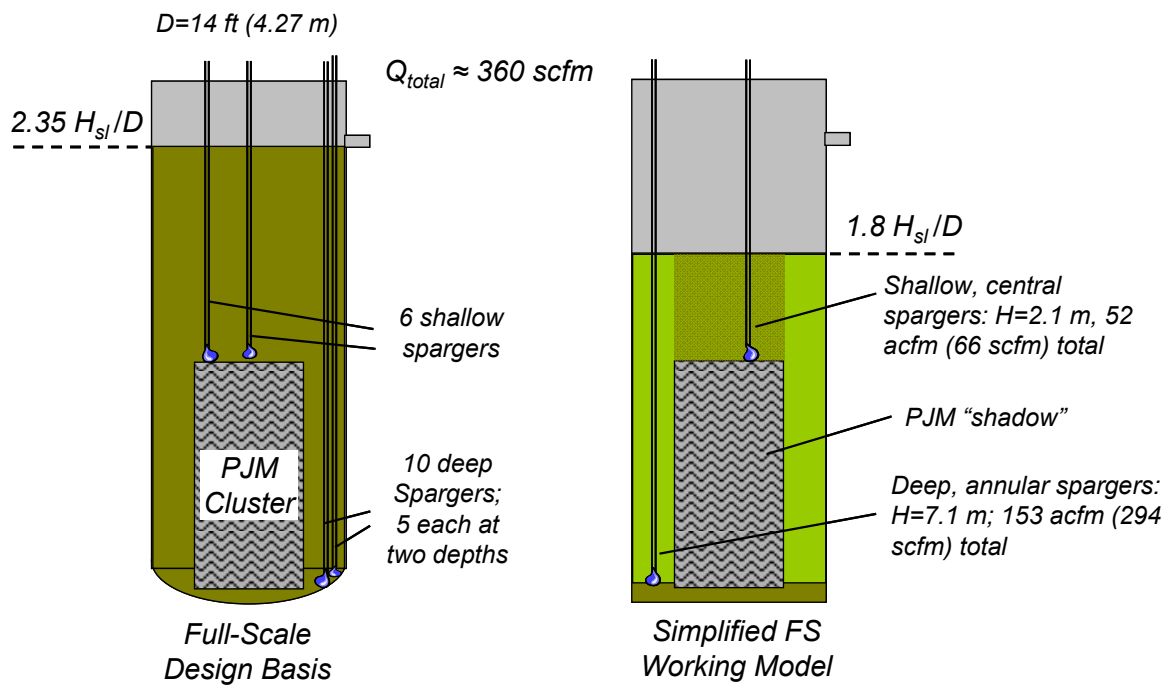

Figure B.2. WTP UFP-2 Vessel Schematics for Original Design-Basis Operating Conditions (left: $2.35 \mathrm{Hs} / \mathrm{D}$ overflow fill level and air spargers at 3 depths) and a Further Simplified Working Model (right: $1.8 \mathrm{Hsl} / \mathrm{D}$ nominal fill level with air spargers at two depths separately covering annular and central regions)

The UFP-2 "design basis" sparger operating conditions ${ }^{6}$ specify gas flow rates to 16 spargers at three nozzle depths for ambient or relatively cool operating scenarios $\left(\right.$ e.g., $\left.25^{\circ} \mathrm{C}\right)$ in which water vaporization is minimal. To simplify calculations, it is reasonable to assume that the ten deep spargers in UFP-2, five each at two elevations differing by $<4$ in., can be represented by a set of spargers at a single elevation that

${ }^{1}$ Khurana H. 2005a. Equipment Assembly Ultrafiltration Feed Vessel UFP-VSL-00002B. 24590-PTF-MV-UFP00004 Rev. 4, Bechtel National, Inc., Richland, Washington.

${ }^{2}$ Khurana H. 2005b. Layout of Internals Ultrafiltration Feed Vessel UFP-VSL-00002B. 24590-PTF-MV-UFP00018 Rev. 1, Bechtel National, Inc., Richland, Washington.

3 The data table of Figure 6.13 of Claghorn and Waddell was used to calculate the elevation to the bottom of the overflow port $(\sim 32.83 \mathrm{ft})$ and thus the $2.35 H_{s l} / D(=32.83 \mathrm{ft} / 14.0 \mathrm{ft})$. The overflow port elevation was determined from the ratio of the standard sparge air flow rate, the actual flow rate at nozzle depth, and the specified nozzle elevation above the tank bottom. This calculated overflow port elevation $(32.83 \mathrm{ft})$ is consistent with the value of $32.92 \mathrm{ft}$ shown in Figure 5 of Kufahl (2003) and the 33.0-ft result estimated from scale drawings of UFP-2 (Khurana 2005a). Kufahl MA. 2003. Vessel Sizing Calculations for UFP Ultrafiltration Vessels UFP-VSL-00002A/B. 24590-PTF-M6C-UFP-00008 Rev. C, Bechtel National, Inc., Richland, Washington.

${ }^{4}$ Kufahl MA. 2003. Vessel Sizing Calculations for UFP Ultrafiltration Vessels UFP-VSL-00002A/B. 24590-PTFM6C-UFP-00008 Rev. C, Bechtel National, Inc., Richland, Washington.

5 Stiver B. 2007. Functional Requirements for Pretreatment Engineering Platform (PEP). 24590-PTF-3YD-UFP00002, Rev 0, Bechtel National, Inc., Richland, Washington.

${ }^{6}$ Claghorn R and P Waddell. 2007. System Description for Pulse Jet Mixers and Supplemental Mixing Subsystems. 24590-WTP-3YD-50-00003 Rev B, Bechtel National, Inc., Richland, Washington. 
give the same total actual flow rate at depth in acfm for a given standard feed flow rate in scfm. This is depicted on the right side of Figure B.2, where the total sparge rate of $360 \mathrm{scfm}$ is split between a representative deep sparger (294 scfm) and a representative shallow sparger (66 scfm). The deep spargers are expected to affect the slurry volume in the annular region of the tank and the shallow spargers should mobilize slurry in the center of the tank above the PJM cluster. ${ }^{1}$

The water vapor saturation partial pressure of the slurry is a key parameter in calculation of the sparge gas superficial velocity in hot slurries. ${ }^{2}$ In this report, the waste slurry composition during hot caustic leaching in UFP-2 is modeled as sodium hydroxide solution $(\mathrm{NaOH})$. It is understood that waste slurry containing a certain $\mathrm{NaOH}$ concentration may have a $P_{\text {sat }}$ value different than that for pure $\mathrm{NaOH}$ solution at that temperature because the slurry contains a mixture of dissolved salts that also influence $P_{\text {sat }}$. However, since $\mathrm{NaOH}$ is a primary ionic component of the slurry, the pure $\mathrm{NaOH}$ model should give $P_{\text {sat }}$ values in the range expected during caustic leaching.

\section{B.1.3.2 Sparge-Gas Flow Rate and Mixing Intensity in the PEP UFP-2 Vessel}

Example calculations showing the relationship of sparge air flow rates to resulting sparge gas (air + water vapor) flow rates and mixing intensity are provided. A recommended sparge air flow rate scaling approach is also described. The recommended PEP UFP-2 vessel sparge air flow rates for use during ambient and high temperature processes are reiterated in a summary section.

\section{Sparge-Gas Flow Rate and Mixing Intensity Calculations}

Approaches for reducing the sparge air flow rate when sparging in hot slurries (e.g., during caustic leaching) while providing adequate mixing, minimizing undesired cooling, steam demand, and gas holdup (retained gas) are the subject of a letter report ${ }^{3}$. In the full-scale WTP UFP-2 vessels, the selected approach is to match the superficial gas velocity $\left(U^{*}\right)$ of the hot system to that of the $25^{\circ} \mathrm{C}$ design basis system at specific reference elevations (z): (a) $2 / 3$ of the elevation from the deep, annular sparger nozzles; and (b) $1 / 2$ of the elevation of the shallow, central spargers (which equals 0.85 of the deep sparger elevation for a vessel fill level to tank diameter ratio of $1.8 H_{s l} / D$ ). Generally, it is not possible to match the elevation-dependent superficial gas velocity profiles for hot systems and the $25^{\circ} \mathrm{C}$ design basis system throughout the sparged region, but calculations indicate that reasonable compromises are attained

\footnotetext{
1 See the WTP UFP-2 vessel drawings for the PJM dimensions and layout used in the following calculations (Khurana H. 2005a. Equipment Assembly Ultrafiltration Feed Vessel UFP-VSL-00002B. 24590-PTF-MV-UFP00004 Rev. 4, Bechtel National, Inc., Richland, Washington; Khurana, H. 2005b. Layout of Internals Ultrafiltration Feed Vessel UFP-VSL-00002B. 24590-PTF-MV-UFP-00018 Rev. 1, Bechtel National, Inc., Richland, Washington; Khurana H. 2005c. Sections and Details Ultrafiltration Feed Vessel UFP-VSL-00002A \& B. 24590PTF-MV-UFP-00017 Rev. 1, Bechtel National, Inc., Richland, Washington.). The area over the PJMs covered by the shallow spargers is the PJM circle $(6 \mathrm{ft}, 2 \mathrm{in}$. diameter $)+\sim 60 \% \times 5 \mathrm{PJMs} \times \sim 2 \mathrm{ft}, 10 \mathrm{in}$. OD each $(2 \mathrm{ft}, 8 \mathrm{in}$. ID + $1 \mathrm{in}$. wall estimated). This is the approximate area covered by the PJM shroud plus the protrusion of the five outer PJMs beyond the shroud circumference (= the PJM centerline). In this way, it is estimated that 68.3 and $31.7 \%$ of the vessel sectional area is covered by the deep and shallow spargers, respectively. Considering these area ratios and the slurry depth from the nozzle tips to the surface for a $1.8 H_{s l} / D$ fill level, $88 \%$ of the slurry volume affected by the spargers is in the annular region, and $12 \%$ is in the central region above the PJMs.

${ }^{2}$ In these calculations, the water content of feed sparge air is assumed to be negligible (compared to the saturation partial pressure of water in hot waste slurries); the feed air is considered "dry."

3 Rassat SD. August 2007. A Scaling Approach for Full-Scale Sparger Operations in the UFP-2 Vessel. WTP-RPT-162, Pacific Northwest National Laboratory, Richland, Washington.
} 
using these scaling criteria. Since PJMs will provide significant mixing effectiveness in the lower third to half of the UFP-2 vessel, sparger performance is of greatest importance above this region (provided that spargers also circulate fluid to and from the PJM cavern).

Such an approach is used here to specify sparge air feed flow rates in the PEP UFP-2 vessel at ambient temperature and at the proposed caustic leaching temperature, $100^{\circ} \mathrm{C} .{ }^{1}$ Figure B. 3 shows calculated results at the design basis temperature $\left(25^{\circ} \mathrm{C}\right)$ for $1.35-\mathrm{kg} / \mathrm{L}$ slurry filled to a level of $1.8 H_{s l} / D$.
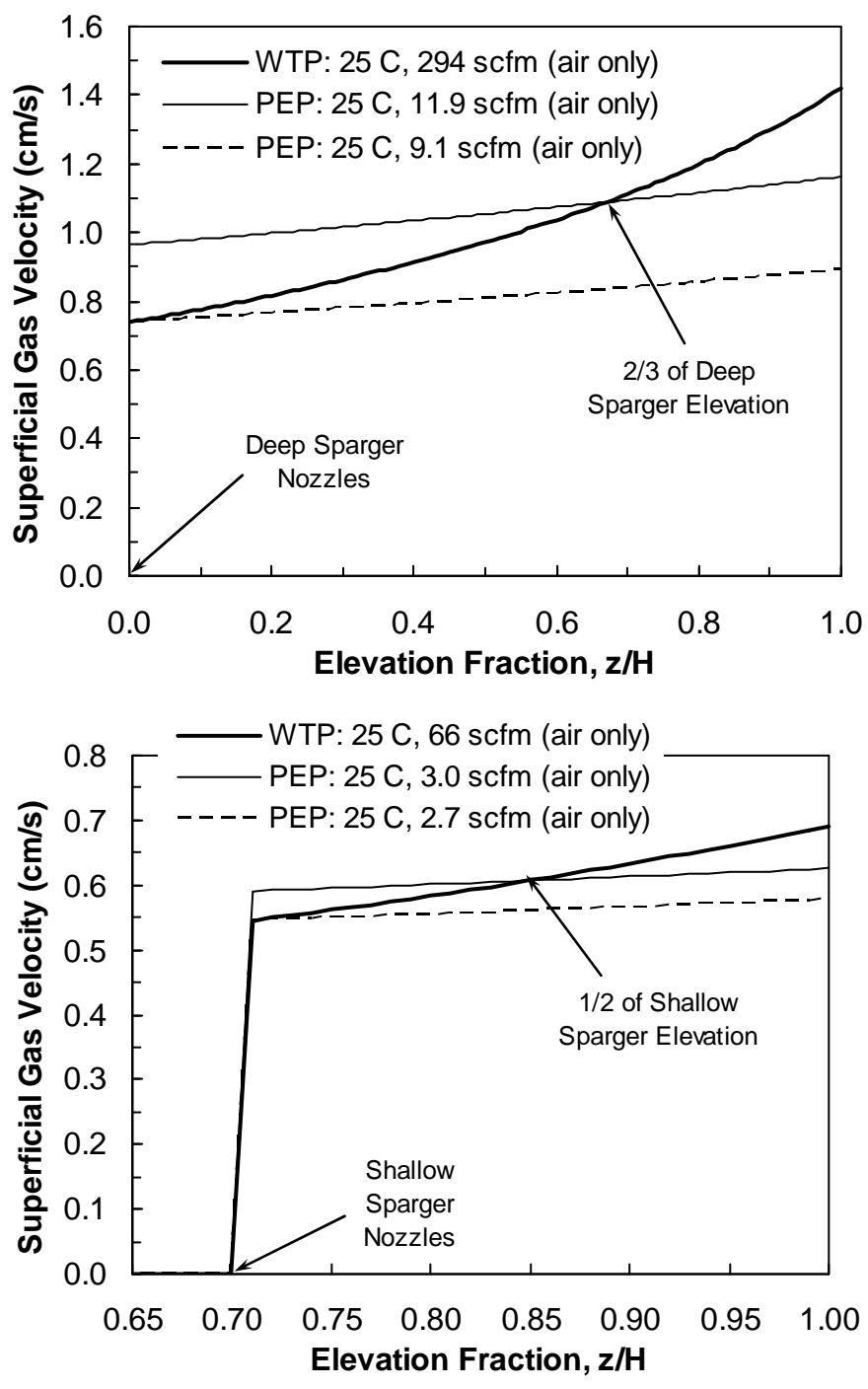

Figure B.3. Superficial Sparge Gas Velocity as a Function of Sparge Air Flow Rate and the Fractional Elevation above the Deep Sparger Nozzle Depth z/H in the WTP and PEP UFP-2 Vessels at $25^{\circ} \mathrm{C}$ : Upper - in the Annular, Deep Sparger Region; Lower - in the Central, Shallow Sparger Region (assuming the sparge bubbles remain "dry" = "air only;" 1.8-Hsl/D fill level; $1.35-\mathrm{kg} / \mathrm{L}$ slurry density. Resolution in plotted $\mathrm{z} / \mathrm{H}$ values results in sloping of the "step function" in $\mathrm{U}^{*}$ near the shallow sparger nozzle elevation.)

\footnotetext{
1 Olson JW and EJ Slaathaug. 2007. Design Evaluations Supporting Resolution of External Flowsheet Review Team (EFRT) Issue M12 and Plant Capacity Issues Related to Ultrafiltration and Leaching. 24590-WTP-RPTENG-06-014 Rev. 0, Bechtel National, Inc., Richland, Washington.
} 
It shows separately for the annular deep sparger region and the central shallow sparger region the sparge gas superficial velocity as a function of the fractional elevation $(z / H)$ above the deep sparger nozzle depth $(H)$. A step-function in $U^{*}$ in the shallow sparger region occurs at just over $0.70 z / H$ corresponding to the elevation at which air is introduced. The full-scale WTP design basis (360 scfm total $=294 \mathrm{scfm}$ deep + $66 \mathrm{scfm}$ shallow) is compared to the PEP-scale at reduced total flow rates chosen to match the design basis $U^{*}$ values according to the following criteria:

- Matching $U^{*}$ at Intermediate Depths - $14.8 \mathrm{scfm}$ total sparge air (11.9 scfm to the deep annular spargers $+3.0 \mathrm{scfm}$ to the shallow central spargers) is needed to match the design basis $U^{*}$ value at the proposed WTP UFP-2 vessel reference elevations $(1.09 \mathrm{~cm} / \mathrm{s}$ at $0.67 \mathrm{z} / H$ for the deep spargers and $0.61 \mathrm{~cm} / \mathrm{s}$ at $0.85 \mathrm{z} / \mathrm{H}$ for the shallow spargers).

- Matching $U^{*}$ at Nozzle Depth $-11.8 \mathrm{scfm}$ total sparge air (=9.1 scfm to the deep annular spargers + $2.7 \mathrm{scfm}$ to the shallow central spargers) is needed to match $U^{*}$ at the nozzle elevation of the WTP design basis system $(0.74 \mathrm{~cm} / \mathrm{s}$ for the deep spargers and $0.54 \mathrm{~cm} / \mathrm{s}$ for the shallow spargers $)$.

Matching $U^{*}$ at intermediate depth $(0.67 z / H$ for the deep spargers and $0.85 z / H$ for the shallow spargers) is a compromise in which the calculated superficial velocity of the PEP system is greater than the WTP system below the reference elevation and lower above it. On average, the bubble mixing power in the two systems is about equal using this scaling approach (14.8 scfm total in the PEP). Matching $U^{*}$ at nozzle depth leads to relatively less "mixing intensity," as determined by the $U^{*}$ profile, throughout the slurry depth in the PEP vessel. Operating the PEP system with this reduced sparge air flow rate (11.8 scfm total) for low temperature processes is conservative from a bubble mixing power perspective. However, the blend time would still be less than that for the WTP system with either of these PEP sparge rates (see Appendix A, Section A.2.6.1).

A third approach (not shown in Figure B.3) is to match $U^{*}$ of the WTP design basis at the slurry surface. In this case, PEP sparge rates are calculated by simple area scaling of the WTP vessel standard sparge air flow rate using a scale factor $S$ of 4.5 (i.e., $17.8 \mathrm{scfm}$ total $=360 \mathrm{scfm}$ total $/ 4.5^{\wedge} 2$ ). This reduced sparge rate $(17.8 \mathrm{scfm}$ total $=14.5 \mathrm{scfm}$ deep $+3.3 \mathrm{scfm}$ shallow $)$ results in a superficial velocity profile greater than the WTP-scale design basis throughout the PEP vessel and is, therefore, not conservative.

As shown in Figure B.4, applying the full-scale superficial velocity matching criteria to the PEP under nominal operating conditions of $100^{\circ} \mathrm{C}$ in $5.0-\mathrm{M} \mathrm{NaOH}$ results in a surprisingly good match to the $25^{\circ} \mathrm{C}$ WTP design basis $U^{*}$ profile. (The short, dashed line is almost indistinguishable from the heavy solid line representing the design basis.) The agreement is much better than that obtained in the WTPscale hot reference case. The PEP profile is flatter because of the much more limited change in pressure from the sparge nozzles to the surface in the small-scale system (factor of $\sim 1.2$ maximum instead of $\sim 1.9$ at full scale). However, compared to Figure B.3, the PEP $U^{*}$ profile in hot slurry of Figure B.4 increases more with elevation than in the $25^{\circ} \mathrm{C}$ PEP cases. This is due to the increasing water vapor enhancement of sparge gas flow rate near the surface, where $P_{\text {sat }}$ approaches the ambient headspace pressure [e.g., Equation (B.10)].

Other methods to scale bubble mixing power in the hot PEP are possible. For example, a total sparge air flow rate of $5.1 \mathrm{scfm}(=4.4 \mathrm{scfm}$ deep $+0.75 \mathrm{scfm}$ shallow $)$ is needed in the PEP to match $U^{*}$ at the slurry surface of the hot WTP-scale system at reduced sparge rate $\left(104 \mathrm{scfm}\right.$ total air in; $U^{*}$ at the surface is $2.84 \mathrm{~cm} / \mathrm{s}$ for the deep spargers and $1.05 \mathrm{~cm} / \mathrm{s}$ for the shallow spargers). These equivalent PEP sparge 

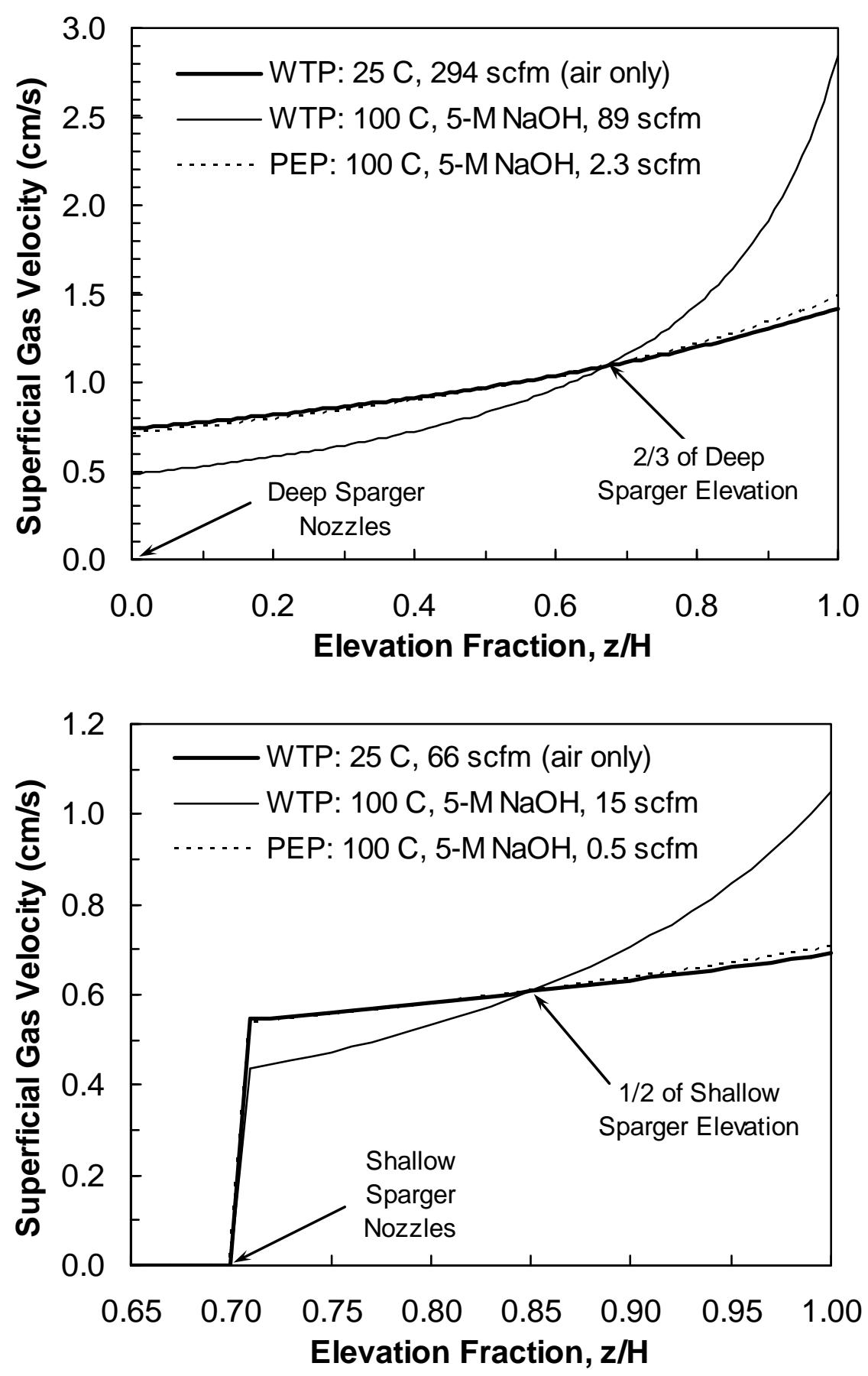

Figure B.4. Superficial Sparge Gas Velocity as a Function of the Fractional Elevation above the Deep Sparger Nozzle Depth $z / H$ for Design Basis and Hot Operating Conditions in WTP and PEP UFP-2 Vessels: Upper Figure - in the Annular, Deep Sparger Region; Lower Figure- in the Central, Shallow Sparger Region $\left(100^{\circ} \mathrm{C}, 5.0-\mathrm{M} \mathrm{NaOH}\right.$ gives $616-\mathrm{mm} \mathrm{Hg} P_{\text {sat }}$, assume the sparge gas is saturated with water vapor at the slurry $T$, except that it is "dry" = "air only" at $25^{\circ} \mathrm{C} ; 1.8-H_{s l} / D$ fill level; $1.35-\mathrm{kg} / \mathrm{L}$ slurry density. Resolution in plotted $z / H$ values results in sloping of the "step function" in $U^{*}$ near the shallow sparger nozzle elevation.) 
rates are calculated by simple area scaling of the WTP- and PEP-scale vessels using a scale factor $S$ of 4.5 (i.e., $5.14 \mathrm{scfm}$ total $=104 \mathrm{scfm}$ total $/ 4.5^{\wedge} 2$ ). Another approach is to match $U^{*}$ at the sparger nozzle depth of the hot full-scale system at reduced sparge rate (104 scfm total). This results in a superficial velocity profile lower than the WTP design basis throughout the vessel and is not considered further.

The analyses described above pertain to "normal" sparger operations. If the waste fill level is less than $1.4 H_{s l} / D$, an "idle" air sparge rate of $\sim 2 \mathrm{scfm}$ per tube or $32 \mathrm{scfm}$ total is specified for the WTP UFP-2 vessels. ${ }^{1}$ Scaling by the vessel area ratio, the corresponding idle sparge-air-flow rate in the PEP is $1.6 \mathrm{scfm}$ total (=32 scfm total $\left./ 4.5^{\wedge} 2\right)$. This is equivalent to matching the idle sparge-gas superficial velocity at the surface in the WTP and PEP vessels, and it results in a maximum (non-conservative) idle sparge rate in the PEP vessel. Alternatively, a nominal idle sparge air flow rate in the PEP can be set based on matching $U^{*}$ at an intermediate elevation as described above. The equivalent idle sparge rate in the PEP is $1.3 \mathrm{scfm}$ (= 32-scfm WTP idle sparge air * [14.8-scfm PEP normal sparge air / 360-scfm WTP normal sparge air]).

Claghorn and Waddell ${ }^{1}$ did not specify a split of the idle sparge air between deep and shallow spargers but rather gave a constant per-tube value. If the nominal idle sparge air were to be split proportional to the flow rates in normal WTP and PEP operations, 1.06-scfm air would go to the deep spargers and $0.26 \mathrm{scfm}$ to the shallow spargers in the PEP. A per-tube idle sparge rate in the PEP can only be determined after the total number of sparge tubes in the PEP is specified. Assuming six sparge tubes (see Section B.3.3), the per tube idle air sparge rate is $0.22 \mathrm{scfm}$.

\section{Sparge Air Flow Rate Summary}

In summary, the following PEP UFP-2 vessel sparge air flow rates are recommended to produce nominal bubble mixing power equal to that in the WTP vessel for the same conditions:

- Normal Operation at $25^{\circ} \mathrm{C}-14.8 \mathrm{scfm}$ total sparge air $=11.9 \mathrm{scfm}$ to the deep annular spargers + $3.0 \mathrm{scfm}$ to the shallow central spargers; independent of slurry composition (i.e., negligible water vaporization assumed)

- Normal Operation at $100^{\circ} \mathrm{C}$ in 5.0-M NaOH Slurry-2.8 scfm total sparge air $=2.3 \mathrm{scfm}$ to the deep annular spargers $+0.5 \mathrm{scfm}$ to the shallow central spargers

- Idle Operation-1.32 scfm total sparge air $=1.06 \mathrm{scfm}$ to the deep annular spargers $+0.26 \mathrm{scfm}$ to the shallow central spargers (or on a per tube basis, $1.32 \mathrm{scfm}$ divided by the total number of PEP spargers $=0.22 \mathrm{scfm} /$ tube for six PEP spargers)

Except for idle operation, these sparge rates were determined by matching the calculated WTP design basis sparge gas superficial velocity at specified elevations: $1.09 \mathrm{~cm} / \mathrm{s}$ at $2 / 3$ of the elevation above the deep sparger nozzles; and $0.61 \mathrm{~cm} / \mathrm{s}$ at $1 / 2$ of the elevation (=1/2 depth) of the shallow spargers (which equals 0.85 of the deep sparger elevation for a vessel fill level to tank diameter ratio of $1.8 H_{s l} / D$ ). As noted in the calculations above, higher or lower total sparge rates can be specified to produce reasonably less or more conservative "mixing intensity" in the PEP UFP-2 vessel. For example, a total sparge air flow rate of $11.8 \mathrm{scfm}$ (instead of $14.8 \mathrm{scfm}$ ) could be used at $25^{\circ} \mathrm{C}$; it is based on matching the WTP design basis superficial velocity at sparger nozzle depth.

${ }^{1}$ Claghorn R and P Waddell. 2007. System Description for Pulse Jet Mixers and Supplemental Mixing Subsystems. 24590-WTP-3YD-50-00003 Rev B, Bechtel National, Inc., Richland, Washington. 
Operationally, the higher sparge rates based on $25^{\circ} \mathrm{C}$ scaling could be used in low-temperature processes, while heating to caustic leaching temperature, and during cool down if enhanced evaporative cooling is required. During heat up (and cool down), a transition temperature (e.g., $90^{\circ} \mathrm{C}$ ) can be specified where the sparge flow rate is switched from the high set point to the lower flow rate based on nominal leaching conditions (e.g., $100^{\circ} \mathrm{C}, 5.0-\mathrm{M} \mathrm{NaOH}$ slurry). An alternative to this bimodal approach is to define condition-dependent flow rates. Equation (B.9), or more generally Equations (B.5) and (B.6), can be applied to determine the sparge air flow rates required to produce specified $U^{*}$ values for any given slurry density, fill-level, temperature, and saturation partial pressure. Likewise, these equations [and Equation (B.10)] can be used to calculate an expected sparge gas superficial velocity for given sparge air flow rates and slurry properties.

\section{B.1.3.3 Layout of Spargers in the PEP UFP-2 Vessel}

The previous section provided guidance on PEP-scale UFP-2 sparge air flow rates for low-temperature operations and for higher temperature processes such as caustic leaching. In this section, the number and layout of spargers in the PEP vessel is addressed. In this example, the recommended nominal sparge air flow rates for $25^{\circ} \mathrm{C}$ operation, 14.8 -scfm total in the PEP vessel and 360-scfm total in the WTP vessel, are used as calculation bases. The general approach and specific calculations are discussed in Section B.2.

The full-scale WTP vessel uses 16 spargers: six shallow central spargers above the PJM cluster; five deep "type 1" annular spargers at 67-in. radius; and five deep "type 2" annular spargers at 75-in. radius. Table B. 1 summarizes the split of the total $360 \mathrm{scfm}$ WTP design basis flow rate amongst these spargers and the corresponding calculated ROB and ZOI diameters. As noted previously, the WTP vessel spargers are distributed such that the nozzles are separated by $2 / 3$ of the calculated ZOI diameter, $D_{Z O I}$. In this way, ZOI circles of neighboring spargers just meet adjacent ROB circles, as projected on the slurry surface, and the upward flow of bubbles and slurry in ROBs interferes minimally with the downward recirculation in ZOIs.

Table B.1. ROB and ZOI Diameters for the PEP UFP-2 Vessel for Varying Numbers of Spargers Compared to the WTP Full-Scale Design Basis

\begin{tabular}{|c|c|c|c|c|c|c|c|}
\hline \multirow[b]{2}{*}{ Case } & \multirow{2}{*}{$\begin{array}{c}\text { Sparger } \\
\text { Location, Type }\end{array}$} & \multirow{2}{*}{$\begin{array}{c}\text { No. of } \\
\text { Spargers }\end{array}$} & \multicolumn{2}{|c|}{ Sparge Rate (scfm) } & \multicolumn{3}{|c|}{ Diameter (in) } \\
\hline & & & Per Tube & Total & ROB & $\mathrm{ZOI}$ & $2 / 3 \mathrm{ZOI}$ \\
\hline \multirow{4}{*}{$\begin{array}{l}5^{\circ} \mathrm{C} \text { WTP Scale; Design } \\
\text { Basis }\end{array}$} & Deep, type 1 & 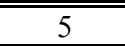 & 39 & 194 & 31 & 94 & 63 \\
\hline & Deep, type 2 & 5 & 20 & 100 & 24 & 75 & 50 \\
\hline & Shallow & 6 & 11 & 66 & 23 & 71 & 47 \\
\hline & All & 16 & N/A & 360 & $\mathrm{~N} / \mathrm{A}$ & $\mathrm{N} / \mathrm{A}$ & $\mathrm{N} / \mathrm{A}$ \\
\hline \multirow{10}{*}{$\begin{array}{l}25^{\circ} \mathrm{C} \text { PEP Scale; } \\
U^{*} \text { matched at reference } \\
\text { elevations: } 0.67 \text { of deep } \\
\text { sparger elev. \& } 0.5 \text { of } \\
\text { shallow sparger elev. }\end{array}$} & Deep, type 1 & 5 & 1.6 & 7.8 & 12 & 37 & 25 \\
\hline & Deep, type 2 & 5 & 0.8 & 4.0 & 10 & 30 & 20 \\
\hline & Shallow & 6 & 0.5 & 3.0 & 8 & 26 & 17 \\
\hline & All & 16 & $\mathrm{~N} / \mathrm{A}$ & 14.8 & N/A & $\mathrm{N} / \mathrm{A}$ & $\mathrm{N} / \mathrm{A}$ \\
\hline & Deep & 5 & 2.4 & 11.9 & 14 & 43 & 29 \\
\hline & Shallow & 1 & 3.0 & 3.0 & 16 & 48 & 32 \\
\hline & All & 6 & N/A & 14.8 & $\mathrm{~N} / \mathrm{A}$ & $\mathrm{N} / \mathrm{A}$ & $\mathrm{N} / \mathrm{A}$ \\
\hline & Deep & 3 & 4.0 & 11.9 & 16 & 51 & 34 \\
\hline & Shallow & 1 & 3.0 & 3.0 & 16 & 48 & 32 \\
\hline & All & 4 & N/A & 14.8 & $\mathrm{~N} / \mathrm{A}$ & N/A & N/A \\
\hline
\end{tabular}

1 Claghorn R and P Waddell. 2007. System Description for Pulse Jet Mixers and Supplemental Mixing Subsystems. 24590-WTP-3YD-50-00003 Rev B, Bechtel National, Inc., Richland, Washington. 
An equivalent analysis was completed for the PEP vessel using all 16 spargers, and the results are shown in Table B.1. The PEP vessel is approximately 37 -in. inside diameter ( $=14.0 \mathrm{ft} / 4.5 \mathrm{scale}$ factor), with a corresponding circumference of $\sim 117$ in. The ten deep spargers, which are distributed uniformly in an angular sense ( $36^{\circ}$ between each), have overlapping calculated ROB circles ( $D_{R O B}$ of 10 to 12 in.) when laid out on the PEP-scale deep sparger nozzle circles (type 1: 14.9-in. radius and 94-in. circumference; and type 2: 16.7-in. radius and 105-in. circumference). Clearly, the still-larger calculated ZOI circles in the PEP overlap more than prescribed as the basis for sparger layout in the WTP vessel.

As noted in the scaling-analysis above (Section B.1.2), the number of spargers required to provide proportional ROB and ZOI area coverage to the WTP-scale system is expected to decrease in the PEP UFP-2 vessel. Results are tabulated in Table B.1 for six total (five deep + one shallow) and four total (three deep + one shallow) spargers in the PEP vessel. The calculated ROB diameter of a single shallow sparger is essentially equal to the diameter of the circle on which the PJMs are located ( 16 in.), at the boundary of the area covered by deep spargers. Therefore, multiple shallow spargers should not be required; a single sparger centered over the PJM cluster is sufficient. Based on the calculated ROB and ZOI diameters, the results suggest that four spargers may be adequate, although the symmetry provided by the five deep spargers in the six sparger system is preferred. In addition to symmetry, another practical consideration is making use of the (scaled) radial and angular sparger locations in the PEP. The five deep PEP spargers would most logically be placed in the "type 1" positions, which are set in between adjacent PJMs ( $\sim 15$-in. radius, $72^{\circ}$ angular distribution).

If the more conservative $25^{\circ} \mathrm{C}$ PEP vessel sparge air flow rate of $11.8 \mathrm{scfm}$ (based on matching the WTP design basis superficial velocity at nozzle depth) is used in the analysis, similar conclusions are drawn regarding the recommended number and locations of spargers.

\section{B.2 Steam Ring Purge Air Scaling}

The replacement of single-point steam injectors with multiple-orifice steam rings in the UFP-1 and UFP-2 vessels introduces another source of sparge air. While a key purpose of the air flow to the steam rings in the WTP UFP vessels is to prevent the back-flow of slurry into the rings when steam is not flowing, ${ }^{1}$ the mixing energy contributions are a more significant factor for establishing purge air flow rates in the PEP UFP vessels. An optimal PEP steam ring system design would address both the backflow prevention and mixing criteria. However, as noted below, the as-built PEP vessel steam rings preclude proper scaling of the purge air flow rates to simultaneously satisfy both requirements. Since back-flow prevention is a lesser factor in the more readily accessible PEP system processing nonradioactive simulant slurries, and so as not to "overmix" the PEP UFP vessels, purge air flow rates are recommended to produce comparable mixing intensity.

\section{B.2.1 Purge Air Nozzle Velocity}

Preventing back-flow of slurry into the orifices of the steam ring using purge air is a function of the air velocity through the orifices (i.e., nozzles). Assuming the steam rings are placed horizontally in the

\footnotetext{
1 The purpose and some operational details of the steam ring purge air systems in the WTP UFP vessels are described in the draft document "Steam Sparger Operation - 050708 draft.doc" attached in email from Bill Peiffer (Bechtel National, Inc., Richland, Washington) on 5/7/08 and 6/5/08. Record copies are included in PNNL CCPWTPSP-456.
} 
slurry at a fixed elevation within the UFP vessels and the hydrostatic pressure is equal at each of the $n_{n o z}$ symmetrically-distributed and uniformly-sized circular orifices, the effective average nozzle velocity $u_{n o z}$ is given by

$$
u_{n o z}=\frac{Q_{t o t}}{n_{n o z} A_{n o z}}=\frac{Q_{t o t}}{n_{n o z} \pi D_{n o z}^{2} / 4}
$$

In this equation, $Q_{t o t}$ is the total actual flow rate of air delivered to the steam ring and flowing through all nozzles, and $A_{n o z}$ is the cross-section of each orifice, which is also represented in terms of its diameter $D_{n o z}$.

Here, it is assumed that purge air velocity at the nozzles is the primary consideration in preventing back-flow of slurry into the steam rings. To maintain a constant nozzle velocity in the WTP and PEP UFP vessel steam ring systems, the combination of number of nozzles, nozzle diameter, and actual air flow rate at the steam ring depth must satisfy Equation (B.21). Preliminary WTP UFP vessel steam ring design information suggests that a minimum target velocity of $70 \mathrm{ft} / \mathrm{s}$ through more than one hundred 1/8-in. diameter orifices will be required. ${ }^{1}$ The number and size (also 1/8-in. diameter) of the PEP UFP vessel steam ring orifices has been defined in as-built drawings, ${ }^{2}$ leaving air flow rate as the only design variable to achieve the target velocity. Since the WTP and PEP steam ring purge orifices have the same cross-section, the PEP and WTP vessel purge air flow rates are related as follows at constant nozzle velocity

$$
Q_{\text {tot }, P E P}=Q_{\text {tot }, W T P} \frac{n_{n o z, P E P}}{n_{n o z, W T P}}
$$

Substituting the preliminary (WTP) and as-built (PEP) steam ring system design information ${ }^{1,2}$ into Equation (B.22) results in estimated PEP vessel air flow rates of $24.3 \mathrm{acfm}$ in UFP-1 and $13.2 \mathrm{acfm}$ in UFP-2 to achieve a nozzle velocity of $70 \mathrm{ft} / \mathrm{s}^{3}$ As shown in the following section, these flow rates are about six- to eight-times higher than those needed to achieve comparable purge-air induced bubble mixing intensity in the WTP and PEP UFP vessels.

${ }^{1}$ Operational details of the steam ring purge air systems in the WTP UFP vessels are described in the draft document "Steam Sparger Operation - 050708 draft.doc" attached in email from Bill Peiffer (Bechtel National, Inc., Richland, Washington) on 5/7/08 and 6/5/08, and in an email from Douglas Vo (Bechtel National, Inc., Richland, Washington) on 3/19/08. The latter specifies the number of $1 / 8$-in. diameter orifices (150 in UFP-1 and 120 in UFP-2) as well as the nominal actual air flow rates (53.662 acfm in UFP-1 and $42.930 \mathrm{acfm}$ in UFP-2) necessary to achieve a $70 \mathrm{ft} / \mathrm{s}$ target nozzle velocity [which was confirmed using Equation (B.21)]. Record copies of the emails and attachment are included in PNNL CCP-WTPSP-456.

2 The Tessenderlo Kerley Services as-built drawing P4-T01A-Sparg-SHT 3, Rev 0 for the PEP UFP-1 prototype vessel (UFP-VSL-T01A) shows sixty eight (68) 1/8-in. diameter orifices in the steam ring, and the as-built drawing P5-T02A-Sparg-SHT 2, Rev 0 for the PEP UFP-2 prototype vessel (UFP-VSL-T02A) shows forty (40) 1/8-in. diameter orifices in the steam ring.

${ }^{3}$ In UFP-1, $Q_{t o t, P E P}=24.3 \mathrm{acfm}=53.662 \mathrm{acfm} *(68 / 150)$; and in UFP-2, $Q_{t o t, P E P}=13.2 \mathrm{acfm}=42.930 \mathrm{acfm} *$ $(40 / 120)$ 


\section{B.2.2 Purge Air Mixing Intensity}

The scaling of steam ring purge air flow rates in the PEP UFP vessels to match the expected mixing energy produced by the corresponding WTP systems is analogous to that shown above for the dedicated air spargers in UFP-2. In each of the WTP and PEP UFP-1 and UFP-2 vessels, the steam rings are located in the annular space between the outside of the PJMs and the inner vessel wall surface at an elevation above the lower vessel tangent line where the 2:1 semi-elliptical bottom head transitions to a vertical cylinder. ${ }^{1}$ Consistent with the analysis of the "deep" UFP-2 spargers in Section B.1.3, the steam ring purge air is assumed to provide mixing in the annular volume of slurry between the nozzle depth and the slurry surface in each of the UFP vessels. In Section B.1.3.1, the annular area affected by the deep spargers is estimated as $68.3 \%$ of the UFP-2 vessel cross-section, and this same area is assumed to be impacted by steam ring purge air. Likewise in UFP-1, the annular area is estimated to be $56.7 \%$ of the vessel cross-section. ${ }^{2}$

Like the earlier analysis of dedicated UFP-2 spargers, steam ring purge air flow rate scaling in each of the UFP vessels is considered for a set of bounding and nominal conditions. In all cases, the PEP vessel air flow rates are based on matching the superficial gas velocity $U^{*}$ to those estimated for comparable conditions in the WTP vessels:

- $25^{\circ} \mathrm{C}$ operation - neglecting evaporation of water into sparge air bubbles and assuming no change in air temperature:

- Matching $\mathrm{U}^{*}$ at Nozzle Depth

- Matching $U^{*}$ at Intermediate Depth - selecting a reference elevation at which both the local superficial velocity [Equation (B.5)] and the integrated bubble power per unit volume of affected slurry $\left[\mathrm{P}_{\mathrm{bV}}\right.$, Equation (B.4)] are nominally the same in the WTP and PEP vessels

- Matching $U^{*}$ at the Slurry Surface

\footnotetext{
${ }^{1}$ The location of the steam rings in the WTP UFP vessels is noted to be $3 \mathrm{ft}$ above the vessel tangent line and 6 in inside of the vessel shell as described in the draft document "Steam Sparger Operation - 050708 draft.doc" [attached in email from Bill Peiffer (Bechtel National, Inc., Richland, Washington) on 5/7/08 and 6/5/08; record copies are included in PNNL CCP-WTPSP-456.]. Per the Tessenderlo Kerley Services as-built drawing P4-T01A-Sparg-SHT 2, Rev 0 for PEP UFP-1, a 50-in. diameter steam ring in the 54-in. inside diameter vessel is 10.75 in. above the lower tangent line; and per the as-built drawing P5-T02A-Sparg-SHT 2, Rev 0 for PEP UFP-2, a 26.875-in. diameter steam ring in the 37.375-in. inside diameter vessel is 8 in. above the lower tangent line.

${ }^{2}$ With reference to WTP UFP-1 vessel drawings (e.g., UFP-VSL-00001B Drawing No. 24590-PTF-MV-UFP-00002, Rev. 2, Bechtel National, Inc., Richland, Washington), the "annular" region between the vessel wall and the outside of the eight 36-in. I.D. PJMs (in two groups of four) is roughly given by a "square in a circle". Considering the four PJMs with centers located at a radius of $6 \mathrm{ft}-8 \mathrm{in}$. and separated angularly by $90^{\circ}$, the vertices of the "square" measured on diagonals from the center of the tank through the center of the PJMs to the outside edges of the PJMs (1-in. wall thickness assumed) are $8 \mathrm{ft}-3$ in. (= $6 \mathrm{ft}-8 \mathrm{in} .+36 \mathrm{in} . / 2+1 \mathrm{in}$.). The area of a square defined by halfdiagonals of length $8.25 \mathrm{ft}$ is $136 \mathrm{ft}^{2}\left[=4 * 0.5^{*}(8.25 \mathrm{ft})^{2}\right]$. By difference, the annular region outside the square is estimated to be $\sim 178 \mathrm{ft}^{2}$ of the vessel cross-section $\left[314 \mathrm{ft}^{2}=\pi^{*}(20 \mathrm{ft})^{2} / 4\right]$. The perimeter of the square drawn on a sectional view of the vessel cuts through the outer portions of each of the eight PJMs; this included PJM area, which clearly is impervious to sparger activity, is compensated by open area between the PJMs where the sparger influence could extend inward (in the square). The approximate area fraction of steam ring purge air influence is 0.567 $\left(=178 \mathrm{ft}^{2} / 314 \mathrm{ft}^{2}\right)$, and because of linear geometric scaling of the vessels, it is assumed to apply to the PEP UFP-1 vessel as well.
} 
- $100^{\circ} \mathrm{C}$ operation - considering expansion of sparge air bubbles due to temperature changes and evaporation of water from a slurry of nominal composition (e.g., 5-M NaOH solution) into the bubbles:

- Matching $U^{*}$ at Intermediate Depth - using the reference elevation established to match $U^{*}$ and $P_{b V}$ for $25^{\circ} \mathrm{C}$ operations

As with the UFP-2 sparger analysis of Section B.1.3.2, matching $U^{*}$ at intermediate depth is the basis for the final recommended PEP flow rates. Other matching criteria are assessed for completeness and to provide upper bounds on the PEP vessel flow rates.

Unlike the analysis of dedicated spargers in UFP-2, the WTP vessel steam ring purge air flow rates are not expected to be adjusted as a function of slurry temperature. This is so because the primary purpose of the purge air flow is preventing back-flow of slurry into the steam rings, not mixing the slurry in the vessel. Therefore, in the purge air flow rate scaling analysis, only the PEP vessel flow rates are adjusted to match the calculated $U^{*}$ of the corresponding WTP UFP vessel for the specified operating conditions. The WTP vessel purge air flow rates are the proposed nominal values in standard units, $87.687 \mathrm{scfm}$ in UFP-1 and $70.15 \mathrm{scfm}$ in UFP-2. ${ }^{1}$

In the sections below, steam ring purge air flow rate scaling analysis details are outlined for each vessel type. The resulting recommendations for PEP vessel flow rates are summarized in a separate section. The following simplifying assumptions are applied to both the UFP-1 and UFP-2 purge air scaling calculations:

- Standard state - The "standard" gas reference state is assumed to be $25^{\circ} \mathrm{C}$ and $1.0 \mathrm{~atm}$; this applies to flow rates given in scfm (standard cubic feet per minute).

- Ambient pressure - For the purpose of these calculations, the ambient pressure in the vessel headspace above the slurry, $P_{a}$, is assumed to be $1.0 \mathrm{~atm}(760 \mathrm{~mm} \mathrm{Hg})$.

- Effective saturation partial pressure - In $25^{\circ} \mathrm{C}$ operation calculations, the air bubbles are assumed to be dry such that the effective saturation partial pressure $\left(\eta P_{\text {sat }}\right)$ is 0 . At $100^{\circ} \mathrm{C}, P_{\text {sat }}$ is assumed to be that for a slurry composition equivalent to 5-M sodium hydroxide solution, and the saturation fraction $\eta$ in the bubbles is assumed to be 1 , giving an effective saturation partial pressure of $616 \mathrm{~mm} \mathrm{Hg}(0.81 \mathrm{~atm})$.

\section{B.2.2.1 Purge-Gas Flow Rate and Mixing Intensity in the PEP UFP-1 Vessel}

To relate the standard volumetric flow rate of purge air to the actual flow rate of gas (air and water vapor) at any elevation between the steam ring and the slurry surface [Equation (B.10)], or inversely, to

\footnotetext{
1 "Preliminary" WTP UFP vessel steam ring system operational parameters, including nominal standard and actual air flow rates, are provided in an email from Douglas Vo (Bechtel National, Inc., Richland, Washington) on 3/19/08. Here, the standard flow rates in the email are used as "proposed values" and actual flow rates as a function of depth in the slurry, slurry physical properties, and other assumed operating conditions are calculated. A record copy of the email is included in PNNL CCP-WTPSP-456.
} 
determine the standard flow rate required to achieve a specified superficial velocity [Equation (B.9)], the following system information is required:

- Total slurry depth - In the WTP UFP-1 vessel, the nominal batch height is given in a vessel sizing calculation as $24.88 \mathrm{ft}(7.58 \mathrm{~m}) .{ }^{1}$ This results in a fill height to vessel diameter aspect ratio of 1.24 $\left(=H_{s l} / D=24.88 \mathrm{ft} / 20.0 \mathrm{ft}\right) .{ }^{2}$ Using a linear geometric scale factor of 4.5 to estimate the PEP vessel diameter (53.33 in., compared to an as-built value of 54 in.) and applying the $H_{s l} / D$ factor gives a PEP UFP-1 total slurry depth of $5.53 \mathrm{ft}(1.69 \mathrm{~m})$. The same result is obtained directly by dividing the WTP vessel total slurry depth by the scale factor.

- Steam ring depth - In the WTP UFP-1 vessel, the steam ring is assumed to be located $3 \mathrm{ft}$ above the lower vessel tangent line, ${ }^{3}$ which corresponds to $8 \mathrm{ft}(2.44 \mathrm{~m})$ above tank bottom center $(=3 \mathrm{ft}+$ $20 \mathrm{ft} / 4$, where the latter term is the inside height of a 2:1 semi-elliptical head in a 20 -ft diameter vessel). The steam ring depth, $H_{s r}$, is the total slurry depth minus the steam ring elevation from the tank bottom, $16.88 \mathrm{ft}(=24.88 \mathrm{ft}-8 \mathrm{ft} ; 5.15 \mathrm{~m})$. Using a scale factor of 4.5 , the PEP UFP-1 steam ring depth is $3.75 \mathrm{ft}(1.14 \mathrm{~m}){ }^{4}$

- Effective sparge area - As noted above, the effective sparge area for the steam rings in UFP-1 is an annular region covering $56.7 \%$ of the vessel cross section $\left[178 \mathrm{ft}^{2}\right.$ in the WTP vessel and $8.79 \mathrm{ft}^{2}$ $\left(=178 \mathrm{ft}^{2} /(4.5)^{2}\right)$ in the PEP vessel].

- Slurry density - The slurry in the WTP and PEP UFP-1 vessels is assumed to have a nominal density of $1.25 \mathrm{~kg} / \mathrm{L} .^{5}$ This is less than in UFP-2 $(1.35 \mathrm{~kg} / \mathrm{L}$ assumed $)$ as the solids content is expected to be lower in UFP-1.

- WTP purge air flow rate - As noted above, the proposed nominal WTP UFP-1 vessel steam ring purge air flow rate is $87.7 \mathrm{scfm}$.

Using this information and equations in Section B.1.1, the superficial velocity of the WTP vessel at various elevations $\left[U^{*}(z)\right]$ and operating conditions are evaluated. Subsequently, the PEP vessel standard air flow rate required to match the superficial velocity of the WTP vessel is determined. The UFP-1 vessel results for the four cases identified in the introduction of Section B.2.2 are as follows:

- $25^{\circ} \mathrm{C}$ Operation, Matching $U^{*}$ at the Slurry Surface - Neglecting bubble expansion due to water vapor at low temperature, the gas flow rate leaving the slurry surface is equal to the standard feed

${ }^{1}$ Sheet 15 of Bauer, JM. 2008. Vessel Sizing Calculations for Ultrafiltration Feed Preparation Vessels UFP-VSL00001A/B. 24590-PTF-M6C-UFP-00004 Rev. D, Bechtel National, Inc., Richland, Washington.

2 The vessel inner shell diameter of $20 \mathrm{ft}$ is taken from UFP-VSL-00001B Drawing No. 24590-PTF-MV-UFP-00002, Rev. 2, Bechtel National, Inc., Richland, Washington.

3 The steam rings in the WTP UFP vessels are noted to be $3 \mathrm{ft}$ above the bottom vessel tangent line in the document "Steam Sparger Operation - 050708 draft.doc" [attached in email from Bill Peiffer (Bechtel National, Inc., Richland, Washington) on 5/7/08 and 6/5/08]. Record copies are included in PNNL CCP-WTPSP-456.

${ }^{4}$ Per the as-built drawing P4-T01 A-Sparg-SHT 2, Rev 0 for PEP UFP-1, the steam ring is 10.75 in. above the lower vessel tangent line. Based on a geometric scale factor of 4.5, the PEP steam rings should be located only 8 in. above the tangent line (= 36 in the WTP vessel/4.5). In PEP UFP-1 the steam ring is probably located slightly higher because of interference with other existing piping (shown in the drawing). The $0.23 \mathrm{ft}$ (2.75 in.) difference in scaled and as-built PEP nozzle depths has a negligible impact on the calculated purge air flow rates $(<0.1 \mathrm{scfm})$.

${ }^{5}$ For example, sheets 13 and 14 of a UFP-1 cooling jacket design calculation specify a slurry specific gravity ranging from 1.188 to 1.268 for various vessel operating modes: Kenworthy, L. 2004. Design of a Cooling Jacket for Ultrafiltration Feed Preparation Vessels. 24590-PTF-MVC-UFP-00007 Rev. B, Bechtel National, Inc., Richland, Washington. 
flow rate and $U^{*}$ is $0.25 \mathrm{~cm} / \mathrm{s}$. The corresponding PEP air purge rate, $4.3 \mathrm{scfm}$, is calculated by simple area scaling of the WTP vessel standard purge air flow rate using a scale factor $S$ of 4.5 [i.e., $\left.4.3 \mathrm{scfm}=87.7 \mathrm{scfm} /(4.5)^{2}\right]$.

- $25^{\circ} \mathrm{C}$ Operation, Matching $U^{*}$ at Nozzle Depth - Neglecting bubble expansion due to water vapor at low temperature, the calculated gas flow rate at nozzle depth in the WTP vessel is $54.0 \mathrm{acfm}$ and $U^{*}$ is $0.15 \mathrm{~cm} / \mathrm{s}$. The corresponding PEP air purge rate is $3.0 \mathrm{scfm}$.

- $25^{\circ} \mathrm{C}$ Operation, Matching $U^{*}$ at Intermediate Depth - Neglecting bubble expansion due to water vapor at low temperature, the calculated gas flow rate at a reference elevation of $z / H_{s r}$ of 0.60 (i.e., $60 \%$ of the steam ring depth above the nozzles or $40 \%$ of the steam ring depth below the surface) in the WTP vessel is $70.2 \mathrm{acfm}$ and $U^{*}$ is $0.20 \mathrm{~cm} / \mathrm{s}$. The corresponding PEP air purge rate is $3.7 \mathrm{scfm}$.

- $100^{\circ} \mathrm{C}$ Operation, Matching $U^{*}$ at Intermediate Depth - Considering air bubble expansion due to thermal effects and due to the inclusion of water vapor in equilibrium with the slurry composition, the calculated $U^{*}$ is $0.71 \mathrm{~cm} / \mathrm{s}$ at a reference elevation $z / H_{s r}$ of 0.60 for a gas flow rate of $87.7 \mathrm{scfm}$ in the WTP vessel. The corresponding PEP air purge rate is $2.4 \mathrm{scfm}$.

\section{B.2.2.2 Purge-Gas Flow Rate and Mixing Intensity in the PEP UFP-2 Vessel}

Much of the information needed to estimate the scaled PEP UFP-2 vessel steam ring purge air flow rates is identical to that used in the analysis of dedicated sparger flow rates (Sections B.1.3.1 and B.1.3.2). This information and other parameters specific to the UFP-2 steam ring system are summarized below for convenience:

- Total slurry depth - The WTP UFP-2 vessel batch height for a nominal slurry fill level of $1.8 H_{s l} / D$ is $25.2 \mathrm{ft}(7.68 \mathrm{~m})$. Using a linear geometric scaling factor of 4.5 gives a PEP UFP-2 total slurry depth of $5.60 \mathrm{ft}(1.71 \mathrm{~m})$.

- Steam ring depth - In the WTP UFP-2 vessel, the steam ring is assumed to be located $3 \mathrm{ft}$ above the lower vessel tangent line, ${ }^{1}$ which corresponds to $6.5 \mathrm{ft}(1.98 \mathrm{~m})$ above tank bottom center $(=3 \mathrm{ft}+$ $14 \mathrm{ft} / 4$, where the latter term is the inside height of a $2: 1$ semi-elliptical head in a $14-\mathrm{ft}$ diameter vessel). The steam ring depth, $H_{s r}$, is the total slurry depth minus the steam ring elevation from the tank bottom, $18.7 \mathrm{ft}(=25.2 \mathrm{ft}-6.5 \mathrm{ft} ; 5.70 \mathrm{~m})$. Using a scale factor of 4.5, the PEP UFP-2 steam ring depth is $4.16 \mathrm{ft}(1.27 \mathrm{~m}){ }^{2}$

- Effective sparge area - As noted above, the effective sparge area for the steam rings in UFP-2 is an annular region covering $68.3 \%$ of the vessel cross section $\left[105 \mathrm{ft}^{2}\right.$ in the WTP vessel and $5.2 \mathrm{ft}^{2}(=$ $\left.105 \mathrm{ft}^{2} /(4.5)^{2}\right)$ in the PEP vessel].

- Slurry density - The slurry in the WTP and PEP UFP-2 vessels is assumed to have a density of $1.35 \mathrm{~kg} / \mathrm{L}$.

- WTP purge air flow rate - As noted above, the proposed nominal WTP UFP-2 vessel steam ring purge air flow rate is $70.15 \mathrm{scfm}$.

\footnotetext{
${ }^{1}$ The steam rings in the WTP UFP vessels are noted to be $3 \mathrm{ft}$ above the bottom vessel tangent line in the document "Steam Sparger Operation - 050708 draft.doc" [attached in email from Bill Peiffer (Bechtel National, Inc., Richland, Washington) on 5/7/08 and 6/5/08]. Record copies are included in PNNL CCP-WTPSP-456.

2 Per the as-built drawing P4-T02A-Sparg-SHT 2, Rev 0 for PEP UFP-2, the steam ring is 8 in. above the lower vessel tangent line, consistent with a geometric scale factor of 4.5 (i.e., 8 in. $=36$ in the WTP vessel/4.5).
} 
Using this information and equations in Section B.1.1, the superficial velocity of the WTP vessel at various elevations $\left[U^{*}(z)\right]$ and operating conditions are evaluated. Subsequently, the PEP vessel standard air flow rate required to match the superficial velocity of the WTP vessel is determined. The UFP-2 vessel results for the cases of interest are as follows:

- $25^{\circ} \mathrm{C}$ Operation, Matching $U^{*}$ at the Slurry Surface - Neglecting bubble expansion due to water vapor at low temperature, the gas flow rate leaving the slurry surface is equal to the standard feed flow rate and $U^{*}$ is $0.34 \mathrm{~cm} / \mathrm{s}$. The corresponding PEP air purge rate, $3.5 \mathrm{scfm}$, is calculated by simple area scaling of the WTP vessel standard purge air flow rate using a scale factor $S$ of 4.5 [i.e., $\left.3.5 \mathrm{scfm}=70.2 \mathrm{scfm} /(4.5)^{2}\right]$.

- $25^{\circ} \mathrm{C}$ Operation, Matching $U^{*}$ at Nozzle Depth - Neglecting bubble expansion due to water vapor at low temperature, the calculated gas flow rate at nozzle depth in the WTP vessel is $40.2 \mathrm{acfm}$ and $U^{*}$ is $0.19 \mathrm{~cm} / \mathrm{s}$. The corresponding PEP air purge rate is $2.3 \mathrm{scfm}$.

- $25^{\circ} \mathrm{C}$ Operation, Matching $U^{*}$ at Intermediate Depth - Neglecting bubble expansion due to water vapor at low temperature, the calculated gas flow rate at a reference elevation of $z / H_{s r}$ of 0.60 in the WTP vessel is $54.0 \mathrm{acfm}$ and $U^{*}$ is $0.26 \mathrm{~cm} / \mathrm{s}$. The corresponding PEP air purge rate is $2.8 \mathrm{scfm}$.

- $100^{\circ} \mathrm{C}$ Operation, Matching $U^{*}$ at Intermediate Depth - Considering air bubble expansion due to thermal effects and due to the inclusion of water vapor in equilibrium with the slurry composition, the calculated $U^{*}$ is $0.87 \mathrm{~cm} / \mathrm{s}$ at a reference elevation $z / H_{s r}$ of 0.60 for a gas flow rate of $70.2 \mathrm{scfm}$ in the WTP vessel. The corresponding PEP air purge rate is $1.8 \mathrm{scfm}$.

\section{B.2.2.3 Summary of Recommended Purge Air Flow Rates in the PEP UFP Vessels}

In the analyses above, the $25^{\circ} \mathrm{C}$ cases in which $U^{*}$ is matched at nozzle depth $\left(z / H_{s r}=0\right)$ and the slurry surface $\left(z / H_{s r}=1\right)$ provide lower and upper bounds for PEP UFP-1 steam ring purge air flow rates for low temperature operation. Matching $U^{*}$ at intermediate depth (e.g., $0.60 z / H_{s r}$ ) is a compromise in which the calculated superficial velocity of the PEP system is greater than the WTP system below the reference elevation and lower above it. On average, the computed bubble mixing power in the PEP and WTP systems is about equal using this scaling approach. In the cases detailed above, note that the superficial velocity, and therefore mixing power, is more than three-times greater at $100^{\circ} \mathrm{C}$ than at $25^{\circ} \mathrm{C}$ (e.g., $0.71 \mathrm{~cm} / \mathrm{s}$ vs. $0.20 \mathrm{~cm} / \mathrm{s}$ in UFP-1; and $0.87 \mathrm{~cm} / \mathrm{s}$ vs. $0.26 \mathrm{~cm} / \mathrm{s}$ in UFP-2). This is due to thermal and water vapor enhancement of the purge air bubble volume at a high temperature and because the WTP vessel steam ring purge rate is assumed to be constant, independent of temperature.

As noted in the two previous sections, the calculated average bubble mixing intensity in WTP and PEP UFP vessels is roughly equal if the purge gas superficial velocity $U^{*}$ is matched at a reference elevation of about $0.6\left(z / H_{s r}\right)$. The calculated PEP vessel purge air flow rates needed to match $U^{*}$ at an intermediate depth are summarized in Table B.2. These are based on proposed WTP vessel purge air flow rates, ${ }^{1}$ which are also reproduced in the table. To match the calculated WTP vessel $U^{*}$ when operating hot (e.g., $\left.100^{\circ} \mathrm{C}\right)$, reduced PEP flow rates are recommended. Following the guidance used for dedicated spargers in UFP-2 in Section B.1.3.2, one could consider using the higher PEP vessel purge air flow rate calculated for $25^{\circ} \mathrm{C}$ operation whenever the slurry temperature in the vessel is $<90^{\circ} \mathrm{C}$ and use the lower air purge rate when the slurry temperature is $\geq 90^{\circ} \mathrm{C}$.

\footnotetext{
1 "Preliminary" WTP UFP vessel steam ring purge air flow rates are provided in an email from Douglas Vo (Bechtel National, Inc., Richland, Washington) on 3/19/08. A record copy is included in PNNL CCP-WTPSP-456.
} 
Table B.2. Recommended Purge Air Flow Rates in the PEP UFP Vessels

\begin{tabular}{|c|c|c|c|c||}
\hline \multirow{2}{*}{$\begin{array}{c}\text { Operating } \\
\text { Temperature }\end{array}$} & \multicolumn{3}{|c|}{ Nominal Steam Ring Air Purge Rate (scfm) } \\
\cline { 2 - 5 } & \multicolumn{2}{|c|}{ UFP-1 } & \multicolumn{2}{c||}{ UFP-2 } \\
\cline { 2 - 5 } & PEP & WTP & PEP & WTP \\
\hline \hline $25^{\circ} \mathrm{C}$ & 3.7 & 87.7 & 2.8 & 70.2 \\
\hline $100^{\circ} \mathrm{C}$ & 2.4 & 87.7 & 1.8 & 70.2 \\
\hline
\end{tabular}

Table B. 2 shows the recommended standard air flow rates. The corresponding calculated actual air flow rates at the steam ring nozzle depth for $25^{\circ} \mathrm{C}$ operation are $3.2 \mathrm{acfm}$ in UFP-1 and $2.4 \mathrm{acfm}$ in UFP-2. Note that the these flow rates are about a factor six- to eight-times less than the flow rate needed to achieve a nozzle velocity of $70 \mathrm{ft} / \mathrm{s}$ (Section B.2.1). Therefore, the expected nozzle velocities in the PEP are reduced by this same factor. These velocities ( 9 to $12 \mathrm{ft} / \mathrm{s}$ ) are still significant and may be sufficient to prevent back-flow of slurry into the steam rings. A lower velocity should be required in the PEP because the steam ring depth and hydrostatic pressure are reduced. 
PNNL-16948

WTP-RPT-160, Rev 0

\section{Distribution}

No. of

Copies

No. of

Copies

\section{ONSITE}

6 Bechtel National, Inc.

V. Guynes (2) H4-02

SM Barnes H4-02

S. Lehrman H4-02

RS Disselkamp H4-02

P. S. Sundar H4-02

12 Pacific Northwest National Laboratory

WL Kuhn

K7-15

BD Hanson

$\mathrm{P} 7-27$

PW Eslinger

K6-52

JL Huckaby

H4-02

DE Kurath

LA Mahoney

K3-52

K7-15

K7-15

P7-22

RA Peterson

K6-28

P8-55

Information Release (2)

K3-52

Project File
No. of

Copies 